\title{
Schwingungsdynamik in O-H... O-verbrückten Aggregaten: FTIR-Spektroskopie vom Nah- bis zum Ferninfraroten
}

\author{
DISSERTATION \\ zur Erlangung des mathematisch-naturwissenschaftlichen Doktorgrades \\ „Doctor rerum naturalium“ \\ der Georg-August-Universität Göttingen \\ im Promotionsprogramm Chemie \\ der Georg-August University School of Science (GAUSS)
}

vorgelegt von

Franz Kollipost

aus Schwerin

Göttingen 2015 


\section{Betreuungsausschuss}

Prof. Dr. Martin A. Suhm,

Institut für Physikalische Chemie, Georg-August-Universität Göttingen Jun.-Prof. Dr. Ricardo A. Mata,

Institut für Physikalische Chemie, Georg-August-Universität Göttingen

\section{Mitglieder der Prüfungskommission}

Referent: Prof. Dr. Martin A. Suhm,

Institut für Physikalische Chemie, Georg-August-Universität Göttingen

Korreferent Jun.-Prof. Dr. Ricardo A. Mata,

Institut für Physikalische Chemie, Georg-August-Universität Göttingen

\section{Weitere Mitglieder der Prüfungskommission}

Prof. Dr. Götz Eckold,

Institut für Physikalische Chemie, Georg-August-Universität Göttingen Prof. Dr. Burkhard Geil,

Institut für Physikalische Chemie, Georg-August-Universität Göttingen

Prof. Jörg Schroeder, Ph.D.,

Institut für Physikalische Chemie, Georg-August-Universität Göttingen

PD Dr. Thomas Zeuch

Institut für Physikalische Chemie, Georg-August-Universität Göttingen

Tag der mündlichen Prüfung: 08.06.2015 
Einzig der Humor, die herrliche Erfindung der in ihrer Berufung zum Größten Gehemmten, der beinahe Tragischen, der höchstbegabten Unglücklichen [...] überzieht und vereinigt alle Bezirke des

Menschenwesens mit den Strahlungen seiner Prismen.

Hermann Hesse, Der Steppenwolf 


\section{Danksagung}

Zum Gelingen dieser Arbeit haben viele Menschen beigetragen, denen ich hier danken möchte. Zuallererst gilt mein herzlicher Dank Prof. Dr. Martin Suhm für das interessante Themengebiet und die intensive Betreuung mit vielen Diskussionen und Anregungen, die trotzdem viel Freiraum für selbstständiges Forschen gelassen hat. Jun.-Prof. Dr. Ricardo Mata danke ich für die Übernahme des Korreferats dieser Arbeit. Besonderer Dank geht auch an Prof. Dr. René Wugt Larsen, der meine Zweitbetreuung im Rahmen des GRK 782 übernommen und wichtige Hilfe bei den Ferninfrarotmessungen geleistet hat. Auch haben die zur Verfügung gestellten Matrixergebnisse aus seiner Arbeitsgruppe grundlegend zum Verständnis des Ferninfrarotbereichs beigetragen.

Mein Dank geht auch an das GRK 782, dass mich während der ersten Hälfte meiner Promotion mit einem Stipendium gefördert und mir viele Konferenzreisen ermöglicht hat. In diesem Sinne danke ich auch der DFG, die das Obertonprojekt finanziell ermöglicht hat. Die schnelle Hilfe der Werkstätten des IPC und des IOBC habe ich oft in Anspruch genommen und möchte mich dafür bei all ihren Mitarbeitern hier bedanken.

Allen aktuellen und ehemaligen Mitarbeitern der Suhmgruppe und der Zeuchgruppe, die mich während meiner Promotion begleitet haben danke ich für die tolle Arbeitsatmosphäre und den Spaß neben der Forschung. Besonderer Dank geht an Jonas Altnöder, Matthias Heger, Robert Medel, Katharina Otto und Anja Poblotzki für das Korrekturlesen einzelner Kapitel. Nicole Borho danke ich für den Aufbau des Filet-Jet und wünsche allen Studenten und Doktorranden, die ihn nach mir nutzen werden, viel Freude bei der Arbeit mit dieser leistungsfähigen Apparatur.

Während meiner Promotion habe ich Daniel Falkowski, Michael Hendrich, Stefan Nehls und Kim Papendorf, der mich bei den Obertonmessungen unterstützt hat, während ihrer Bachelorarbeiten betreut, was mir viel Spaß gemacht hat und für den ich mich hier bei ihnen bedanken möchte.

Hendrik Schröder und Axel Binning haben mich im Zuge eines Abteilungspraktikums bei der Untersuchung von Propandiol unterstützt, wofür ich ihnen sehr dankbar bin. Auch Christoph Dierking, Thorsten Stolper und Robert Medel danke ich für ihren Einsatz während ihrer Praktika und die resultierenden Ergebnisse.

Mein Dank geht auch an meine Kommilitonen, Bandkollegen, Mitbewohner, Freunde und Familienmitglieder, die mich während der Promotion begleitet haben und für Unterstützung, aber vor allem die nötige Zerstreuung abseits der Forschung gesorgt haben. Den wichtigsten Beitrag zum Gelingen der Arbeit außerhalb des fachlichen Rahmens hat Julia Tiemann geleistet, wofür ich ihr in Liebe dankbar bin. 


\section{Inhaltsverzeichnis}

1. Einleitung 1

2. Grundlagen 3

2.1. Modelle für Molekülschwingungen . . . . . . . . . . . . . . . . . . 3

2.2. FTIR-Spektroskopie Wasserstoffbrücken-gebundener Cluster in Überschallexpansionen ......................... 7

3. Experimentelles 11

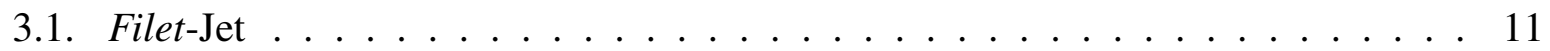

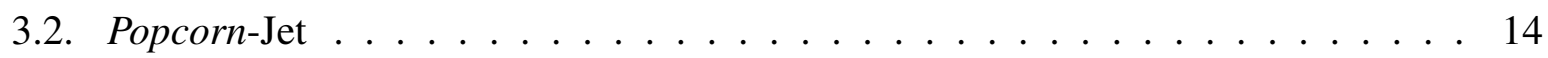

3.3. Detektorenvergleich . . . . . . . . . . . . . . . . . . 14

3.4. Chemikalien . . . . . . . . . . . . . . . . . . 15

3.5. Quantenchemische Rechnungen . . . . . . . . . . . . . . 15

4. O-H Streckschwingungen 17

4.1. Fundamentalübergänge . . . . . . . . . . . . . . . . . . . 17

4.1.1. Messbedingungen . . . . . . . . . . . . . 17

4.1.2. Ethylenglykol . . . . . . . . . . . . . . . . . . . 18

4.1.3. Propylenglykol . . . . . . . . . . . . . . . . . 22

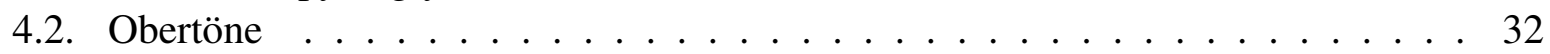

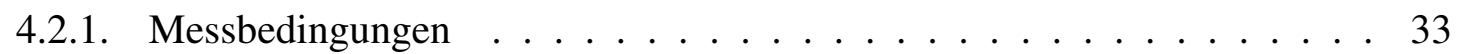

4.2.2. Methanol . . . . . . . . . . . . . . . 36

4.2.3. Ethanol ........................... 41

4.2.4. tert-Butylalkohol . . . . . . . . . . . . . . . . 47

4.2.5. Hexafluorisopropanol . . . . . . . . . . . . . . . . . . . . . 49

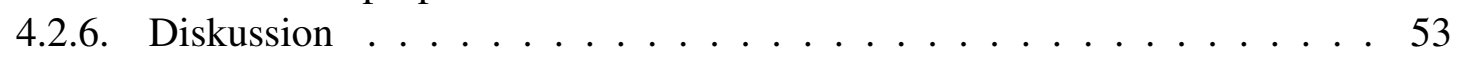

5. O-H Torsionen $\quad 61$

5.1. Messbedingungen . . . . . . . . . . . . . . . . . . 62 62

5.2. Methanol . . . . . . . . . . . . . . . . . . 6 62

5.2.1. Methanol-OH . . . . . . . . . . . . . . . . 62

5.2.2. Methanol-OD . . . . . . . . . . . . . . . . 75

5.2.3. Zusammenfassung .................... 78

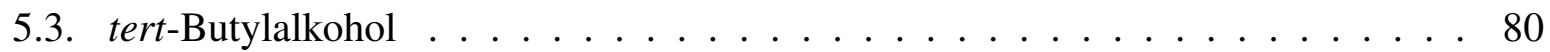

5.3.1. tert-Butylalkohol-OH . . . . . . . . . . . . . . 80 
5.3.2. tert-Butylalkohol-OD . . . . . . . . . . . . . . . . . . 84

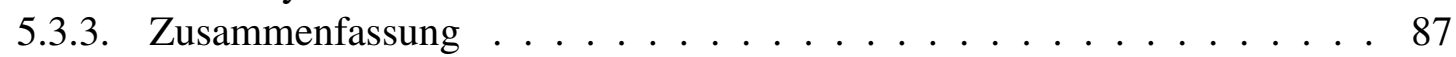

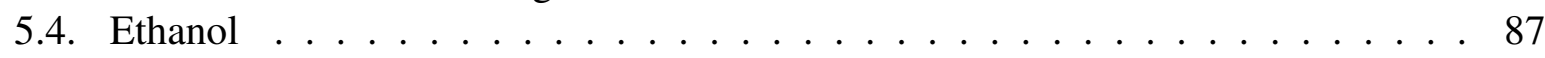

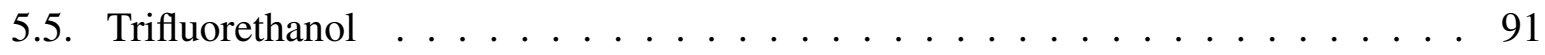

5.5.1. Trifluorethanol-OH . . . . . . . . . . . . . . . . 91

5.5.2. Trifluorethanol-OD . . . . . . . . . . . . . . . . 95

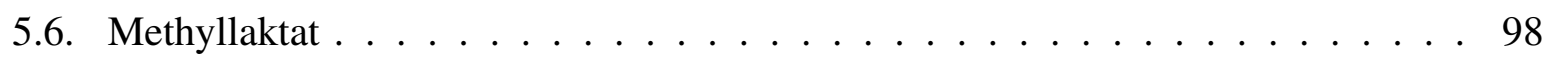

5.7. Diskussion . . . . . . . . . . . . . . . . . 108

6. Ameisensäuredimer 111

6.1. Messbedingungen . . . . . . . . . . . . . . . . . . . 112

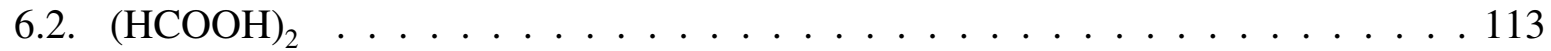

6.3. Deuterierte Isotopologe des Ameisensäuredimers . . . . . . . . . . . . . 123

$\begin{array}{lr}\text { 7. Zusammenfassung \& Ausblick } & 129\end{array}$

A. Anhang i

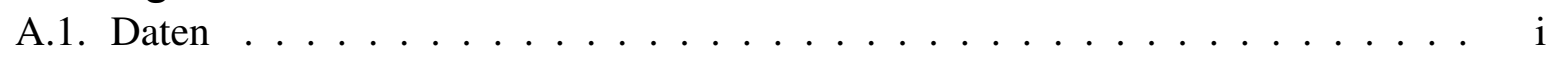

A.2. Ergänzende Spektren . . . . . . . . . . . . . . . . xiv

Literaturverzeichnis $\quad$ xvii

$\begin{array}{ll}\text { Publikationen } & \text { xxxix }\end{array}$ 


\section{Einleitung}

Die Ausbildung von Wasserstoffbrücken bei der Aggregation von Molekülen zu kleinen Clustern bewirkt Veränderungen der Molekülschwingungen. In erster Linie sind dabei die Schwingungen der funktionellen Gruppen betroffen, die unmittelbar an der Ausbildung der Wasserstoffbrücke beteiligt sind.

In dieser Arbeit werden Moleküle mit Hydroxyl-, Carbonyl- und Carboxylgruppen untersucht. Sie bilden in ihren Clustern $\mathrm{O}-\mathrm{H}$... O Wasserstoffbrücken aus. Somit sind besonders die Schwingungen der $\mathrm{O}-\mathrm{H}$ Gruppe für die Charakterisierung dieser Cluster interessant. Die Schwingungsübergänge werden mit Hilfe von FTIR-Spektroskopie der Molekülaggregate detektiert. Dazu werden die Cluster in Überschallexpansionen erzeugt und liegen somit stark gekühlt und isoliert im Vakuum vor. Diese Methodik macht die resultierenden Messergebnisse zu idealen Bezugswerten für quantenchemische Berechnungen kleiner Cluster, die häufig ohne Temperaturund Umgebungseinflüsse durchgeführt werden.

Die O-H Streckschwingung erfährt durch die Ausbildung von Wasserstoffbrücken eine charakteristische Rotverschiebung ihrer Schwingungsfrequenz. ${ }^{1-4}$ Die O-H Streckschwingungen in Wasserstoffbrücken sind verhältnismäßig einfach detektierbar, sodass sie bereits in einer großen Anzahl von Systemen untersucht wurden. ${ }^{5-7}$

Auch im Zuge dieser Arbeit werden in Kapitel 4.1 die Streckschwingungen in Diolen analysiert, um die Strukturen ihrer stabilsten Dimere aufzuklären. Ferner wurden die N-H Streckschwingungen von Pyrrol in gemischten Dimeren und Trimeren mit Aceton und Cyclopentanon untersucht, ${ }^{7}$ um die Charakterisierung des Trimers aus zwei Pyrrolmolekülen und einem Acetonmolekül aus einer früheren $S_{t u d i e}{ }^{8}$ zu überprüfen.

Außerdem wurden die $\mathrm{O}-\mathrm{H}$ Streckschwingungsübergänge von Methanol in gemischten Dimeren, Trimeren und Tetrameren aus Methanol und Aceton gemessen und ihre Strukturen anhand der Absorptionsspektren aufgeklärt. ${ }^{9}$ Dabei sollte untersucht werden, ob sich die energetisch ungünstige Mischung beider Flüssigkeiten auch auf der Ebene ihrer kleinsten Aggregate wiederfinden lässt. Tatsächlich wurde anhand der theoretisch ermittelten Energien der Cluster zwar eine Präferenz für heteromolekulare Dimere und Trimere gefunden. ${ }^{9}$ Im Falle der Tetramere werden aber homomolekulare Aggregate bevorzugt, was als ein Zeichen für die beginnende Entmischung des Systems gewertet werden kann. ${ }^{9}$ Der Grund für die unterschiedlichen energetischen Präferenzen in den kleinen Clustern ist in der Konkurrenz zwischen den intrinsisch stärkeren $\mathrm{O}-\mathrm{H} \cdots \mathrm{O}=\mathrm{C}$ Wechselwirkungen und kooperativen $\mathrm{O}-\mathrm{H} \cdots \mathrm{O}-\mathrm{H}$ Systemen in cyclischen Anordnungen begründet.

Um das theoretische Verständnis für die Rotverschiebungen der $\mathrm{O}-\mathrm{H}$ Streckschwingungen in Wasserstoffbrücken zu verbessern, ist es unerlässlich, über die Änderung der Anharmonizität dieser Schwingung in Kenntnis zu kommen. ${ }^{10}$ Experimentell sind Anharmonizitätskonstanten aus den Frequenzen von Obertönen in Verbindung mit denen von Fundamentalen zugänglich. Dar- 


\section{Einleitung}

um sollen in dieser Arbeit die Obertöne von Alkoholdimeren systematisch im Nahinfrarotbereich untersucht werden. Die Analyse der Systeme ist in Kapitel 4.2 beschrieben und die Ergebnisse wurden bereits publiziert. ${ }^{7,11}$

Neben der Streckschwingung der Hydroxylgruppe wird auch ihre Torsion um die C-O Bindung stark durch die Ausbildung einer Wasserstoffbrücke beeinflusst. Diese innere Rotation der Moleküle wird in ihren Clustern in eine Schwingung umgewandelt, deren Fundamentale im Ferninfrarotbereich gefunden werden kann. Diese Schwingungen führen die O-H Gruppe aus der (nahezu) linearen Anordnung der $\mathrm{O}-\mathrm{H} \cdots \mathrm{O}$ Wasserstoffbrücke heraus und werden auf Grund dieser befreienden Bewegung Librationen genannt. Da sie für $\mathrm{O}-\mathrm{H} \cdots \mathrm{O}$-gebundene Cluster bisher nur wenig untersucht wurden, sollen die existierenden Arbeiten zu den Alkoholtrimeren und -tetrameren ${ }^{12,13}$ hier um Untersuchungen zu ihren Dimeren und deuterierten Clustern erweitert werden. Die Experimente werden in Kapitel 5 beschrieben. Außerdem werden dort die Librationsschwingungen von Methyllaktat untersucht. Die Ergebnisse zur Methanoldimerlibration aus Überschallexpansionen wurden zusammen mit komplementären Spektren des Matrixisolierten Systems publiziert. ${ }^{14}$

Im Ferninfrarotbereich können auch intermolekulare Schwingungen beobachtet werden. Dies sollte hier für das Ameisensäuredimer für die höchstfrequente zwischenmolekulare Schwingung versucht werden. Tatsächlich gelang die Messung dieser Bande, wodurch interessante Rückschlüsse auf die Dissoziationsenergie des Dimers zugelassen wurden. ${ }^{15}$ Außerdem soll hier das Schwingungsspektrum des Ameisensäuredimers und seiner deuterierten Isotopologen im mittleren Infrarotbereich zwischen 600 und $1900 \mathrm{~cm}^{-1}$ charakterisiert werden. Das Vorhaben profitiert von der hohen Konzentration des Dimers in Überschallexpansionen, die die Detektion schwacher Signale vereinfacht, und ist in Kapitel 6 dokumentiert. In Verbindung mit den umfassend charakterisierten Ramandaten ${ }^{16}$ und Untersuchungen im höheren Wellenzahlbereich ${ }^{17}$ sollte sich so ein umfangreiches Bild der Schwingungsübergänge des Ameisensäuredimers ergeben.

Während eines Forschungsaufenthaltes an der MAX-lab Speicherringanlage der Universität Lund in Schweden wurde ein Rotations-aufgelöstes Spektrum von ${ }^{81} \mathrm{BrCH}_{2} \mathrm{~F}$ im Bereich von 920 bis $980 \mathrm{~cm}^{-1}$ gemessen. In Kooperation mit der Universität Venedig konnte es analysiert und veröffentlicht werden. ${ }^{18}$

Der Hauptfokus dieser Arbeit liegt in der Untersuchung der $\mathrm{O}-\mathrm{H}$ Schwingungsdynamik in den Wasserstoffbrücken von Alkoholclustern und insbesondere -dimeren. Dazu ist die Erweiterung des üblicherweise betrachteten Spektralbereichs im mittleren Infraroten notwendig. Die Erschließung schwacher Streckschwingungsobertöne im Nahinfrarotbereich ist dabei ebenso herausfordernd wie die Beobachtung von $\mathrm{O}-\mathrm{H}$ Librationen im Ferninfrarotbereich. Die Verwendung empfindlicher Detektoren und der großen Schlitzdüse des Filet-Jet für die Überschallexpansionen ermöglicht jedoch für diese Arbeit die Detektion der in vielen Fällen äußerst schwachen Signale. 


\section{Grundlagen}

\subsection{Modelle für Molekülschwingungen}

In nichtlinearen Molekülen und Clustern mit $N$ Atomen liegen $3 N-6$ Schwingungsmoden vor. In jeder einzelnen dieser Normalmoden schwingen die Atome des Systems jeweils mit derselben Frequenz und Phase, ${ }^{19}$ ihre gemeinsame Bewegung in einer bestimmten Mode wird von ihrer Normalkoordinate beschrieben. Die Normalmoden in Clustern reichen von Bewegungen nur zweier Atome über Schwingungen innerhalb ganzer Gruppen oder ganzer Moleküle bis hin zur Auslenkung mehrerer Moleküle gegeneinander.

Die Schwingungen der Moleküle resultieren aus der Bewegung der Atomkerne innerhalb der elektronischen Potentialhyperfläche des Systems im Rahmen der Born-Oppenheimer-Näherung. Die Ruhelage der Atomkerne befindet sich an einem Minimum der Potentialhyperfläche. Werden die Atomkerne aus der Ruhelage in eine Richtung ausgelenkt, erfahren sie also stets eine rücktreibende Kraft, die die Schwingung der Kerne hervorruft. Auf Grund der kleinen Dimensionen von Atomen spielen ihre Quanteneigenschaften eine wichtige Rolle. So können nicht beliebige stationäre Schwingungszustände eingenommen werden, sondern nur solche, deren Wellenfunktionen bestimmte Randbedingungen erfüllen. Das führt zu einer Quantelung der möglichen Schwingungsübergänge und führt letztendlich zu den spektroskopisch beobachtbaren scharfen Übergängen zwischen diskreten Schwingungsniveaus. ${ }^{19}$ Außerdem führen die Quanteneigenschaften zur Existenz einer signifikanten Nullpunktsenergie, da die Atomkerne im Sinne der Heisenbergschen Unbestimmtheitsrelation nicht bewegungslos im Potentialminimum verweilen können.

Damit ein Photon unter Anregung einer Schwingungsmode eines Moleküls absorbiert werden kann, muss zum einen seine Energie zu dem Übergang zwischen zwei Schwingungsniveaus passen, zum anderen muss sein elektrisches (oder magnetisches) Feld mit dem Schwingungsübergang wechselwirken können. In Photonen schwingt das elektrische Feld senkrecht zu ihrer Ausbreitungsrichtung. ${ }^{20}$ Dieses Wechselfeld kann mit einem Schwingungsübergang wechselwirken, wenn bei diesem das elektrische Feld des Moleküls ebenfalls schwingt. Dies ist in erster Näherung der Fall, wenn sich bei einem ungeladenen Molekül das Dipolmoment entlang der Schwingungskoordinate ändert.

Je stärker sich das Dipolmoment entlang einer Schwingungskoordinate ändert, desto wahrscheinlicher wird ein Photon absorbiert. Mathematisch ist die Wahrscheinlichkeit des Schwingungsüberganges eines Moleküls proportional zum Quadrat des Übergangsdipolmoments ${ }^{21} \mu_{\mathrm{nm}}^{2}$ (s.a. Formel 5.1 in Kapitel 5.2.1). Dieses kann wiederum theoretisch durch Anwendung des Dipolmomentoperators $\widehat{\mu}$ auf die Schwingungswellenfunktionen $\Psi_{\mathrm{v}^{\prime \prime}}$ und $\Psi_{\mathrm{v}^{\prime}}$ der am Übergang beteiligten Zustände mit den Quantenzahlen $\mathrm{v}^{\prime \prime}$ und $\mathrm{v}^{\prime}$ berechnet werden: 


\section{Grundlagen}

$$
\mu_{\mathrm{v}^{\prime \prime} \mathrm{v}^{\prime}}=\left\langle\Psi_{\mathrm{v}^{\prime \prime}}|\widehat{\mu}| \Psi_{\mathrm{v}^{\prime}}\right\rangle
$$

Der Dipoloperator kann als Taylorreihe um die Ruhelage $r_{\mathrm{e}}$ der Atome entwickelt und auf die Wellenfunktionen angewendet werden, ${ }^{22}$ was hier zur Veranschaulichung nur bis zum Glied 2. Ordnung geschehen soll:

$$
\left\langle\Psi_{\mathrm{v}^{\prime \prime}}|\widehat{\mu}| \Psi_{\mathrm{v}^{\prime}}\right\rangle=\mu_{r_{\mathrm{e}}}\left\langle\Psi_{\mathrm{v}^{\prime \prime}} \mid \Psi_{\mathrm{v}^{\prime}}\right\rangle+\left.\frac{\mathrm{d} \mu}{\mathrm{d} r}\right|_{r_{\mathrm{e}}}\left\langle\Psi_{\mathrm{v}^{\prime \prime}}\left|\left(r-r_{\mathrm{e}}\right)\right| \Psi_{\mathrm{v}^{\prime}}\right\rangle+\left.\frac{1}{2} \frac{\mathrm{d}^{2} \mu}{\mathrm{d} r^{2}}\right|_{r_{\mathrm{e}}}\left\langle\Psi_{\mathrm{v}^{\prime \prime}}\left|\left(r-r_{\mathrm{e}}\right)^{2}\right| \Psi_{\mathrm{v}^{\prime}}\right\rangle \ldots
$$

Da das Integral $\left\langle\Psi_{\mathrm{v}^{\prime \prime}} \mid \Psi_{\mathrm{v}^{\prime}}\right\rangle$ für orthonormierte Wellenfunktionen bei $\mathrm{v}^{\prime \prime} \neq \mathrm{v}^{\prime}$ verschwindet, spielt es für Schwingungsübergänge keine Rolle. Jedoch lässt sich aus dem Glied 0. Ordnung die Auswahlregel für reine Rotationsübergänge $\left(\mathrm{v}^{\prime \prime}=\mathrm{v}^{\prime}\right)$ ableiten, dass die Absorption eines Photons ein permanentes Dipolmoment des Moleküls oder Clusters erfordert.

Das einfachste Modell um Schwingungen von Molekülen und Clustern zu beschreiben, ist die doppelt-harmonische Näherung. ${ }^{20}$ Es wird angenommen, dass die rücktreibende Kraft mit der Auslenkung $\left(r-r_{\mathrm{e}}\right)$ der Atome linear zunimmt. ${ }^{22}$ Diese mechanische Harmonizität ist gleichbedeutend mit einem quadratischen Potential, in dem die Schwingung ausgeführt wird.

Wird die Schrödingergleichung für ein Teilchen in diesem Potential (harmonischer Oszillator) gelöst, so erhält man zum einen Wellenfunktionen die bezüglich $r_{\mathrm{e}}$ symmetrisch oder antisymmetrisch sind und zum anderen äquidistante Energieniveaus. ${ }^{20}$ Für die Schwingungsterme $G(\mathrm{v})$ gilt:

$$
G(\mathrm{v})=\omega_{\mathrm{e}}\left(\mathrm{v}+\frac{1}{2}\right)
$$

Die harmonische Wellenzahl $\omega_{\mathrm{e}}$ entspricht den Abständen zwischen den Energieniveaus und es ergibt sich eine Nullpunktsenergie $G(0)$ von $\frac{1}{2} \omega_{\mathrm{e}}$.

Die zweite Annahme in der doppelt harmonischen Näherung ist, dass auch das Dipolmoment sich nur linear mit der Auslenkung ändert - die Dipolfunktion also keine Krümmung aufweist. ${ }^{22}$ In Formel 2.2 entspricht das der ausschließlichen Betrachtung des Gliedes 1. Ordnung. Durch diese elektrische Harmonizität lassen sich die Auswahlregeln für den harmonischen Oszillator angewendet auf Moleküle ableiten. Da die Matrixelemente $\left\langle\Psi_{\mathrm{v}^{\prime \prime}}\left|\left(r-r_{\mathrm{e}}\right)\right| \Psi_{\mathrm{v}^{\prime}}\right\rangle$ für $\left|\mathrm{v}^{\prime \prime}-\mathrm{v}^{\prime}\right|>1$ verschwinden, sind nur Übergänge mit $\Delta \mathrm{v}=1$ erlaubt. Weiterhin verlangt der Term $\left.\frac{\mathrm{d} \mu}{\mathrm{d} r}\right|_{r}$, dass das Dipolmoment sich während der Schwingung ändern muss, da sonst das resultierende Übergangsdipolmoment gleich 0 ist.

Die doppelt harmonische Näherung ist oft ausreichend für quantenchemische Betrachtungen von Molekülen und Clustern, wenn es um relative Vergleiche geht. Für die Berechnung von Energieunterschieden verschiedener Moleküle oder Cluster ist die Betrachtung der harmonischen Nullpunktsenergien eine erste Näherung. Und auch die relativen Unterschiede der Rotverschiebungen von Hydrid-Streckschwingungen durch die Ausbildung von Wasserstoffbrücken - eine typische experimentelle Größe bei der Untersuchung entsprechender Cluster - werden ausreichend beschrieben. 


\subsection{Modelle für Molekülschwingungen}

Wird die harmonische Näherung verlassen, so werden Schwingungspotentiale, die von der Parabelform abweichen, und Dipolfunktionen, die nichtlinear sind, erlaubt. Das bekannteste Beispiel für ein anharmonisches Schwingungspotential ist der Morseoszillator. ${ }^{20}$ Dieser ist gültig für zweiatomige Moleküle und bietet eine realistischere Beschreibung bei großen Auslenkungen aus der Ruhelage. Zum einen wird im Morsepotential die Dissoziation bei großen Bindungsabständen zugelassen, zum anderen ergibt sich eine stärkere Repulsion der Kerne bei kurzen Entfernungen. Außerdem sind für den Morseoszillator die zugehörigen Schwingungswellenfunktionen analytisch zugänglich. Die Schwingungsterme (Formel 2.3) werden um den Beitrag $-\omega_{\mathrm{e}} x_{\mathrm{e}}\left(\mathrm{v}+\frac{1}{2}\right)^{2}$ der Anharmonizitätskonstante $\omega_{\mathrm{e}} x_{\mathrm{e}}$ erweitert. Diese führt zu einem sinkenden Abstand der Energieniveaus mit steigender Quantenzahl, was eine Konsequenz aus der mechanischen Anharmonizität - also der zunehmenden Verbreiterung des Potentials mit höheren Energien gegenüber dem harmonischen Oszillator - ist.

Für polyatomare Moleküle mit $n$ Schwingungen muss neben der intrinsischen Anharmonizität einer einzelnen Schwingung auch die anharmonische Wechselwirkung mit den anderen Schwingungsmoden beachtet werden: ${ }^{19}$

$$
G\left(\mathrm{v}_{1}, \mathrm{v}_{2}, \ldots \mathrm{v}_{n}\right)=\sum_{i}^{n} \omega_{i}\left(\mathrm{v}_{i}+\frac{1}{2}\right)+\sum_{i \leq j}^{n} x_{i, j}\left(\mathrm{v}_{i}+\frac{1}{2}\right)\left(\mathrm{v}_{j}+\frac{1}{2}\right)
$$

$\omega_{i}$ ist die harmonische Wellenzahl einer Schwingung und entspricht $\omega_{\mathrm{e}}$ des harmonischen Oszillators. Die diagonalen Anharmonizitäten $x_{i, i}(i=j)$ entsprechen den Anharmonizitätskonstanten $-\omega_{\mathrm{e}} x_{\mathrm{e}}$ des Morseoszillators und weisen somit in den meisten Fällen negative Werte auf. Die außerdiagonalen Terme (Kreuzterme) $x_{i, j}(i \neq j)$ beschreiben die Wechselwirkungen der verschiedenen Schwingungsmoden untereinander. Dementsprechend ergibt sich für die Wellenzahl eines Fundamentalübergangs:

$$
\tilde{\nu}_{i}(1 \leftarrow 0)=G\left(\mathrm{v}_{i}=1\right)-G\left(\mathrm{v}_{i}=0\right)=\omega_{i}+2 \cdot x_{i, i}+\frac{1}{2} \sum_{i<j}^{n} x_{i, j}
$$

Die diagonalen Anharmonizitätskonstanten lassen sich spektroskopisch nach Formel 2.4 aus Oberton- $\left(\tilde{\nu}_{i}(2 \leftarrow 0)=G\left(\mathrm{v}_{i}=2\right)-G\left(\mathrm{v}_{i}=0\right)\right)$ und Fundamentalwellenzahl einer Schwingung gewinnen,

$$
x_{i, i}=\frac{1}{2}\left(\tilde{\nu}_{i}(2 \leftarrow 0)-2 \cdot \tilde{\nu}_{i}(1 \leftarrow 0)\right)
$$

oder aus der Differenz zwischen der Fundamentale einer zugehörigen heißen Bande. Die auBerdiagonalen Anharmonizitäten können aus Kombinationsbanden, beispielsweise einem Zweiquantenübergang $\tilde{\nu}_{i+j}(1 \leftarrow 0,1 \leftarrow 0)=G\left(\mathrm{v}_{i}=1, \mathrm{v}_{j}=1\right)-G\left(\mathrm{v}_{i}=0, \mathrm{v}_{j}=0\right)$ nach

$$
x_{i, j}=\tilde{\nu}_{i+j}(1 \leftarrow 0,1 \leftarrow 0)-\tilde{\nu}_{i}(1 \leftarrow 0)-\tilde{\nu}_{j}(1 \leftarrow 0)
$$

und den zugehörigen Fundamentalübergängen erhalten werden. Alternativ sind auch sie aus heißen Übergängen zugänglich.

Die elektrische Anharmonizität entspricht einer nichtlinearen Dipolfunktion. Es ergibt sich 


\section{Grundlagen}

aus dieser Betrachtung eine Erweiterung der Auswahlregeln auf Grundlage von Formel 2.2. Das Dipolmoment muss sich immer noch entlang der Normalkoordinate ändern, aber Obertöne sind erlaubt. Die Intensitäten sind nun von der Steigung und der Krümmung der Dipolfunktion abhängig, ferner auch von den Matrixelementen der Wellenfunktionen, die je nach Tiefe und Breite des Potentials variieren. Für Fundamentalübergänge dominiert in der Regel die Steigung der Dipolfunktion und damit das Glied 1. Ordnung, während für Obertöne von Hydridschwingungen die Beiträge der Glieder 1. und 2. Ordnung etwa gleich groß sind und sich gegenseitig verstärken oder neutralisieren können. Allgemein werden geringere Intensitäten für Obertöne beobachtet, was vor allem durch kleinere Matrixelemente $\left\langle\Psi_{\mathrm{v}^{\prime \prime}}\left|\left(r-r_{\mathrm{e}}\right)\right| \Psi_{\mathrm{v}^{\prime}}\right\rangle$ für Obertonübergänge erklärt werden kann.

In harmonischen Schwingungsvorhersagen wird nicht die gesamte Potentialhyperfläche untersucht, sondern aus Kostengründen das System nur am Minimum betrachtet. Die analytisch oder numerisch zugängliche zweite Ableitung nach den Auslenkungen der Atome am Potentialminimum erlaubt dann die Konstruktion eines harmonischen Potentials. Aus den Ableitungen ergeben sich für die Auslenkungen der Atome Kraftkonstanten. Aus diesen lassen sich die Normalmoden mit den zugehörigen Schwingungsamplituden der Atome und Schwingungsfrequenzen erhalten. ${ }^{20}$ Es resultieren also aus den zweiten Ableitungen des Potentials die Normalkoordinaten und harmonischen Wellenzahlen der Molekülschwingungen. Die Übergangsdipolmomente ergeben sich nach Formel 2.2 aus dem Matrixelement für Fundamentalübergänge und der Steigung der Dipolfunktion, welche sich analytisch aus der Ableitung der Energie nach der Normalkoordinate und dem elektrischen Feld des Moleküls ergibt ${ }^{23}$ oder sich numerisch berechnen lässt. Die Matrixelemente ergeben sich aus den Normalkoordinaten der Schwingung.

Für eine anharmonische Schwingungsanalyse ${ }^{24}$ kann beispielsweise die Potentialhyperfläche des Systems berechnet und die Schrödingergleichung der Kernbewegungen gelöst werden. ${ }^{25-27}$ Effizienter ist jedoch ein störungstheoretischer Ansatz. ${ }^{21,28}$ Dieser wird als Schwingungsstörungstheorie zweiter Ordnung (vibrational second-order perturbation theory, VPT2) bezeichnet. Bei dieser Methode wird die doppelt harmonische Näherung als Term 0. Ordnung um zwei Korrekturterme erweitert, die die harmonischen Werte um die anharmonischen Beiträge korrigieren.

Für den Beitrag der mechanischen Anharmonizität auf die Wellenzahlen werden numerisch die dritten und ein Teil der vierten Ableitungen des Potentials nach den Normalkoordinaten aus der harmonischen Analyse ermittelt. ${ }^{29}$ Mit ihnen können die diagonalen und außerdiagonalen Anharmonizitätskonstanten der Schwingungen berechnet werden. Außerdem sind Fermiresonanzen und die Wechelwirkung der Schwingungen mit der Rotation des Moleküls in Form von Zentrifugaldehnungskonstanten und Corioliskopplungen möglich. ${ }^{29}$

Für die Berechnung der elektrischen Anharmonizität muss zusätzlich die zweite (1. Ordnung) und dritte Ableitung (2. Ordnung) des Dipolmoments nach den Normalkoordinaten betrachtet werden. Da die Wellenfunktionen des anharmonischen Potentials ebenfalls störungstheoretisch aus den Wellenfunktionen des harmonischen Oszillators konstruiert werden, können die Matrixelemente aus den harmonischen Wellenzahlen der Normalmoden und den Ableitungen des Potentials nach den Normalkoordinaten berechnet werden. ${ }^{30}$ Die entsprechenden Zusammenhänge sind für Fundamentalen, erste Obertöne und Kombinationsbanden (aus zwei Fundamentalen) gegeben, ${ }^{21,30,31}$ sodass die Wellenfunktionen nicht explizit ermittelt werden müssen. Für die Berechnung des anharmonischen Übergangsdipolmoments werden also die Einflüsse der elektri- 
schen und mechanischen Anharmonizität beachtet. Auf analoge Weise sind auch anharmonische VCD- und Ramanintensitäten störungstheoretisch zugänglich. ${ }^{21}$

\subsection{FTIR-Spektroskopie Wasserstoffbrücken-gebundener Cluster in Überschallexpansionen}

Wasserstoffbrücken ${ }^{5,32,33}$ werden zwischen einer Hydridgruppe $(\mathrm{X}-\mathrm{H})$ mit einem elektronegativen Atom $\mathrm{X}$ als Wasserstoffbrückendonor und einer elektronegativen Akzeptorgruppe $\mathrm{Y}$ ausgebildet. Es handelt sich bei ihnen um nicht-kovalente attraktive Wechselwirkungen ${ }^{34,35}$ zwischen diesen Gruppen. Die Bindungsenergie von Wasserstoffbrücken ist geringer als die von kovalenten Bindungen und hängt von der Wasserstoffbrückengeometrie, die vorzugsweise eine lineare $\mathrm{X}-\mathrm{H} \cdots \mathrm{Y}$ Anordnung aufweisen sollte, und der Donor- sowie Akzeptorstärke der involvierten Gruppen ab.

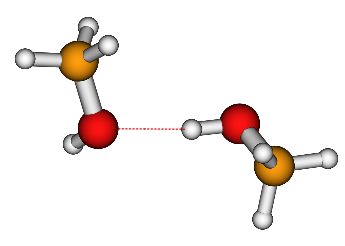

21.4

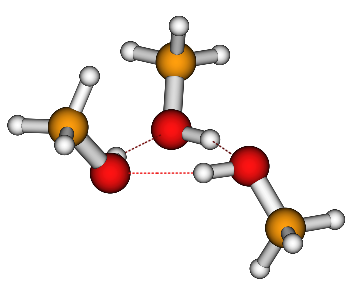

65.7

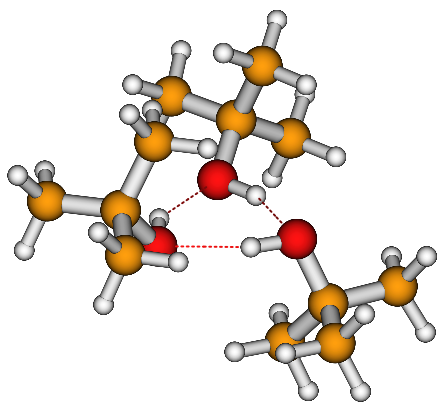

80.3

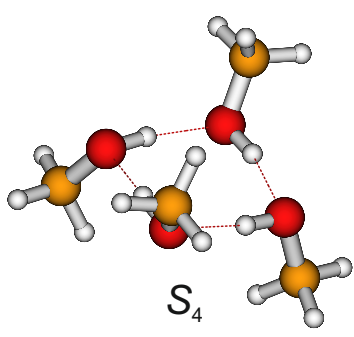

120.3

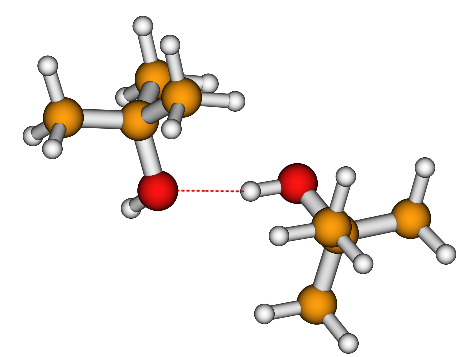

29.2

Abbildung 2.1.: Strukturen der stabilsten Dimere, Trimere und Tetramere von Methanol ${ }^{36}$ und tert-Butylalkohol ${ }^{37}$ auf B3LYP-D3/6-311+G(2d,p) Niveau und Dissoziationsenergien $D_{0}$ in $\mathrm{kJ} / \mathrm{mol}$ unter Berücksichtigung der harmonischen Nullpunktsenergien. 


\section{Grundlagen}

In dieser Arbeit werden Systeme mit Hydroxylfunktionen $(\mathrm{X}=\mathrm{O}, \mathrm{Y}=\mathrm{O})$ untersucht. Neben Wasser $^{38-40}$ sind vor allem Alkohole ${ }^{6,7}$ die einfachsten Moleküle, die O-H ‥ O Wasserstoffbrücken ausbilden. Als Beispiele für kleine Alkoholcluster sind in Abbildung 2.1 die stabilsten Dimere, Trimere und Tetramere von Methanol und tert-Butylalkohol dargestellt. In den Dimeren liegt nur eine intermolekulare Wasserstoffbrücke vor, es kann also zwischen einem Donor- und Akzeptormolekül unterschieden werden. Die Trimere und Tetramere bilden ringförmige Wasserstoffbrückenstrukturen aus, in denen jedes Molekül jeweils der Donor und Akzeptor einer Wasserstoffbrücke ist. Die energetische Bevorzugung dieser Strukturen liegt in der kooperativen Wechselwirkung in den Ringstrukturen begründet, die durch die sich gegenseitig verstärkende Wirkung der Induktion in diesen Geometrien hervorgerufen wird. Die Kooperativität zeigt sich in der steigenden Dissoziationsenergien je Wasserstoffbrücke von den Dimeren zu den Tetrameren. Die Stärke der Wasserstoffbrücken nimmt von den Methanol- zu den tert-Butylalkoholclustern zu, was mit der größeren Akzeptorstärke von tert-Butylalkohol erklärt werden kann. ${ }^{41}$

Die Ausbildung von Wasserstoffbrücken geht mit der Schwächung der $\mathrm{O}-\mathrm{H}$ Bindung des Wasserstoffbrückendonors einher. Spektroskopisch manifestiert sich diese Schwächung in einer niedrigeren Wellenzahl der O-H Streckschwingungsübergang des Donors. ${ }^{3,6,7}$ Gleichzeitig wird die $\mathrm{O}-\mathrm{H}$ Streckschwingung anharmonischer. ${ }^{7,10,11,42,43}$ Die spektrale Verschiebung der Donorbande gegenüber der freien $\mathrm{O}-\mathrm{H}$ Bindung ist die wichtigste spektroskopische Größe bei der Untersuchung von $\mathrm{O}-\mathrm{H} \cdots \mathrm{O}$ Wasserstoffbrücken-gebundenen Clustern. ${ }^{6,7,9,36,37,44-47}$ Die gute Detektierbarkeit von Alkoholclustern und verwandten Systemen in Absorptionsspektren liegt an einer erhöhten Intensität der $\mathrm{O}-\mathrm{H}$ Streckschwingungsfundamentalen in Wasserstoffbrücken. Dies liegt an der Polarisierung der $\mathrm{O}-\mathrm{H}$ Bindung des Donors und der daraus resultierenden steileren Dipolfunktion entlang der $\mathrm{O}-\mathrm{H}$ Normalkoordinate. Für Obertöne wird eine solche Intensitätsverstärkung nicht beobachtet. ${ }^{7,11,43}$ Die Intensitätseffekte sind dort auf Grund der subtilen Wechselwirkung zwischen Steigung und Krümmung der Dipolfunktion geringer. ${ }^{48}$ In symmetrischen Clustern oder Systemen, deren Wasserstoffbrücken nahezu Symmetrie aufzeigen (beispielsweise die lokale näherungsweise $C_{3} / C_{3 \mathrm{~h}}$ Symmetrie der Wasserstoffbrücken in den Alkoholtrimeren), ergeben sich oft komplementäre Spektren mittels Ramanspektroskopie. Die Kombination von Raman- und IR-Spektroskopie bietet häufig einen fruchtbaren Ansatz zur spektroskopischen Charakterisierung von Wasserstoffbrücken-gebundenen Clustern. ${ }^{7,36,49}$

Beliebte experimentelle Methoden zur Messung der Absorptionsspektren verwenden oft IRLaser zur Anregung der O-H Streckschwingungen in Clustern. Die Detektion kann über CavityRingdown Spektroskopie ${ }^{50}$ geschehen oder selektiv für einzelne Clustergrößen bei Doppelresonanzmethoden mit gekoppelter Massenspektrometrie ${ }^{45,51}$ und Streuexperimenten ${ }^{44}$ vorgenommen werden. In dieser Arbeit wird Fourier-Transformations-Infrarot- (FTIR) -spektroskopie ${ }^{52-54}$ zur Messung der Infrarotspektren verwendet. Dazu werden thermische Strahler als Breitbandquellen zur Anregung der Molekül- und Clusterschwingungen genutzt. Das Infrarotlicht wird durch ein Interferometer geleitet und das Spektrum zunächst in Form eines Interferogramms aufgenommen. Durch diese Methode werden zu jedem Zeitpunkt der Messung Informationen über den gesamten Spektralbereich gesammelt, was einen deutlichen Zeitvorteil (Multiplexvorteil) ${ }^{54}$ gegenüber Methoden bietet, bei den die einzelnen Wellenlängen sequentiell gemessen wird. Da kein Monochromator, der enge Spaltblenden aufweisen würde, vorhanden ist, können relativ große Lochblenden verwendet werden. Dadurch wird der Lichtdurchsatz gegenüber 


\subsection{FTIR-Spektroskopie Wasserstoffbrücken-gebundener Cluster in Überschallexpansionen}

Rastermethoden mit Breitbandlichtquellen erhöht (Jacquinot-Vorteil). FTIR Spektrometer sind intern durch einen HeNe-Laser kalibriert, der zur Positionsbestimmung der Interferometerspiegel genutzt wird. Dadurch ergibt sich eine sehr genaue Wellenzahlstabilität und -genauigkeit der FTIR Spektren (Connes-Vorteil). Die Auflösung der Spektren ist umgekehrt proportional zum zurückgelegten Weg des beweglichen Interferometerspiegels von der Nullposition, ${ }^{22}$ sodass also die Messzeit zunimmt, je besser die Auflösung gewählt wird. Ferner wird die Auflösung durch die Größe der Lochblende (Apertur) begrenzt. ${ }^{7}$ Das aufgenommene Interferogramm wird durch eine Fourier-Transformation in das Spektrum umgewandelt.

In der warmen Gasphase liegen Cluster nur in geringen Mengen vor und ihre thermische Anregung führt zu einer deutlichen Verbreiterung und Verschiebung ihrer Banden gegenüber den Bandenzentren. ${ }^{7,40}$ Deshalb werden sie bevorzugt in Molekularstrahlen und Überschallexpansionen ${ }^{54-56}$ untersucht. Bei letzteren werden sie mit einem Trägergas (z.B. Helium, Argon oder Neon) gemischt und von einem hohen Stagnationsdruck durch eine kleine Düse in ein Vakuum unter isentropen Bedingungen expandiert. Ist die Düsenöffnung größer als die mittlere freie Weglänge der Gase, kommt es zu Beginn der Expansion zu zahlreichen Stößen zwischen den Molekülen und Gasatomen. ${ }^{54}$ Diese sind wichtig zur Kühlung (Zweierstöße) und Clusterbildung (Dreierstöße). Es entsteht ein gerichteter Gasfluss der sich mit Überschallgeschwindigkeit ausbreitet, bis er in Stoßfronten gegen den Hintergrunddruck wieder kollabiert. Der Abstand der Stoßfronten zur Düse ist von der Geometrie der Letzteren und dem Verhältnis zwischen Stagnations- und Hintergrunddruck abhängig. Nach dem Bereich vieler Stöße nahe der Düse folgt ein Bereich weniger Stöße (,zone of silence“) auf Grund der zunehmenden Verdünnung des Gases und seiner schmalen Geschwindigkeitsverteilung. Expansionen aus Schlitzdüsen weisen einen geringeren Dichteabfall auf als solche aus Lochdüsen und sind deshalb für die Clusterspektroskopie besser geeignet. Außerdem erlaubt der Betrieb in Form kurzer Pulse und langer Evakuierungszeiten die Verwendung großer Düsen, was die Empfindlichkeit der Messungen deutlich verbessert. ${ }^{7,57}$

Während der Überschallexpansion wird die Energie der inneren Molekülfreiheitsgrade in kinetische Energie umgewandelt, wodurch sich die Moleküle stark abkühlen. Dadurch wird zum einen die Clusterbildung begünstigt. Zum anderen vereinfachen sich ihre Schwingungsspektren, da ihre angeregten Rotations- und Schwingungszustände entvölkert werden. In Überschallexpansionen liegen keine Gleichgewichtsbedingungen vor, sodass ihnen nur formal Temperaturen zugeordnet werden können. Außerdem liegen für die verschiedenen Freiheitsgrade unterschiedliche formale Temperaturen vor. Am stärksten werden die Translationsfreiheitsgrade gekühlt, gefolgt von den Rotationen. Die Schwingungen weisen noch höhere Temperaturen auf. ${ }^{58}$ Für die Konformationen von Molekülen und Clustergeometrien in Überschallexpansionen sind vor allem die kinetischen Barrieren für deren Umwandlungen entscheidend. Sind sie niedrig genug, relaxieren die Systeme in das globale Minimum. ${ }^{46}$ Bei zu hohen kinetischen Barrieren können die Moleküle und Cluster aber in ungünstigen Strukturen ausgefroren werden. Die Verwendung schwerer Trägergase wie Argon und Neon hilft gegenüber Heliumexpansionen oft bei der Relaxation der Systeme in die stabilsten Geometrien. ${ }^{59}$ In der ,zone of silence“ liegen die Moleküle und Cluster isoliert im Vakuum vor und können also ohne Umgebungseinflüsse untersucht werden. 



\section{Experimentelles}

\subsection{Filet-Jet}

Der Filet-Jet ist besonders gut geeignet um die Schwingungen von Molekülen und kleinen Clustern in Überschallexpansionen in vielen unterschiedlichen Wellenzahlbereichen zu untersuchen. Das liegt an den Breitbandlichtquellen und der inhärenten Messmethode der verwendeten FTIRSpektroskopie, das gesamte Spektrum gleichzeitig zu messen - im Gegensatz zu Methoden, bei denen die zu messenden Wellenlängen nacheinander durchgestimmt werden. So wird außerdem die Korrelation von Signalintensitäten in unterschiedlichen Spektralbereichen ermöglicht, was beispielsweise in Laserexperimenten mit schmaler spektraler Bandbreite nicht so leicht gelingt. Die Verwendung von Bandpassfiltern ermöglicht die Konzentration auf ausgewählte Spektralbereiche unter Verbesserung des Signal-zu Rauschen Verhältnisses, da sie die Vergrößerung der Apertur des Spektrometers erlauben. Gleichzeitig bietet der Filet-Jet durch seine namensgebende lange, aber schmale Schlitzdüse (fine but lengthy) einen großen Absorptionsweg durch die untersuchten Überschallexpansionen und damit eine hohe Empfindlichkeit für die teils sehr schwachen Signale in den verschiedenen Spektralbereichen. Durch den schmalen Spalt wird gegenüber breiteren Schlitzdüsen die Bildung großer Cluster unterdrückt und Dimere, Trimere und Tetramere werden eher bevorzugt.

Die vergleichsweise großen Abmessungen der Schlitzdüse unterbinden allerdings weitgehend die Möglichkeit einer Beheizung der Apparatur, was die Experimente am Filet-Jet auf Substanzen mit ausreichend hohem Dampfdruck bei Raumtemperatur beschränkt. Größenselektive Spektren der gemessenen Cluster, wie in Experimenten die beispielsweise Massenspektrometrie mit Schwingungsspektroskopie koppeln, können zwar nicht erreicht werden, jedoch lassen sich die Banden von Monomeren bis Tetrameren in den meisten Systemen gut anhand der Konzentrationsabhängigkeit ihres Signalwachstums unterscheiden - wenn sie im Spektrum gut separiert sind. Da nur kurze Gaspulse am Filet-Jet möglich sind, kann die Auflösung der verwendeten FTIR Spektren ohne Einsatz von Segmentierungstechniken ${ }^{60}$ nicht viel besser als $0.5 \mathrm{~cm}^{-1}$ sein, wobei aber für die meisten Fragestellungen eine Auflösung von $2 \mathrm{~cm}^{-1}$ völlig ausreicht. ${ }^{7}$

Der Filet-Jet wurde im Zuge der Doktorarbeit von Nicole Borho ${ }^{62}$ aufgebaut. Das aktuell verwendete Bruker IFS 66v/S Spektrometer wurde von Susanne Hesse ${ }^{63}$ zur Apparatur hinzu gefügt. In dieser Arbeit wurden vor allem Verbesserungen für Messungen im Nah-und Ferninfrarotbereich vollzogen. Dies geschah vor allem durch den Einsatz neuer empfindlicher Detektoren für diese Spektralbereiche (Nahinfrarot: InGaAs, Ferninfrarot: Bolometer). Außerdem konnte die untere Grenze des Messbereichs durch Verwendung von CsI-Optiken und eines Mylar Strahlteilers von 400 auf $200 \mathrm{~cm}^{-1}$ gesenkt werden.

Das zu untersuchende Gasgemisch wird an der Filet-Jetapparatur (s. Abbildung 3.1) in ei- 


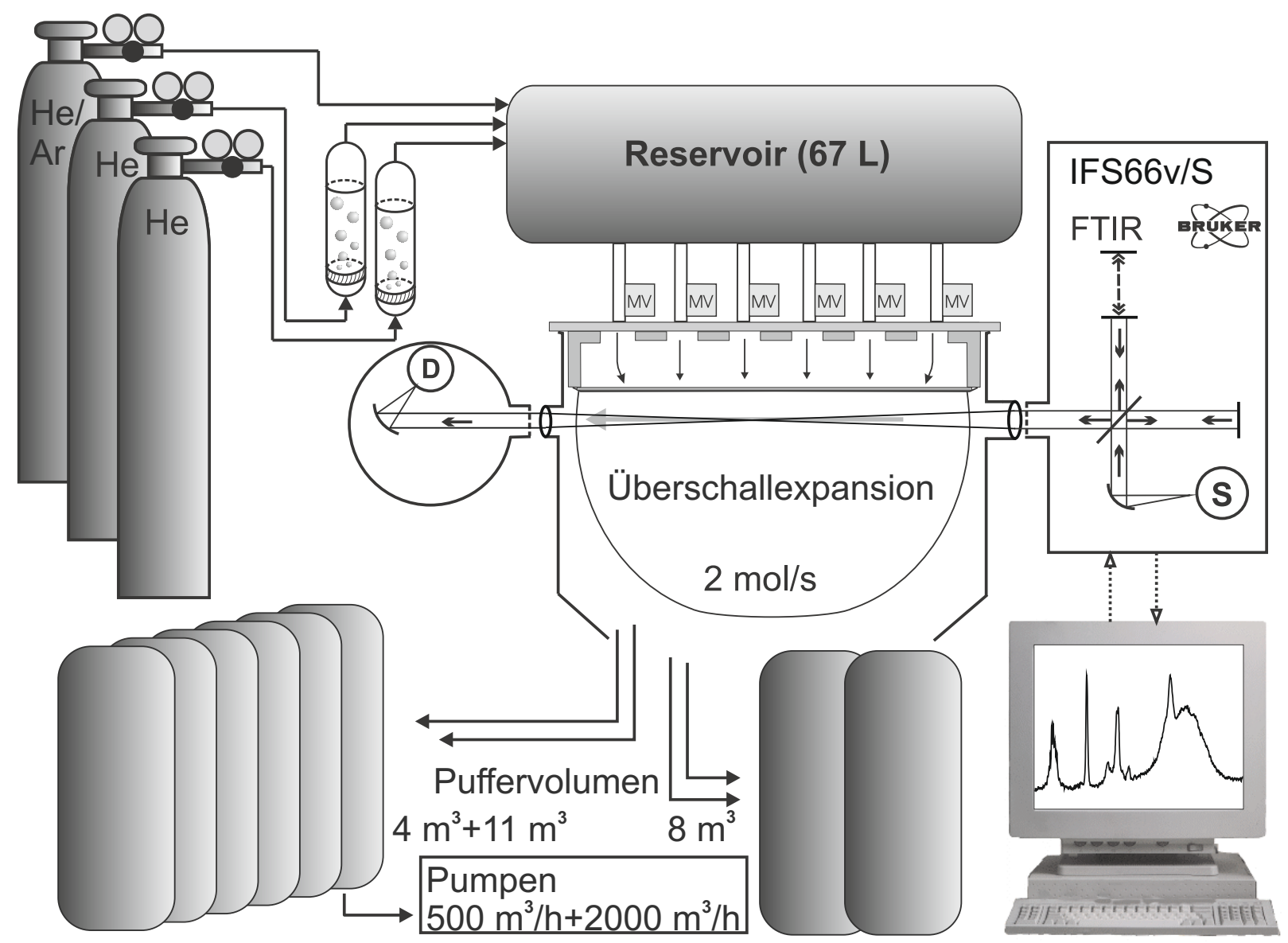

Abbildung 3.1.: Aus Referenz 61 übernommene und modifizierte Darstellung der FiletJetapparatur.

nem 671 umfassenden Reservoir gespeichert. Dieses kann über drei separate Zuleitungen befüllt werden. Zwei der Zuleitungen führen durch temperierbare Sättiger, in die flüssige oder feste Substanzen eingebracht werden können. Aus angeschlossenen Gasflaschen wird dann ein Heliumstrom durch die Sättiger, wo dieser sich mit dem gasförmigen Anteil der Probensubstanzen anreichert, in das Reservoir geführt. Der Partialdruck der Probensubstanz im Helium hängt von ihrem Dampfdruck und damit von der Temperatur des Sättigers ab. Über die dritte Zuleitung ist eine zusätzliche Verdünnung des Gasgemisches im Reservoir mit Helium oder eine Zumischung von anderen Gasen wie Argon möglich. Der Gasfluss durch die Zuleitungen in das Reservoir wird über je ein Magnetventil gesteuert. Durch unterschiedlich lange Öffnungs- und Schließzeiten der einzelnen Magnetventile lässt sich die Zusammensetzung des Gasgemisches im Reservoir zusätzlich regulieren.

Vom Reservoir führen sechs Rohre zur Schlitzdüse. Diese besteht aus einer etwa $330 \mathrm{~cm}^{3}$ große Vorkammer, in die die 600 x $0.2 \mathrm{~mm}^{2}$ große Düse eingelassen ist. Die Expansion wird über je ein Magnetventil an den sechs Rohren gesteuert. Nach Öffnung der Ventile expandiert das Gasgemisch durch die Rohre zunächst in die Vorkammer und von dort durch die Schlitzdü- 
se in die zylindrische Jetkammer. Durch zwei Linsen in den Wänden der Jetkammer wird der Strahlengang des Bruker IFS 66v/S FTIR Spektrometer entlang der Schlitzdüse geführt. ${ }^{63} \mathrm{Er}$ gelangt zuvor durch ein Fenster aus dem evakuierten Spektrometer und dann durch eine Linse mit einer Brennweite von $500 \mathrm{~mm}$ in die Jetkammer und verlässt sie auf der gegenüber liegenden Seite durch eine Linse mit einer Brennweite von $250 \mathrm{~mm}$. So wird der kollimierte Strahl aus dem Spektrometer sanft auf einen Brennpunkt in der Expansion fokussiert. Der Spektrometerstrahl wird so nah wie möglich an der Schlitzdüse entlang geführt und sein Durchmesser variiert in der Expansion zwischen etwa 1 und $2 \mathrm{~cm}$. Beim Austritt aus der Probenkammer wird der Strahl wieder rekollimiert und trifft auf einen Spiegel, der ihn auf den Detektor fokussiert. Dieser befindet sich in einer evakuierbaren Detektorkammer, in die der Strahl durch ein Fenster eindringt. Die Zwischenräume zwischen Fenstern und Linsen werden mit trockener Luft gespült. Der gesamte Lichtweg in der Jetkammer beträgt $776 \mathrm{~mm}$.

Um ein ausreichendes Vakuum während der Gasexpansionen zu gewährleisten, ist die Probenkammer an ein kontinuierlich evakuiertes Puffervolumen angeschlossen. Dieses ist durch große Rohrleitungen senkrecht zu Schlitzdüse und Expansionsrichtung angeschlossen. Auf der einen Seite ist ein Puffervolumen von etwa $8 \mathrm{~m}^{3}$ und auf der gegenüberliegenden Seite ein Puffervolumen von etwa $4 \mathrm{~m}^{3}$, das um etwa $11 \mathrm{~m}^{3}$ erweitert werden kann, angeschlossen. ${ }^{62} \mathrm{Am} 4 \mathrm{~m}^{3}$ Puffervolumen befindet sich ein Pumpstand mit einer Leistung von ca. $500 \mathrm{~m}^{3} / \mathrm{h}$, der bei Erweiterung des Puffervolumens um einen weiteren Pumpstand mit einer Leistung von ca. $2000 \mathrm{~m}^{3} / \mathrm{h}$ ergänzt werden kann. Eine umfassende Darstellung der technischen Daten der gesamten Apparatur ist in Referenz 62 enthalten.

Da während der Messungen der Druck in der Probenkammer nicht über 1 mbar steigen sollte, werden die Gasexpansionen in kurzen Pulsen durchgeführt. Diese Pulse werden mit den Scans des Spektrometers synchronisiert. Da sich insbesondere bei Ferninfrarotmessungen durch die geringe Spiegelgeschwindigkeit und bei Messungen mit hoher Auflösung durch den größeren Weg des Interferometerspiegels lange Scanzeiten ergeben, ist für diese eine Verwendung des gesamten Puffervolumens unerlässlich, da sonst der Druck in der Probenkammer während der entsprechend langen Gaspulse zu groß wird.

Eine Messsequenz besteht aus 20 Hintergrundscans die bei ungefähr 0.1 mbar Druck in der Jetkammer aufgenommen werden, es folgen 2 Vorscans, ein Probescan, bei dem die Expansion stattfindet und zwei Nachscans. Um möglichst kurze Scanzeiten des Spektrometers zu erreichen, wird der single-sided fast return Modus des Spektrometers verwendet. Die Magnetventile zur Düse werden etwa $40 \mathrm{~ms}$ vor dem Probescan geöffnet, und nach dem 100 bis $400 \mathrm{~ms}$ dauernden Probescan wieder geschlossen. Darauf folgt eine Pausenzeit von 20 bis $120 \mathrm{~s}$ in denen das Vakuum im Puffervolumen wieder auf 0.1 mbar gebracht wird. Die Synchronisation der Pulse mit den Scans des Spektrometers wird detailliert in Referenz 62 und ferner in Referenz 7 beschrieben. Für die Messungen wurde die Software OPUS 6.0 verwendet. Die Fouriertransformationen wurden mit der Phasenkorrektur „Mertz“, der Apodisierung „Norton-Beer-Medium“ und einem Zero Filling Factor von 4 durchgeführt.

Die verwendeten Spektrometereinstellungen, Lichtquellen, Strahlteiler, optischen Materialien und Detektoren für die einzelnen Spektralbereiche werden in den jeweiligen Kapiteln dieser Arbeit spezifiziert. Ebenfalls sind dort die Sättigertemperaturen und Mischungsverhältnisse bei Befüllung des Reservoirs über mehrere Zuleitungen gegeben. 


\section{Experimentelles}

\subsection{Popcorn-Jet}

Beim Popcorn-Jet wird eine wesentliche kleinere Düse als am Filet-Jet verwendet, die zwei parallele $0.5 \times 10 \mathrm{~mm}^{2}$ Schlitze in einem Abstand von $10 \mathrm{~mm}$ besitzt. Der Strahl des FTIRSpektrometers verläuft senkrecht zu den Schlitzen und zu der Expansionsrichtung. Die zu untersuchende Substanz wird auf Molekularsieb gebracht und mit diesem in der Düse vor den Schlitzen platziert. Der kleinere Aufbau erlaubt die Beheizung der Düse und somit die Messung von schwerflüchtigen Substanzen. Der Aufbau der Popcorn-Jet Apparatur, wie sie für diese Arbeit verwendet wurde, ist in Referenz 64 beschrieben. Modifikationen des Experimentes werden in den Referenzen 65 und 66 wiedergegeben.

\subsection{Detektorenvergleich}

Um die Empfindlichkeit verschiedener Detektoren zu charakterisieren und zu vergleichen wurden systematische Testmessungen ohne Probe durchgeführt. Dazu wurden die Messparameter variiert bis ein möglichst geringes Rauschen in den Spektren erreicht wurde. Um Messungen bei verschiedenen Spiegelgeschwindigkeiten miteinander vergleichen zu können, wurden die Hintergrund- und leeren Probemessungen für jeweils 1 min durchgeführt. Die Messungen wurden am Vertex 70 des Chili-Jet ${ }^{67}$ unter Verwendung des Globar 12V und des KBr-Strahlteilers vorgenommen. Die Parameter der einzelnen Messungen sind in Tabelle 3.1 gegeben.

In Sandwichdetektoren liegen die MCT-Detektoren unter InSb-Elementen. Dadurch wird die eintreffende Infrarotstrahlung vor dem MCT bereits gefiltert und erreicht diesen nur unterhalb von etwa $2000 \mathrm{~cm}^{-1}$. Um den Vergleich mit anderen MCT-Detektoren mit Einzelelementen zu ermöglichen wurde ein Bandpassfilter benutzt, der den Messbereich auf 1100 bis $2260 \mathrm{~cm}^{-1}$ eingrenzt (Filter 4). In Abbildung 3.2 zeigt sich, dass der Sandwichdetektor (,MCT_Sandwich_neu“) gegenüber den Einzelelementdetektoren im Bereich von etwa 1300 bis $1600 \mathrm{~cm}^{-1}$ bessere Messempfindlichkeit bietet. Dafür sinkt seine Empfindlichkeit oberhalb von $1800 \mathrm{~cm}^{-1}$, bedingt durch das InSb-Element, stark ab.

Tabelle 3.1.: Messparameter für den Vergleich von MCT Detektoren: Apertur im Spektrometer, ADC Signal des Interferogrammmaximums, wechselbarer Widerstand im Vorverstärker, Spiegelgeschwindigkeit und elektronischer Tiefpassfilter.

\begin{tabular}{lccccc}
\hline Detektor & $\begin{array}{c}\text { Apertur } \\
/ \mathrm{mm}\end{array}$ & ADC Signal & $\begin{array}{c}\text { Widerstand } \\
/ \mathrm{k} \Omega\end{array}$ & $\begin{array}{c}\text { Spiegelgeschw. } \\
/ \mathrm{kHz}\end{array}$ & $\begin{array}{c}\text { Tiefpassfilter } \\
/ \mathrm{kHz}\end{array}$ \\
\hline MCT_Sandwich_neu & 3.0 & 30245 & 2.0 & 80 & - \\
MCT_2mm_neu & 3.0 & 31750 & 1.0 & 60 & 10 \\
MCT_2mm_alt & 8.0 & 24000 & 1.2 & 80 & 10 \\
\hline
\end{tabular}




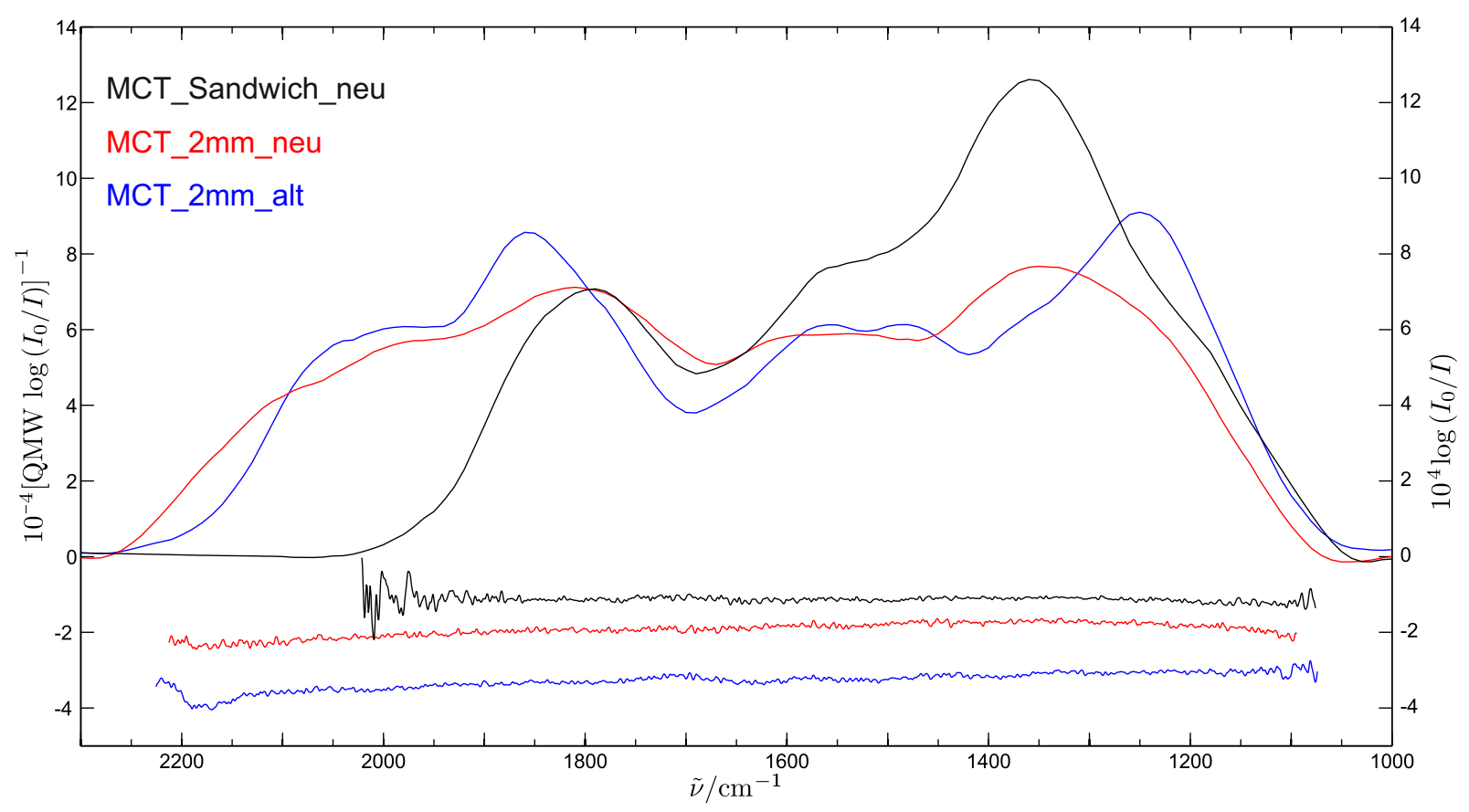

Abbildung 3.2.: Darstellung der Empfindlichkeit (obere Kurven) von MCT-Detektoren mit Filter $4\left(1100-2260 \mathrm{~cm}^{-1}\right)$. Auf der linken Ordinate ist der reziproke quadratische Mittelwert (QMW) der dekadischen Absorbanz log $I_{0} / I$ über je $100 \mathrm{~cm}^{-1}$ von Leermessungen der Detektoren aufgetragen. Stellenweise negative Werte rühren aus einer anschließenden Glättung her. Unten sind die zugehörigen Spektren der Leermessungen in Form der dekadischen Absorbanz dargestellt (rechte Ordinate).

\subsection{Chemikalien}

Die untersuchten Substanzen wurden kommerziell erhalten und ohne weitere Aufarbeitung untersucht. Ihre Hersteller und Reinheiten sind in Tabelle 3.2 angegeben.

\subsection{Quantenchemische Rechnungen}

Alle Rechnungen wurden mit dem Gaussian09, Revision D.01 ${ }^{68}$ Programmpaket durchgeführt. Standardmäßig wurde das B3LYP-D3 Funktional mit dem Basissatz 6-311+G(2d,p) verwendet. Bei B3LYP-D3 wird durch empirische Koeffizienten eine Dispersionskorrektur durchgeführt, ${ }^{69}$ die über Becke-Johnson Dämpfung ${ }^{70}$ bei kurzen Atomabständen korrigiert wird. Für harmonische Frequenzrechnungen reicht eine vorherige Optimierung mit den Standardparametern aus. Nur für Berechnungen von tert-Butylalkohol wurden strengere Optimierungskriterien „fopt=verytight“ und ein feineres Gitter „Int=Superfine“ verwendet, um imaginäre Wellenzahlen zu vermeiden. Dieselben Parameter wurden aus gleichem Grund auch für alle anharmonischen 


\section{Experimentelles}

Tabelle 3.2.: Verwendete Chemikalien, Hersteller und Reinheit.

\begin{tabular}{|c|c|c|c|}
\hline Substanz & CAS-Nr. & Hersteller & Reinheit \\
\hline $\mathrm{HCOOH}$ & $64-18-6$ & Sigma-Aldrich & $>98 \%$ \\
\hline HCOOD & $925-94-0$ & Sigma-Aldrich & $95 \%, 98 \% \mathrm{D}$ \\
\hline $\mathrm{DCOOH}$ & $917-71-5$ & Sigma-Aldrich & $95 \%, 98 \% \mathrm{D}$ \\
\hline DCOOD & $920-42-3$ & Sigma-Aldrich & $95 \%, 98 \% \mathrm{D}$ \\
\hline Argon & $7440-37-1$ & Air Liquide & $99.998 \%$ \\
\hline tert-Butylalkohol & $75-65-0$ & Roth & $\geq 99 \%$ \\
\hline tert-Butylalkohol-OD & $3972-25-6$ & Cambridge Isotope Laboratories & $99 \%$ \\
\hline Ethanol & $64-17-5$ & Roth & $\geq 99.8 \%$ \\
\hline 1,2-Ethandiol & $107-21-1$ & ABCR & $99 \%$ \\
\hline Helium & $7440-59-7$ & Linde & $99.996 \%$ \\
\hline 1,1,1,3,3,3-Hexafluorisopropanol & $920-66-1$ & $\mathrm{ABCR}$ & $99 \%$ \\
\hline $\mathrm{CH}_{3} \mathrm{OH}$ & $67-56-1$ & VWR & $99.9 \%$ \\
\hline $\mathrm{CH}_{3} \mathrm{OD}$ & $1455-13-6$ & euriso-top & $>99.9 \%, 99 \% \mathrm{D}$ \\
\hline $\mathrm{CD}_{3} \mathrm{OH}$ & $1849-29-2$ & euriso-top & $>99.9 \%, 99.8 \% \mathrm{D}$ \\
\hline $\mathrm{CD}_{3} \mathrm{OD}$ & $811-98-3$ & euriso-top & $>99.97 \%, 99.8 \% \mathrm{D}$ \\
\hline Methyllaktat & $547-64-8$ & Fluka & $>97 \%$ \\
\hline$(S)$-Methyllaktat & $27871-49-4$ & Fluka & $98 \%, 97 \%$ ee \\
\hline 1,2-Propandiol & $57-55-6$ & Acros Organics & $>99 \%$ \\
\hline$(S)$-1,2-Propandiol & $4254-15-3$ & ABCR & $99 \%$ \\
\hline (R)-1,2-Propandiol & $4254-14-2$ & ABCR & $98 \%$ \\
\hline 2,2,2-Trifluorethanol & $75-89-8$ & ABCR & $99 \%$ \\
\hline 2,2,2-Trifluorethanol-OD & $77568-66-2$ & Aldrich & $99 \%$ OD \\
\hline
\end{tabular}

Rechnungen ${ }^{21,29,31}$ auf B3LYP-D3/6-311+G(2d,p)-Niveau verwendet. Abweichende Rechenmethoden werden explizit im Text der folgenden Kapitel beschrieben. 


\section{4. $\mathrm{O}-\mathrm{H}$ Streckschwingungen}

\subsection{Fundamentalübergänge}

In diesem Abschnitt sollen die Dimere von einfachen, vicinalen Diolen untersucht werden. Diese Verbindungen weisen zwei Hydroxylgruppen auf, sodass in ihren Dimeren bis zu vier Wasserstoffbrücken ausgebildet werden können. An den Beispielen von Ethylenglykol und Propylenglykol werden die Dimere mittels ihrer IR-Signale in Überschallexpansionen im Vergleich mit quantenchemischen Schwingungsvorhersagen untersucht.

\subsubsection{Messbedingungen}

Tabelle 4.1.: Experimentelle Bedingungen der am Filet-Jet aufgenommenen Spektren von Diolen: Stagnationsdruck $p_{\text {st }}$ und Sättigertemperatur $T_{\mathrm{S}}$. Die Konzentrationen der Substanzen im Reservoir wurde aus ihren Gleichgewichtsdampfdrücken bei der Sättigertemperatur und dem Heliumdruck $p_{\mathrm{He}}$ im Sättiger abgeschätzt.

\begin{tabular}{lcrcc}
\hline & $p_{\text {st }} /$ bar & $T_{\mathrm{S}} /{ }^{\circ} \mathrm{C}$ & $p_{\mathrm{He}}$ & Konzentration \\
\hline Ethylenglykol & 0.7 & 20 & 1.1 & $0.007 \%$ \\
Ethylenglykol & 0.7 & 25 & 1.1 & $0.01 \%$ \\
rac.-Propylenglykol $^{(S) \text {-Propylenglykol }}{ }^{a}$ & 1.3 & 20 & 1.6 & $0.007 \%$ \\
\hline
\end{tabular}

${ }^{a}$ zusammen mit Daniel Falkowski aufgenommen $^{71}$

Für die Messungen der $\mathrm{O}-\mathrm{H}$ Streckschwingungen am Filet-Jet wurde im Fall von Propylenglykol ein InSb-Detektor (77 K, „InSb_2mm_neu“) verwendet und für Ethylenglykol das InSbElement eines InSb/MCT Sandwichdetektors ( 77 K, ,Sandwich_neu“). Im Falle von Ethylenglykol wurde für Strahlteiler, Linsen und Fenster $\mathrm{CaF}_{2}$ genutzt, eine Apertur von $4 \mathrm{~mm}$, die Wolframlampe des Spektrometers als Lichtquelle und Filter $11\left(3720-3080 \mathrm{~cm}^{-1}\right)$ verwendet. Für Propylenglykolmessungen wurden ein $\mathrm{CaF}_{2}$ Strahlteiler genutzt sowie $\mathrm{KBr}$ Linsen und Fenster. Es wurde eine Apertur von $5 \mathrm{~mm}$ verwendet und Filter $14+7\left(3900-2950 \mathrm{~cm}^{-1}\right)$. Als Lichtquelle fungierte die Wolframlampe des Spektrometers. Die Bedingungen in den Sättigern, über die das Reservoir befüllt wurde, sind in Tabelle 4.1 zusammengefasst.

Für die Messungen am Popcorn-Jet wurden Strahlteiler, Linsen und Fenster aus $\mathrm{KBr}$ verwendet. Als Lichtquelle wurde der Globar des Spektrometers genutzt. Die Apertur wurde auf $4 \mathrm{~mm}$ gestellt und es wurde Filter $14+7\left(3900-2950 \mathrm{~cm}^{-1}\right)$ verwendet. Zur Aufnahme der Interferogramme kam ein InSb-Detektor (77 K, „InSb_2mm_alt“) zum Einsatz. 


\section{4. $O-H$ Streckschwingungen}

Alle hier vorgestellten Messungen wurden mit einer Spiegelgeschwindigkeit von $80 \mathrm{kHz}$ und einer Auflösung von $2 \mathrm{~cm}^{-1}$ durchgeführt.

\subsubsection{Ethylenglykol}

Ethylenglykol (Ethan-1,2-diol) ist das einfachste vicinale Diol und findet eine breite Anwendung in der chemischen Industrie und im alltäglichen Leben, insbesondere als Frostschutzmittel. ${ }^{72}$

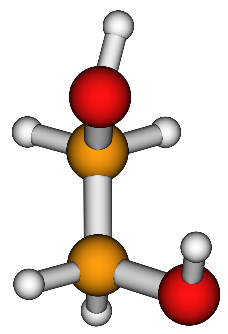

I 0.0

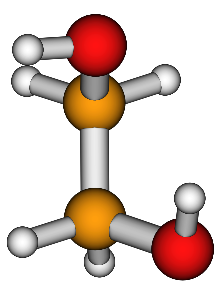

II

1.4

Abbildung 4.1.: Berechnete Strukturen (B3LYP-D3/6-311+G(2d,p)) der zwei günstigsten Konformere von Ethylenglykol. Die relativen Energien sind unter Berücksichtigung der harmonischen Nullpunktsenergien in $\mathrm{kJ} / \mathrm{mol}$ gegeben.

Die Konformere des Monomers wurden in der Gasphase mittels vielfältiger Techniken untersucht, wobei vor allem durch Mikrowellenspektroskopie die Geometrien der beiden günstigsten Konformationen des isolierten Moleküls charakterisiert werden konnten. ${ }^{73-80}$ Die beiden $\mathrm{C}-\mathrm{O}$ Bindungen des Ethylenglykols nehmen in ihnen einen gauche Winkel zueinander ein (Vgl. Abbildung 4.1), ${ }^{73,74}$ wodurch die Ausbildung einer schwachen intramolekularen Wasserstoffbrücke ermöglicht wird. ${ }^{73,74}$ Die beiden stabilsten Konformere unterscheiden sich nur durch die Ausrichtung der freien Hydroxygruppe, die entweder trans (Konformer I) oder gauche (Konformer II) zur C-C Bindung steht. ${ }^{76}$ Der Energievorteil der trans-Stellung der freien $\mathrm{O}-\mathrm{H}$ Gruppe wurde experimentell auf $1.4(4) \mathrm{kJ} / \mathrm{mol}^{76}$ beziehungsweise auf $2.5(5) \mathrm{kJ} / \mathrm{mol}^{80}$ bestimmt. Diese Werte sind vergleichbar mit dem hier berechneten Energieunterschied von $1.4 \mathrm{~kJ} / \mathrm{mol}$ auf B3LYP-D3/6-311+G(2d,p) Niveau unter Berücksichtigung der harmonischen Nullpunktsenergien. Die zunehmend umfassende Zuordnung des Mikrowellenspektrums von Ethylenglykol ${ }^{79,80}$ erlaubte die Detektion des Konformers I im Weltraum. ${ }^{81,82}$

In beiden Konformeren können die Hydroxygruppen eine konzertierte Torsion ausführen, die wieder zum gleichen Konformer führt, aber die Rolle von Protonendonor und -akzeptor vertauscht. Diese Umwandlung wird im Grundzustand durch Tunneln durch die Energiebarrieren realisiert. Dies führt zur Tunnelaufspaltung der Schwingungs-Rotationszustände der einzelnen Konformere. Diese beträgt im Grundzustand für Konformer I $\mathrm{I}^{77} 0.2 \mathrm{~cm}^{-1}$ und auf Grund eines komplexeren Tunnelpfades für Konformer II ${ }^{78}$ nur $0.05 \mathrm{~cm}^{-1}$.

Gasförmiges Ethylenglykol wurde auch in Elektronenbeugungsexperimenten untersucht. So konnte die gauche Stellung der $\mathrm{C}-\mathrm{O}$ Gruppen zueinander ebenfalls nachgewiesen werden. ${ }^{83}$ 


\subsection{Fundamentalübergänge}

Bei erhöhten Temperaturen konnten auch Moleküle, deren $\mathrm{C}-\mathrm{O}$ Gruppen trans zueinander stehen, beobachtet werden. ${ }^{84}$ Diese Konformere sind energetisch $6(2) \mathrm{kJ} / \mathrm{mol}$ ungünstiger ${ }^{84}$ und für Mikrowellenspektroskopie nicht zugänglich, wenn sie Inversionssymmetrie aufweisen.

Durch IR-Absorption wurde Ethylenglykol in der Gasphase zuerst anhand der zweiten Obertöne der $\mathrm{O}-\mathrm{H}$ Streckschwingungen beobachtet. ${ }^{85}$ Es folgte die Untersuchung der Fundamentalübergänge in der gasförmigen und kondensierten Phase. ${ }^{86}$ Jedoch erlaubt die Spektroskopie der Obertöne der $\mathrm{O}-\mathrm{H}$ Streckschwingungen eine bessere Trennung der Banden der zwei günstigsten Konformere und eine Charakterisierung der schwachen intramolekularen Wasserstoffbrücke in der Gasphase, ${ }^{87}$ auch im Vergleich mit anderen Diolen. ${ }^{88}$

Isoliert in Edelgasmatrizen kann das Umwandlungsverhalten von Ethylenglykol untersucht werden. Während sich zunächst nur die beiden stabilsten Konformere in den Matrizen isolieren lassen, ${ }^{89}$ können diese durch Bestrahlung mit Infrarotlicht in andere Konformere umgewandelt werden. ${ }^{89-92}$ Die Anregung der O-H Streckschwingungen führt zur Änderung der Konformation der $\mathrm{O}-\mathrm{C}-\mathrm{C}-\mathrm{O}$ Diederwinkel von gauche zu trans. ${ }^{89,92}$

Die flüssige Phase von Ethylenglykol wurde unter anderem mittels IR Absorption, ${ }^{93-95}$ Beugungsexperimenten ${ }^{96}$ und Molekulardynamiksimulationen ${ }^{97,98}$ untersucht. In ihr liegt ein Gleichgewicht zwischen intra- und intermolekularen Wasserstoffbrücken vor. ${ }^{96}$ Mittels Ramanspektroskopie lässt sich in der Flüssigkeit auch die trans Konformation der C-O Gruppen zueinander beobachten. ${ }^{93,99,100}$ In der kristallinen Form von Ethylenglykol sind alle Hydroxylgruppen in intermolekularen Wasserstoffbrücken gebunden. ${ }^{101}$

In Jetexpansionen wurde mittels Mikrowellenspektroskopie bisher nur das stabilere Konformer I beobachtet, ${ }^{78}$ da die Moleküle zu einem signifikanten Anteil in die stabilste Konformation relaxieren. Auch in den Spektren des Filet-Jet (Abbildung 4.2) zeigen sich nur zwei $\mathrm{O}-\mathrm{H}$ Streckübergänge bei 3689 und $3636 \mathrm{~cm}^{-1}$, die somit Konformer I zugeordnet werden sollten. Das Fundamentalspektrum der Gasphase bei Raumtemperatur ist auf Grund der starken Überlappung der Banden nicht geeignet um diese Zuordnung zu überprüfen. Jedoch lassen sich aus den Obertonspektren ${ }^{87}$ die ungefähren Bandenpositionen der Monomerfundamentalen extrapolieren. Daraus ergeben sich für Konformer I O-H Streckschwingungswellenzahlen bei 3688(1) und $3633(3) \mathrm{cm}^{-1}$ und für Konformer II bei 3654(4) und 3622(1) $\mathrm{cm}^{-1}$. Die Zuordnung von Konformer I zu den beobachteten $\mathrm{O}-\mathrm{H}$ Streckschwingungsfundamentalen in den Spektren der Jetexpansionen stimmt damit überein. Der Übergang bei der niedrigeren Wellenzahl ist der $\mathrm{O}-\mathrm{H}$ Gruppe in der schwachen Wasserstoffbrücke zuzuordnen.

Auf Grund des äußerst geringen Dampfdrucks von Ethylenglykol bei Raumtemperatur können nur vergleichsweise geringe Konzentrationen der Substanz in den Expansionen des Filet-Jet realisiert werden. Trotzdem zeigt sich bei dem höher konzentrierten Spektrum in Abbildung 4.2 eine Clusterbande bei $3513 \mathrm{~cm}^{-1}$, die höchstwahrscheinlich auf das Dimer zurück geht. Dafür spricht die grundsätzliche Annahme, dass zunächst Dimerbanden vor den Banden größerer Aggregate bei Steigerung der Konzentration sichtbar sein sollten. Außerdem können im verwandten Fall des Propylenglykols Dimerbanden an ähnlicher Position beobachtet werden, während die Banden größerer Cluster stärker rotverschoben und deutlich verbreitert erscheinen (siehe folgenden Abschnitt 4.1.3).

In Abbildung 4.3 sind Strukturen einiger Ethylenglykoldimere gezeigt, die auf B3LYP-D3/6$311+G(2 d, p)$ Niveau gefunden wurden. Sie sind nach der Anzahl ihrer intermolekularen Was- 


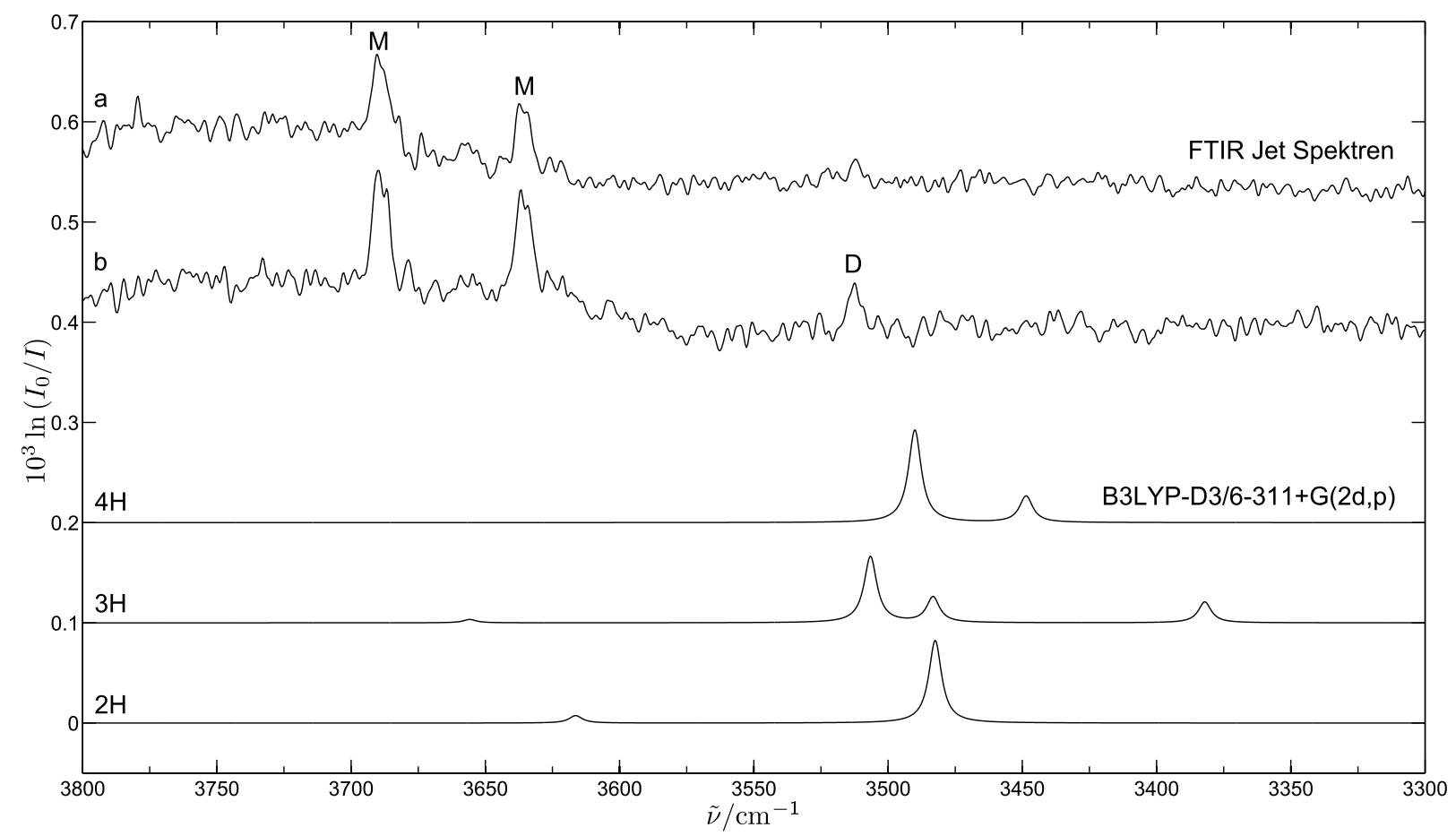

Abbildung 4.2.: FTIR Spektren von Ethylenglykol in den Expansionen des Filet-Jet: a - 0.007\% in 0.7 bar Helium (200 Pulse) und b $-0.01 \%$ in 0.7 bar Helium (100 Pulse). Zum Vergleich sind die harmonischen Vorhersagen zu den Dimerstrukturen $4 \mathrm{H}, 3 \mathrm{H}$ und $2 \mathrm{H}$ von Ethylenglykol auf B3LYP-D3/6-311+G(2d,p) Niveau abgebildet. Die Intensitäten der Rechnungen wurden dazu für eine angenommene Dimerkonzentration von 10\% der Monomerkonzentration aufgetragen und die Wellenzahlen mit dem Faktor 0.9569 skaliert.

serstoffbrücken benannt. Die mit Abstand stabilste Struktur 4H weist vier intermolekulare Wasserstoffbrücken auf. Danach folgt die zweitstabilste Struktur 3H mit drei intermolekularen Wasserstoffbrücken und einer freien Hydroxylgruppe, die unter Berücksichtigung der harmonischen Nullpunktsenergie $7.5 \mathrm{~kJ} / \mathrm{mol}$ weniger stabil ist. Außerdem ist die Struktur $2 \mathrm{H}$ mit nur zwei intermolekularen Wasserstoffbrücken gezeigt. In ihr enthalten beide Moleküle je zusätzlich eine intramolekulare Wasserstoffbrücke, so wie im Fall der isolierten Monomere. In der energetischen Reihenfolge auf B3LYP-D3/6-311+G(2d,p) Niveau liegt diese Struktur jedoch nur an sechster Stelle, da sich zwischen ihr und $3 \mathrm{H}$ noch drei weitere Strukturen mit drei intermolekularen Wasserstoffbrücken befinden. Es ist möglich, dass intramolekulare Wasserstoffbrücken in Jetexpansionen bei der Clusterbildung erhalten bleiben, wenn die kinetischen Barrieren zu hoch sind um sie zu brechen oder die möglichen intermolekularen Wasserstoffbrücken zu schwach sind um in Konkurrenz zu treten. Im Falle von Ethylenglykol ist für die Umwandlung der Dimere ineinander nur die Torsion der $\mathrm{O}-\mathrm{H}$ Gruppen und Verlagerungen der Monomereinheiten notwendig, sodass die kinetischen Barrieren tief genug liegen sollten um eine Relaxation in das globale Minimum zuzulassen. Bei diesem handelt es sich mit hoher Wahrscheinlichkeit um das Dimer 4H, 


\subsection{Fundamentalübergänge}

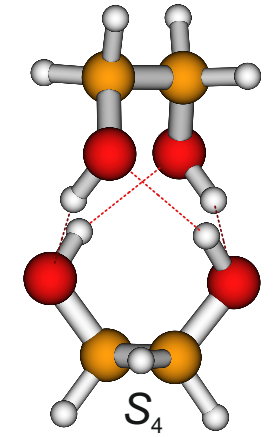

51.3

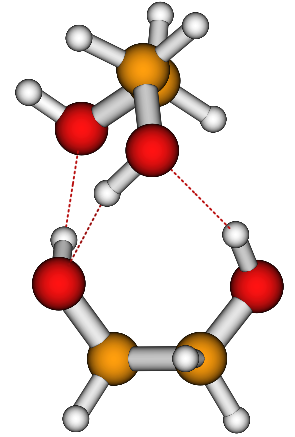

43.8

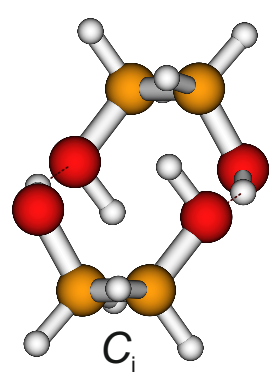

40.5

$4 \mathrm{H}$

$3 \mathrm{H}$

$2 \mathrm{H}$

Abbildung 4.3.: Die zwei stabilsten Dimere von Ethylenglykol $(4 \mathrm{H}$ und $3 \mathrm{H})$ und eine $2 \mathrm{H}$ Struktur auf B3LYP-D3/6-311+G(2d,p) Niveau und Dissoziationsenergien $D_{0}$ in $\mathrm{kJ} / \mathrm{mol}$ unter Berücksichtigung der harmonischen Nullpunktsenergien.

da sein berechneter Energievorteil sehr deutlich gegenüber allen anderen Strukturen ist. Ob das zweitstabilste Dimer aber tatsächlich die in Abbildung 4.3 gezeigte 3H Struktur ist, kann nicht mit Sicherheit gesagt werden, da die Anzahl möglicher Dimerstrukturen mit sinkender Dissoziationsenergie immer stärker zunimmt und rechnerisch drei Strukturen gefunden wurden, die maximal $2.5 \mathrm{~kJ} / \mathrm{mol}$ gegenüber $3 \mathrm{H}$ benachteiligt sind.

Die energetische Reihenfolge der Dimerstrukturen ist stark vom verwendeten Rechenniveau abhängig. So wurden in einer früheren Studie auf MP2/6-311++G**- und M06-2X/6-311++G**Niveau drei Dimerstrukturen gefunden, wobei die stabilste drei und die anderen beiden Strukturen je zwei intermolekulare Wasserstoffbrücken aufweisen. ${ }^{102}$ Von ihnen entspricht jedoch keine den in Abbildung 4.3 gezeigten Strukturen. In einer weiteren Studie wurde auf B3LYP/6$31+G^{* *}$ Niveau ein Dimer mit drei intermolekularen Wasserstoffbrücken als stabilste Struktur präsentiert, gefolgt von Dimeren mit zwei bzw. drei Wasserstoffbrücken zwischen den beiden Molekülen. ${ }^{96} \mathrm{Ob}$ und mit welcher Energie die Strukturen in Abbildung 4.3 gefunden wurden, kann nicht nachvollzogen werden. Unter Verwendung des HF/6-31+G**-Niveaus zur Optimierung wurden drei Dimerstrukturen gefunden, die ein oder zwei zwischenmolekulare Wasserstoffbrücken aufweisen. ${ }^{103}$ Andere Studien haben ausgewählte Dimere mit nur einer intermolekularen Wasserstoffbrücke betrachtet. ${ }^{104,105}$ Fraglich ist, ob die Suche nach Dimerstrukturen in den Studien mit insgesamt nur drei gefundenen Dimeren ${ }^{102,103}$ umfangreich genug war, um die Potentialfläche des Dimers ausreichend abzutasten. In den hier durchgeführten Rechnungen auf B3LYP-D3/6-311+G(2d,p) Niveau konnten allein im Energieintervall von $15 \mathrm{~kJ} / \mathrm{mol}$ über dem globalen Minimum 14 verschiedene Dimerstrukturen gefunden werden.

Für den Vergleich der harmonisch vorhergesagten Dimerübergänge mit den gemessenen Spektren der Jetexpansionen in Abbildung 4.2 wurden die Wellenzahlen mit dem Faktor 0.9569 skaliert, der sich aus dem gemittelten Verhältnis der theoretischen und experimentellen Wellenzahlen der beiden $\mathrm{O}-\mathrm{H}$ Streckschwingungen des Konformer I von Ethylenglykol ergibt. Außerdem wurden die vorhergesagten Dimerbanden als Lorentzfunktionen mit $6 \mathrm{~cm}^{-1}$ Halbwertsbreite dar- 


\section{4. $O-H$ Streckschwingungen}

gestellt und so skaliert, dass sie einer Konzentration der Dimere von je 10\% des Monomers in Spektrum b der Abbildung 4.2 entsprechen. Die unskalierten Werte der harmonischen Vorhersage sind in Tabelle A.12 im Anhang gegeben. Der Vergleich in Abbildung 4.2 zeigt, dass sich anhand der einzelnen experimentellen Dimerbande keine Zuordnung der verschiedenen Dimerstrukturen treffen lässt, da jeweils eine besonders intensive Bande für die Dimere vorhergesagt wird. Das einzige Maß zur Unterscheidung der Strukturen wäre die Rotverschiebung ihrer Übergänge, diese wird jedoch sehr ähnlich vorhergesagt und lässt keine klare Identifizierung zu.

Der einzige Hinweis auf das bevorzugte Dimer des Ethylenglykol bleibt damit der deutliche energetische Vorsprung von 4H auf B3LYP-D3/6-311+G(2d,p) Niveau. Um das Dimerspektrum genauer zu untersuchen bieten sich Messungen am Popcorn-Jet an, um durch die beheizte Düse eine höhere Dimerkonzentration zu erreichen. Aber auch Ramanmessungen beispielsweise am Curry-Jet wären hilfreich, da dort nicht nur die IR-verbotenen Übergänge von $4 \mathrm{H}$ oder $2 \mathrm{H}$ sichtbar gemacht werden könnten, sondern auf Grund der höheren Sensitivität der Messungen auch eventuelle freie $\mathrm{O}-\mathrm{H}$ Gruppen im Dimer nachweisbar wären. Die Aufspaltung zwischen den IR-aktiven und Raman-aktiven Moden des Dimers sollte bei der Zuordnung der Strukturen zum Spektrum helfen. Davon abgesehen wäre die Untersuchung des Ethylenglykolmonomers im Ferninfrarotbereich interessant. Dort könnte die Fragestellung untersucht werden, ob die Anregung der $\mathrm{O}-\mathrm{H}$ Torsionen zu einer Vergrößerung der Tunnelaufspaltung gegenüber dem Grundzustand ${ }^{77}$ führt. Auch dazu würde sich eine Kombination aus Infrarot- und Ramanspektroskopie anbieten.

\subsubsection{Propylenglykol}

Durch die Methylierung eines Kohlenstoffatoms von Ethylenglykol wird ein stereogenes Zentrum eingeführt - es ergibt sich Propylenglykol (Propan-1,2-diol). Durch die zusätzliche Methylgruppe entsteht auch eine größere Vielfalt an möglichen Monomerkonformationen. In Abbildung 4.4 sind die sechs stabilsten Konformationen von Propylenglykol dargestellt, die sich auf B3LYP-D3/6-311+G(2d,p)-Niveau ergeben. Die Nomenklatur durch die Ziffern I bis VI zu den Konformeren richtet sich nach Referenz 106. In der Gasphase konnten zunächst mittels Mikrowellenspektroskopie die Konformere I und II identifiziert werden. ${ }^{107}$ Dabei wurde für letzteres ein energetischer Vorteil von 2.4(4) kJ/mol bestimmt. ${ }^{107}$ Die Nutzung von Überschallexpansionen zur Kühlung der Moleküle ermöglichte die Zuordnung des Konformers III im Rotationsspektrum und seine Identifizierung als stabilstes Konformer. ${ }^{108}$ Schließlich konnten mittels Mikrowellenspektroskopie sogar sieben Konformere von Propylenglykol in Überschallexpansionen nachgewiesen werden. ${ }^{106}$ Sie weisen alle einen gauche Diederwinkel zwischen den beiden $\mathrm{C}-\mathrm{O}$ Gruppen auf.

Durch Messung des IR-Spektrums von Propylenglykol konnten in Lösung gebundene und freie $\mathrm{O}-\mathrm{H}$ Gruppen nachgewiesen werden. ${ }^{109}$ Wie auch im Falle von Ethylenglykol kann Propylenglykol in Ar-Matrizen durch Bestrahlung mit Infrarotlicht in Konformere umgewandelt werden, die einen trans Diederwinkel zwischen den C-O Gruppen aufweisen. ${ }^{110}$

Die in Abbildung 4.4 dargestellten Monomerkonformere II, III, V und VI unterscheiden sich nur in der Ausrichtung der Hydroxylgruppen, während die terminale $\mathrm{C}-\mathrm{O}$ Bindung trans zur Methylgruppe steht. In den Konformeren I und IV steht die terminale $\mathrm{C}-\mathrm{O}$ Bindung dagegen gauche 


\subsection{Fundamentalübergänge}

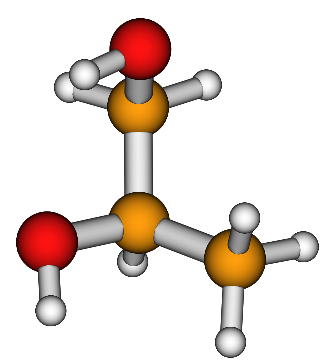

2.1

I

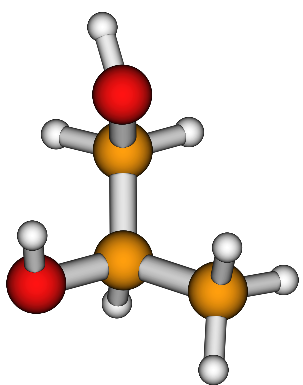

4.0

IV

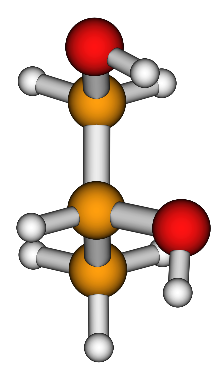

0.1

II

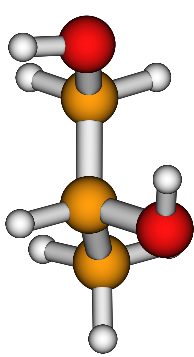

0.8

$\mathrm{V}$

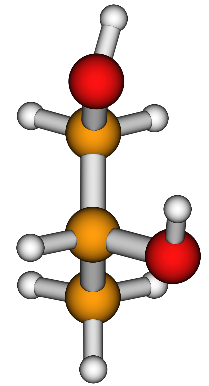

0.0

III

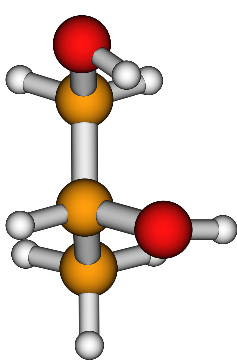

1.3

VI

Abbildung 4.4.: Berechnete Strukturen (B3LYP-D3/6-311+G(2d,p)) der sechs günstigsten Konformere von $(R)$-Propylenglykol. Die relativen Energien unter Berücksichtigung der harmonischen Nullpunktsenergien sind in $\mathrm{kJ} / \mathrm{mol}$ gegeben.

zur Methylgruppe. Ihre relativen Energien auf dem hier verwendeten B3LYP-D3/6-311+G(2d,p)Niveau sind zusammen mit den Werten auf MP2/aug-cc-pVTZ-Niveau ${ }^{106}$ in Tabelle 4.2 zusammengefasst. Auf beiden Rechenniveaus sind III, II und V die drei stabilsten Konformere.

In Abbildung 4.5 sind die $\mathrm{O}-\mathrm{H}-\mathrm{Streckschwingungsspektren} \mathrm{der} \mathrm{Überschallexpansionen} \mathrm{von}$ Propylenglykol in Helium am Filet-Jet im Bereich der Monomere gezeigt. Das Spektrum a wurde zusammen mit Daniel Falkowski im Zuge seiner Bachelorarbeit aufgenommen ${ }^{71}$ (ebenfalls als Spektrum a in Abbildung 4.6 zu finden). Es zeigen sich in Abbildung 4.5 fünf Monomersignale M1 bis M5 bei 3689, 3673, 3657, 3626 und $3614 \mathrm{~cm}^{-1}$. Um sie den Monomeren zuzuordnen wurden anharmonische Rechnungen auf B3LYP-D3/6-311+G(2d,p)-Niveau durchgeführt. Dazu wurde zur Optimierung das „VeryTight“-Kriterium verwendet und die Rechnungen mit dem „Superfine“-Gitter vom Programm Gaussian 09 Rev. D01 ${ }^{68}$ durchgeführt. Ein Faktor zur Skalierung der anharmonischen Wellenzahlen lässt sich aus dem experimentellen Wellenzahlen für das Ethylenglykolkonformer I (siehe vorhergehenden Abschnitt 4.1.2) und der anharmonischen Vorhersage auf dem hier benannten Rechenniveau (s. Tabelle A.12 im Anhang) gewinnen. Dieser Faktor beträgt für das Mittel der beiden $\mathrm{O}-\mathrm{H}$ Streckschwingungen des stabilsten Ethylenglykolmonomer 1.005, und wurde mit den vorhergesagten Wellenzahlen der Propylenglykolmonomere multipliziert. Die resultierende Zuordnung der Monomerkonformationen zu den experimentellen 


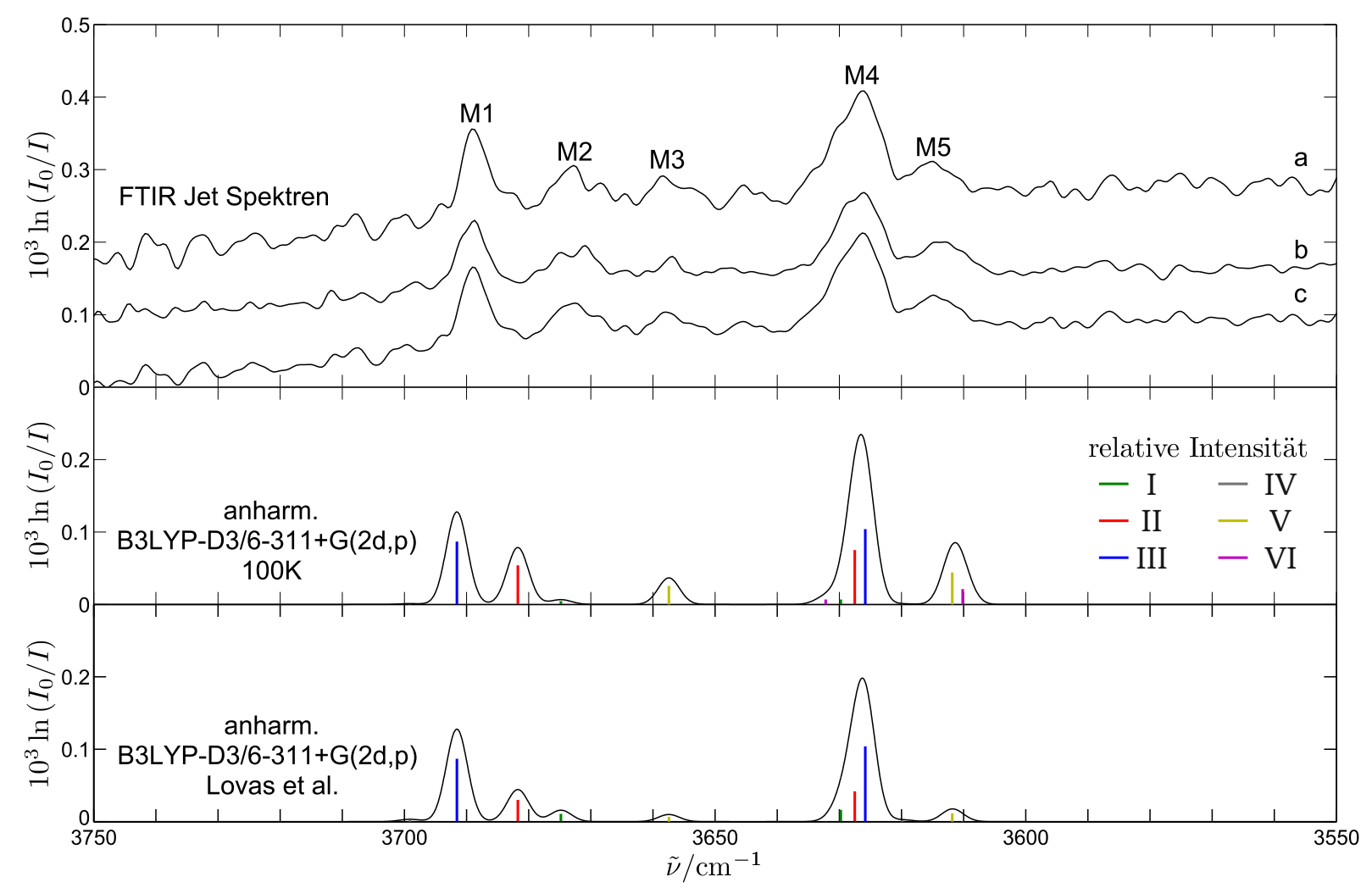

Abbildung 4.5.: Vergleich der FTIR-Spektren von Propylenglykol in den Expansionen des $\mathrm{Fi}$ let-Jet mit anharmonischen Vorhersagen. Oberes Fenster: experimentelle Spektren $\mathrm{a}-0.007 \%$ in 1.3 bar Helium (150 Pulse), b - $0.007 \%$ in 0.7 bar Helium (400 Pulse) und c - Mittelwertspektrum aus a und b. Mittleres und unteres Fenster: Anharmonische Vorhersagen der Monomere auf B3LYP-D3/6$311+\mathrm{G}(2 \mathrm{~d}, \mathrm{p})-\mathrm{Niveau}$, deren Wellenzahlen mit dem Faktor 1.005 skaliert wurden. Die relativen Intensitäten der Monomere sind als farbige Balken dargestellt, das resultierende Spektrum aus allen Monomerübergängen ist schwarz und basiert auf Gaussfunktionen mit $4 \mathrm{~cm}^{-1}$ Halbwertsbreite. Die Konzentration von Monomer III wurde aus der Bande M1 abgeschätzt und dient als Grundlage für die Skalierung der berechneten Monomerspektren. Im mittleren Fenster entspricht die Häufigkeit der Monomere einer Boltzmannverteilung für eine Konformationstemperatur von $100 \mathrm{~K}$ und im unteren Fenster der in Referenz 106 beschriebenen experimentellen Verteilung in einer He/Ne-Expansion.

Signalen ist in Tabelle 4.2 gegeben, die unskalierten Ergebnisse der anharmonischen Vorhersage in Tabelle A.12 im Anhang. Die zwei intensivsten Banden im Spektrum M1 und M4 können so teilweise dem stabilsten Konformer III zugeordnet werden. In M4 überlagern wahrscheinlich die Signale mehrerer Konformere.

Durch die Kühlung in Überschallexpansionen relaxieren die Moleküle in die stabilsten Kon- 


\subsection{Fundamentalübergänge}

Tabelle 4.2.: Die sechs stabilsten Konformere von Propylenglykol: berechnete relative Energien $\Delta E_{0}$ inklusive harmonischer Nullpunktskorrektur, Zuordnung zu den gemessenen $\mathrm{O}-\mathrm{H}$ Streckschwingungsbanden am Filet-Jet, sowie aus den anharmonischen Intensitäten abgeschätzte relative Besetzung der Konformere in den He-Expansionen des Filet-Jet. Zum Vergleich ist die durch Mikrowellenspektroskopie ermittelte relative Besetzung in He/Ne-Expansionen aus einer Lochdüse ${ }^{106}$ gegeben.

\begin{tabular}{ccccc}
\hline Konformer & $\Delta E_{0} / \mathrm{kJ} / \mathrm{mol}^{a}$ & $\begin{array}{c}\text { Zuordnung } \\
\text { FTIR Spektrum }\end{array}$ & $\begin{array}{c}\text { rel.Häufigkeit } \\
\text { Filet-Jet }\end{array}$ & $\begin{array}{c}\text { rel.Häufigkeit } \\
\text { He/Ne-Expansion }\end{array}$ \\
\hline I & $2.1 / 2.5$ & M2/M4 & $0.7-0.8^{c}$ & 0.18 \\
II & $0.1 / 0.9$ & M2/M4 & 1.0 & 0.44 \\
III & $0.0 / 0.0$ & M1/M4 & - & 1.0 \\
IV & $4.0 / 4.2$ & - & $0.2-0.3$ & 0.023 \\
V & $0.8 / 1.4$ & M3/M5 & $0.1-0.5$ & 0.11 \\
VI & $1.3 / 2.8$ & M4/M5 & & 0.056 \\
\hline
\end{tabular}

${ }^{a}$ B3LYP-D3/6-311+G(2d,p) / MP2/aug-cc-pVTZ ${ }^{106}$

${ }^{b}$ MW Spektrum Ref. 106

${ }^{c}$ Konformer I+II

formationen. In Referenz 106 sind die Häufigkeiten der einzelnen Monomerkonformationen in einer Expansion (1bar Puffergas: 20\% Helium, 80\% Neon) von Propylenglykol aus einer auf $60^{\circ} \mathrm{C}$ geheizten $1 \mathrm{~mm}$ Lochdüse beschrieben. Es dominieren vier Konformere: III, II, I und V, deren relative Häufigkeit in dieser Reihenfolge abnimmt. In Abbildung 4.4 ist im unteren Fenster ein simuliertes Spektrum auf Grundlage der skalierten anharmonischen Wellenzahlen und Intensitäten für die in Referenz 106 beschriebene Monomerverteilung dargestellt. Alternativ ist das vorhergesagte anharmonische Spektrum einer Boltzmannverteilung der Monomere bei $100 \mathrm{~K}$ Konformationstemperatur auf Grundlage der relativen Energieunterschiede auf B3LYP-D3/6$311+\mathrm{G}(2 \mathrm{~d}, \mathrm{p})$-Niveau im mittleren Fenster aufgetragen.

In beiden simulierten Spektren ist Konformer I mit nur geringer Population von 0.08 bis 0.18 relativ zum stabilsten Konformer III vorhanden, was etwa 3 bis 10\% aller Monomere entspricht. Jedoch könnte es sein, dass in der Heliumexpansion am Filet-Jet das Konformer I viel häufiger vorliegt, da die Umwandlung in die stabileren Konformere eine Änderung des $\mathrm{O}-\mathrm{C}-\mathrm{C}-\mathrm{C}$ Diederwinkels erfordert und damit kinetisch stärker gehindert sein könnte - im Vergleich zur Umwandlung der verschiedenen $\mathrm{O}-\mathrm{H}$ Torsionsisomere ineinander. Die Population von Konformer I und der anderen Monomere mit gauche Diederwinkel dieser Atome liegt bei Raumtemperatur bei ungefähr 20\%. ${ }^{106}$ Der Anteil von I könnte experimentell also deutlich höher liegen und einen signifikanten Anteil an der Intensität des Signals M2 ausmachen. Die Signale M3 und M5 erscheinen in den Spektren des Filet-Jet etwas intensiver als im simulierten Spektrum nach Referenz 106 und ähneln in ihrer Intensität eher dem simulierten Spektrum für $100 \mathrm{~K}$. Dies spricht dafür, dass die zu Grunde liegenden Konformere V und VI in den Expansionen des Filet-Jet weniger stark relaxieren als in der Expansion in Referenz 106. Der hohe Neonanteil im Puffergas könnte dort zu der für diese beiden Konformere beobachteten besonders effizienten Relaxation 


\section{4. $O-H$ Streckschwingungen}

geführt haben.

Die Verteilung der Monomerkonformere in der Expansion am Filet-Jet lässt sich auch mit Hilfe der vorhergesagten anharmonischen Intensitäten abschätzen. Diese abgeschätzten Besetzungen der Konformationen sind in Tabelle 4.2 zusammen mit der Verteilung aus Referenz 106 dargestellt. Allgemein ergibt sich am Filet-Jet eine etwas häufigere Besetzung der weniger stabilen Konformere gegenüber dem stabilsten Monomer III, was auf eine stärkere Kühlung in der Expansion mit hohem Neonanteil ${ }^{106}$ hindeutet. Außerdem liegt möglicherweise eine höhere Besetzung des Konformer VI vor, sodass sich in den Spektren des Filet-Jet bis zu fünf signifikant besetzte Monomere zeigen könnten. Allerdings ist die Abschätzung der Verteilung in den Expansionen des Filet-Jet durch die Ungenauigkeit der angewendeten Signalintegration und vorhergesagten anharmonischen Intensitäten sowie der Überlappung der Monomerbanden sicherlich mit einem deutlichen Fehler behaftet. Dieser wird bei der Abschätzung der Besetzung von Monomer VI auf Grund der Überlappung mit Monomer V im Signal M5 besonders groß sein.

Wie Ethylenglykol weist auch Propylenglykol einen äußerst geringen Dampfdruck auf, sodass in den Überschallexpansionen des Filet-Jet nur sehr schwache Dimersignale zu beobachten sind. Deswegen wurden Spektren der Expansionen von Propylenglykol am Popcorn-Jet aufgenommen, um die höhere Konzentration der Dimere durch die Erhitzung der Substanz zu nutzen. Diese Resultate beider Experimente im O-H Streckschwingungsbereich sind in Abbildung 4.6 dargestellt. In den Spektren der enantiomerenreinen Substanz $(a, b)$ sind drei Clusterbanden zu finden, die auf homochirale Dimere zurückzuführen sind, da sie sowohl in den Popcorn- als auch Filet-Jetspektren erscheinen und für letztere Signale größerer Cluster ausgeschlossen werden können. Die Dimerbanden liegen bei 3506, 3498 und $3492 \mathrm{~cm}^{-1}$ recht nah beieinander. Bei der Untersuchung des racemischen Gemisches (c, d, e) zeigt sich eine Erhöhung der Intensität im mittleren Bereich der vorgenannten Signale. Das Spektrum der heterochiralen Dimere kann unter Annahme statistischer Bildung der Dimere in den Expansionen aus den Spektren der enantiomerenreinen Substanz und des Racemats, die unter identischen experimentellen Bedingungen erzeugt wurden, synthetisiert werden. Dazu wurde Spektrum d mit 2 multipliziert und Spektrum b davon subtrahiert. Es resultiert ein Differenzspektrum mit einer einzelnen Bande bei $3500 \mathrm{~cm}^{-1}$ der heterochiralen Dimere. Das Spektrum e des Racemats wurde bei der höchsten Substanzkonzentration aufgenommen. Dort zeigen sich von 3470 bis $3200 \mathrm{~cm}^{-1}$ zwei breite Absorptionsbereiche die auf die Signale größerer Cluster zurückzuführen sind.

In Abbildung 4.7 sind die acht stabilsten Dimere gezeigt (von etwa 200 berechneten Strukturen), die auf B3LYP-D3/6-311+G(2d,p)-Niveau für Propylenglykol gefunden wurden. Ihre Benennung basiert auf der für Ethylenglykol im vorhergehenden Abschnitt 4.1.2 verwendeten Nomenklatur. Sie wird mit den Präfixen RR und RS erweitert um homochirale und heterochirale Strukturen zu bezeichnen. Die Suffixe a und b kennzeichnen ähnliche Strukturen, die sich nur in der Ausrichtung der Methylgruppen unterscheiden. Die 4H (vier cyclische intermolekulare Wasserstoffbrücken) und 2H (zwei inter- und zwei intramolekulare Wasserstoffbrücken) Strukturen von Propylenglykol weisen die gleichen Wasserstoffbrückengeometrien und Ausrichtung der $\mathrm{O}-\mathrm{C}-\mathrm{C}-\mathrm{O}$ Gerüste wie die entsprechenden Ethylenglykoldimere auf und unterscheiden sich von ihnen nur durch die zwei zusätzlichen Methylgruppen. Die einzige Ausnahme dafür ist RR2H'. Das angehängte ' zeigt für RR2H' und RS3H' an, dass es für diese Strukturen keine Entsprechung bei den vorgestellten Strukturen der Ethylenglykoldimere in Abschnitt 4.1.2 gibt. 


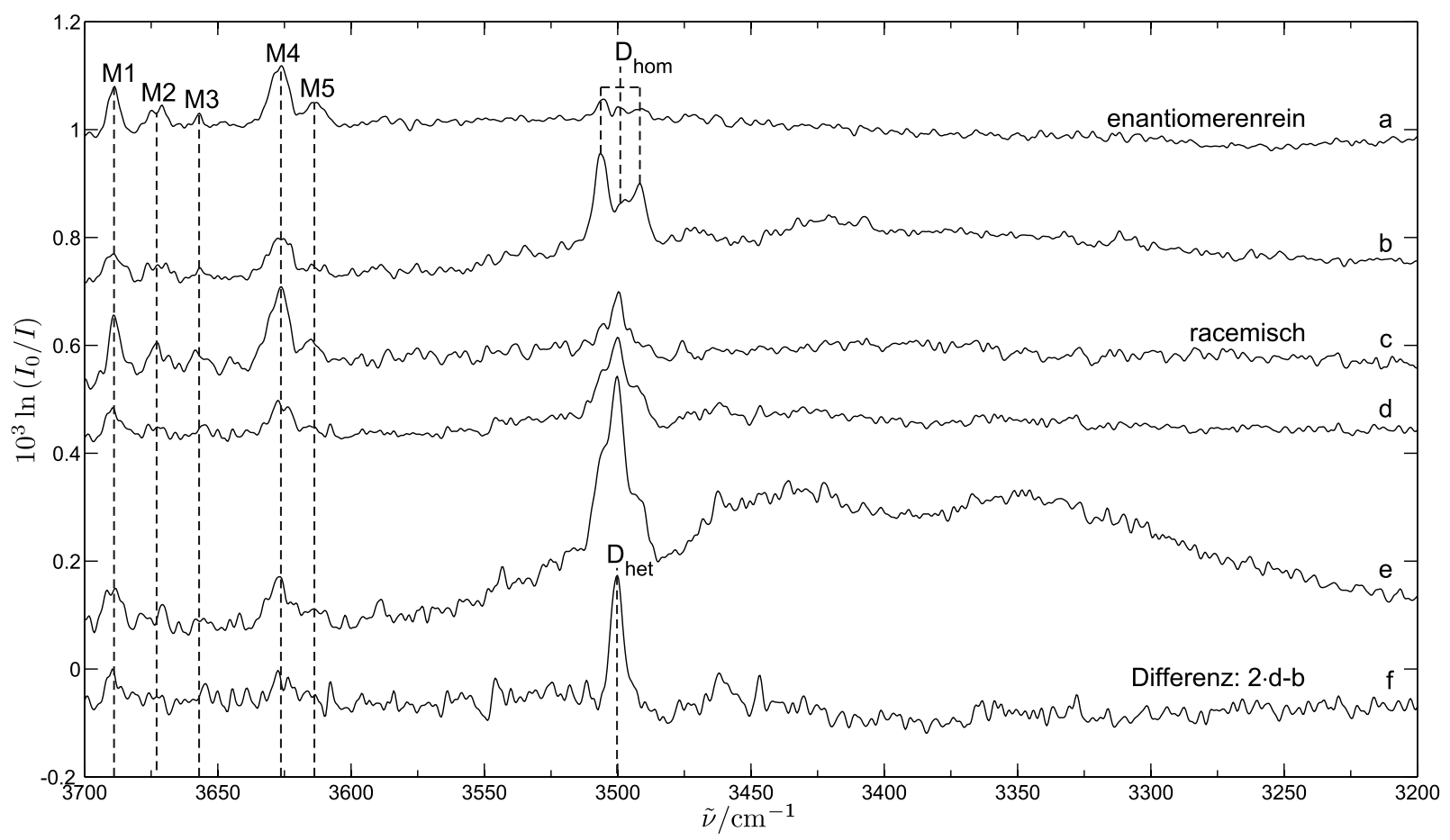

Abbildung 4.6.: FTIR Spektren von Propylenglykol in Helium-Überschallexpansionen am $F i$ let-Jet und am Popcorn-Jet. Enantiomerenreines Propylenglykol: a - FiletJetspektrum, $0.007 \%$ in 0.7 bar Helium (400 Pulse) und b - Popcorn-Jetspektrum in 1.0 bar Helium (Substanz $60^{\circ} \mathrm{C}$, Düse $80^{\circ} \mathrm{C}$, 250 Pulse). Racemisches Propylenglykol: c - Filet-Jetspektrum, $0.01 \%$ in 1.3 bar Helium (150 Pulse), d Popcorn-Jetspektrum in 1.0 bar Helium (Substanz $60^{\circ} \mathrm{C}$, Düse $80^{\circ} \mathrm{C}, 250$ Pulse) und e - Popcorn-Jetspektrum in 1.0 bar Helium (Substanz $70^{\circ} \mathrm{C}$, Düse $90^{\circ} \mathrm{C}$, 140 Pulse). Spektrum $\mathrm{f}$ ist ein synthetisches Spektrum der heterochiralen Dimere und wurde durch Multiplikation von Spektrum d mit 2 und anschließender Subtraktion von Spektrum b erhalten.

Das stabilste gefundene Dimer ist das heterochirale RS4Ha, gefolgt von zwei homochiralen 4H Strukturen. Das zweitstabilste heterochirale Dimer ist RS2H und gegenüber dem stabilsten um $5.5 \mathrm{~kJ} / \mathrm{mol}$ benachteiligt. Es folgen RS4Hb und RS3H', die 6.9 bzw. $7.1 \mathrm{~kJ} / \mathrm{mol}$ weniger stabil als RS4Ha sind. Im Falle der homochiralen Dimere sind die energetischen Abstände zur stabilsten Struktur deutlich kleiner. Auf RR4Ha folgt RR4Hb mit nur $0.8 \mathrm{~kJ} / \mathrm{mol}$ geringerer Dissoziationsenergie. RR2H' ist um 2.2 und RR2H um $2.7 \mathrm{~kJ} / \mathrm{mol}$ gegenüber dem stabilsten homochiralen Dimer RR4Ha benachteiligt. In der Literatur findet sich bisher nur die Untersuchung der Dimere von Propylenglykol mit Hilfe von Kraftfeldmethoden, ${ }^{111}$ die damit gefundenen stabilsten Strukturen stimmen aber nicht mit den hier vorgestellten Dimeren überein.

Für den Vergleich der harmonischen Schwingungsvorhersage mit den Spektren des Filet-Jet in Abbildung 4.8 wurden die Wellenzahlen vom B3LYP-D3/6-311+G(2d,p)-Niveau mit dem Faktor 0.9569 multipliziert, der auch für die Dimerrechnungen von Ethylenglykol verwendet wurde (s. 


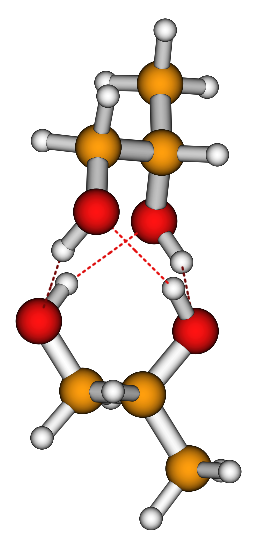

50.6

$\mathrm{RS} 4 \mathrm{Ha}$

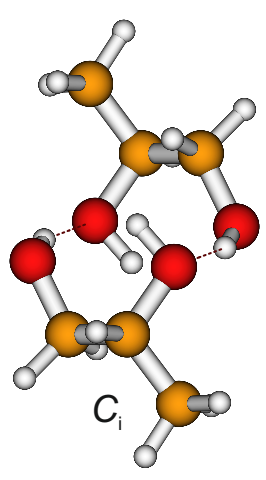

45.1

$\mathrm{RS} 2 \mathrm{H}$

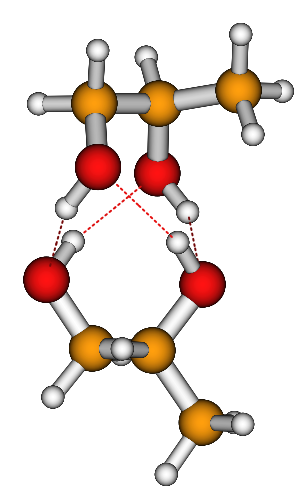

47.4

RR4Ha

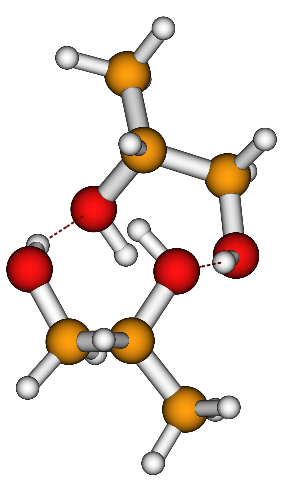

44.7

$\mathrm{RR} 2 \mathrm{H}$

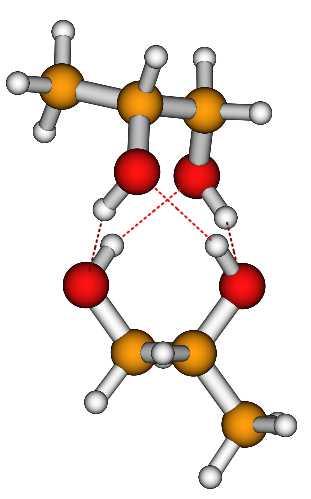

46.6

$\mathrm{RR} 4 \mathrm{Hb}$

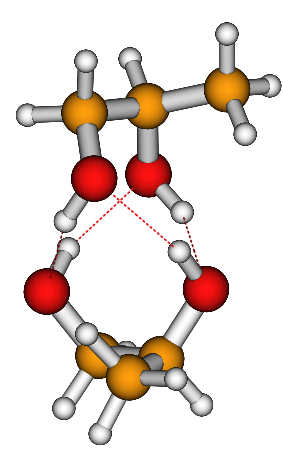

43.7

$\mathrm{RS} 4 \mathrm{Hb}$

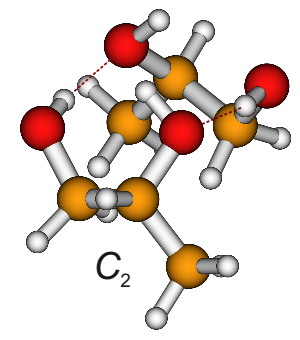

45.2

$\mathrm{RR} 2 \mathrm{H}^{*}$

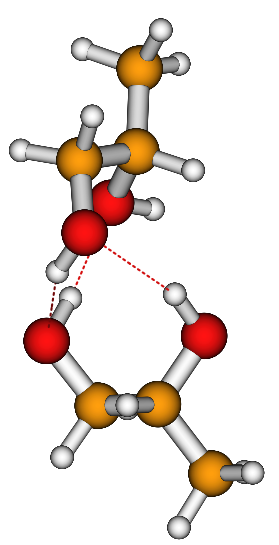

43.5

$\mathrm{RS} 3 \mathrm{H}^{\prime}$

Abbildung 4.7.: Strukturen der acht stabilsten Dimere von Propylenglykol auf B3LYP-D3/6$311+\mathrm{G}(2 \mathrm{~d}, \mathrm{p})$ Niveau und Dissoziationsenergien $D_{0}$ in $\mathrm{kJ} / \mathrm{mol}$ unter Berücksichtigung der harmonischen Nullpunktsenergien.

Abschnitt 4.1.2). Die vorhergesagten Intensitäten wurden für jedes Dimer auf eine ungefähre Konzentration von $10 \%$ gegenüber den Monomeren skaliert. Die Dimerbanden wurden dann als Lorentzfunktionen mit $6 \mathrm{~cm}^{-1}$ Halbwertsbreite aufgetragen.

Auf Grund der einzelnen experimentellen Bande ist das synthetische Spektrum der heterochiralen Dimere etwas einfacher zu diskutieren. Die simpelste Erklärung für die Bande ist, dass nur die stabilste heterochirale Dimerstruktur RS4Ha, für die eine Bande mit hoher IR-Intensität vorhergesagt wird, im Spektrum zu beobachten ist. Dafür spricht der berechnete deutliche Energievorsprung dieser Struktur. Eine sichere Zuordnung dieser Struktur lässt sich aber erst treffen, wenn auch die anderen Banden dieses Dimers experimentell beobachtet werden können, da auch für RS4Hb und RS2H je nur eine intensive Bande vorhergesagt wird. Möglich wäre auch eine spektrale Überlappung zwischen RS4Ha und RS2H. Ausschließen lässt sich dagegen ein signifikanter Anteil von RS3H' in der Überschallexpansion, da für diese Struktur zwei etwa gleich 


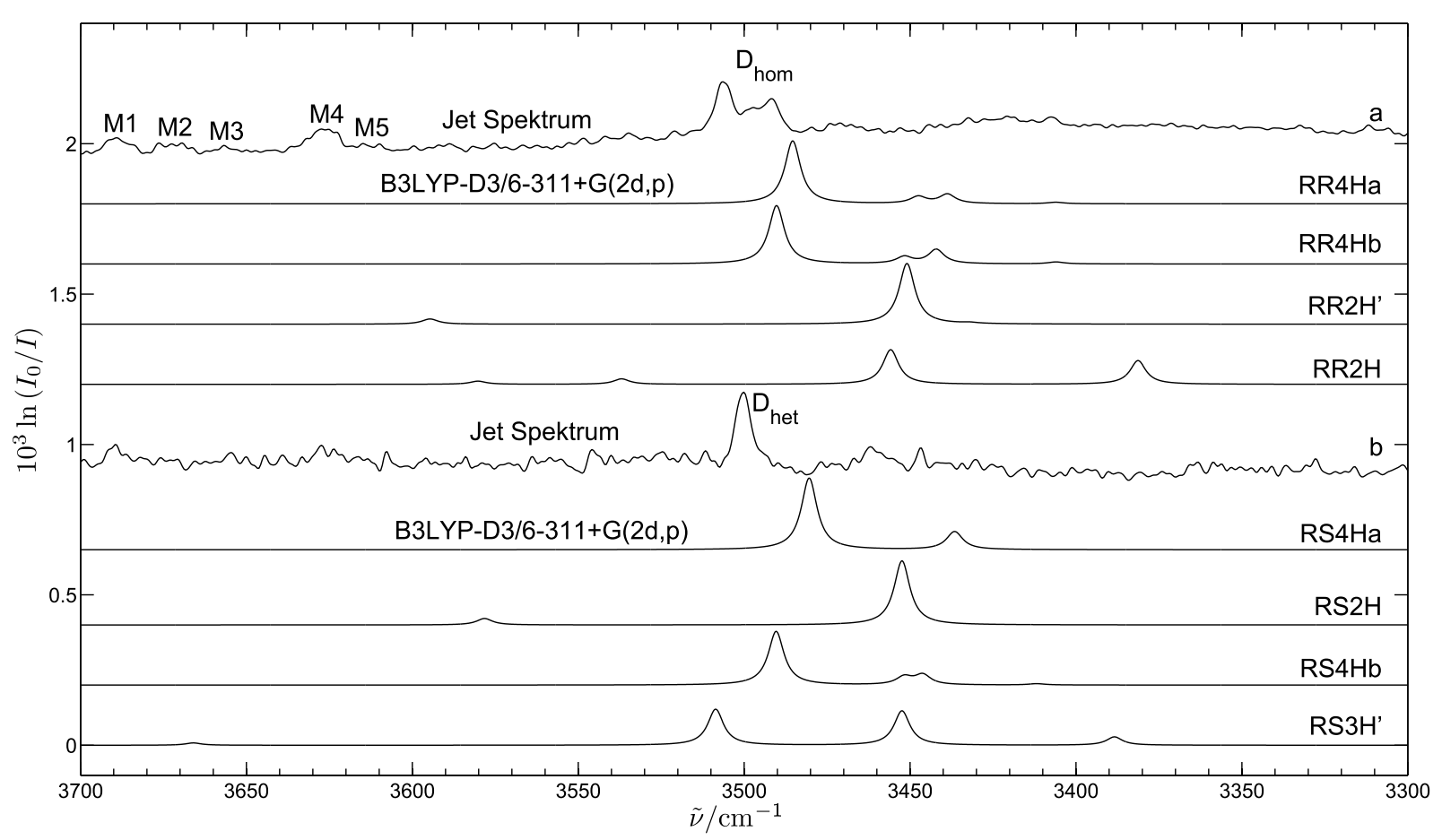

Abbildung 4.8.: Vergleich der FTIR-Spektren von Propylenglykol in Jetexpansionen des Popcorn-Jet mit berechneten Spektren der Dimere. Die experimentellen Spektren zeigen homochirale Dimerbanden (a) oder heterochirale Dimerbanden (b). Sie entsprechen den Spektren $b$ und $f$ aus Abbildung 4.6. Für die harmonischen Vorhersagen auf B3LYP-D3/6-311+G(2d,p)-Niveau wurden die Wellenzahlen mit dem Faktor 0.9569 und die Intensitäten jeweils auf eine Dimerkonzentration von ungefähr 10\% der Monomerkonzentration skaliert.

intensive Banden vorhergesagt werden.

Im Spektrum der homochiralen Dimere wurden drei Signale beobachtet. Die einfachste Erklärung dafür wäre das Vorliegen mehrerer Dimerstrukturen. Da im Falle der homochiralen Dimere die berechneten energetischen Abstände deutlich geringer sind, wäre auch dieser Befund mit den theoretischen Ergebnissen kompatibel. Die drei stabilsten Dimere RR4Ha, RR4Hb und RR2H' sollten je nur eine intensive Bande aufweisen, somit wäre die Zuordnung aller drei Strukturen im Spektrum naheliegend. Die Dimerbande bei der höchsten Wellenzahl von $3506 \mathrm{~cm}^{-1}$ zeigt auch die höchste Intensität. Dies deutet darauf hin, dass sie auf das stabilste Dimer und damit wahrscheinlich RR4Ha zurückgeht. RR4Ha würde auch mit $-21 \mathrm{~cm}^{-1}$ eine ähnliche Abweichung zwischen skalierter Vorhersage und dieser experimentellen Bande bei $3506 \mathrm{~cm}^{-1}$ zeigen wie das heterochirale Dimer RS4H zur heterochiralen Dimerbande $\left(-20 \mathrm{~cm}^{-1}\right)$ und das stabilste Ethylenglykoldimer zur entsprechenden experimentellen Bande $\left(-23 \mathrm{~cm}^{-1}\right)$. Beide Dimere weisen dasselbe Strukturmotiv wie RR4Ha von vier Wasserstoffbrücken auf und sollten somit in der harmonischen Vorhersage auf ähnliche Weise vom Experiment abweichen. Auch RR4Hb zeigt dieses Bindungsmotiv auf, würde aber bei einer Zuordnung zu der homochiralen Dimerbande 


\section{4. $O-H$ Streckschwingungen}

bei $3506 \mathrm{~cm}^{-1}$ nur eine Abweichung der skalierten Wellenzahl von $-16 \mathrm{~cm}^{-1}$ aufweisen, die im Falle der anderen Dimerbanden noch geringer ausfallen würde. Dies ist ein Hinweis darauf, dass RR4Hb möglicherweise nicht in den Überschallexpansionen des Filet-Jet vorliegt, wenn die Annahme, dass die stabilsten 4H Strukturen von Propylenglykol und Ethylenglykol tatsächlich beobachtet werden, zutrifft. RR2H' zeigt dagegen ein anderes Bindungsmotiv und kann also trotz der größeren Abweichung der skalierten harmonischen Wellenzahlen in der Überschallexpansion vorliegen. Nahezu ausschließen lässt sich RR2H, da für diese Struktur eine weitere intensive Bande mit größerer Rotverschiebung vorhergesagt wird, was im Experiment nicht gefunden wurde. Auch im Falle der homochiralen Dimere steht die Beobachtung der schwächeren Dimerbanden noch aus, um die Zuordnung der Dimerstrukturen abzusichern. Dazu würde sich wie auch im Falle der heterochiralen Dimere beispielsweise Überschallexpansionen aus der neuen Hochtemperaturdüse des Popcorn-Jet anbieten, ${ }^{65}$ da sich mit dieser bei höheren Substanzkonzentrationen die Bildung größerer Cluster unterdrücken lässt. Aber auch die Anwendung der Ramanspektroskopie würde die Beobachtung der schwächeren Dimerbanden und IR-verbotenen Übergänge ermöglichen. Interessant wäre auch die Untersuchung der homochiralen Dimere in Überschallexpansionen unter Zumischung von Argon, da sich damit möglicherweise die Relaxation zum stabilsten Dimer verbessern und somit die theoretisch vorhergesagte Energiereihenfolge überprüfen ließe.

Zum Abschluss lassen sich noch Überlegungen anstellen, woher die energetischen Unterschiede der 4H Strukturen des Propylenglykoldimers rühren. Da sie alle das gleiche Wasserstoffbrückenmotiv aufweisen, sollten sich die Dissoziationsenergien nicht auf Grund unterschiedlicher zwischenmolekularer Anziehung unterscheiden. Auch die Methylgruppen können nicht zu sterischer Hinderung zwischen den Molekülen führen, da sie jeweils zum anderen Moleküle abgewandt liegen. Der energetische Effekt sollte daher ein intramolekularer sein. Die Hydroxygruppe am stereogenen Zentrum des Propylenglykols kann in den 4H Strukturen entweder einen Diederwinkel von etwa $40^{\circ}$ oder etwa $150^{\circ}$ zu der Methylgruppe des Moleküls einnehmen. Letzterer scheint energetisch günstiger zu sein, da im global stabilsten Dimer RS4Ha in beiden Molekülen dieser Diederwinkel vorliegt. In den homochiralen Dimerstrukturen RR4Ha und RR4Hb wird je eine Hydroxylgruppe in den weniger bevorzugten Diederwinkel zur Methylgruppe gezwungen, was zu einem Energienachteil von etwa $3.5 \mathrm{~kJ} / \mathrm{mol}$ führt. In RS4Hb schließlich stehen in beiden Molekülen die Hydroxylgruppen an den stereogenen Zentren im ungünstigeren Diederwinkel von etwa $40^{\circ} \mathrm{zu}$ den Methylgruppen, resultierend in einem Energienachteil von etwa $7 \mathrm{~kJ} / \mathrm{mol}$. Dies bringt RS4Hb in energetische Konkurrenz zu den $2 \mathrm{H}$ Strukturen, die etwa 5 bis 6 $\mathrm{kJ} / \mathrm{mol}$ gegenüber dem globalen Minimum vorhergesagt werden.

Dieser Befund führt zu einer interessanten Voraussage für die Dimere von Butan-2,3-diol, das sich durch weitere Methylierung aus Propylenglykol ergibt. Durch seine zwei stereogenen Zentren kann Butan-2,3-diol als chirales Molekül in $R, R$ oder $S, S$ Form vorliegen oder als achirales Molekül in $R, S$ (meso) Form, was bereits zu Effekten von intramolekularer Chiralitätserkennung führt. ${ }^{112}$ Wenn die Dimere von Butan-2,3-diol 4H Strukturen bilden, liegen dort vier der Wechselwirkungen zwischen Hydroxyl- und Methylgruppen vor. Im Falle der chiralen Form ließen sich im homochiralen Dimer zwei ungünstige Diederwinkel in einem der beiden Moleküle erzwingen, sodass in diesem Falle die 2H Strukturen stabiler sein könnten. Im Falle des heterochiralen Dimers könnte diese ungünstige Wechselwirkung jedoch vermieden werden. Hinzu kommt 
aber auch die intramolekulare Abstoßung der Methylgruppen, die im Falle des chiralen Moleküls vermieden werden kann, auch wenn beide $\mathrm{C}-\mathrm{O}$ Bindungen gauche zueinander stehen. ${ }^{112}$ In der meso Form des Moleküls lässt sich die Abstoßung der beiden Methylgruppen bei gauche Anordnung der $\mathrm{C}-\mathrm{O}$ Bindungen nicht vermeiden. ${ }^{112}$ Im Dimer mit 4H Struktur von meso-Butan-2,3diol würde je ein ungünstiger Diederwinkel in den zwei Molekülen erzwungen, was es ebenfalls in Konkurrenz mit den 2H Strukturen bringen sollte. Diese Konkurrenzen zwischen $4 \mathrm{H}$ und $2 \mathrm{H}$ Strukturen könnten die Dimere von Butan-2,3-diol zu interessanten Testfällen für quantenchemische Energievorhersagen machen, da $4 \mathrm{H}$ und $2 \mathrm{H}$ Strukturen energetisch nah beieinanderliegen und spektroskopisch in Überschallexpansionen gut unterscheidbar sein sollten. Es könnten aber auch andere Strukturmotive der Dimere vorliegen, die aus der zunehmenden Komplexität des Systems resultieren. 


\section{4. $\mathrm{O}-\mathrm{H}$ Streckschwingungen}

\subsection{Obertöne}

Nachdem Trifluorethanol als erster Alkohol durch Obertonspektroskopie auf anharmonische Effekte in der $\mathrm{O}-\mathrm{H}-$ Streckschwingung des Dimers in Jetexpansionen charakterisiert wurde, ${ }^{43,47}$ soll hier die Untersuchung auf weitere Alkohole ausgedehnt werden. Zusammen mit Kim Papendorf wurden das ebenfalls fluorierte Hexafluorisopropanol (HFIP), ${ }^{7}$ sowie die grundlegenden Substanzen Methanol $(\mathrm{MeOH})$ und Ethanol $(\mathrm{EtOH})^{11}$ als Systeme gewählt und experimentell analysiert. ${ }^{113}$ Außerdem wurde das Dimer von tert-Butylalkohol $(t-\mathrm{BuOH})$ untersucht. ${ }^{11}$ Zuvor wurden in der Literatur für die Gasphase vor allem die Obertonspektren von Alkoholmonomeren publiziert, ${ }^{85,114-116}$ wobei besonders Methanol oft untersucht wurde, ${ }^{117-121}$ weniger Ethanol ${ }^{122,123}$ oder tert-Butylalkohol. ${ }^{124}$

Neben den Obertönen in $\mathrm{O}-\mathrm{H} \cdots \mathrm{O}-\mathrm{H}-$ Systemen, zu denen natürlich auch das Wasserdimer ${ }^{39}$ gehört, finden sich in der Literatur viele weitere Beispiele für Obertonspektroskopie an Dimeren mit Wasserstoffbrücken in der Gasphase. So wurde etwa das gemischte Dimer von Methanol und Trimethylamin bei Raumtemperatur beobachtet. ${ }^{125}$ Die N-H Streckschwingungsobertöne des Pyrroldimers wurden in Jetexpansionen beobachtet. ${ }^{126}$

Ein weiteres wichtiges Modellsystem ist das Fluorwasserstoffdimer, dessen Obertöne ausgiebig in der Literatur untersucht wurden. ${ }^{127-132}$ Auch in gemischten Komplexen mit organischen Molekülen, ${ }^{133}$ Cyanwasserstoff, ${ }^{134}$ Argon ${ }^{129,135-141}$ und Stickstoff ${ }^{129,138,142}$ wurden HFObertöne beobachtet. Als analoge Systeme sind auch die Dimere des OH-Radikals mit Argon, ${ }^{143}$ Stickstoff ${ }^{144}$ und ferner Acetylen ${ }^{145}$ zu nennen. Obertöne des Cyanwasserstoffdimers sind ebenfalls publiziert. ${ }^{146}$ Auch das Acetylendimer selbst ${ }^{147,148}$ und das gemischte Acetylen-StickstoffDimer ${ }^{149}$ kann man als Beispiele für Systeme mit sehr schwachen Wasserstoffbrücken aufführen, deren Obertöne untersucht wurden. Daneben ist es möglich, die Obertöne der Oszillatoren in intramolekularen Wasserstoffbrücken in der Gasphase zu untersuchen. ${ }^{88,150-153}$

In kondensierter Phase wurden die Alkohole dieses Kapitels ebenfalls untersucht. Dabei treten natürlich starke Umgebungseinflüsse auf, sodass deutliche Abweichungen zu den isolierten Systemen erwartet werden können - sowohl in den reinen Substanzen als auch in Lösungen. ${ }^{154-164}$ Die höhere Substanzkonzentration vereinfacht jedoch die Messungen und ermöglicht die Messung schwacher Signale wie Kombinationsbanden benachbarter O-H Gruppen. ${ }^{165-167}$ Mit der relativ einfachen Messmethodik in kondensierter Phase ließen sich so die Eigenschaften von Wasserstoffbrücken-gebundenen Komplexen und Flüssigkeiten durch Obertonspektroskopie untersuchen. ${ }^{168,169}$ Das Einfrieren in glasartigen Matrizen bietet eine weitere Möglichkeit Alkoholobertöne zu untersuchen. ${ }^{170,171}$ In Inertgasmatrizen ${ }^{11,172}$ kann man etwas schwächere Einflüsse auf die Obertonübergänge erwarten, sie werden in Abschnitt 4.2.2 am Beispiel von Methanol genauer diskutiert.

Die Effekte der mechanischen und elektrischen Anharmonizität lassen sich in den Spektren der entsprechenden Schwingung wiederfinden und quantifizieren. Während erstere die Lage der Übergänge beeinflusst, ${ }^{10}$ ist die Intensität der Übergänge von beiden abhängig. In den in dieser Arbeit durchgeführten Jetexpansionen lassen sich Bandenzentren besonders leicht bestimmen, da die Abkühlung zu einer Vereinfachung der Rotations- und Schwingungsstruktur führt und energetisch ungünstige Konformationen der Moleküle entvölkert. Einzig die hier verwendete mäßige Auflösung von $2 \mathrm{~cm}^{-1}$ kann hinderlich sein, wie am Beispiel vom Methanolmonomer im 
Folgenden zu sehen ist. Absolute Bandenintensitäten lassen sich mit der Jet-Methode nicht bestimmen, da dazu die Konzentration der Moleküle in der Expansion bekannt sein müsste. Jedoch lässt sich auf Grund der unveränderten Expansionsbedingungen das Verhältnis der Intensitäten von Fundamentale und Oberton bestimmen. Problematisch ist dies jedoch bei sehr konzentrierten Spezies wie den Monomeren der Alkohole. Anders als die Spektren bei $2 \mathrm{~cm}^{-1}$ Auflösung mit ihrer moderaten Absorbanz vermuten lassen, liegen bei Monomeren in Wahrheit sehr scharfe Übergänge vor, deren hohe Absorbanz zu einer Sättigung der Übergangsintensitäten führt. Somit werden besonders für die intensiven Fundamentalübergänge zu geringe integrierte Intensitäten der Monomere bestimmt, während bei ihren um eine Größenordnung schwächeren Obertönen dieser Effekt weniger stark ausgeprägt ist. Die Folge sind zu klein bestimmte Intensitätsverhältnisse von Fundamentale zu Oberton in der Jetexpansion. In Gasphasenspektren lässt sich dieses Problem durch hinzufügen eines Inertgases und der damit verbundenen Stoßverbreiterung vermeiden.

Im Gegensatz zu den Monomeren liegen bei Wasserstoffbrückenclustern die Probleme der Intensitätsmessung eher in den sehr schwachen Obertonübergängen der $\mathrm{O}-\mathrm{H}$ Streckschwingung begründet, da homogene Verbreiterung der Banden die Übersättigung der Spektren verhindert. Somit sind gepulste Jetexpansionen besonders geeignet um Obertonspektroskopie an Clustern durchzuführen, da sie die entsprechenden kalten Systeme in großer Zahl präparieren, während bei den Intensitätsmessungen der Monomere eher hochaufgelöste Gasphasenmessungen vorzuziehen sind.

Um die Anharmonizitätseffekte der Dimerbildung in den Spektren unmittelbar sichtbar zu machen, werden in den folgenden Abschnitten die Oberton- und Fundamentalspektren der Alkohole direkt übereinander dargestellt - beide unter identischen Expansionsbedingungen aufgenommen. Die Wellenzahlskalen wurden für das Obertonspektrum um den Faktor 2 gestaucht und so verschoben, dass die Monomerübergänge von Oberton- und Fundamentalspektrum in den Abbildungen an der gleichen Position liegen, sodass sich Änderungen der diagonalen Anharmonizitäten der Dimerschwingungen relativ zum Monomer direkt als Differenzen zwischen den entsprechenden Dimerübergängen beider Spektren visuell manifestieren. Die Absorbanzskalen wurden so abgestimmt, dass die Monomerbanden in Oberton- und Fundamentalspektrum die gleiche Fläche einnehmen. Somit werden die Änderungen der Dimerintensitäten relativ zum Monomer ebenfalls quantitativ sichtbar gemacht.

\subsubsection{Messbedingungen}

Die Spektren der Überschallexpansionen von Alkoholen wurden bei $60 \mathrm{kHz}$ Spiegelgeschwindigkeit und $2 \mathrm{~cm}^{-1}$ Auflösung gemessen. Die Konzentrationen und Stagnationsdrücke wurden für die Dimerspektren so gewählt, dass sich eine maximale Dimerintensität in den Spektren ergibt. Dieses Optimum liegt beim Filet-Jet je nach Substanz bei einem Druck von 0.8 bis 0.9 bar und einer aus den Gleichgewichtsdampfdrücken (1.6 bar Heliumdruck im Sättiger) abgeschätzten Konzentration von 0.3 bis $0.6 \%$ im Reservoir (Vgl. Tabelle 4.3). Für die Optiken wurde für Fenster, Linsen und Strahlteiler durchgehend $\mathrm{CaF}_{2}$ verwendet und als Lichtquelle die Wolframlampe des Spektrometers. Spektren im Fundamental- und Obertonbereich der O-H Streckschwingung ließen sich gleichzeitig aufnehmen, wofür das $2 \mathrm{~mm}$ InSb-Detektorelement eines InSb/MCT- 


\section{4. $O-H$ Streckschwingungen}

Tabelle 4.3.: Experimentelle Bedingungen der Alkoholspektren bei maximaler Dimerintensität: Stagnationsdruck $p_{\text {st }}$, Sättigertemperatur $T_{\mathrm{S}}$ und Verdünnung auf welche die Helium/Substanz-Mischung des Sättigers mit Hilfe der Zumischung von Helium (über eine weitere Zuleitung zum Reservoir) reduziert wurde. Aus den integrierten Bandenintensitäten $\int_{\nu_{02}} \ln \left(\frac{I_{0}}{I_{\mathrm{M}+\mathrm{D}_{\mathrm{a}}}}\right) \mathrm{d} \tilde{\nu}$ des InSb- und InGaAs-Detektors über den Obertonbereich der Monomere und Dimerakzeptoren ergibt sich der Faktor $F\left(\frac{\mathrm{InSb}}{\operatorname{InGaAs}}\right)$.

\begin{tabular}{|c|c|c|c|c|c|c|}
\hline & $\begin{array}{l}p_{\mathrm{st}} \\
/ \text { bar }\end{array}$ & $\begin{array}{r}T_{\mathrm{S}} \\
/{ }^{\circ} \mathrm{C}\end{array}$ & $\begin{array}{c}\text { Ver- } \\
\text { dünnung }\end{array}$ & $\begin{array}{c}\int_{\nu_{02}} \ln \left(\frac{I_{0}}{I_{\mathrm{M}+\mathrm{D}_{\mathrm{a}}}}\right) \mathrm{d} \tilde{\nu} \\
/ 10^{-3} \mathrm{~cm}^{-1}(\mathrm{InSb})\end{array}$ & $\begin{array}{c}\int_{\nu_{02}} \ln \left(\frac{I_{0}}{I_{\mathrm{M}+\mathrm{D}_{\mathrm{a}}}}\right) \mathrm{d} \tilde{\nu} \\
/ 10^{-3} \mathrm{~cm}^{-1}(\operatorname{InGaAs})\end{array}$ & $F\left(\frac{\operatorname{InSb}}{\operatorname{InGaAs}}\right)$ \\
\hline $\mathrm{MeOH}$ & 0.8 & -13 & $50 \%$ & $3.94(73)$ & $3.10(9)$ & $0.79(15)$ \\
\hline EtOH & 0.9 & -5 & $50 \%$ & $1.88(22)$ & $1.24((4)$ & $0.66(8)$ \\
\hline $\mathrm{EtOH}+1.5 \% \mathrm{Ar}$ & 0.9 & -5 & $50 \%$ & $1.11(24)$ & $1.10(2)$ & $0.99(21)$ \\
\hline$t \mathrm{BuOH}$ & 0.8 & 26 & $7 \%$ & $1.16(40)$ & $0.80(11)$ & $0.69(25)$ \\
\hline HFIP & 0.8 & 0 & $20 \%$ & $4.4(1.1)$ & $3.35(30)$ & $0.77(21)$ \\
\hline Mittelwert & & & & & & $0.78(13)$ \\
\hline
\end{tabular}

Sandwichdetektors (77 K, „Sandwich_neu“) ohne weiteren Filter verwendet wurde. Das Signalzu-Rauschen Verhältnis reicht aber in diesem Fall nicht aus um Dimerbanden im Obertonbereich zu beobachten. Zu diesem Zweck wurde ein $3 \mathrm{~mm}$ InGaAs Detektor (Raumtemperatur) in Kombination mit Filter $16\left(6400-7400 \mathrm{~cm}^{-1}\right)$ verwendet. Dabei ergaben sich beim Vergleich der Spektren von InSb- und InGaAs-Detektor auf Grund der verschiedenen Elementflächen und verschiedener verwendeter Aperturen (InSb: $1.5 \mathrm{~mm}$, InGaAs: $4 \mathrm{~mm}$ ) unterschiedlich starke Intensitäten für die Obertonbanden der Monomere. Dieser Effekt der Detektoren, der aus der Abbildung unterschiedlich großer Teile der Expansion herrührt, muss für die Auswertung der Dimerintensitäten berücksichtigt werden, da für die Verhältnisse von Fundamentale zu Oberton die Spektren beider Detektortypen verglichen werden. In Tabelle 4.3 sind neben anderen experimentellen Angaben die Intensitäten der Monomerobertöne für beide Detektoren dargestellt, wobei der Wert für den InSb-Detektor auf Grund des höheren Rauschens stärker fehlerbehaftet ist. Dafür wurde der gesamte Monomerbereich (inklusive der Dimerakzeptoren) der jeweiligen Substanzen integriert (bei Hexafluorisopropanol wurde das gauche-Monomer nicht mitintegriert). Daraus ergibt sich jeweils der Faktor, um den sich die Intensität des InGaAs-Detektors für die O-H Obertonbande von der Intensität des InSb-Detektors unterscheidet. Der Mittelwert $\bar{F}\left(\frac{\mathrm{InSb}}{\operatorname{InGaAs}}\right)=0.78(13)$ wird dann als allgemeiner Korrekturfaktor für die Auswertung der Dimerintensitätsverhältnisse aller Alkohole verwendet, unter der Annahme dass dieser Faktor nur wenig substanzspezifisch und der Einfluss der leicht unterschiedlichen Expansionsbedingungen gering ist. Ferner muss angenommen werden, dass der Faktor von den Monomer- auf die Dimersignale übertragbar ist. Die Abweichungen der einzelnen Faktoren von diesem Mittelwert wären damit vor allem aus dem schlechten Signal-zu-Rauschen Verhältnis der InSb-Messungen zu erklären. Der publizierte Mittelwert ${ }^{11}$ von 0.78(15) wurde mit einem etwas größeren Fehlerbereich versehen, da dort Hexafluorisopropanol nicht in die Analyse mit einbezogen wurde. 

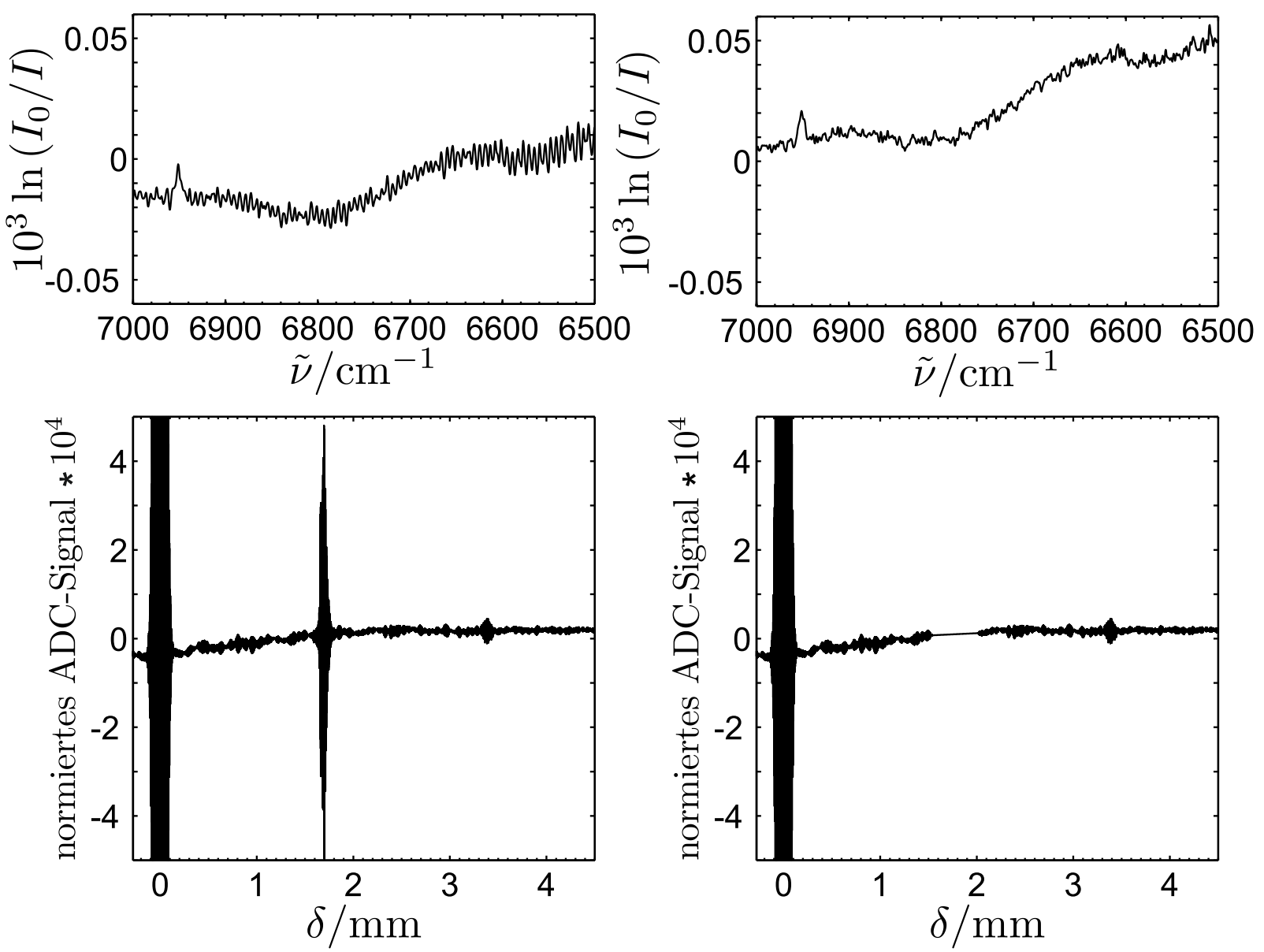

Abbildung 4.9.: Probeninterferogramme von Methanolexpansionen (800 gemittelte Scans) und entsprechende Spektren. Links: Unbearbeitetes Interferogramm (unten) mit Hauptmaximum bei einer Spiegelauslenkung von $0 \mathrm{~mm}$ und einem Nebenmaximum bei $1.7 \mathrm{~mm}$. Das Spektrum (oben) zeigt deutlich ein Interferenzmuster im Bereich des Dimerdonorobertons bei $6951 \mathrm{~cm}^{-1}$. Rechts: Nach ersetzen des Nebenmaximums durch eine Linie im Interferogramm (unten), ist die Interferenz im Spektrum (oben) beseitigt. Es ergibt sich im Vergleich zum unmanipulierten Spektrum ein positiver Off-set der Absorbanz.

Eine Komplikation bei der Messung der Spektren ergab sich durch das Auftreten einer Interferenz im Bereich des Dimerdonorobertons, die das schwache Substanzsignal überlagern konnte. Diese Interferenz manifestierte sich sowohl in den Interferogrammen des Hintergrunds als auch der Probe in Form eines ausgeprägten Nebenmaximums, zusätzlich zum Hauptmaximum. Die Störung konnte behoben werden, indem der betroffene Teil des Interferogramms durch eine gerade Linie ersetzt wurde. In Abbildung 4.9 ist die Bearbeitung der Interferogramme am Beispiel von Methanolspektren dargestellt. Eine Erzeugung von Artefakten im Spektrum durch die Manipulation wurde weitestgehend vermieden, indem für Anfangs- und Endpunkte der Linien Stellen 


\section{4. $O-H$ Streckschwingungen}

des Interferogramms gewählt wurden, bei denen das ADC-Signal möglichst schwach oszilliert. Obwohl die Interferogramme von Proben- und Hintergrundscans gleichartig bearbeitet wurden, ergibt sich in den resultierenden Spektren ein geringer Off-set gegenüber den Originalspektren.

\subsubsection{Methanol}

Die innere Rotation der Hydroxylgruppe in Methanol wird über Tunneln durch das Torsionspotential realisiert und führt zu einer Aufspaltung der Schwingungszustände, was in Kapitel 5.2.1 näher beschrieben wird. Außerdem hat Methanol eine vergleichsweise große Rotationskonstante um die $\mathrm{C}-\mathrm{O}$ Bindung und es liegt eine starke Kopplung zwischen den Rotations- und $\mathrm{O}-\mathrm{H}$ Streckschwingungsniveaus vor. Diese Umstände führen zu breiten Banden der $\mathrm{O}-\mathrm{H}$ Streckschwingung im Gasphasenspektrum, bestehend aus zahlreichen Übergängen, die von verschiedenen Torsions- und Rotationsniveaus ausgehen. Dadurch ist die Zuordnung der Bandenzentren im Fundamental- und Obertonbereich nicht trivial und nur unter Rotationsauflösung möglich. Die Kühlung in einer Überschallexpansion reicht nicht aus um die IR-Spektren auf hinreichend wenige Übergänge einzuschränken.

Tabelle 4.4.: Experimentelle Bandenzentren der Fundamentale $\tilde{\nu}_{\mathrm{OH}}$ und ersten Obertons $2 \tilde{\nu}_{\mathrm{OH}}$, sowie ermittelte diagonale $\mathrm{O}-\mathrm{H}$ Anharmonizität des Methanomonomers $x_{\mathrm{OH}, \mathrm{OH}}^{\mathrm{M}}$

\begin{tabular}{clllc}
\hline & $\tilde{\nu}_{\mathrm{OH}} / \mathrm{cm}^{-1}$ & $2 \tilde{\nu}_{\mathrm{OH}} / \mathrm{cm}^{-1}$ & $x_{\mathrm{OH}, \mathrm{OH}}^{\mathrm{M}} / \mathrm{cm}^{-1}$ & \\
\hline I & & $-86(1)^{117}$ & Birge-Sponer Auftragung \\
II & & & $-86.3(5)^{118}$ & Birge-Sponer Auftragung \\
III & & & $-86.2(7)^{121}$ & Birge-Sponer Auftragung \\
IV & & & $-85.2(2)^{173}$ & anharmonische Modellierung \\
V & $3685.32(1)^{174}$ & $7198.67(1)^{120}$ & $-85.99(1)$ & A-A Übergänge \\
VI & $3678.0^{174}(8)$ & $7186.2(4)^{120}$ & $-84.9(6)$ & aus den Potentialminima der Torsion \\
VII & $3684.0(2)$ & $7196.27(1)$ & $-85.8(2)$ & ungewichtete Mittelung über A und E \\
VIII & $3683.6(2)$ & $7195.47(1)$ & $-85.9(2)$ & Mittelung über A und zweimal E \\
\hline & $\mathbf{3 6 8 4 ( 1 )}$ & $\mathbf{7 1 9 6 ( 2 )}$ & $\mathbf{- 8 5 . 8 ( 6 )}$ & in dieser Arbeit verwendete Werte \\
\hline
\end{tabular}

Deshalb wurde bei der Bestimmung der diagonalen Anharmonizität $x_{\mathrm{OH}, \mathrm{OH}}^{\mathrm{M}}$ der $\mathrm{O}-\mathrm{H}$ Streckschwingung von Methanol in der frühen Literatur die mangelnde Auflösung durch Mittelung über mehrere Übergänge ausgeglichen. Tabelle 4.4 fasst die Ergebnisse für $x_{\mathrm{OH}, \mathrm{OH}}^{\mathrm{M}}$ aus verschiedenen Ansätzen zusammen. So wurden aus Birge-Sponer Auftragungen über den zweiten bis fünften Oberton ${ }^{117}$ (I), sowie Fundamentale bis fünften Oberton ${ }^{118}$ (II), bzw. bis zum siebten Oberton $^{121}$ (III) gut übereinstimmende Ergebnisse von $x_{\mathrm{OH}, \mathrm{OH}}^{\mathrm{M}} \approx-86 \mathrm{~cm}^{-1}$ gefunden. Weiter geht eine anharmonische Modellierung von Methanol (IV) unter Berücksichtigung experimenteller Daten zu fast allen Schwingungen, unter anderem bis hin zum sechsten Oberton der $\mathrm{O}-\mathrm{H}$ Streckschwingung, ergänzt durch quantenchemische Vorhersagen. ${ }^{173}$ Dabei ergibt sich eine um $1 \mathrm{~cm}^{-1}$ geringere Anharmonizitätskonstante für die $\mathrm{O}-\mathrm{H}$ Streckschwingung. Diese Ansätze bieten eine Beschreibung der niedrigen Quantenzustände der $\mathrm{O}-\mathrm{H}$ Streckschwingung. Bei höherer Anregung kann der Einfluss von höheren Anharmonizitätskonstanten signifikanter werden, die 
möglicherweise schon bei den oben genannten Studien zu einem geringen Teil in $x_{\mathrm{OH}, \mathrm{OH}}^{\mathrm{M}}$ hinein gemittelt wurden.

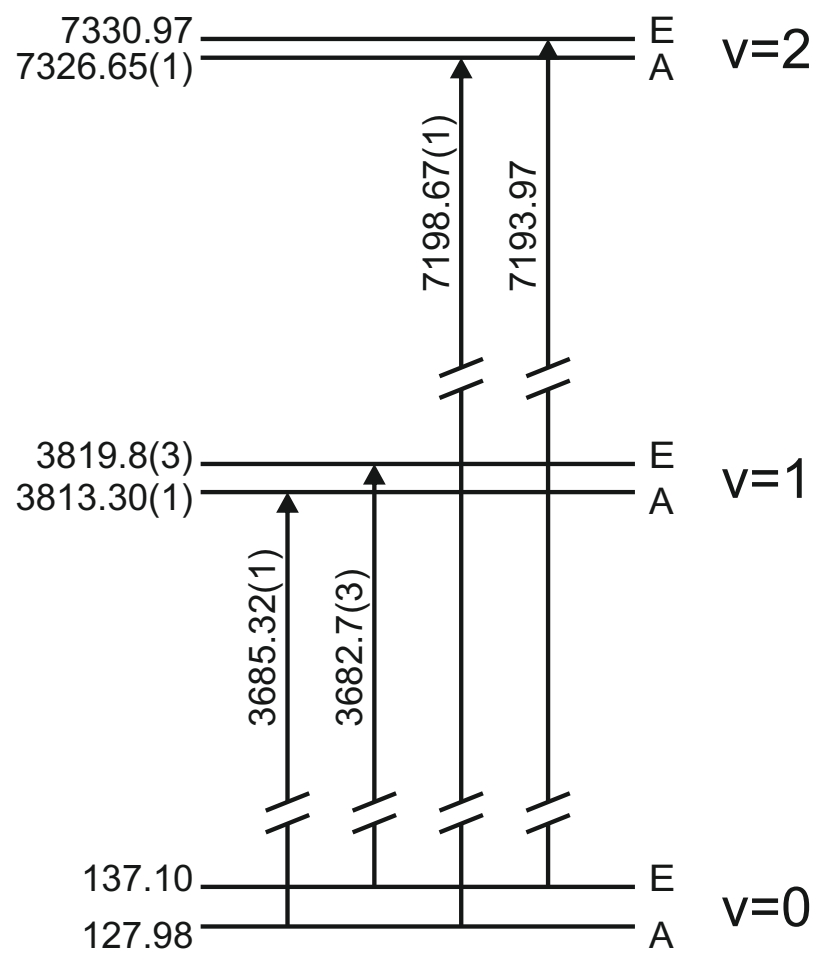

Abbildung 4.10.: Energieniveaus (in $\mathrm{cm}^{-1}$ ) der OH-Streckschwingung von Methanol in $\mathrm{v}=$ $0,{ }^{175} 1^{174}$ und $2^{120}$ im Rotationsgrundzustand ( $\mathrm{K}=0$ und $\mathrm{J}=0$, entsprechend der Nomenklatur in Referenz 174). Die Energien sind inklusive der Nullpunktsenergie der $\mathrm{O}-\mathrm{H}$ Torsion im Schwingungsgrundzustand angegeben. Es ergeben sich die Wellenzahlen der entsprechenden $\mathrm{A}-\mathrm{A}$ und $\mathrm{E}-\mathrm{E}$ Übergänge.

Alternativ zur Mittelung über mehrere Übergänge kann bei genauer Kenntnis der Wellenzahlen von Fundamentale und erstem Oberton aus diesen $x_{\mathrm{OH}, \mathrm{OH}}^{\mathrm{M}}$ ermittelt werden, was zu einer genaueren Beschreibung der Schwingungsniveaus nahe am Potentialminimum führen kann und auch dem Vorgehen bei der anschließenden Untersuchung des Methanoldimers entspricht. Diese Beschränkung auf wenige Zustände ist jedoch anfälliger gegenüber Verfälschungen durch auftretende Resonanzen zwischen den Schwingungs-Rotationszuständen und dunklen Zuständen. ${ }^{174}$ In Abbildung 4.10 sind die beteiligten Niveaus der $\mathrm{O}-\mathrm{H}$ Streckschwingung von Methanolmonomer gezeigt, die aus rotationsaufgelösten Messungen von Methanol erhalten wurden. ${ }^{120,174,175}$ Sie werden durch die Torsion der O-H Gruppe in einen A und zwei E Zustände (entartet wenn $\mathrm{K}=0)^{174}$ aufgespalten. Die sich daraus ergebenden Fundamentalübergänge unter Erhalt der Tunnelsymmetrie sind im Jet-gekühlten Ramanspektrum als intensive Peaks sichtbar. ${ }^{36}$ In Tabelle 4.4 sind verschiedene Möglichkeiten zur Bestimmung von $x_{\mathrm{OH}, \mathrm{OH}}^{\mathrm{M}}$ aus diesen Daten angegeben. Eine reine Betrachtung der A-A Übergänge ${ }^{121,174}(\mathrm{~V})$ führt $\mathrm{zu} x_{\mathrm{OH}, \mathrm{OH}}^{\mathrm{M}}=-85.99 \mathrm{~cm}^{-1}$. Alternativ lässt sich der Einfluss der Torsion auch ganz herausrechnen, wenn nur die Energie- 


\section{4. $O-H$ Streckschwingungen}

unterschiede zwischen den Minima der Torsionspotentiale betrachtet werden ${ }^{120,174}$ (VI), die sich aus Fits an die Schwingungs-Rotationsniveaus von Methanol ergeben. Daraus ergibt sich die geringste Anharmonizität aller betrachteten Methoden. Um den Effekt der Tunnelaufspaltung aufzuheben kann auch über die E und A Zustände in Abbildung 4.10 gemittelt werden, entweder ungewichtet (VII) (Vgl. Referenz 121) oder unter doppelter Gewichtung der entarteten Energie der E-Zustände (VIII). Daraus ergeben sich Werte für $x_{\mathrm{OH}, \mathrm{OH}}^{\mathrm{M}}$ knapp unter $-86 \mathrm{~cm}^{-1}$.

Da aus den in dieser Arbeit durchgeführten Messungen von Methanol Monomer kein Wert $x_{\mathrm{OH}, \mathrm{OH}}^{\mathrm{M}}$ ermittelt werden kann, wird im folgenden für $x_{\mathrm{OH}, \mathrm{OH}}^{\mathrm{M}}=-85.8(6)$ angenommen. Dieser Wert berücksichtigt alle Literaturdaten $\mathrm{zu} x_{\mathrm{OH}, \mathrm{OH}}^{\mathrm{M}}$ in Tabelle 4.4, ausgenommen dem torsionsentkoppelten Ansatz (VI). Die Birge-Sponer Auftragungen (I-III) liegen innerhalb der höheren, das anharmonische Modell (IV) in den unteren Fehlergrenzen und die Betrachtung der gemittelten Fundamentalen und ersten Obertöne (VII und VIII) stimmt gut mit dem verwendeten Wert überein. Für das effektive Bandenzentrum der Fundamentale kann man auf Grundlage von V, VII und VIII vorsichtig 3684(1) $\mathrm{cm}^{-1}$ festlegen und entsprechend den Oberton bei 7196(2) $\mathrm{cm}^{-1}$ verorten. Diese Wahl weicht etwas von dem Resultat der in Referenz 11 publizierten Analyse der Literaturwerte $a b$.

Wie eingangs von Abschnitt 4.2 erläutert, sind die Intensitätsmessungen von Monomeren am Filet-Jet fehlerbehaftet, weshalb hier für Monomerdaten auf die Literatur zurückgegriffen werden muss. Für Methanol in der Gasphase wurde für das Fundamentale-zu-Oberton Verhältnis ein Wert von 12.1(9) ermittelt. ${ }^{116}$ Im Vergleich dazu ergibt sich durch Integration der Monomerbanden aus den Jet-Messungen ein Verhältnis von 6(1). Der Fehler der sich durch mitintegrieren der Akzeptorbande ergibt, kompensiert den stärkeren Effekt der Übersättigung wahrscheinlich ein wenig.

In Abbildung 4.11 sind die Spektren der $\mathrm{O}-\mathrm{H}$ Streckschwingung von Jetexpansionen von $0.5 \%$ Methanol in 0.8 bar Helium gezeigt, ${ }^{11}$ die auch Signale des Dimers enthalten. Im Fundamentalspektrum sind die Banden des Monomers (M), Dimerdonors $\left(\mathrm{D}_{\mathrm{d}}\right)$ und Trimers $(\mathrm{T})$ klar erkennbar (Strukturen in Abb. 2.1), die bereits zugeordnet und im Falle der Letzteren bezüglich ihrer Struktur analysiert wurden. ${ }^{36}$ Die Fundamentale der Dimerdonorbande liegt bei $3574.5(3) \mathrm{cm}^{-1}$ woraus sich eine Rotverschiebung zum Monomer von $110(1) \mathrm{cm}^{-1}$ ergibt. Die Bande des Dimerakzeptors liegt im Bereich der breiten Monomerbande und wurde bisher bei $3684.1 \mathrm{~cm}^{-1}$ im größenselektiven Helium-Streuexperiment ${ }^{44}$ und bei $3683.1 \mathrm{~cm}^{-1}$ mittels unselektiver Cavity-Ring-Down-Spektroskopie ${ }^{50}$ beobachtet. Dagegen wurde unter Anwendung von größenselektiver IR-VUV-Ionisation der Dimerakzeptor bei $3675 \mathrm{~cm}^{-1}$ gefunden. ${ }^{51}$ In den in dieser Arbeit durchgeführten Experimenten konnten keine Hinweise auf die $\mathrm{O}-\mathrm{H}$ Streckschwingung des Dimerakzeptors gefunden werden.

Im Obertonspektrum in Abbildung 4.11 ist vor allem die Monomerbande gut erkennbar, die nur um eine Größenordnung weniger intensiv als die Fundamentale erscheint. Die Bande des Dimerdonors ist bei 6950.6(6) $\mathrm{cm}^{-1}$ zu finden - erheblich abgeschwächt gegenüber dem Fundamentalübergang, der um einen Faktor 320(80) intensiver ist. Aus den zugehörigen Bandenpositionen ergibt sich eine Anharmonizitätskonstante des Dimerdonors $x_{\mathrm{OH}, \mathrm{OH}}^{\mathrm{D}_{\mathrm{d}}}$ von $-99.2(4) \mathrm{cm}^{-1}$ für die O-H Streckschwingung. Dies entspricht einer Steigerung von 15.6(9)\% gegenüber dem Monomer. Somit stellt sich das Fundamentale-zu-Oberton Verhältnis mit einer Erhöhung um 2500(700)\% als deutlich ausgeprägter und empfindlicher auf die Ausbildung der Wasserstoff- 

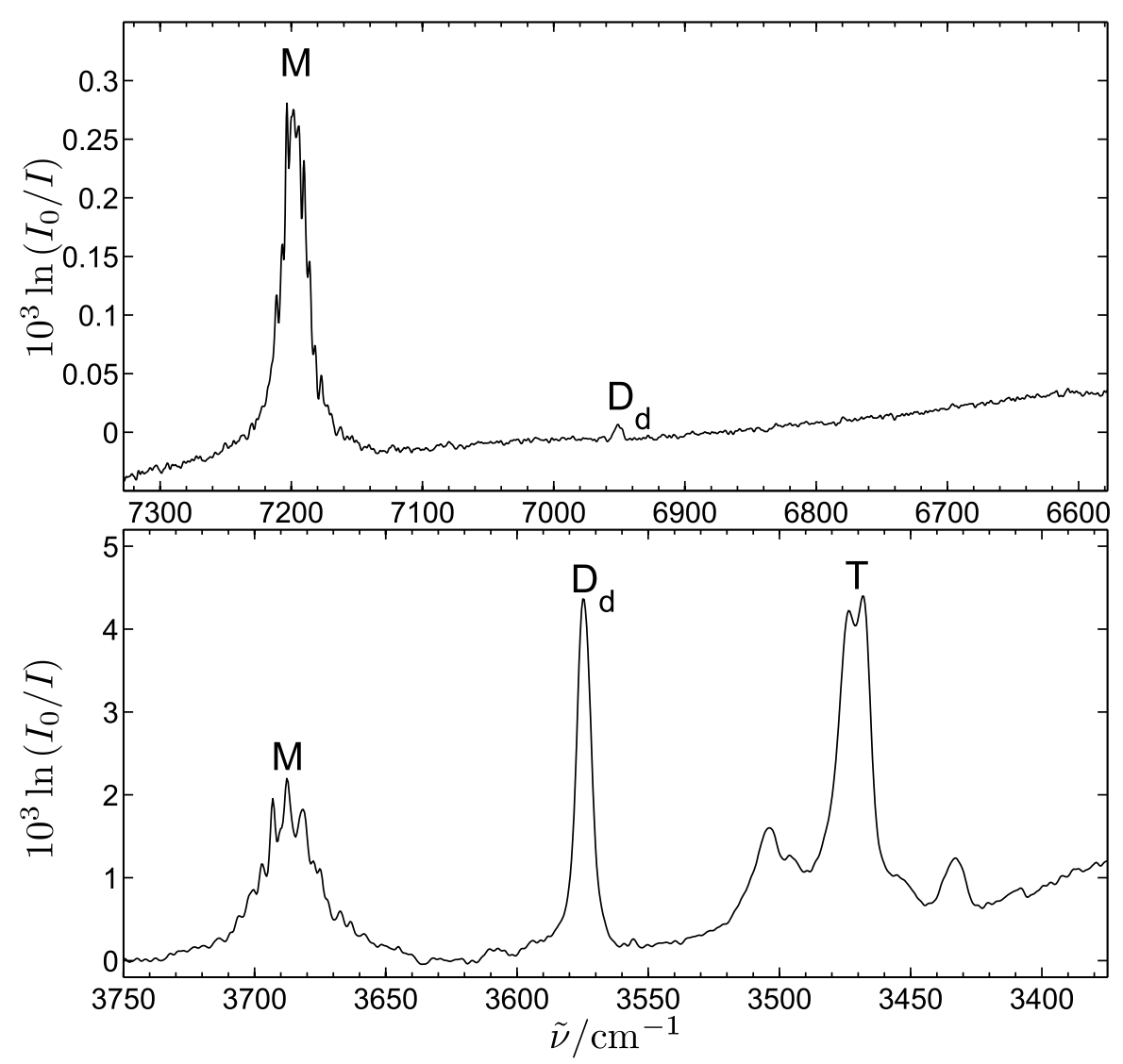

Abbildung 4.11.: Oberton- (oben, 1450 Pulse) und Fundamentalspektrum (unten, 200 Pulse) der Jetexpansionen von $0.5 \%$ Methanol in 0.8 bar Helium im Bereich der $\mathrm{O}-\mathrm{H}$ Streckschwingung. Im Fundamentalspektrum sind die Banden von Monomer $(\mathrm{M})$, Dimerdonor $\left(\mathrm{D}_{\mathrm{d}}\right)$ und Trimer $(\mathrm{T})$ klar zu erkennen, die Bande des Dimerakzeptors wird von der Monomerbande überdeckt. Im Obertonspektrum ist die Dimerdonorbande sehr schwach und Trimerbanden gar nicht zu erkennen.

brücke im Dimer heraus. Da für die Wasserstoffbrücken im Trimer auf Grund kooperativer Wechselwirkungen dieser Intensitätseffekt noch ausgeprägter erwartet werden kann, ist es nicht verwunderlich dass keine Trimerbanden im Obertonspektrum beobachtet werden können. Die Signale des Trimers müssen mindestens um einen Faktor von 1200 schwächer sein als die IRintensivste Trimerfundamentale. Für die Trimere ließen sich im Obertonspektrum sowohl Obertöne als auch Kombinationsbanden der $\mathrm{O}-\mathrm{H}$ Streckschwingungen erwarten. Auch für das Dimer sollte ein Kombinationsübergang vorliegen, bestehend aus den $\mathrm{O}-\mathrm{H}$ Streckschwingungen des Donors und Akzeptors. Die Addition der Wellenzahlen ihrer Fundamentalen lässt ihren gemeinsamen Übergang in der höherenergetischen Flanke des Monomerobertons vermuten, was die Detektion dieser vermutlich ohnehin äußerst schwachen Kombinationsbande erschwert. In Tabelle 4.5 sind die spektroskopischen Daten aus Jetexpansionen für das Methanolmonomer und -dimer zusammengestellt. 


\section{4. $O-H$ Streckschwingungen}

Tabelle 4.5.: Spektroskopische Daten aus Methanolexpansionen: Bandenzentren der $\mathrm{O}-\mathrm{H}$ Streckschwingung des Dimerdonors $\left(\mathrm{D}_{\mathrm{d}}\right)$ in $\mathrm{cm}^{-1}$ und integrierte Absorbanzen $\int 10^{3} \ln \left(\frac{I_{0}}{I}\right) \mathrm{d} \tilde{\nu}$ in $\mathrm{cm}^{-1}$ (kursiv gedruckt) der Banden von Monomer (M) (inklusive Dimerakzeptorabsorbanz) und Dimerdonor. Bei der Berechnung der Fundamentale-zu-Oberton Verhältnisse müssen die integrierten Absorbanzen der Fundamentalen mit dem Korrekturfaktor von 0.78(13) multipliziert werden.

\begin{tabular}{lll}
\hline & $\mathrm{M}$ & $\mathrm{D}_{\mathrm{d}}$ \\
\hline$\nu_{\mathrm{OH}}$ & & $3574.5(3)$ \\
& $25(1)$ & $14.5(8)$ \\
$2 \nu_{\mathrm{OH}}$ & & $6950.6(6)$ \\
& $3.5(1)$ & $0.035(6)$ \\
\hline \hline
\end{tabular}

Tabelle 4.6.: Vergleich der Methanoldaten zur O-H Streckschwingung in verschiedenen Kryomatrizen mit Gasphasen- und Jetexpansionsmessungen: Monomerfundamentale $\tilde{\nu}_{\mathrm{OH}}^{\mathrm{M}}$, Verschiebung der Dimerdonorfundamentale zum Monomer $\Delta \tilde{\nu}_{\mathrm{OH}}^{\mathrm{D}_{\mathrm{d}}-\mathrm{M}}$, diagonale Anharmonizität des Monomers $x_{\mathrm{OH}, \mathrm{OH}}^{\mathrm{M}}$ und des Dimerdonors $x_{\mathrm{OH}, \mathrm{OH}}^{\mathrm{D}_{\mathrm{d}}}$ (alle in $\mathrm{cm}^{-1}$ ), sowie das Fundamentale-zu-Oberton Intensitätsverhältnis des Monomers $\frac{S_{01}^{\mathrm{M}}}{S_{02}^{\mathrm{M}}}=\frac{\int \ln \left(\frac{I_{0}}{I_{\mathrm{M}}}\right) \mathrm{d} \tilde{\nu}}{\int_{\nu_{02}} \ln \left(\frac{I_{0}}{I_{\mathrm{M}}}\right) \mathrm{d} \tilde{\nu}}$ und des Dimerdonors $\frac{S_{01}^{\mathrm{D}_{\mathrm{d}}}}{S_{02}^{\mathrm{D}_{\mathrm{d}}}}$. Die Daten des Dimerdonors sind jeweils nur für die intensivste Bande in den Spektren angegeben.

\begin{tabular}{|c|c|c|c|c|c|c|}
\hline & $\tilde{\nu}_{\mathrm{OH}}^{\mathrm{M}}$ & $\Delta \tilde{\nu}_{\mathrm{OH}}^{\mathrm{D}_{\mathrm{d}}-\mathrm{M}}$ & $x_{\mathrm{OH}, \mathrm{OH}}^{\mathrm{M}}$ & $x_{\mathrm{OH}, \mathrm{OH}}^{\mathrm{D}_{\mathrm{d}}}$ & $\frac{S_{01}^{\mathrm{M}}}{S_{02}^{\mathrm{M}}}$ & $\frac{S_{01}^{\mathrm{D}_{\mathrm{d}}}}{S_{02}^{\mathrm{D}_{\mathrm{d}}}}$ \\
\hline Gasphase/Jet & $3684(1)$ & $-110(1)$ & $-85.8(6)$ & $-99.2(4)$ & $12.1(9)^{116}$ & $320(80)$ \\
\hline Ne-Matrix ${ }^{176,177}$ & $3688.0,3690.2$ & & -86.4 & & & \\
\hline Ne-Matrix ${ }^{11}$ & 3689.3 & -129.3 & -86 & -98 & 11(3) & $300(150)$ \\
\hline Ar-Matrix ${ }^{172}$ & 3666.3 & -139.6 & -85.9 & -102.0 & 12.5 & 400 \\
\hline$p$ - $\mathrm{H}_{2}$-Matrix ${ }^{11}$ & 3671.2 & -132.7 & -86 & -101.6 & $9(3)$ & $300(100)$ \\
\hline $\mathrm{N}_{2}$-Matrix ${ }^{172,177}$ & 3663.9 & -159.2 & -84.5 & -104.8 & 20 & 400 \\
\hline
\end{tabular}

Alternativ zur Erzeugung der Dimere in Jetexpansionen können diese auch in Kryomatrizen präpariert werden. Damit lassen sich die Systeme bei höherer Konzentration und für längere Zeiten messen. Dafür müssen aber die Einflüsse der Matrixumgebung in Kauf genommen werden, die zu einer Verschiebung der Wellenzahlen und Aufspaltung der Signale durch Moleküle in unterschiedlichen Matrixtaschen führen können. In Tabelle 4.6 sind die O-H Streckschwingungsdaten aus Messungen von Methanol in verschiedenen Matrizen zusammengetragen. Dabei lässt sich erkennen, dass in Neonmatrizen ${ }^{11,177}$ die Anharmonizitätskonstanten und Intensitätseffekte des Monomers und des Dimerdonors sehr nah an den Gasphasendaten liegen. Auch die Position der Monomerfundamentale liegt nah am Gasphasenwert. Einzig die Verschiebung des Dimerdonors zum Monomer ist deutlich größer als in den isolierten Systemen. Außerdem ist die Bande des Dimerdonors durch den Einfluss unterschiedlicher Matrixumgebungen in mehrere Peaks aufgespalten. Etwas stärker weichen die Daten aus Argon- ${ }^{172}$ und para-Wasserstoffmatrizen ${ }^{11}$ ab. 
Hier liegt der Monomerübergang bei etwas geringerer Energie, während der Effekt der Wasserstoffbrücke auf Anharmonizität und Fundamentale-zu-Oberton Intensitätsverhältnis des Dimerdonors etwas stärker ausfällt als in der Gasphase. Die Verschiebung des Dimerdonors zum Monomer fällt außerdem größer aus als in Neon. Jedoch ist die Dimerdonorbande in paraWasserstoff deutlich weniger aufgespalten als in Neon. Die Effekte der Matrixumgebung sorgen in Stickstoff ${ }^{172,177}$ für die größten Unterschiede zu den Gasphasenwerten. Besonders die geringe Wellenzahl der Monomerfundamentale und ihr größeres Fundamentale-zu-Oberton Verhältnis spricht für die Ausbildung einer schwachen Wasserstoffbrücke zwischen Methanol und Stickstoff. Jedoch liegt eine geringere diagonale Anharmonizität des Monomers als in der Gasphase vor. Durch kooperative Wechselwirkung mit der Matrix werden auch die Effekte der Wasserstoffbrücke auf die O-H Streckschwingung des Dimerdonors besonders ausgeprägt deutlich: es liegen in Stickstoff die größte Verschiebung zum Monomer, die größte diagonale Anharmonizität und das größte Fundamentale-zu-Oberton Intensitätsverhältnis vor. Die schmaleren Banden in Kryomatrizen erlauben teilweise auch die Detektion der Dimerakzeptorbande, für die in Stickstoff eine geringe Rotverschiebung zum Monomer und in Argon eine Blauverschiebung beobachtet wurde, die anderen hier diskutierten spektroskopischen Konstanten der O-H Streckschwingung des Akzeptors werden von der Wasserstoffbrïcke aber nur gering beeinflusst. ${ }^{172}$

Insgesamt kann also am Beispiel von Methanol gezeigt werden, dass Neonmatrizen die beste Näherung an das isolierte Molekül beziehungsweise Dimer bieten, wenn es um die Untersuchung der $\mathrm{O}-\mathrm{H}$ Streckschwingung geht. Während Argon- und para-Wasserstoffumgebungen größere Abweichungen zeigen und Stickstoff erwartungsgemäß den größten Matrixeffekt zeigt.

Um den Akzeptor des Dimers im Fundamentalbereich auch am Filet-Jet zu beobachten, könnte versucht werden die Auflösung der Spektren zu verbessern oder eine Untersuchung warmer Expansionen analog zu Referenz 50 unternommen werden. Dazu könnte es schon ausreichen, das Puffervolumen zu verringern. Alternativ könnte auch die Synchronisation verändert werden, um die wärmere Nachexpansion nach Schließen der Düsenventile des Filet-Jet zu messen. Wegen der schmaleren Monomerbanden bietet sich für die Fundamentale des Dimerakzeptors auch eine genauere Analyse mittels Ramanspektroskopie von Methanol in Jetexpansionen an.

\subsubsection{Ethanol}

Das Torsionspotential der O-H Gruppe von Ethanol weist im isolierten Molekül drei Minima auf. Das globale Minimum entspricht der trans-Konformation in $180^{\circ}$-Stellung zur C-C Bindung, während die gauche Konformere bei einem Diederwinkel von etwa $60^{\circ}$ und $300^{\circ}$ die lokalen Minima mit identischer Energie darstellen (Vgl. Abb. 4.12). Innerhalb der Potentialkurve der $\mathrm{O}-\mathrm{H}$ Torsion führt das zu einer Aufspaltung der gauche-Schwingungszustände durch Tunneldynamik zwischen den beiden chiralen Minima, während das trans-Konformer davon unbetroffen bleibt.

In IR-Spektren der warmen Gasphase überlappen die Banden der O-H Streckschwingung von trans- und gauche-Ethanol. In Jetexpansionen werden die Banden deutlich schmaler, was bei der Zuordnung der Bandenzentren hilft. In Abbildung 4.13 sind die Monomerspektren von Ethanol im Fundamental- und Obertonbereich dargestellt. Das Gasphasenspektrum wurde am Bruker Vertex 70 der Chili-Jetapparatur ${ }^{67}$ mit bei einer Auflösung von $0.35 \mathrm{~cm}^{-1}$ aufgenom- 

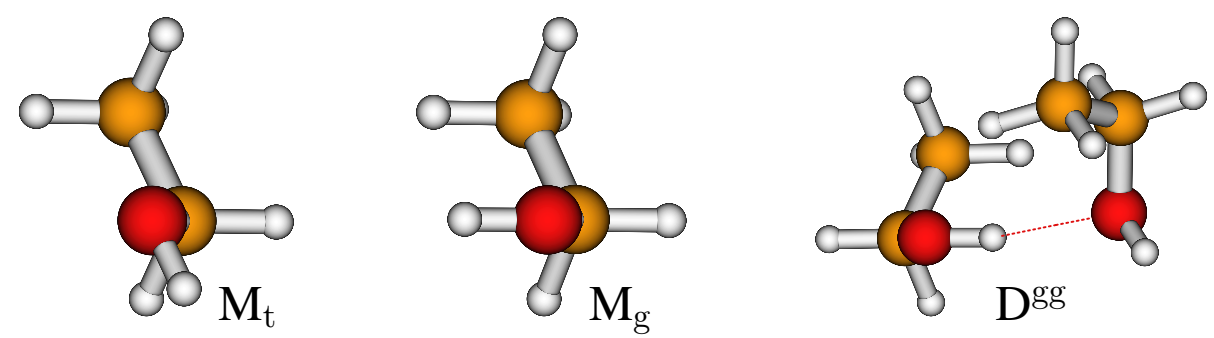

Abbildung 4.12.: Optimierte Strukturen (B3LYP-D3/6-311+G(2d,p)) von Ethanol: das transMonomer $\left(\mathrm{M}_{\mathrm{t}}\right)$ und jeweils ein Enantiomer des gauche-Monomers $\left(\mathrm{M}_{\mathrm{g}}\right)$ und des stabilsten Dimers $\left(\mathrm{D}^{\mathrm{gg}}\right)$ aus zwei gauche-Molekülen.

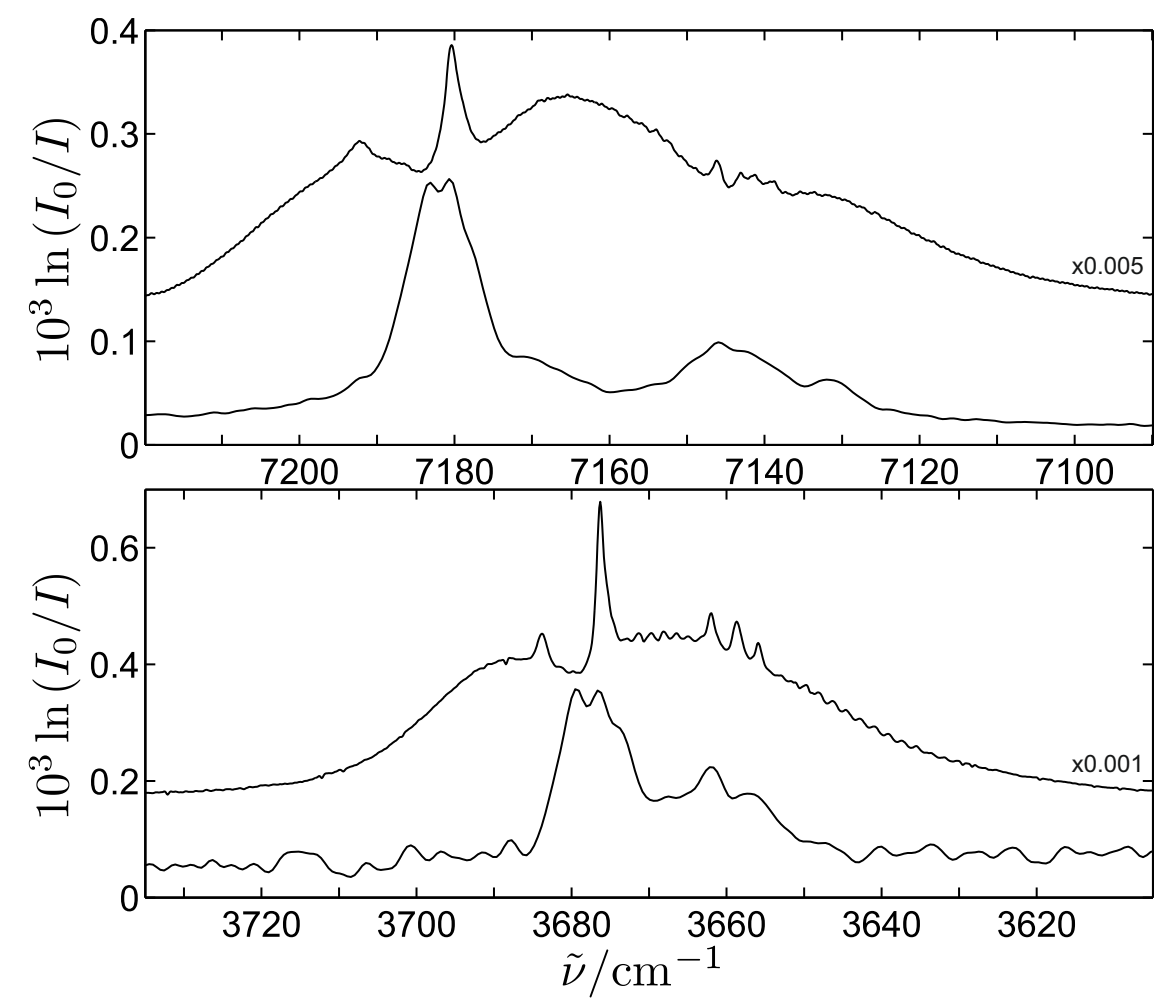

Abbildung 4.13.: Spektren der O-H Streckschwingung von Ethanol: Gasphase bei Raumtemperatur (jeweils oben) und Jetexpansionen (jeweils unten; Fundamentale 0.3 bar Helium, 0.1\% Ethanol; Oberton: 0.9 bar Helium, 0.3\% Helium).

men. Dazu wurden 10 mbar Ethanol und 1 bar Stickstoff in eine $23 \mathrm{~cm}$ lange Gaszelle gefüllt. Die Jetspektren sind in Abbildung 4.13 bei unterschiedlichen Bedingungen gezeigt. Das Fundamentalspektrum entspricht mit $0.1 \%$ Ethanol in 0.3 bar Helium einer deutlich geringeren Konzentration als das Obertonspektrum mit $0.3 \%$ Ethanol in 0.9 bar Helium. Dadurch liegen unter den Bedingungen des Fundamentalspektrums deutlich weniger Cluster vor, was die Überlappung der Monomerbanden durch Akzeptorbanden der Dimere verringert. Unter den Bedingungen des Obertonspektrums ist allerdings die Dimerkonzentration maximiert, sodass hier ein großer Anteil 
von Akzeptorintensität im Bereich der Monomerbanden vorliegt.

Die Bandenzentren der O-H Streckschwingung von trans-Ethanol lassen sich am besten anhand der Spektren der Jetexpansionen zuordnen. In Abbildung 4.13 ist die Rotationsstruktur der Jet-gekühlten Banden bei $2 \mathrm{~cm}^{-1}$ Auflösung gerade noch erkennbar. Die Bandenzentren lassen sich bei 3676.6(2) $\mathrm{cm}^{-1}$ und 7180.6(2) $\mathrm{cm}^{-1}$ festlegen. Sie liegen damit in Jetexpansionen um etwa $0.1-0.3 \mathrm{~cm}^{-1}$ blauverschoben gegenüber den Spitzen der scharfen Q-Zweige in den Gasphasenspektren bei Raumtemperatur, was sich als Temperatureffekt erklären lässt. Es ergibt sich eine Anharmonizitätskonstante von $-86.3(2) \mathrm{cm}^{-1}$ für trans-Ethanol. Publiziert wurde bisher ein Wert von $-85.0 \mathrm{~cm}^{-1}$ aus einem Birge-Sponer Fit an die Bandenpositionen von Fundamentale bis vierten Oberton, ${ }^{122}$ deren Abweichung möglicherweise auf die Wirkung höherer Anharmonizitätskonstanten bei den Übergängen zu hohen Quantenzahlen zurückgeht.

Die Auswirkung der Tunneldynamik auf das Absorptionsspektrum der O-H Streckschwingung von gauche-Ethanol wurde, soweit bekannt, in der Literatur noch nicht analysiert. Drei prominente Peaks kennzeichnen den Fundamentalbereich des gasförmigen gauche Monomers bei Raumtemperatur, bei dem außerdem eine reichhaltige Feinstruktur durch Rotation oder heiße Übergänge $\mathrm{zu}$ finden ist (siehe Abbildung 4.13). Die drei erwähnten Peaks liegen bei $3662.0(1) \mathrm{cm}^{-1}, 3658.7(1) \mathrm{cm}^{-1}$ und $3655.9(1) \mathrm{cm}^{-1}$. Sie sollen hier im Folgenden rein empirisch interpretiert werden. Die Schwingungsniveaus von gauche-Ethanol werden durch die Tunneldynamik in zwei Zustände aufgespalten, deren Differenz im Grundzustand $3.3 \mathrm{~cm}^{-1}$ beträgt. ${ }^{178}$ Der Übergang mit der höchsten Wellenzahl der Fundamentale muss demnach vom unteren (u) Tunnelniveau des Grundzustands in das obere Tunnelniveau (o) des $\mathrm{O}-\mathrm{H}$ Streckschwingung erfolgen. Demnach kann der Peak bei 3662.0(1) $\mathrm{cm}^{-1}(\mathrm{u}) \rightarrow(\mathrm{o})$ zugeordnet werden, während demzufolge der (o) $\rightarrow$ (u) Übergang dem Peak mit der geringsten Energie bei $3655.9(1) \mathrm{cm}^{-1}$ entspricht. Aus der bekannten Aufspaltung des Schwingungsgrundzustands ergeben sich damit erwartete Wellenzahlen für (o) $\rightarrow$ (o) von ungefähr 3658.7 und für (u) $\rightarrow$ (u) von ungefähr $3659.2 \mathrm{~cm}^{-1}$. Beide könnten im beobachteten Peak bei 3658.7(1) überlappen. Dieser Peak wurde auch in Ramanspektren von gasförmigem gauche-Ethanol bei $3660 \mathrm{~cm}^{-1}$ gefunden und ebenfalls den Übergängen $(\mathrm{o}) \rightarrow(\mathrm{o})$ und $(\mathrm{u}) \rightarrow(\mathrm{u})$ zugeordnet. ${ }^{59}$ Aus der hier vorgenommenen Interpretation der drei Peaks ergibt sich für den angeregten $\mathrm{O}-\mathrm{H}$ Streckschwingungszustand von gauche-Ethanol eine Tunnelaufspaltung von 2.8(1) $\mathrm{cm}^{-1}$. Das Bandenzentrum des Fundamentalübergangs ließe sich bei $3659.0(1) \mathrm{cm}^{-1}$ annehmen. Allerdings könnten die Peaks, die hier als Q-Zweige interpretiert werden, wie im Falle von trans-Ethanol in der Raumtemperatur Gasphase leicht rotverschoben sein. Somit ist es sicherer das Bandenzentrum im Bereich von 3659.3(4) anzunehmen. Das Jetspektrum kann hier nicht zur eindeutigen Belegung eines Temperatureffekts herangezogen werden. Es zeigt in Abbildung 4.13 einen Peak bei 3662.1(6) der dem (u) $\rightarrow$ (o) Übergang entspricht. Weitere Peaks lassen sich auf Grund der geringen Auflösung und eventueller Überlappung mit Dimerakzeptorbanden im Jetspektrum jedoch nicht erkennen.

Die Interpretation des Obertonbereichs kann analog zur Fundamentale versucht werden. In Abbildung 4.13 zeigt das Gasphasenspektrum von gauche-Ethanol vier schwache Peaks: 7146.2(2) $\mathrm{cm}^{-1}, 7143.2(1) \mathrm{cm}^{-1}, 7141.5(3) \mathrm{cm}^{-1}$ und 7139.0(4) $\mathrm{cm}^{-1}$. Der Peak mit der höchsten Wellenzahl von 7146.2(2) $\mathrm{cm}^{-1}$ ist gut von den anderen separiert und kann wieder dem (u) $\rightarrow$ (o) Übergang zugeordnet werden. An dieser Position zeigt sich auch die höchste Intensität des Jetspektrums bei 7146.0(5) $\mathrm{cm}^{-1}$. Die anderen Peaks im Gasphasenspektrum überlappen 


\section{4. $O-H$ Streckschwingungen}

partiell, jedoch lässt sich auf Grund der Grundzustandsaufspaltung ${ }^{178}$ von $3.3 \mathrm{~cm}^{-1}$ der Peak bei 7143.2(1) dem (o) $\rightarrow$ (o) Übergang zuweisen. Der Peak bei der niedrigsten Wellenzahl von $7139.0(4) \mathrm{cm}^{-1}$ sollte dem (o) $\rightarrow$ (u) Übergang entsprechen. Aus der Aufspaltung des Grundzustands ergibt sich eine erwartete Wellenzahl für $(\mathrm{u}) \rightarrow(\mathrm{u})$ von $7142.3 \mathrm{~cm}^{-1}$, was nur schlecht mit dem tatsächlich beobachteten Peak bei $7141.5(3) \mathrm{cm}^{-1}$ übereinstimmt. Diese Interpretation der vier Peaks im Obertonbereich fällt damit weit weniger befriedigend aus als die Analyse der Fundamentale, was an der schlechteren Übereinstimmung der beobachteten Wellenzahlen mit der Grundzustandsaufspaltung liegt. Aus der hier präsentierten Analyse ergibt sich eine erhöhte Tunnelaufspaltung des zweiten angeregten $\mathrm{O}-\mathrm{H}$ Streckschwingungszustands von gaucheEthanol im Bereich von 4.0(3) $\mathrm{cm}^{-1}$. Diese Erhöhung wäre zwar unerwartet, aber möglich im Falle von Resonanzen mit anderen Zuständen, die mitunter auch als Erklärung für die "verzerrten" Tunnelniveaus des zweiten angeregten Zustands herhalten könnten. Es ergibt sich aus den gemessenen Peaks ein Bandenzentrum für den Oberton von $7142.9(7) \mathrm{cm}^{-1}$, bei dem wie bei trans-Ethanol eventuelle Temperatureffekte berücksichtigt wurden. Daraus lässt sich zusammen mit dem Bandenzentrum der Fundamentale eine Anharmonizitätskonstante von $-87.9(5) \mathrm{cm}^{-1}$ für die $\mathrm{O}-\mathrm{H}$ Streckschwingung von gauche-Ethanol ermitteln. Sie fällt höher aus als die Anharmonizität von trans-Ethanol, was auch in der Literatur bei der Mittelung über mehrere Obertöne gefunden wurde $\left(-86 \mathrm{~cm}^{-1}\right.$ für gauche-Ethanol). ${ }^{122}$

In Jetexpansionen von Ethanol in Helium liegen mindestens vier unterscheidbare Ethanoldimere vor. ${ }^{59,179}$ Diese Vielfalt ist auf die neun Kombinationsmöglichkeiten der Ethanolkonformere miteinander zurückzuführen. ${ }^{180,181}$ Durch Zumischen von Argon konnten die Moleküle im stabilsten Dimer als gauche-Donor und gauche-Akzeptor $\left(D^{g g}\right)$ mit gleicher Chiralität identifiziert werden ${ }^{59,181}$ (Vgl. Abb. 4.12). Das zweitstabilste Dimer wurde in Neonexpansionen identifiziert und besteht aus gauche-Donor und trans-Akzeptor $\left(\mathrm{D}^{\mathrm{gt}}\right) .{ }^{59}$ Die genaue Geometrie des zweitstabilsten Dimers ist jedoch noch nicht endgültig aufgeklärt, da hierfür zwei unterschiedliche Strukturen in Frage kommen. ${ }^{59,182}$ Die Jetspektren in Abbildung 4.14 zeigen auch den Dimer- und Trimerbereich von O-H Streckschwingungsfundamentale und -oberton. ${ }^{11}$ Die drei intensiven Fundamentalsignale im Dimerdonorbereich lassen sich gemäß der Literatur $^{59,181}$ wie folgt zuordnen: Die Bande mit der stärksten Verschiebung zum gauche-Monomer von $-127.8(4) \mathrm{cm}^{-1}$ bei 3531.5(2) $\mathrm{cm}^{-1}$ entspricht $\mathrm{D}^{\mathrm{gg}}$, während $\mathrm{D}^{\mathrm{gt}}$ der Bande mit höchster Wellenzahl bei 3547.1(3) $\mathrm{cm}^{-1}$ zu Grunde liegt. Dazwischen liegt ein Signal bei 3539.4(3) $\mathrm{cm}^{-1}$ bei dem vermutlich zwei Dimere überlagern. Da das Obertonspektrum von Ethanol in Heliumexpansionen in Abbildung 4.14 selbst nach Mittelung über mehr als 3000 Pulse kaum Signal von Dimerdonoren zeigt, wurde den Expansionen 1.5\% Argon hinzugefügt um die Konzentration des stabilsten Dimers zu erhöhen und wenigstens dieses im Obertonspektrum sichtbar zu machen. Tatsächlich verdoppelt sich dadurch die Konzentration von $D^{\text {gg }}$ etwa, was die Detektion des schwachen Obertonsignals seines Donors bei $6860.9(7) \mathrm{cm}^{-1}$ ermöglicht. Daraus ergibt sich eine Anharmonizitätskonstante von -101.1(4) $\mathrm{cm}^{-1}$, also eine Erhöhung der Anharmonizität gegenüber dem gauche-Monomer um 15.0(8)\%. Die Donorsignale in den Spektren mit ausschließlich Helium als Trägergas sind kaum vom Rauschen zu unterscheiden. Man könnte jedoch mit viel Optimismus und unter ausdrücklichem Verweis auf das schlechte Signal-zu-Rauschen Verhältnis und der damit großen statistischen Unsicherheit der Daten auch Banden in den Spektren der Heliumexpansionen zuordnen. Die Wellenzahlen der Bandenzentren sind in Tabelle 4.7 


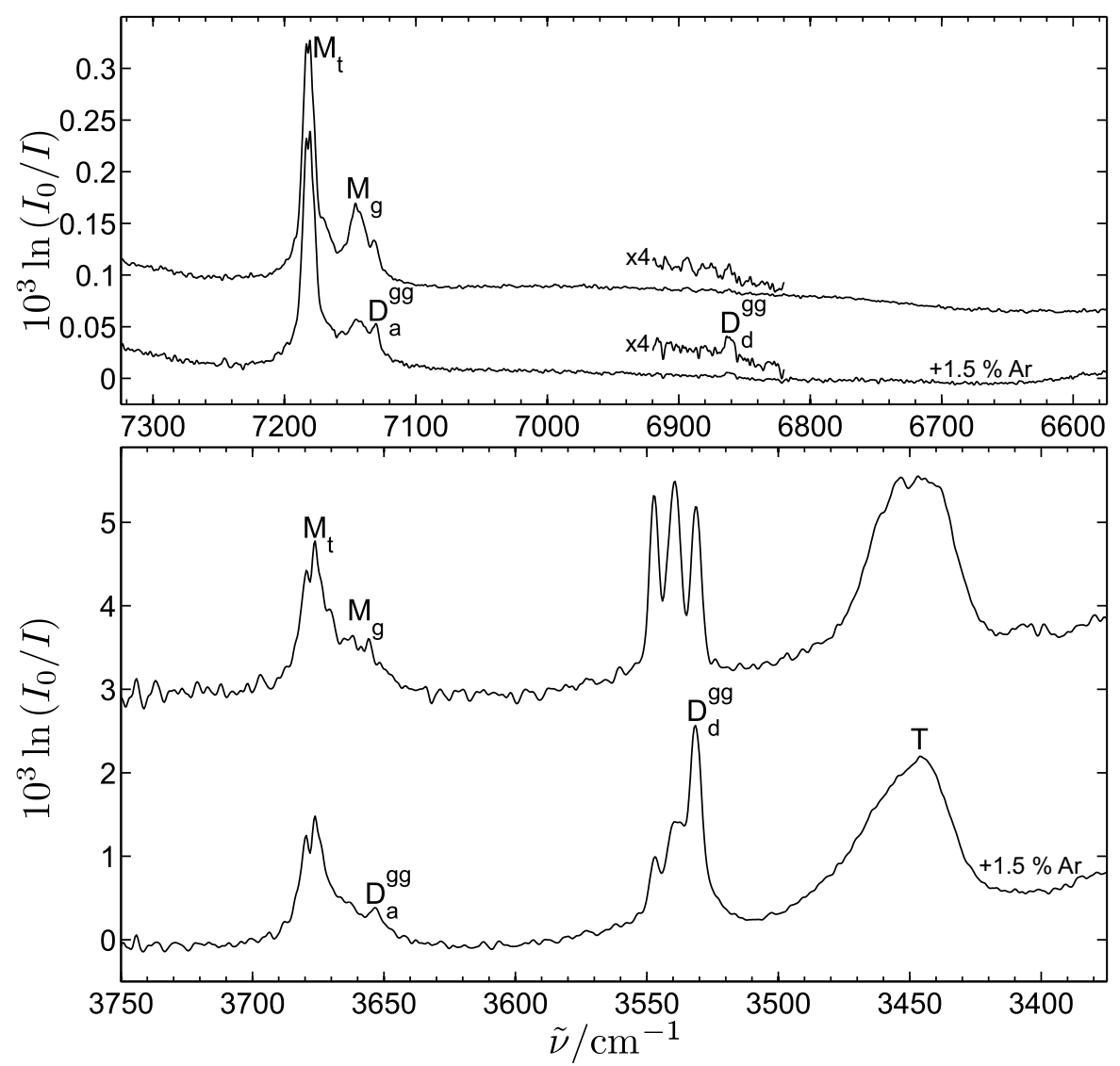

Abbildung 4.14.: Jetspektren von 0.3\% Ethanol in 0.9 bar Expansionen im Fundamentalbereich (oberes Spektrum: reines Helium als Trägergas, 100 Pulse; unteres Spektrum: Helium $+1.5 \%$ Argon, 200 Pulse) und im Obertonbereich (oberes Spektrum: reines Helium, 3450 Pulse; unteres Spektrum: Helium $+1.5 \%$ Argon, 1600 Pulse). Gekennzeichnet sind die Banden des trans-Monomer $\mathrm{M}_{\mathrm{t}}$, gaucheMonomer $\mathrm{M}_{\mathrm{g}}$, sowie die Banden des Akzepors $\mathrm{D}_{\mathrm{a}}^{\mathrm{gg}}$ und Donors $\mathrm{D}_{\mathrm{d}}^{\mathrm{gg}}$ des stabilsten Dimers und der Trimere T. Durch das zusätzliche Argon in den jeweils unteren Spektren relaxieren die Dimere vermehrt zur stabilsten Struktur.

gegeben. Basierend auf den Messungen der Heliumexpansionen erhöht sich der Fehler der Anharmonizität des Donors von $D^{\text {gg }}$ auf $-101(1) \mathrm{cm}^{-1}$. Dem zweitstabilsten Dimer $D^{\text {gt }}$ ließe sich eine Donoranharmonizität von $-100.2(6) \mathrm{cm}^{-1}$ zuordnen. Die überlappenden Donorbanden der weniger stabilen Dimere wären bei 6879(3) anzutreffen.

Die Akzeptorbanden der Dimere überlappen in den Jetexpansionen mit den Signalen der Monomere, jedoch lassen sie sich mit Hilfe der Literatur ${ }^{59}$ und Vergleich der Spektren von Expansionen mit und ohne zusätzlichem Argon zuordnen. Die Akzeptorfundamentale des stabilsten Dimers $D^{g g}$ wurde mittels ihres vergleichsweise leicht detektierbaren Ramansignals in Jetexpansionen bei $3654 \mathrm{~cm}^{-1}$ gefunden. ${ }^{59}$ In den Filet-Jetspektren tritt ihr Signal in den Expansionen mit zusätzlichem Argon auf Grund der geringeren gauche-Monomerkonzentration besonders 


\section{4. $O-H$ Streckschwingungen}

Tabelle 4.7.: Spektroskopische Daten aus Ethanolmessungen: Bandenzentren (in $\mathrm{cm}^{-1}$ ) der $\mathrm{O}-\mathrm{H}$ Streckschwingung des trans- $\left(\mathrm{M}_{\mathrm{t}}\right)$ und gauche-Monomers $\left(\mathrm{M}_{\mathrm{g}}\right)$, der Dimerakzeptoren $\left(\mathrm{D}_{\mathrm{a}}\right)$ und der Dimerdonoren $\left(\mathrm{D}_{\mathrm{d}}\right)$ des stabilsten Dimers (gauche-gauche $D^{g g}$ ) und zweitstabilsten Dimers (gauche-trans $D^{g t}$ ). Die integrierten Absorbanzen (in $\mathrm{cm}^{-1}$ ) der Banden sind kursiv gedruckt. Bei der Berechnung der Fundamentalezu-Oberton Verhältnisse, müssen die integrierten Absorbanzen der Fundamentalen mit dem Korrekturfaktor von 0.78(13) multipliziert werden. Die Werte in eckigen Klammern sind mit einer hohen experimentellen Unsicherheit belastet.

\begin{tabular}{|c|c|c|c|c|c|c|c|}
\hline Gasphase & $\begin{array}{l}\nu_{\mathrm{OH}} \\
2 \nu_{\mathrm{OH}}\end{array}$ & $\begin{array}{l}\mathrm{M}_{\mathrm{t}} \\
3676.3(2) \\
7180.5(2)\end{array}$ & $\begin{array}{l}\mathrm{M}_{\mathrm{g}} \\
3662.0(1) \\
3658.7(1) \\
3655.9(1) \\
7146.2(2) \\
7143.1(1) \\
7141.5(3) \\
7139.0(4)\end{array}$ & $\mathrm{D}_{\mathrm{a}}^{\mathrm{gt}}$ & $\mathrm{D}_{\mathrm{a}}^{\mathrm{gg}}$ & $\mathrm{D}_{\mathrm{d}}^{\text {gt }}$ & $\mathrm{D}_{\mathrm{d}}^{\mathrm{gg}}$ \\
\hline He Expansion & $\begin{array}{l}\nu_{\mathrm{OH}} \\
2 \nu_{\mathrm{OH}}\end{array}$ & $\begin{array}{l}3676.6(2) \\
8(1) \\
7180.6(2) \\
1.3(3)\end{array}$ & & $\begin{array}{l}3670.4(4) \\
2.4(5) \\
7170(2) \\
0.24(9)\end{array}$ & & $\begin{array}{l}3547.1(3) \\
4.4(3) \\
{[6894(1)]} \\
{[0.008(3)]}\end{array}$ & $\begin{array}{l}3531.2(3) \\
4.2(3) \\
{[6861.2(9)]} \\
{[0.008(3)]}\end{array}$ \\
\hline He+Ar Expansion & $\begin{array}{l}\nu_{\mathrm{OH}} \\
2 \nu_{\mathrm{OH}}\end{array}$ & $\begin{array}{l}3676.3(3) \\
6.9(7) \\
7180.4(3) \\
1.2(2)\end{array}$ & & & $\begin{array}{l}3653.3(5) \\
1.8(3) \\
7130.3(7) \\
0.17(6)\end{array}$ & $\begin{array}{l}3546.9(2) \\
2.4(2)\end{array}$ & $\begin{array}{l}3531.5(2) \\
8.5(4) \\
6860.9(7) \\
0.016(3)\end{array}$ \\
\hline
\end{tabular}

hervor und kann bei $3653.3(5) \mathrm{cm}^{-1}$ lokalisiert werden. Der entsprechende Oberton liegt bei 7130.3(7) $\mathrm{cm}^{-1}$, sodass sich für die $\mathrm{O}-\mathrm{H}$ Streckschwingung des Akzeptors im stabilsten Dimer eine Anharmonizität von $-88.2(6) \mathrm{cm}^{-1}$ ergibt. Gegenüber dem Wert für gauche-Ethanol entspricht das einer leichten Erhöhung, jedoch sei hier nochmal auf die besondere Unsicherheit bei der Bestimmung der gauche-Monomer Anharmonizität durch eine möglicherweise unrichtige Interpretation des Obertonspektrums hingewiesen. Die O-H Streckschwingungsbanden des transAkzeptors treten als Schultern an der trans-Methanolbande auf und können für die Fundamentale bei 3670.4(4) $\mathrm{cm}^{-1}$ (Raman: ${ }^{59} 3672 \mathrm{~cm}^{-1}$ ) und den Oberton bei 7170(2) $\mathrm{cm}^{-1}$ gefunden werden. Die resultierende Anharmonizitätskonstante von $-87(1) \mathrm{cm}^{-1}$ bleibt innerhalb der Fehlergrenzen unverändert gegenüber dem trans-Monomer.

Für die Bestimmung der gemessenen Intensitäten wurden vor allem Anpassungen von miteinander gemischten Gauß- und Lorentzfunktionen an die Spektren vorgenommen, um der starken Überlappung der einzelnen Banden Rechnung zu tragen. Nur die Obertöne der Dimerdonorschwingungen wurden durch Integration quantifiziert. Die Daten sind gemeinsam mit den bestimmten Bandenzentren in Tabelle 4.7 zusammengefasst. Wieder ist das Fundamentale zu Oberton Verhältnis der Monomere in den Jetspektren mit 5(1) für trans- und 5(2) für gauche-Ethanol systematisch zu gering gegenüber einem Literaturwert von 7.5(4), ${ }^{116}$ für den über beide Konfor- 
mere gemittelt wurde. Die Intensitäten der Akzeptorbanden führen zu Fundamentale zu Oberton Verhältnissen von 6(3) für $\mathrm{D}^{\text {gg }}$ und 8(4) $\mathrm{D}^{\text {gt }}$, die innerhalb ihrer Fehlergrenzen mit dem gemittelten Monomerwert ${ }^{116}$ übereinstimmen. Für die Dimerdonorschwingung des stabilsten Dimers $\mathrm{D}^{\text {gg }}$ ergibt sich ein Verhältnis von 400(100) und damit eine Steigerung um 5000(1000)\% durch die Ausbildung der Wasserstoffbrücke gegenüber dem Monomerverhältnis. Aus den schwachen Signalen des Spektrums der Jetexpansion von Ethanol in reinem Helium kann ein Verhältnis von 400(200) für die Donorstreckschwingung des zweitstabilsten Dimers D ${ }^{\text {gt }}$ mit deutlich höherem Fehler abgeschätzt werden. Die geringen Intensitäten der Dimerdonorobertöne lassen es nicht verwunderlich erscheinen, dass keine Trimerobertöne beobachtet werden. Daher lässt sich für das Fundamentale-zu-Oberton Verhältnis der breiten Trimerbande nur eine Untergrenze von 800 angeben.

In Zukunft wäre es vor allem wünschenswert das $\mathrm{O}-\mathrm{H}$ Streckschwingungsspektrum von gauche-Ethanol genauer zu analysieren. Dies könnte neben rotationsaufgelöster Spektroskopie in der Gasphase auch am Filet-Jet geschehen, indem bei geringem Druck und hoher Monomerkonzentration analog zu dem Fundamentaljetspektrum in Abbildung 4.13 bei verbesserter Auflösung von 1 oder $0.5 \mathrm{~cm}^{-1}$ gemessen wird. So könnten zumindest die Übergänge aus dem unteren Tunnelniveau bei vereinfachter Rotationsstruktur besser sichtbar gemacht werden. Außerdem könnte eine Verringerung des Rauschens durch Optimierung der Messbedingungen (beispielsweise durch empfindlichere Detektoren und hellere Strahlungsquellen) die Detektion der Dimerdonorobertöne in Heliumexpansionen verbessern und so die subtilen Unterschiede der anharmonischen Effekte durch die Wasserstoffbrücke in den verschiedenen Dimerkonformationen aufzeigen.

\subsection{4. tert-Butylalkohol}

Das Fundamentalspektrum von tert-Butylalkohol in Jetexpansionen wurde bereits hinsichtlich seiner Clustersignale analysiert. ${ }^{37}$ In Abbildung 4.15 sind die Oberton- und Fundamentalspektren von tert-Butylalkohol in Überschallexpansionen gezeigt. ${ }^{11}$ Die Rotationsstruktur der Monomerübergänge ist bei $2 \mathrm{~cm}^{-1}$ Auflösung gerade noch erkennbar. Ihre Bandenzentren lassen sich auf Grund dieser Vereinfachung leicht bestimmen und werden bei $3642.3(2) \mathrm{cm}^{-1}$ und $7110.6(2) \mathrm{cm}^{-1}$ festgelegt. Die daraus resultierende diagonale Anharmonizitätskonstante für die $\mathrm{O}-\mathrm{H}$ Streckschwingung des Monomers von $-87.0(2) \mathrm{cm}^{-1}$ liegt etwas über dem Wert aus einer Birge-Sponer Auftragung von $86.0(8) \mathrm{cm}^{-1}$ aus den Bandenzentren der Fundamentale bis zum fünften Oberton, ${ }^{124}$ was an der Wirkung höherer Anharmonizitätskonstanten in den hoch angeregten Streckschwingungszuständen liegen könnte. In den Jetspektren wird die Intensität des Obertonübergangs gegenüber der Fundamentale um einen systematisch zu kleinen Faktor 4(1) abgeschwächt; der vertrauenswürdigere Gasphasenwert liegt bei 6.8(8). ${ }^{116}$ Die $\mathrm{O}-\mathrm{H}$ Streckschwingungsübergänge des Dimerakzeptors sind in den Jetspektren gut separiert und lassen sich daher einfach auswerten. Im Vergleich zu den Monomersignalen zeigen die Akzeptorbanden eine leichte Rotverschiebung und ergeben eine etwas erhöhte Anharmonizitätskonstante von $87.9(3) \mathrm{cm}^{-1}$, sowie ein höheres Fundamentale-zu-Oberton Intensitätsverhältnis von 10(3). Somit kann am Beispiel von tert-Butylalkohol gezeigt werden, dass der Einfluss der Wasserstoffbrücke auf die Streckschwingung der O-H Akzeptorgruppe im Vergleich zum Donor nur gering 


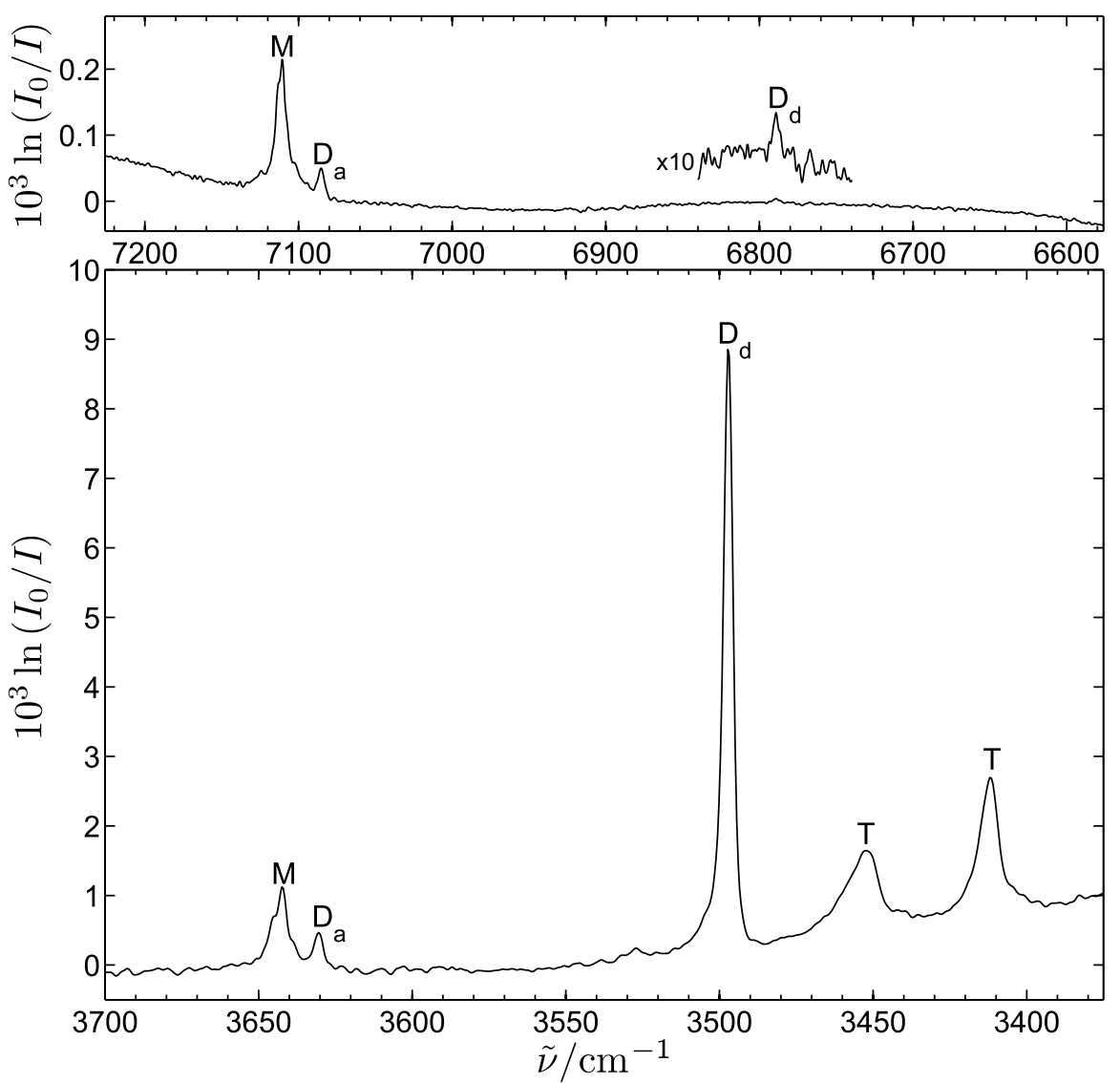

Abbildung 4.15.: Oberton- (oben, 1350 Pulse) und Fundamentalspektrum (unten, 200 Pulse) der Jetexpansionen von $0.3 \%$ tert-Butylalkohol in 0.8 bar Helium im Bereich der $\mathrm{O}-\mathrm{H}$ Streckschwingung. Markiert sind die Banden von Monomer (M), Dimerakzeptor $\mathrm{D}_{\mathrm{a}}$ und -donor $\mathrm{D}_{\mathrm{d}}$, sowie Trimer $(\mathrm{T})$. Die Strukturen der Cluster sind in Abbildung 2.1 in Kapitel 2.2 abgebildet.

ausfällt. Die Fundamentalschwingung des Dimerdonors ist bei $3497.1(3) \mathrm{cm}^{-1} \mathrm{zu}$ finden und ist damit um -145.2(4) $\mathrm{cm}^{-1}$ zum Monomer verschoben. Aus der Analyse des äußerst schwachen Obertons bei 6789.1(4) $\mathrm{cm}^{-1}$ ergibt sich eine Anharmonizitätskonstante von -102.6(4) $\mathrm{cm}^{-1}$ für die Streckschwingung des Dimerdonors, die damit um 17.9(5)\% größer ausfällt als im Monomer. Für das Fundamentale zu Oberton Intensitätsverhältnis kann ein Bereich von 1000(400) angegeben werden, was einer Steigerung um 15000(6000)\% entspricht. Wieder können keine Obertöne oder Kombinationen der Trimerstreckschwingungen beobachtet werden. Diese Signale müssen daher mindestens um einen Faktor 800 schwächer sein als die intensivste Trimerfundamentale. In Tabelle 4.8 sind die spektroskopischen Daten der Fundamentalübergänge und Obertöne vom tert-Butylalkoholmonomer und -dimer zusammengefasst. 
Tabelle 4.8.: Spektroskopische Daten aus Jetexpansionen von tert-Butylalkohol: Bandenzentren der $\mathrm{O}-\mathrm{H}$ Streckschwingung des Monomers $(\mathrm{M})$, Dimerakzeptors $\left(\mathrm{D}_{\mathrm{a}}\right)$ und Dimerdonors $\left(\mathrm{D}_{\mathrm{d}}\right)$ in $\mathrm{cm}^{-1}$ und integrierte Absorbanzen $\int 10^{3} \ln \left(\frac{I_{0}}{I}\right) \mathrm{d} \tilde{\nu}$ in $\mathrm{cm}^{-1}$ (kursiv gedruckt) der Banden. Bei der Berechnung der Fundamentale-zu-Oberton Verhältnisse, müssen die integrierten Absorbanzen der Fundamentalen mit dem Korrekturfaktor von $0.78(13)$ multipliziert werden.

\begin{tabular}{llll}
\hline & $\mathrm{M}$ & $\mathrm{D}_{\mathrm{a}}$ & $\mathrm{D}_{\mathrm{d}}$ \\
\hline$\nu_{\mathrm{OH}}$ & $3642.3(2)$ & $3630.4(2)$ & $3497.1(3)$ \\
& $4.15(9)$ & $1.3(1)$ & $16.9(6)$ \\
$2 \nu_{\mathrm{OH}}$ & $7110.6(2)$ & $7085.1(4)$ & $6789.1(4)$ \\
& $0.9(2)$ & $0.11(2)$ & $0.013(4)$ \\
\hline
\end{tabular}
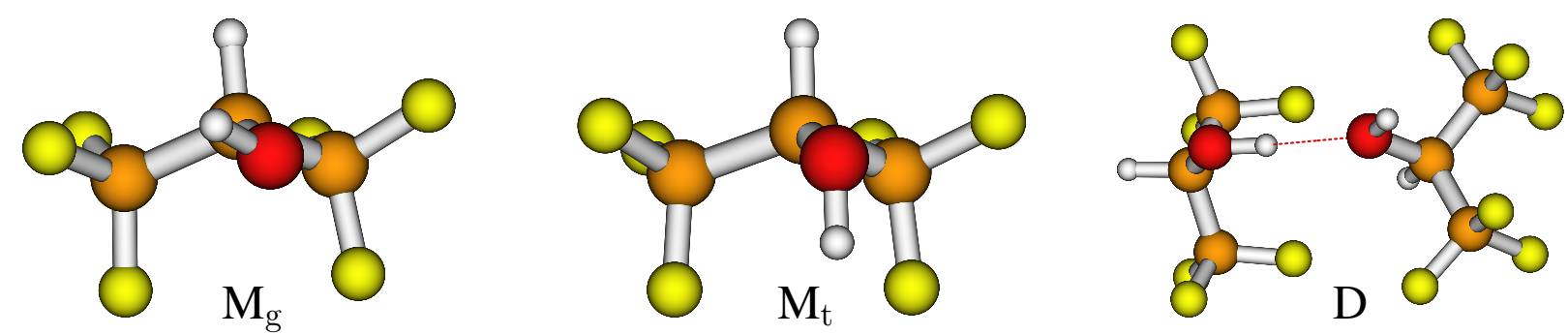

Abbildung 4.16.: Optimierte Strukturen (B3LYP-D3/6-311+G(2d,p)) eines Enantiomer des gauche-Monomers $\left(\mathrm{M}_{\mathrm{g}}\right)$ und des stabileren trans-Monomers $\left(\mathrm{M}_{\mathrm{t}}\right)$ von Hexafluorisopropanol, sowie des stabilsten Dimers (D) aus zwei trans-Molekülen

\subsubsection{Hexafluorisopropanol}

Hexafluorisopropanol (1,1,1,3,3,3-Hexafluor-2-propanol) liegt in der Gasphase in zwei Konformationen vor: ${ }^{183}$ dem stabileren trans-Konformer $\left(M_{t}\right)$ und der zweifach entarteten gauche Konformation $\left(\mathrm{M}_{\mathrm{g}}\right)$ (Vgl. Abb. 4.16). Trotz der energetisch äquivalenten Minima der gauche Konformation, ${ }^{184,185}$ wurden in der Literatur soweit bekannt bisher keine Effekte durch eine mögliche Tunnelaufspaltung ihrer Schwingungszustände beschrieben. Die O-H Streckschwingungsübergänge bis zum zweiten Oberton von Hexafluorisopropanol in der Gasphase sind in Abbildung 4.17 gezeigt. Die Wellenzahlen der Bandenmitten der beobachteten Übergänge sind in Tabelle 4.9 zusammengefasst. Die Fundamentalwellenzahlen von $3626 \mathrm{~cm}^{-1}$ für das transMonomer und $3667 \mathrm{~cm}^{-1}$ für die gauche-Form stimmen mit den Literaturdaten überein. ${ }^{47,183,184,186,187}$ Die ersten Obertöne können bei $7085 \mathrm{~cm}^{-1}$ und $7166 \mathrm{~cm}^{-1}$ beobachtet werden, ${ }^{47}$ wurden jedoch in einer älteren Studie bei höheren Wellenzahlen angegeben. ${ }^{187} \mathrm{Zu}$ sammen mit den zweiten Obertönen ergeben sich aus einer Birge-Sponer Auftragung Anharmonizitätskonstanten von $-82 \mathrm{~cm}^{-1}$ für trans- und $-83 \mathrm{~cm}^{-1}$ für gauche-Hexafluorisopropanol aus diesen Gasphasendaten.

In Jetexpansionen von Hexafluorisopropanol relaxiert das gauche-Monomer fast vollständig zur trans-Form. ${ }^{47,184}$ Die Bandenzentren für trans-Hexafluorisopropanol, die sich aus den Jetspektren (Abbildung 4.18) bestimmen lassen, sind deutlich rotverschoben gegenüber den Wel- 

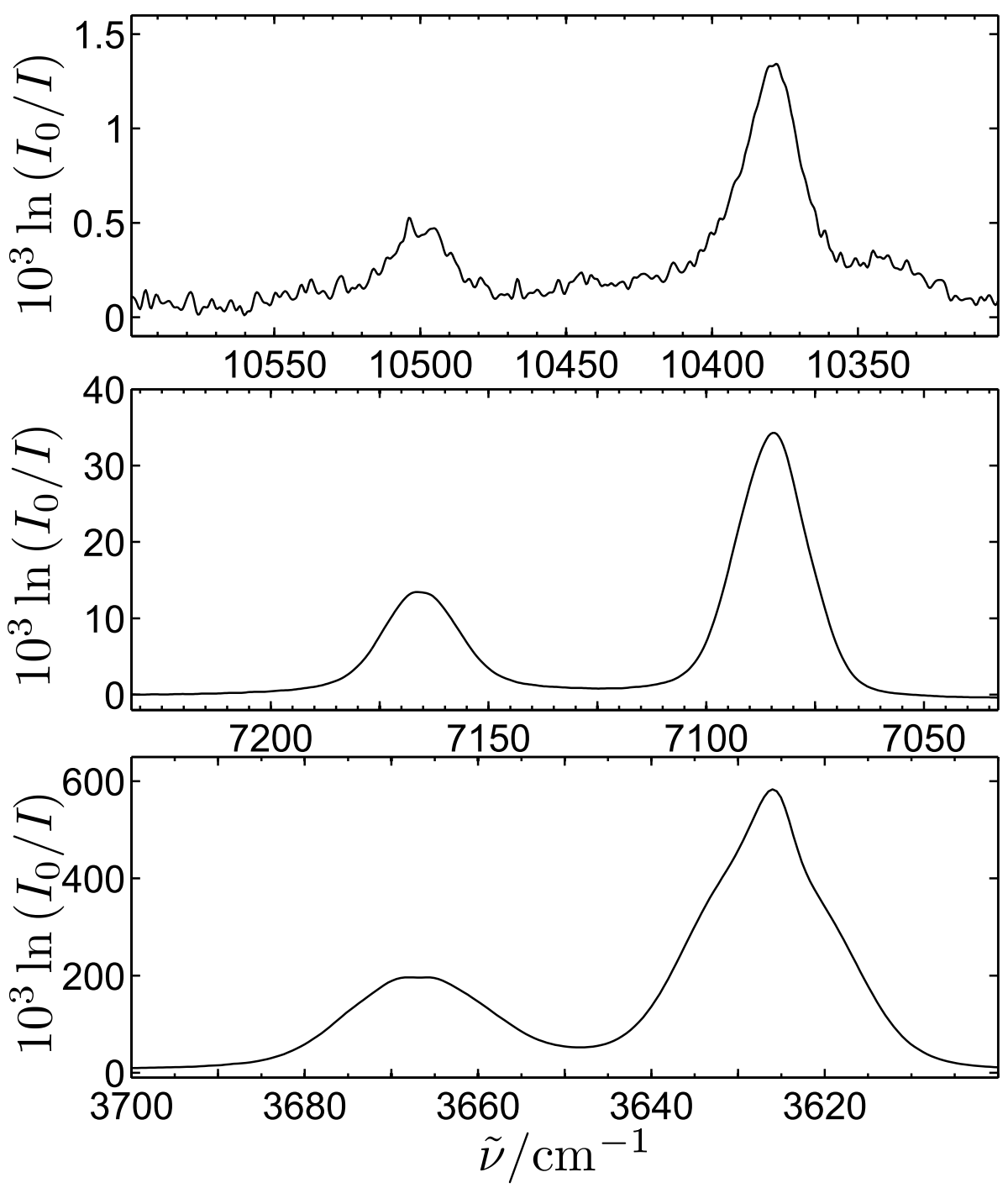

Abbildung 4.17.: Die hier gezeigten Gasphasenspektren der Fundamentale, sowie des ersten und zweiten Obertons von Hexafluorisopropanol wurden am Filet-Jet aufgenommen. Den Banden bei jeweils höherer Energie der Dubletts ist das gaucheKonformer zuzuordnen. Die Banden des trans-Monomers liegen jeweils bei niedrigerer Wellenzahl.

lenzahlen der warmen Gasphase. Sie liegen bei 3623.6(4) $\mathrm{cm}^{-1}$ für die Fundamentale und bei $7078.9(4) \mathrm{cm}^{-1}$ für den ersten Oberton der O-H Streckschwingung. Das führt auch zu einer höheren Anharmonizitätskonstante von $-84.2(4) \mathrm{cm}^{-1}$. Auf Grund der relativ hohen Fundamentalintensität des Monomers ${ }^{184}$ ist der Wert seines Fundamentale-zu-Obertonverhältnisses von 16(4) aus den Jetspektren möglicherweise noch stärker systematisch fehlerbehaftet als im Fall der anderen Alkohole dieses Kapitels. Wahrscheinlich liegt es in Wahrheit bis zu einem Faktor 2 höher. Die Auswertung der Intensitäten in den Gasphasenspektren, auf die hier verzichtet werden soll, 
wäre von den Sättigungseffekten noch stärker betroffen, da hier die Absorbanz der Monomere um zwei Größenordnungen höher ausfällt und nicht ausreichend Stoßgas zur Linienverbreiterung vorhanden ist.

Tabelle 4.9.: Spektroskopische Daten für Hexafluorisopropanol: Bandenmitten aus Gasphasenmessungen und Bandenzentren aus Jetmessungen der $\mathrm{O}-\mathrm{H}$ Streckschwingung (in $\left.\mathrm{cm}^{-1}\right)$ des gauche- $\left(\mathrm{M}_{\mathrm{g}}\right)$ und trans-Monomers $\left(\mathrm{M}_{\mathrm{t}}\right)$, des Dimerakzeptors $\left(\mathrm{D}_{\mathrm{a}}\right)$ und des Dimerdonors $\left(\mathrm{D}_{\mathrm{d}}\right)$. Die integrierten Absorbanzen (in $\mathrm{cm}^{-1}$ ) der Banden sind kursiv gedruckt und wurden durch Bandenanpassungen von gemischten Gauß- und Lorentzfunktionen an das Spektrum ermittelt (außer dem Dimerdonoroberton, der mittels Integration quantifiziert wurde). Bei der Berechnung der Fundamentale-zuOberton Verhältnisse, müssen die integrierten Absorbanzen der Fundamentalen mit dem Korrekturfaktor von 0.78(13) multipliziert werden.

\begin{tabular}{llllll}
\hline & & $\mathrm{M}_{\mathrm{g}}$ & $\mathrm{M}_{\mathrm{t}}$ & $\mathrm{D}_{\mathrm{a}}$ & $\mathrm{D}_{\mathrm{d}}$ \\
\hline Gasphase & $\nu_{\mathrm{OH}}$ & 3667 & 3626 & & \\
& $2 \nu_{\mathrm{OH}}$ & 7166 & 7085 & & \\
& $3 \nu_{\mathrm{OH}}$ & 10500 & 10380 & & \\
He-Expansion & $\nu_{\mathrm{OH}}$ & & $3623.6(4)$ & $3616.6(4)$ & $3491.9(3)$ \\
& & & $59(5)$ & $16(4)$ & $98(5)$ \\
& $2 \nu_{\mathrm{OH}}$ & & $7078.9(4)$ & $7066.4(4)$ & $6786.6(6)$ \\
& & & $2.9(4)$ & $0.5(1)$ & $0.13(3)$ \\
\hline
\end{tabular}

In Abbildung 4.18 sind die Spektren der O-H Streckschwingung von Hexafluorisopropanol in Jetexpansionen dargestellt. ${ }^{7}$ Das Dimer ist in Form seiner Akzeptor- und Donorbanden gut zu erkennen. Es besteht aus zwei trans-Monomeren ${ }^{47}$ (Abb.4.16), weshalb für den Vergleich von Dimer mit Monomer eine genaue experimentelle Charakterisierung des gauche-Monomers nicht notwendig ist. Die Wellenzahlen und Intensitäten der Dimerbanden sind in Tabelle $4.9 \mathrm{zu}-$ sammen mit den Monomerdaten angegeben. Die Dimerakzeptorbanden sind leicht rotverschoben zum Monomer und ergeben eine Anharmonizitätskonstante von $-83.4(4) \mathrm{cm}^{-1}$, die damit niedriger ausfällt als beim Monomer. In wie weit das Fundamentale-zu-Oberton Verhältnis von 30(10) des Dimerakzeptors vom Monomerwert abweicht, kann hier nicht geklärt werden. Die Obertöne der Monomere und des Dimerakzeptors konnten bereits zuvor in Jetexpansionen nachgewiesen werden. ${ }^{47}$ Jedoch konnte die Detektion des Dimerdonorobertons erst mit der im Zuge dieser Arbeit erreichten Verbesserung der Messsensitivität erreicht werden. Die Dimerdonorbande ist im Obertonspektrum deutlich bei gutem Signal-zu-Rauschen Verhältnis zu erkennen. Aus ihr kann zusammen mit der Wellenzahl des Fundamentalübergangs eine Anharmonizitätskonstante von $-98.6(4) \mathrm{cm}^{-1}$ erhalten werden - eine Steigerung von 17.1(7)\% gegenüber dem Monomer. Das Intensitätsverhältnis von Fundamentale zu Oberton beträgt für die Dimerdonorschwingung 600(200) und fällt damit eine Größenordnung höher aus als in den freien O-H Oszillatoren des Monomers und Dimers. Neben dem sehr intensiven Signal der Dimerdonorschwingung, das um $-131.7(5) \mathrm{cm}^{-1}$ zum Monomer verschoben ist, sind im Fundamentalspektrum einige schwächere Banden zu beobachten. Das Signal bei $3560 \mathrm{~cm}^{-1}$ könnte auf einen gauche-Donor in einem gauche-gauche Dimer zurückzuführen sein. Die Banden bei $3505 \mathrm{~cm}^{-1}$ und $3466 \mathrm{~cm}^{-1}$ lassen 

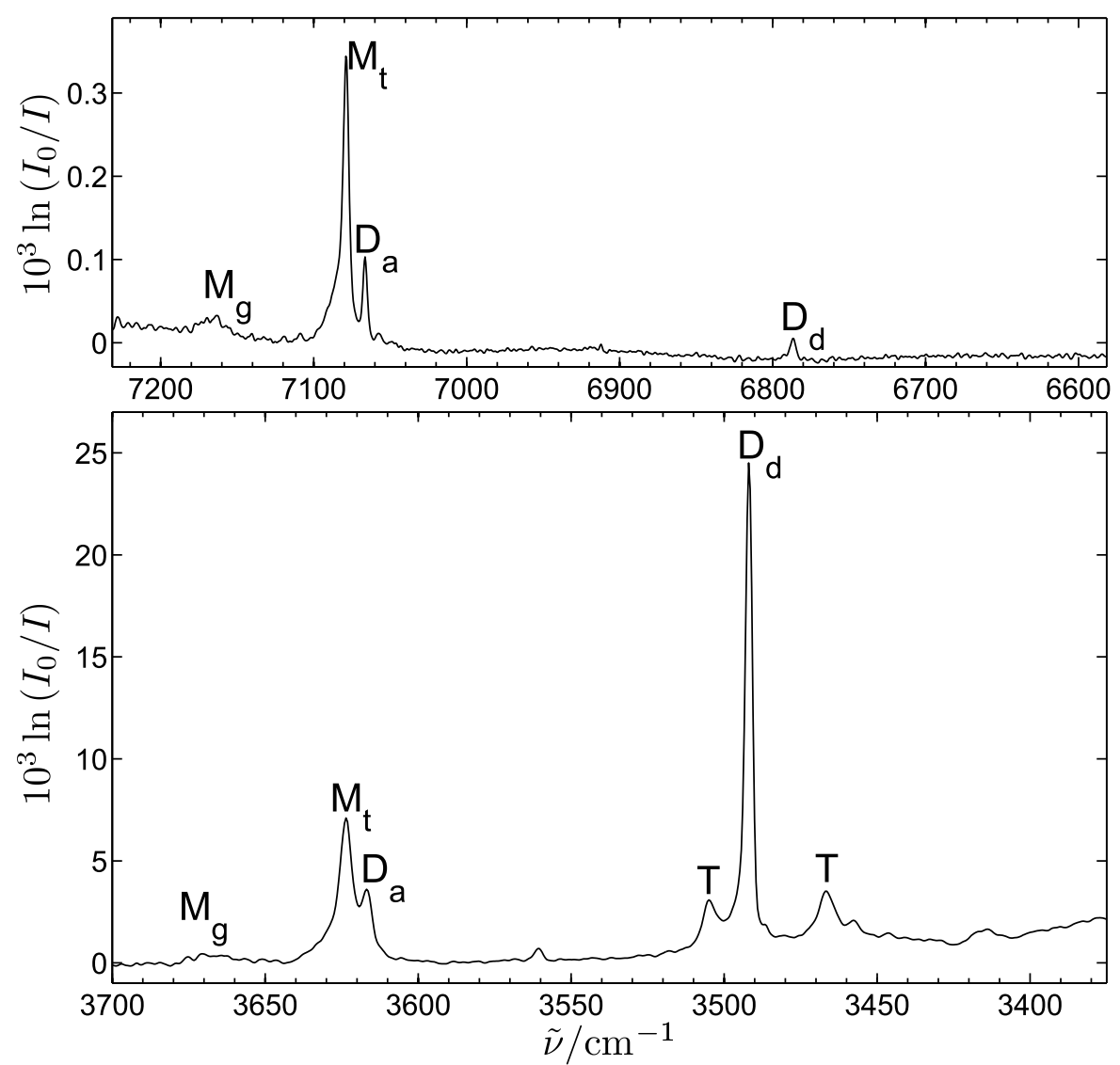

Abbildung 4.18.: Oberton (oben, 880 Pulse) und Fundamentalspektrum (unten, 100 Pulse) der Jetexpansionen von $0.6 \%$ Hexafluorisopropanol in 0.8 bar Helium im Bereich der $\mathrm{O}-\mathrm{H}$ Streckschwingung. Gekennzeichnet sind die Banden des transMonomer $\mathrm{M}_{\mathrm{t}}$, gauche-Monomer $\mathrm{M}_{\mathrm{g}}$, die Banden des Akzeptors $\mathrm{D}_{\mathrm{a}}$ und Donors $D_{d}$ des Dimers und der Trimere T. Bei dem Signal bei $3560 \mathrm{~cm}^{-1}$ könnte es sich um die Bande eines weniger stabilen Dimers handeln, etwa bestehend aus gauche-Donor und gauche-Akzeptor. Bei $6912 \mathrm{~cm}^{-1}$ ist sehr schwach ein Störsignal zu sehen, dass bei den Messungen verschiedener Substanzen auftrat.

sich als Trimersignale erklären. ${ }^{47} \mathrm{Im}$ Vergleich zu den Trimersignalen anderer Alkohole weisen sie eine geringe Intensität und Verschiebung zum Dimer auf, was sich mit einer offenen Struktur des Hexafluorisopropanoltrimers erklären lässt. ${ }^{47}$ Diese sollte weniger Kooperativität aufzeigen als die sonst häufig geschlossenen Ringstrukturen anderer Alkoholtrimere, sodass die Intensitätseffekte und Wellenzahlverschiebungen durch die Wasserstoffbrückenbindungen vergleichsweise gering ausfallen. Im Obertonspektrum können keine Trimersignale gefunden werden, daher müssen die Trimerobertöne und -kombinationen mindestens um einen Faktor 1000 gegenüber dem intensivsten Trimerübergang abgeschwächt sein.

In Zukunft könnten die hier präsentierten Jetexperimente noch durch Gasphasenmessungen ergänzt werden, mit denen einerseits durch verbesserte Auflösung der eventuellen Tunneldyna- 
mik des gauche-Monomers von Hexafluorisopropanol nachgespürt werden könnte. Andererseits könnten mit Hilfe druckverbreiterter Spektren von Hexafluorisopropanol in der Gasphase die Intensitätsverhältnisse und die absolute Intensität der $\mathrm{O}-\mathrm{H}$ Streckschwingungsfundamentale ${ }^{184}$ um die Werte der Obertöne ergänzt werden. So könnten die Intensitätseffekte im Dimer mit dem Monomer verglichen werden.

\subsubsection{Diskussion}

Nachdem in den vorangehenden Abschnitten die Ergebnisse aus Obertonexperimenten von verschiedenen Alkoholen ausgewertet wurden, sollen hier die Ergebnisse untereinander und mit quantenchemischen Rechnungen verglichen werden. In Tabelle 4.10 sind die wesentlichen Resultate für die $\mathrm{O}-\mathrm{H}$ Streckschwingungen der Donoren von Alkoholdimeren aus dieser Arbeit zusammengefasst und durch die Daten zu Trifluorethanol ${ }^{43}$ ergänzt. Da die $\mathrm{O}-\mathrm{H}$ Oszillatoren in den Molekülen nicht vollständig isoliert sind, ist es nicht möglich sie als Morseoszillatoren zu behandeln und ohne weiteres zusätzliche Parameter aus den spektroskopischen Messungen abzuleiten, wie etwa der harmonischen Wellenzahl oder der Dissoziationsenergie und Längenänderung der $\mathrm{O}-\mathrm{H}$ Bindung.

Tabelle 4.10.: Vergleich der Effekte von Wasserstoffbrücken auf die O-H Streckschwingungen der Donoren in Alkoholdimeren: Verschiebung der Dimerdonor- zur Monomerfundamentale $\Delta \tilde{\nu}_{\mathrm{OH}}^{\mathrm{D}_{\mathrm{d}}-\mathrm{M}}$ und diagonale Anharmonizität des Dimerdonors $x_{\mathrm{OH}, \mathrm{OH}}^{\mathrm{D}_{\mathrm{d}}}$ (beide in $\mathrm{cm}^{-1}$ ), sowie die relative Erhöhung der Anharmonizität des Dimerdonors gegenüber dem Monomer als Faktor $\frac{x_{\mathrm{OH}, \mathrm{OH}}^{\mathrm{D}_{\mathrm{H}}}}{x_{\mathrm{OH}, \mathrm{OH}}^{\mathrm{M}}}$, das Fundamentale-zu-Oberton Verhältnis der Dimerdonorschwingung $\frac{S_{01}^{\mathrm{D}_{\mathrm{d}}}}{S_{02}^{\mathrm{D}_{\mathrm{d}}}}=\frac{\int_{\nu_{01}} \ln \left(\frac{I_{0}}{I_{\mathrm{D}_{\mathrm{d}}}}\right) \mathrm{d} \tilde{\nu}}{\int_{\nu_{02}} \ln \left(\frac{I_{0}}{I_{\mathrm{D}_{\mathrm{d}}}}\right) \mathrm{d} \tilde{\nu}}$ und die relative Erhöhung gegenüber dem Verhältnis des Monomers als Faktor $\frac{S_{01}^{\mathrm{D}_{\mathrm{d}}} S_{02}^{\mathrm{M}}}{S_{02}^{\mathrm{D}_{2}} S_{01}^{\mathrm{M}}}$. Dazu wurden für die aliphatischen Alkohole die Monomerintensitätsverhältnisse aus der Gasphase ${ }^{116}$ verwendet, während für die fluorierten Alkohole die Verhältnisse aus Jetexpansionen genutzt wurden. Die Werte für Ethanol beziehen sich auf den Donor des stabilsten Dimers $D_{d}^{g g}$.

\begin{tabular}{llllcc}
\hline & $\Delta \tilde{\nu}_{\mathrm{OH}}^{\mathrm{D}_{\mathrm{d}}}-\mathrm{M}$ & $x_{\mathrm{OH}, \mathrm{OH}}^{\mathrm{D}_{\mathrm{d}}}$ & $\frac{x_{\mathrm{OH}, \mathrm{OH}}^{\mathrm{D}_{\mathrm{d}}}}{x_{\mathrm{OH}, \mathrm{OH}}^{\mathrm{M}}}$ & $\frac{S_{01}^{\mathrm{D}_{\mathrm{d}}}}{S_{02}^{\mathrm{D}_{\mathrm{d}}}}$ & $\frac{S_{01}^{\mathrm{D}_{\mathrm{d}}} S_{02}^{\mathrm{M}}}{S_{02}^{\mathrm{D}_{\mathrm{d}}} S_{01}^{\mathrm{M}}}$ \\
\hline Methanol & $-110(1)$ & $-99.2(4)$ & $1.156(9)$ & $320(80)$ & $26(7)$ \\
Ethanol & $-127.8(4)$ & $-101.1(4)$ & $1.150(8)$ & $400(100)$ & $50(10)$ \\
tert -Butylalkohol $^{\text {M }}$ & $-145.2(4)$ & $-102.6(4)$ & $1.179(5)$ & $1000(400)$ & $150(60)$ \\
Trifluorethanol $^{43}$ & -103 & -98 & 1.15 & $400(100)$ & $\leq 31(9)$ \\
Hexafluorisopropanol & $-131.7(5)$ & -98.6 & $1.171(7)$ & $600(200)$ & $\leq 40(20)$ \\
\hline
\end{tabular}

Die deutlich größeren Rotverschiebungen der Donor- zur Monomerfundamentalen bei zunehmender Anzahl von Methylsubstituenten können als robuster Hinweis darauf gewertet werden, 


\section{4. $O-H$ Streckschwingungen}

dass die Stärke der Wasserstoffbrückenbindung vom Methanol- über das Ethanol- zum tertButylalkoholdimer zunimmt. Dies kann vor allem auf die verbesserten Akzeptoreigenschaften der höher substitierten aliphatischen Alkohole zurück geführt werden. ${ }^{41}$ Der Effekt der Wasserstoffbrückenstärke ist für die Anharmonizitätskonstanten weitaus subtiler, da die Differenzen hier eine Größenordnung geringer ausfallen. Der Vergleich der absoluten Werte täuscht eine stetige Zunahme der diagonalen Anharmonizität mit stärkerer Wasserstoffbrücke, beziehungsweise höherer Rotverschiebung vor. Wird die relative Zunahme der Anharmonizität des Donors gegenüber dem jeweiligen Monomer betrachtet, so fällt diese im Ethanoldimer tendenziell geringer aus als im Methanoldimer, jedoch lässt die Größe der involvierten Fehlergrenzen hier eine experimentelle Auflösung dieser Werte kaum zu. Für den Dimerdonor im tert-Butylalkoholdimer kann jedoch klar die größte Zunahme der Anharmonizität gegenüber dem Monomer verzeichnet werden. Am deutlichsten wirkt sich die Wasserstoffbrückenstärke aber auf die Intensitätsverhältnisse von Fundamentale zu Oberton aus. Diese Verhältnisse steigen mit zunehmender Stärke der Wasserstoffbrückenbindung und unterscheiden sich, wenn man die relative Änderungen der Donorverhältnisse zu den entsprechenden Monomeren betrachtet, um Vielfache voneinander.

Im Falle der fluorierten Alkohole ändern sich die Effekte etwas. Zunächst liegen zwar in den Monomeren von Trifluorethanol ${ }^{188}$ und Hexafluorisopropanol ${ }^{184}$ bereits schwache intramolekulare $\mathrm{O}-\mathrm{H} \cdots \mathrm{F}$ Wasserstoffbrücken vor, aber ob sie sich auch signifikant auf spektroskopische Größen auswirken ist fraglich, da der experimentelle Vergleich $\mathrm{zu}$ den ungebundenen $\mathrm{O}-\mathrm{H}$ Gruppen fehlt. Im Vergleich zu den aliphatischen Alkoholen zeigen die fluorierten Alkohole höhere absolute Monomerintensitäten. Dies muss aber nicht in den intramolekularen Wasserstoffbrücken begründet sein, da zumindest harmonische Rechnungen der energetisch ungünstigeren Monomerkonformation von Trifluorethanol ohne Wasserstoffbrücke sogar eine höhere Intensität vorhersagen. ${ }^{47}$ Auch scheinen die Fundamentale-zu-Oberton Intensitätsverhältnisse etwas höher zu sein als bei den aliphatischen Alkoholen. Beide Beobachtungen ließen sich aber auch auf den Einfluss der Trifluormethyl-Substituenten auf die $\mathrm{O}-\mathrm{H}$ Bindung zurückführen. Die Bestimmung der Übergangsintensitäten ist bei den fluorierten Alkoholen problematisch. Während im Filet-Jet auf Grund der beschriebenen Sättigungseffekte zu kleine Fundamentale-zu-Oberton Verhältnisse bestimmt werden, können in der Gasphase bei Raumtemperatur Temperatureffekte eine Rolle spielen. Werden nämlich die intramolekularen Wasserstoffbrücken thermisch angeregt und damit geschwächt, so könnte das zu einer Verringerung der Fundamentalintensität und einer Erhöhung der Obertonintensität der O-H Streckschwingung führen - sofern die schwachen Wasserstoffbrücken einen Einfluss auf die Übergangsintensitäten haben. Somit könnte auch der Gasphasenwert für Trifluorethanol ${ }^{116}$ von 15(1) zu gering für ihr Verhältnis zueinander sein.

Die höhere Verschiebung der Donorfundamentale des Hexafluorisopropanoldimers zum Monomerübergang deutet auf eine stärkere Wasserstoffbrücke als im Trifluorethanoldimer hin. Auch nimmt im Falle von Hexafluorisopropanol die diagonale Anharmonizität vom Monomer zum Dimerdonor signifikant stärker zu. Die Unterschiede in Rotverschiebung und Anharmonizitätszunahme zwischen Trifluorethanol und Hexafluorisopropanol sind vergleichbar mit den Unterschieden zwischen Methanol und tert-Butylalkohol. Jedoch fällt bei den fluorierten Alkoholen der Unterschied der Intensitätsverhältnisse von Fundamentale zu Oberton weit weniger ausgeprägt aus. Während sich der Effekt der Dimerwasserstoffbrücke auf das Fundamentale-zuOberton Verhältnis von Methanol zu tert-Butylalkohol etwa versechsfacht, ist auf Grund des 
geringen Effekts vom Trifluorethanoldimer zum Hexafluorisopropanoldimer ein Unterschied innerhalb der experimentellen Fehlergrenzen nicht quantifizierbar.

Es konnte an den Beispielen der Dimere von Ethanol und besonders tert-Butylalkohol, Trifluorethanol ${ }^{43}$ und Hexafluorisopropanol ${ }^{47}$ gezeigt werden, dass die anharmonischen Effekte der Wasserstoffbrücke auf die $\mathrm{O}-\mathrm{H}$ Streckschwingung der Akzeptoren gering ausfallen. So wird im Allgemeinen die diagonale Anharmonizität der Akzeptoren in aliphatischen Alkoholdimeren um etwa $-1 \mathrm{~cm}^{-1}$ erhöht, während sie beim Dimer von Trifluorethanol nahezu unverändert bleibt ${ }^{43}$ und im Dimer von Hexafluorisopropanol um $1 \mathrm{~cm}^{-1}$ sinkt. Die Fundamentale-zu-Oberton Verhältnisse der Akzeptoren verdoppeln sich etwa gegenüber den Monomeroszillatoren.

Da in der Vergangenheit die Obertonspektren der Alkoholdimere nicht zugänglich waren, wurden die Anharmonizitätskonstanten aus den Wellenzahlen der $\mathrm{O}-\mathrm{H}$ Fundamentalübergänge der Alkoholsysteme und den O-D Fundamentalen der O-deuterierten Isotopologen abgeschätzt. ${ }^{36,59,188}$ Dies geschieht unter der Annahme von lokalisierten Morse-Oszillatoren, sodass sich sowohl für $\mathrm{O}-\mathrm{H}$ und $\mathrm{O}-\mathrm{D}$ die gleiche Kraftkonstante der Bindung ergibt und jeglicher Unterschied in den Schwingungsfrequenzen auf die unterschiedlichen reduzierten Massen zurückgeführt werden kann. Daraus ergeben sich die folgende Zusammenhänge ${ }^{36,59,188}$ für die harmonische Wellenzahl $\omega_{\mathrm{e}}$ und die Anharmonizitätskonstante $\omega_{\mathrm{e}} x_{\mathrm{e}}$ aus den experimentellen Wellenzahlen der $\mathrm{O}-\mathrm{H}$ Streckschwingung $\tilde{\nu}_{\mathrm{OH}}$ und der $\mathrm{O}-\mathrm{D}$ Streckschwingung $\tilde{\nu}_{\mathrm{OD}}$, sowie dem Verhältnis ihrer reduzierten Massen $r=1.8868$ :

$$
\begin{gathered}
\omega_{\mathrm{e}}=\frac{\tilde{\nu}_{\mathrm{OD}} \cdot r-\tilde{\nu}_{\mathrm{OH}}}{\sqrt{r}-1} \\
\omega_{\mathrm{e}} x_{\mathrm{e}}=\frac{\omega_{\mathrm{e}}-\tilde{\nu}_{\mathrm{OH}}}{2}
\end{gathered}
$$

In Tabelle 4.11 werden die Ergebnisse aus dieser Analyse der O-D und O-H Streckschwingungen („OD Analyse“) mit den experimentellen Werten durch die Obertonmessungen verglichen. Es zeigt sich grundsätzlich eine schlechte Übereinstimmung zwischen den Werten. Da die Anharmonizitätskonstanten die aus den Obertönen berechnet wurden als viel verlässlicher angenommen werden können, kann somit auf erhebliche Defizite der OD Analyse geschlossen werden. Sie überschätzt die Anharmonizitäten der Monomere und Dimerakzeptoren und unterschätzt die Anharmonizitätskonstanten der Dimerdonoren. Einzig der Wert des Hexafluorisopropanoldonors liegt bei Analyse der O-D Streckschwingungsspektren höher als die Anharmonizitätskonstante auf Grundlage des Obertons. Dies könnte aber angesichts der systematischen Abweichung der anderen Donorwerte eher auf eine Fehlinterpretation der Dimerdonor O-D Fundamentale als Dublett durch Fermiresonanz zurückzuführen sein (siehe Abb. A.2 im Anhang). Folglich wird zwar die Erhöhung der Anharmonizität vom Monomer zum Dimerdonor qualitativ in den Systemen richtig vorhergesagt (mit Ausnahme von Trifluorethanol), aber sie fällt in der O-D-Analyse zu gering aus. Die subtilen Unterschiede zwischen den freien Oszillatoren der Monomere und Dimerakzeptoren werden im Falle der fluorierten Alkohole durch die OD Analyse auch qualitativ nicht erfasst.

Die in dieser Arbeit gewonnenen Ergebnisse über die anharmonischen Effekte durch Wasserstoffbrücken auf die $\mathrm{O}-\mathrm{H}$ Streckschwingung in Alkoholdimeren sind besonders geeignet um 


\section{4. $\mathrm{O}-\mathrm{H}$ Streckschwingungen}

Tabelle 4.11.: Vergleich der Anharmonizitätskonstanten, die durch $\mathrm{O}-\mathrm{H}$ Obertonspektroskopie gewonnen wurden, mit den Werten aus der Analyse von O-D Spektren der O-deuterierten Systeme: Anharmonizitätskonstanten der O-H Streckschwingung der Monomere $x_{\mathrm{OH}, \mathrm{OH}}^{\mathrm{M}}$, Dimerakzeptoren $x_{\mathrm{OH}, \mathrm{OH}}^{\mathrm{Da}}$ und Dimerdonoren für Methanol, Trifluorethanol und Ethanol (gauche Monomer $\mathrm{M}_{\mathrm{g}}$, stabilstes Dimer D ${ }^{\mathrm{gg}}$ ) wurden bereits publiziert. Die Spektren des O-deuterierten tert-Butylalkohols wurden von René Wugt Larsen gemessen und die erhaltenen Bandenpositionen in der Dissertation von Julia Zischang veröffentlicht. ${ }^{67}$ O-deuteriertes Hexafluorisopropanol wurde von Tina Scharge im Zuge ihrer Promotion gemessen, ${ }^{43}$ die Ergebnisse zum trans-Monomer wurden bisher aber nicht veröffentlicht. Die Spektren und experimentellen Bandenzentren von tert-Butylalkohol-OD und HexafluorisopropanolOD sind im Anhang zu finden (Abbildung A.1 \& A.2); aus ihnen wurden die Anharmonizitäten unter Verwendung der Formeln 4.1 und 4.2 ermittelt. Da die Dimerdonorfundamentale der O-D Streckschwingung von Hexafluorisopropanol als Dublett erscheint, ist die daraus abgeleitete Anharmonizität unter Annahme einer Fermiresonanz berechnet (Vgl. Abb. A.2).

\begin{tabular}{|c|c|c|c|c|}
\hline & & $x_{\mathrm{OH}, \mathrm{OH}}^{\mathrm{M}}$ & $x_{\mathrm{OH}, \mathrm{OH}}^{\mathrm{D}_{\mathrm{a}}}$ & $x_{\mathrm{OH}, \mathrm{OH}}^{\mathrm{D}_{\mathrm{d}}}$ \\
\hline \multirow[t]{2}{*}{ Methanol } & Oberton & $-85.8(6)$ & & $-99.2(4)$ \\
\hline & OD-Analyse ${ }^{36}$ & $-87(4)$ & & $-89(4)$ \\
\hline \multirow[t]{2}{*}{ Ethanol } & Oberton & $-87.9(5)$ & $-88.2(6)$ & $-100.2(6)$ \\
\hline & OD-Analyse ${ }^{59}$ & $-90(4)$ & $-91(4)$ & $-93(4)$ \\
\hline \multirow[t]{2}{*}{ tert-Butylalkohol } & Oberton & $-87.0(2)$ & $-87.9(3)$ & $-102.6(4)$ \\
\hline & OD-Analyse & $-91(1)$ & $-91(2)$ & $-96(1)$ \\
\hline \multirow[t]{2}{*}{ Trifluorethanol } & Oberton $^{43}$ & -85 & -85 & -98 \\
\hline & OD-Analyse ${ }^{188}$ & -93 & -94 & -93 \\
\hline \multirow[t]{2}{*}{ Hexafluorisopropanol } & Oberton & $-84.2(4)$ & $-83.4(4)$ & $-98.6(4)$ \\
\hline & OD-Analyse & $-90(1)$ & $-93(2)$ & {$[-102(2)]$} \\
\hline
\end{tabular}

sie mit theoretischen Vorhersagen zu vergleichen, da die Jetspektroskopie die Systeme isoliert und bei tiefen Temperaturen untersucht. Für anharmonische Vorhersagen ist es besonders wichtig möglichst kleine Referenzsysteme zu haben, da die Rechenzeit nicht nur mit der Zahl der Elektronen des Systems steigt, sondern auch mit der Anzahl der Schwingungen. So konnten hier auf Grund der langen Rechenzeiten nur die Dimere von Methanol und Ethanol systematisch mit Hilfe quantenchemischer Methoden untersucht werden.

Die Rechnungen wurden mit Gaussian 09 Rev. D01 durchgeführt. ${ }^{68} \mathrm{Um}$ in anharmonischen Rechnungen imaginäre Frequenzen zu vermeiden, sind besonders enge Konvergenzkriterien für die Optimierungen nötig. So wurden MP2-Optimierungen mit dem ,Tight“-Kriterium durchgeführt. Auf B2PLYP-D3 Niveau wurde mit dem ,Tight“-Kriterium und „Ultrafine“-Gitter gearbeitet. Für B3LYP-D3 Rechnungen wurde das „VeryTight“-Kriterium sowie das „Superfine“-Gitter verwendet. Bei den anharmonischen Frequenzrechnungen wurde die Standardeinstellung von $0.025 \AA$ für die Schrittweite beibehalten. Die DFT-Funktionale wurden durch die empirischen D3-Dispersionskoeffizienten ${ }^{69}$ unter Verwendung der Becke-Johnson-Dämpfung ergänzt. ${ }^{70}$ 
In seiner aktuellen Version ist in Gaussian über die Störungstheorie zweiter Ordnung nicht nur die Berechnung anharmonischer Wellenzahlen implementiert, ${ }^{29}$ sondern auch die Vorhersage anharmonischer Intensitäten. ${ }^{21}$ Somit können hier neben den anharmonischen Wellenzahlen der Fundamentalübergänge auch die diagonalen Anharmonizitätskonstanten und Intensitätsverhältnisse von Fundamentale zu Oberton direkt zwischen Theorie und Experiment verglichen werden. In Tabelle 4.12 sind die entsprechenden Werte für Methanol und Ethanol zusammengetragen, wobei nur die Monomer- und Dimerdonorschwingungen betrachtet werden.

Es zeigen sich dabei systematische Unterschiede in der Qualität der Ergebnisse zwischen den verschiedenen Rechenmethoden. Ein systematischer Zusammenhang zwischen Abweichungen von den experimentellen Werten und der Größe der verwendeten Basissätze (cc-pVTZ > 6$311+\mathrm{G}(2 \mathrm{~d}, \mathrm{p})>\mathrm{TZVP})$ zeigt sich jedoch nicht. MP2 überschätzt die absoluten Wellenzahlen der Monomere und unterschätzt die diagonale Anharmonizität des Monomeroszillators. Somit wird also von MP2 die freie $\mathrm{O}-\mathrm{H}$ Bindung in den Rechnungen als zu stark und zu steif beschrieben. Das Gegenteil ist der Fall für B2PLYP-D3 und B3LYP-D3: Hier werden zumindest die absoluten Wellenzahlen eher unterschätzt. Für das Methanolmonomer wird von beiden Rechenmethoden die diagonale Anharmonizität in guter Übereinstimmung oder mit minimal zu hohen Werten vorhergesagt. Das Ethanolmonomer zeigt in den DFT-Rechnungen aber etwas zu geringe Anharmonizitäten. So sind die O-H Bindungen bei diesen beiden Rechenmethoden zu schwach, ihre Anharmonizität wird aber recht gut beschrieben.

Auf allen Rechenniveaus wird die Rotverschiebung der Dimerdonorschwingung überschätzt, sodass auch bei fast allen die absolute Wellenzahl der Dimerdonorschwingung unterschätzt wird. Außerdem werden auf allen Rechenniveaus die Anharmonizitäten der Dimerdonoren überschätzt. B3LYP-D3 zeigt dabei die größte Abweichung bei Rotverschiebung und Dimerdonoranharmonizität. Daran zeigt sich, dass die hier verwendeten Rechenmethoden (und besonders B3LYP-D3) die mechanischen Effekte der Wasserstoffbrücke auf die $\mathrm{O}-\mathrm{H}$ Bindung des Dimerdonors klar zu hoch vorhersagen.

Die Änderungen der elektrischen Effekte vom Monomer zum Dimer werden ebenfalls auf allen Rechenniveaus überschätzt, zumindest in Bezug auf das Intensitätsverhältnis der Fundamentalübergänge zu den Obertönen. Denn diese anharmonischen Intensitätsverhältnisse werden für die Monomere auf fast allen Niveaus deutlich unterschätzt und für die Dimere bis auf wenige Ausnahmen überschätzt.

Für Alkoholmonomere ist es möglich anharmonische Frequenzvorhersagen auf höheren Rechenniveaus vorzunehmen. ${ }^{26,27,189}$ Diese Ansätze liegen für Dimere noch außer Reichweite, sodass die hier getesteten Rechenmethoden in ihrem Grad theoretischer Näherungen in etwa vorherigen anharmonischen Modellierungen zu Alkoholdimeren von Methanol, ${ }^{51,190-192}$ Ethanol ${ }^{181,193}$ und tert-Butylalkohol ${ }^{194}$ entsprechen. Die in diesem Abschnitt vorgestellten anharmonischen Vorhersagen sagen zwar qualitativ die Effekte der Wasserstoffbrücke richtig vorher, sind aber quantitativ nicht genau genug, um die experimentellen Ergebnisse innerhalb ihrer Fehlergrenzen zu reproduzieren. Sicherlich kann die dreifache Herausforderung, aus exakter Beschreibung der $\mathrm{O}-\mathrm{H}$ Bindung und der Wechselwirkung der Moleküle über die Wasserstoffbrücke, sowie einer akkuraten Schwingungsrechnung um die experimentellen Daten zu reproduzieren, von den hier verwendeten eher einfachen Rechenmethoden und dem störungstheoretischen Ansatz zur Schwingungsanalyse nicht bewältigt werden. Etwaige gute Übereinstimmungen der einzel- 
Tabelle 4.12.: Vergleich von Ergebnissen aus anharmonischen Schwingungsrechnungen mit experimentellen Resultaten zur O-H Streckschwingung in Methanol und Ethanol (gauche Monomer $\mathrm{M}_{\mathrm{g}}$, stabilstes Dimer $\mathrm{D}^{\mathrm{gg}}$ ): anharmonische Wellenzahlen der Fundamentalübergänge vom Monomer $\tilde{\nu}_{\mathrm{OH}}^{\mathrm{M}}$ und Dimerdonor $\tilde{\nu}_{\mathrm{OH}}^{\mathrm{D}_{\mathrm{d}}}$, diagonale Anharmonizitätskonstanten von Monomer $x_{\mathrm{OH}, \mathrm{OH}}^{\mathrm{M}}$ und Dimerdonor $x_{\mathrm{OH}, \mathrm{OH}}^{\mathrm{D}_{\mathrm{d}}}$, sowie anharmonische Intensitätsverhältnisse von Fundamentale zu Oberton des Monomers $\frac{S_{01}^{\mathrm{M}}}{S_{02}^{\mathrm{M}}}=\frac{\int_{\nu_{01}} \ln \left(\frac{I_{0}}{I_{\mathrm{M}}}\right) \mathrm{d} \tilde{\nu}}{\int_{\nu_{02}} \ln \left(\frac{I_{0}}{I_{\mathrm{M}}}\right) \mathrm{d} \tilde{\nu}}$ und Dimers $\frac{S_{01}^{\mathrm{D}_{\mathrm{d}}}}{S_{02}^{\mathrm{D}_{\mathrm{d}}}}$.

\begin{tabular}{lllllll}
\hline & $\tilde{\nu}_{\mathrm{OH}}^{\mathrm{M}}$ & $\tilde{\nu}_{\mathrm{OH}}^{\mathrm{D}_{\mathrm{d}}}$ & $x_{\mathrm{OH}, \mathrm{OH}}^{\mathrm{M}}$ & $x_{\mathrm{OH}, \mathrm{OH}}^{\mathrm{D}_{\mathrm{d}}}$ & $\frac{S_{01}^{\mathrm{M}}}{S_{02}^{\mathrm{M}}}$ & $\frac{S_{01}^{\mathrm{D}_{\mathrm{d}}}}{S_{02}^{\mathrm{D}_{\mathrm{d}}}}$ \\
\hline Methanol & $\mathbf{3 6 8 4 ( 1 )}$ & $\mathbf{3 5 7 4 . 5 ( 3 )}$ & $-\mathbf{8 5 . 8}(\mathbf{6})$ & $\mathbf{- 9 9 . 2 ( 4 )}$ & $\mathbf{1 2 . 1}(\mathbf{9})^{a}$ & $\mathbf{3 2 0}(\mathbf{8 0})$ \\
\hline Experiment & & & & & & \\
MP2/cc-pVTZ & 3706.0 & 3571.5 & -82.9 & -101.5 & 10.3 & 420 \\
MP2/6-311+G(2d,p) & 3685.9 & 3553.2 & -83.1 & -102.9 & 11.8 & 702 \\
MP2/TZVP & 3703.1 & 3577.5 & -84.5 & -101.8 & 7.9 & 536 \\
& & & & & & \\
B2PLYP-D3/cc-pVTZ & 3673.9 & 3550.2 & -86.1 & -102.9 & 6.4 & 362 \\
B2PLYP-D3/6-311+G(2d,p) & 3674.2 & 3542.4 & -86.1 & -103.3 & 7.6 & 623 \\
B2PLYP-D3/TZVP & 3665.5 & 3538.0 & -87.3 & -104.2 & 5.4 & 423 \\
& & & & & & \\
B3LYP-D3/cc-pVTZ & 3648.9 & 3500.7 & -86.6 & -108.1 & 5.2 & 329 \\
B3LYP-D3/6-311+G(2d,p) & 3660.6 & 3508.5 & -86.5 & -106.9 & 6.4 & 599 \\
B3LYP-D3/TZVP & 3638.5 & 3487.3 & -87.4 & -108.5 & 4.5 & 394
\end{tabular}

Ethanol

\begin{tabular}{llllccc}
\hline Experiment & $\mathbf{3 6 5 9 . 3}(\mathbf{4})$ & $\mathbf{3 5 3 1 . 2}(\mathbf{3})$ & $\mathbf{- 8 7 . 9}(\mathbf{5})$ & $\mathbf{- 1 0 1 . 1}(\mathbf{4})$ & $\mathbf{7 . 5 ( 4 )}$ & $\mathbf{4 0 0 ( 1 0 0 )}$ \\
MP2/6-311+G(2d,p) & 3659.2 & 3511.1 & -83.6 & -104.5 & 10.3 & 843 \\
MP2/TZVP & 3675.1 & 3533.8 & -84.9 & -103.4 & 7.1 & 784 \\
& & & & & & \\
B2PLYP-D3/6-311+G(2d,p) & 3660.9 & 3509.6 & -85.2 & -104.6 & 4.7 & 681 \\
B2PLYP-D3/TZVP & 3653.2 & 3489.5 & -85.8 & -104.1 & 4.6 & 607 \\
& & & & & & \\
B3LYP-D3/cc-pVTZ & 3630.3 & 3455.4 & -87.2 & -107.8 & 4.2 & 503 \\
B3LYP-D3/6-311+G(2d,p) & 3638.7 & 3476.9 & -87.2 & -109.1 & 5.0 & 656 \\
B3LYP-D3/TZVP & 3617.8 & 3452.0 & -87.9 & -108.6 & 3.6 & 558 \\
\hline $\bar{a}$ Casphas
\end{tabular}


nen Rechenmethoden sind wahrscheinlich eher Resultat von Fehlerkompensation als von guter Modellierung der realen Systeme. Die Rechnungen könnten jedoch noch verbessert und systematischer untersucht werden. So könnte die Korrektur des Basissatzüberlagerungsfehlers die Beschreibung der zwischenmolekularen Wechselwirkungen besonders im Falle von MP2 verbessern. Außerdem könnte eine systematischere Untersuchung der Basissatzeffekte zum Beispiel innerhalb der korrelationskonsistenten Dunningbasissätze eine ungefähre Abschätzung des Basissatzlimits der spektroskopischen Größen ermöglichen. So könnten die Fehlergrenzen der Rechenmethoden besser aufgezeigt werden und auf ihre grundsätzlichen Schwächen hingewiesen werden, sodass die theoretischen Ansätze möglicherweise verbessert werden können. Dies wäre nicht möglich ohne experimentelle Bezugswerte, wie die in diesem Kapitel beschriebenen anharmonischen Effekte durch Wasserstoffbrücken auf die O-H Streckschwingung in Alkoholdimeren. 



\section{O-H Torsionen}

Librationsschwingungen entstehen aus den Rotations- und Torsionsbewegungen von Molekülen, die durch die Ausbildung einer Wasserstoffbrücke bei der Clusterbildung gehemmt und so in zwischenmolekulare Moden umgewandelt werden. Auch die Ausbildung einer intramolekularen Wasserstoffbrücke kann innerhalb eines Moleküls die Torsion einer Hydridgruppe einschränken und in eine Libration umwandeln. Die Hinderung der Hydridtorsion führt zu einer Erhöhung der Schwingungswellenzahl und somit zu einer spektroskopischen Blauverschiebung. Librationsübergänge liefern Informationen über das intermolekulare Potential der Wasserstoffbrücke und die hohe Anharmonizität von Librationen stellt eine Herausforderung an quantenchemische Rechnungen dar. In der Gasphase wurden bisher nur wenige Moleküle auf die Librationsschwingungen ihrer Cluster untersucht.

Besonders umfangreich sind die Cluster von Fluorwasserstoff hinsichtlich ihrer Librationsschwingungen charakterisiert. Zu den Librationen des Dimerdonors lassen sich neben der aus der Ebene schwingenden Bewegung des verbrückten Wasserstoffatoms, ${ }^{195-197}$ auch Schwingungen innerhalb der Ebene der vier Atome zählen. Bei diesen Bewegungen nimmt auch das Wasserstoffatom des Dimerakzeptors signifikant teil. Ihre Übergänge wurden im Fundamental- ${ }^{198}$ und im Kombinationsbereich mit den H-F Streckschwingungen untersucht. ${ }^{197,199-201}$ Überdies wurden für das Trimer, ${ }^{202,203}$ Tetramer ${ }^{204}$ und Pentamer ${ }^{204}$ von Fluorwasserstoff, sowie für gemischte Dimere mit Oxiran ${ }^{205}$ und dem schwach gebundenen Komplex mit Kohlenmonoxid ${ }^{206}$ Librati- $^{2}$ onsschwingungen gemessen.

Für das nächsthöhere Homologe Chlorwasserstoff wurden Librationsübergänge des Dimers ${ }^{207,208}$ sowie der Komplexe mit Kohlenmonoxid ${ }^{206,209}$ und Cyanwasserstoff, ${ }^{210}$ bei denen Chlorwasserstoff als Wasserstoffbrückendonor fungiert, beobachtet. Für Cyanwasserstoff ist auch der Librationsübergang im gemischten Dimer mit Ammoniak bekannt. ${ }^{211}$ Für das Iodwasserstoffdimer und den gemischten Komplex mit Kohlenmonoxid sind die Übergänge der in der Ebene verlaufenden Librationsbewegungen bekannt. ${ }^{212}$

Von besonderem Interesse sind für diese Arbeit die Librationsschwingungen in $\mathrm{O}-\mathrm{H} \cdots \mathrm{O}$ Wasserstoffbrückensystemen, die neben Wasser ${ }^{38}$ vor allem für Alkoholcluster in der Gasphase beschrieben worden sind. Nach einer ersten Arbeit zu größeren Methanolclustern, ${ }^{213}$ wurden Librationsschwingungen des Tetramers, ${ }^{12}$ Trimers ${ }^{12,13}$ und Dimers ${ }^{14}$ charakterisiert. Auch Librationsübergänge von tert-Butylalkoholtrimer und -tetramer wurden veröffentlicht, ${ }^{13}$ ebenso die Raman-aktive Libration im Ameisensäuredimer. ${ }^{16}$ Die Librationen gemischter Dimere von Trifluorethanol wurden in der warmen Gasphase untersucht. ${ }^{214}$

In diesem Kapitel sollen die Torsionen freier O-H Gruppen und ihre Librationsschwingungen in Wasserstoffbrücken-gebundenen Clustern systematisch untersucht werden. Dazu werden die Ferninfrarotspektren von Methanol, tert-Butylalkohol, Ethanol, Trifluorethanol und Methyllaktat in Überschallexpansionen vorgestellt. Auch die IR-aktive Libration im Ameisensäuredimer 
wurde beobachtet und wird in Kapitel 6 beschrieben.

\subsection{Messbedingungen}

Die Torsions- und Librationsübergänge der O-H Gruppe können im Bereich von ungefähr 150 bis $1000 \mathrm{~cm}^{-1}$ beobachtet werden. Der größte Teil dieses Bereiches konnte durch Verwendung eines Bolometers (4 K, ,Bolometer_neu“) mit einem eingebauten Bandpassfilter $\left(200-690 \mathrm{~cm}^{-1}\right)$ abgedeckt werden. Um auch die Optiken des Filet-Jet auf diesen Spektralbereich anzupassen wurden CsI $\left(>200 \mathrm{~cm}^{-1}\right)$ Fenster und Linsen verwendet und ein $6 \mu$ Mylar Strahlteiler. Letzterer zeigt einen starken Einbruch seiner Transmission zwischen 370 und $390 \mathrm{~cm}^{-1}$, was an dieser Position zu starkem Rauschen und Artefakten in den Spektren führt. Deshalb ist dieser Bereich in den hier gezeigten Spektren nicht abgebildet. Auf Grund der langsamen Detektion des Bolometers wurde generell eine Spiegelgeschwindigkeit von $20 \mathrm{kHz}$ gewählt. Um die Gaspulse dennoch nicht zu lang werden zu lassen, wurde die Auflösung auf $4 \mathrm{~cm}^{-1}$ gesetzt. Als Lichtquelle diente der Globar des FTIR Spektrometers. Die Apertur wurde auf $4 \mathrm{~mm}$ gesetzt.

Für die komplementären Spektren im $\mathrm{O}-\mathrm{H}$ Streckschwingungsbereich wurde das InSbDetektorelement eines InSb/MCT Sandwichdetektors (77 K, „Sandwich_neu“) und Filter 14+7 $\left(2950-3900 \mathrm{~cm}^{-1}\right)$ verwendet. Das Spektrometer wurde mit einer Apertur von $4 \mathrm{~mm}, 80 \mathrm{kHz}$ Spiegelgeschwindigkeit, einer Auflösung von $2 \mathrm{~cm}^{-1}$, der Wolframlampe als Lichtquelle und einem $\mathrm{CaF}_{2}$ Strahlteiler betrieben. Als Optiken wurden $\mathrm{CaF}_{2}$ Fenster und Linsen verwendet.

Die Prozeduren mit denen die Substanzen und Helium in das Reservoir des Filet-Jet gefüllt wurden sind in Tabelle 5.1 zusammengefasst.

\subsection{Methanol}

Die OH-Torsion im isolierten Methanolmolekül erfolgt in einem Potential mit drei äquivalenten Minima, was zur Tunnelaufspaltung der Schwingungszustände in einen A-Zustand und zwei E-Zustände führt. Die Rotationszustände lassen sich näherungsweise mit zwei Quantenzahlen $K$ und $J$ beschreiben, wobei $K$ nur positive Werte für den A-Zustand und für die entarteten EZustände sowohl positive als auch negative Vorzeichen annehmen kann. Außerdem ergibt sich für den A-Zustand bei $K>0$ eine Feinaufspaltung, die die Rotationsniveaus in zwei Zustände teilt, die mit + und - gekennzeichnet werden können. ${ }^{175}$ Für $J$ gilt in allen Tunnelspezies $J \geq|K|$.

Für die Torsionsanregung ergeben sich verschiedene Auswahlregeln für Infrarotübergänge. So muss die Symmetrie der Torsionswellenfunktion erhalten bleiben: $\mathrm{A} \leftrightarrow \mathrm{A}$ und $\mathrm{E} \leftrightarrow \mathrm{E}$. Außerdem gelten $\Delta K= \pm 1$ und $\Delta J=0, \pm 1 .{ }^{175}$ Weitere Auswahlregeln sind für den Zweck dieser Arbeit nicht von Belang. ${ }^{175,215,216}$

\subsubsection{Methanol-OH}

Die Energie des ersten angeregten Zustands der Torsion von Methanol liegt nahe der Maxima ihres Potentials, sodass in $\mathrm{CH}_{3} \mathrm{OH}$ die Zustände mit $85.5 \mathrm{~cm}^{-1}$ um eine Größenordnung stärker 
Tabelle 5.1.: Experimentelle Bedingungen der im Zuge dieser Arbeit am Filet-Jet aufgenommenen Substanzspektren: Stagnationsdruck $p_{\text {st }}$, Sättigertemperatur $T_{\mathrm{S}}$ und Verdünnung auf die die Helium/Substanz Mischung des Sättigers mit Hilfe der Zumischung von Helium über eine weitere Zuleitung zum Reservoir gebracht wurde. Die Konzentrationen der Substanzen im Reservoir wurden aus ihren Gleichgewichtsdampfdrücken bei der Sättigertemperatur und einem Heliumdruck von 1.6 bar im Sättiger abgeschätzt.

\begin{tabular}{lcccc}
\hline & $p_{\text {st }} /$ bar & $T_{\mathrm{S}} /{ }^{\circ} \mathrm{C}$ & Verdünnung & Konzentration \\
\hline $\mathrm{CH}_{3} \mathrm{OH}$ & 0.4 & -10 & $100 \%$ & $1.3 \%$ \\
& 0.8 & -10 & $50 \%$ & $0.7 \%$ \\
$\mathrm{CD}_{3} \mathrm{OH}$ & 0.8 & -20 & $50 \%$ & $0.3 \%$ \\
& 0.8 & -10 & $50 \%$ & $0.7 \%$ \\
$\mathrm{CH}_{3} \mathrm{OD}$ & 0.8 & -20 & $50 \%$ & $0.3 \%$ \\
& 0.4 & -20 & $100 \%$ & $0.6 \%$ \\
$\mathrm{CD}_{3} \mathrm{OD}$ & 0.8 & -20 & $50 \%$ & $0.3 \%$ \\
& 0.8 & -10 & $50 \%$ & $0.7 \%$ \\
tert-Butylalkohol & 0.8 & -20 & $50 \%$ & $0.3 \%$ \\
& 0.8 & -10 & $50 \%$ & $0.7 \%$ \\
& 0.4 & 26 & $1.3 \%$ & $0.06 \%$ \\
tert-Butylalkohol-OD & 0.4 & 26 & $2.0 \%$ & $0.09 \%$ \\
& 0.6 & 26 & $2.0 \%$ & $0.09 \%$ \\
& 0.8 & 26 & $3.5 \%$ & $0.16 \%$ \\
Ethanol & 0.4 & 26 & $1.3 \%$ & $0.06 \%$ \\
Trifluorethanol & 0.4 & 26 & $2.0 \%$ & $0.09 \%$ \\
& 0.6 & 26 & $2.0 \%$ & $0.09 \%$ \\
& 0.8 & 26 & $3.5 \%$ & $0.16 \%$ \\
& 0.7 & 0 & $14 \%$ & $0.14 \%$ \\
Trifluorethanol-OD & 0.3 & -10 & $50 \%$ & $0.3 \%$ \\
rac.-Methyllaktat & 0.4 & -10 & $50 \%$ & $0.3 \%$ \\
S)-Methyllaktat & 0.6 & -15 & $50 \%$ & $0.2 \%$ \\
& 0.8 & -15 & $50 \%$ & $0.2 \%$ \\
& 0.9 & -15 & $50 \%$ & $0.2 \%$ \\
& 0.8 & -5 & $50 \%$ & $0.4 \%$ \\
& 0.9 & -5 & $50 \%$ & $0.4 \%$ \\
& 0.8 & -10 & $50 \%$ & $0.3 \%$ \\
& 0.7 & 15 & $100 \%$ & $0.1 \%$ \\
\hline
\end{tabular}




\section{5. $\mathrm{O}-\mathrm{H}$ Torsionen}

aufgespalten sind, als im Grundzustand mit $9.1 \mathrm{~cm}^{-1} .{ }^{175}$ Zusammen mit der starken Kopplung zwischen Torsion und $K$-Rotation führt die Tunnelaufspaltung zu einem komplexen Ferninfrarotspektrum von Methanol. Deshalb ist es hilfreich zunächst die Monomerübergänge von Methanol im Jet zuzuordnen, bevor die Cluster untersucht werden. In Referenz 175 sind ein Großteil der Schwingungs-Rotations-Linien von $\mathrm{CH}_{3} \mathrm{OH}$ im hier interessanten Intervall von 190 bis $680 \mathrm{~cm}^{-1}$ zusammengefasst. Da auch ihre Zuordnung und Intensität gegeben ist, kann somit aus den experimentellen Daten bei Raumtemperatur ein Spektrum bei niedrigerer Temperatur berechnet werden. Der integrierte Absorptionskoeffizient $S_{\mathrm{nm}}$ eines Übergangs von einem energetisch tieferen Zustand $\mathrm{m}$ in einen energetisch höheren Zustand $\mathrm{n}$ berechnet sich nach

$$
S_{\mathrm{nm}}=\frac{1}{4 \pi \epsilon_{0}} \frac{8 \pi^{3}}{3 c h} \tilde{\nu}_{\mathrm{nm}} \cdot \frac{g_{\mathrm{m}}}{Z} e^{-\frac{E_{\mathrm{m}}}{k T}}\left(1-e^{-\frac{h c \tilde{\nu}_{\mathrm{nm}}}{k T}}\right)\left\langle\mu_{\mathrm{nm}}\right\rangle^{2}
$$

aus der Wellenzahl des Übergangs $\tilde{\nu}_{\mathrm{nm}}$ und seinem Übergangsdipolmoment $\mu_{\mathrm{nm}}$, der Entartung $g_{\mathrm{m}}$ und Energie $E_{\mathrm{m}}$ von $\mathrm{m}$ und der Schwingungs-Rotations-Zustandssumme $Z$ des Moleküls. Sollen nun aus den gegebenen integrierten Absorptionskoeffizienten $S_{\mathrm{nm}}(\mathrm{GP})$ der Gasphase bei Raumtemperatur die entsprechenden Werte bei genäherten Temperaturen in Jetexpansionen $S_{\mathrm{nm}}$ (Jet) berechnet werden, so müssen lediglich die von der Temperatur abhängigen Terme ins Verhältnis gesetzt werden:

$$
\frac{S_{\mathrm{nm}}(\mathrm{GP})}{S_{\mathrm{nm}}(\mathrm{Jet})}=\frac{Z_{\mathrm{Jet}}}{Z_{\mathrm{GP}}} \frac{e^{-\frac{E_{\mathrm{m}}}{k T_{\mathrm{GP}}}}\left(1-e^{-\frac{h c \tilde{\nu}_{\mathrm{nm}}}{k T_{\mathrm{GP}}}}\right)}{e^{-\frac{E_{\mathrm{m}}}{k T_{\mathrm{Jet}}}}\left(1-e^{-\frac{h c \tilde{\nu}_{\mathrm{nm}}}{k T_{\mathrm{Jet}}}}\right)}
$$

Im thermischen Gleichgewicht gehören alle Zustände der gleichen Zustandssumme an, sodass bei Betrachtung relativer Intensitäten die Zustandssummen nicht relevant sind. In Überschallexpansionen liegen jedoch keine Gleichgewichtsbedingungen vor. So können sich für verschiedene Freiheitsgrade der Moleküle auch verschiedene effektive Temperaturen ergeben. Somit stellt Gleichung 5.2 nur eine der Näherung von Intensitäten in Jetexpansionen dar. In Abbildung 5.1 sind beispielhaft die $\mathrm{A} \leftrightarrow \mathrm{A}$ Übergänge dargestellt die für $\mathrm{CH}_{3} \mathrm{OH}$ bei $25 \mathrm{~K}$ im Intervall von 190 bis $680 \mathrm{~cm}^{-1}$ beobachtbar sein sollten. Diese sind als Pfeile zwischen den Niveaus mit verschiedener Rotationsquantenzahl $K$ und Schwingungsquantenzahl der Torsion $\tau$ dargestellt. Der Einfluss von $J$ auf die Energien der Zustände wird jedoch nicht beachtet, was für alle Niveaus

Abbildung 5.1.: Torsionsübergänge (in $\mathrm{cm}^{-1}$ ) von $\mathrm{CH}_{3} \mathrm{OH}$, die bei $25 \mathrm{~K}$ im spektroskopischen Fenster von $190-680 \mathrm{~cm}^{-1}$ für die A-Spezies erwartet werden. Die Niveaus sind im Energieschema mit der Schwingungsquantenzahl der Torsion $\tau$ und der Rotationsquantenzahl $K$ gekennzeichnet und ihre Energien $E(\tau, K)$ gegeben. Die Absorptionsspektren wurden aus dem Raumtemperaturspektrum ${ }^{175}$ für eine Auflösung von $4 \mathrm{~cm}^{-1}$ berechnet . Das obere Spektrum zeigt die Signale aller Methanolspezies, während das untere nur die $\mathrm{A} \leftrightarrow \mathrm{A}$ Übergänge zeigt. Übergänge, deren Intensität zu gering sind, sind mit gestrichelten Linien im Energieschema dargestellt. 


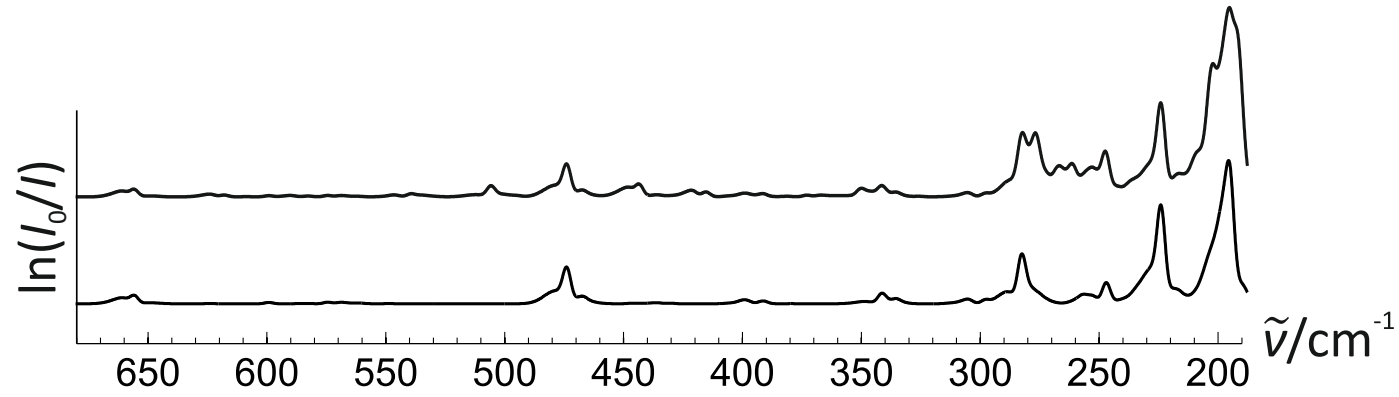

$(3,2)$

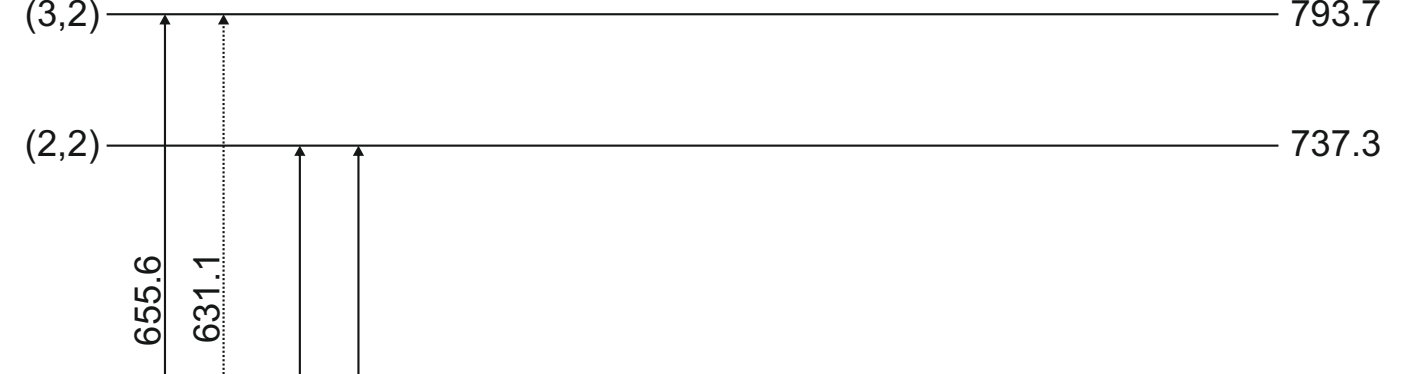

$(2,1)$

$(2,3)$

$(2,4)$

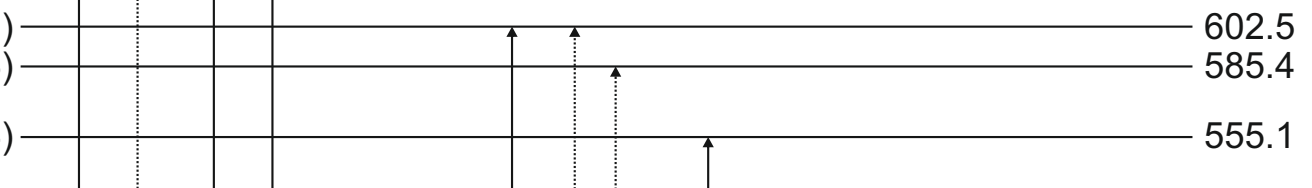

$(2,0)$

$(1,4)$

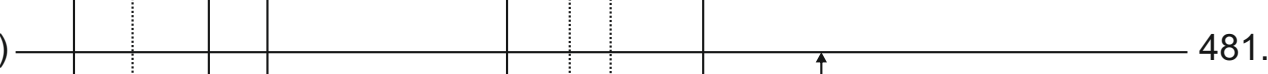

481.2

462.3

$(1,0)$
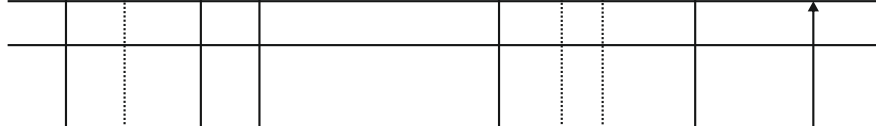

$(1,3)$

:

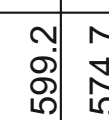

$(1,1)$
$(1,2)$

$(0,3)$

$(0,2)$

$(0,1)$

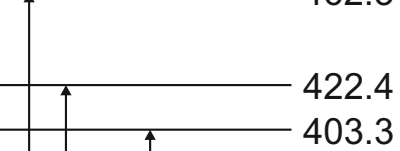

$(\tau, K)$

162.6

154.2

138.1

127.98

$E(\tau, K) / \mathrm{cm}^{-1}$ 


\section{5. $\mathrm{O}-\mathrm{H}$ Torsionen}

$J=0$ entsprechen würde. Dies ist für $K>0$ jedoch nur theoretisch möglich. Zusätzlich sind Absorptionsspektren für $25 \mathrm{~K}$ gezeigt, die aus Umrechnung der Intensitäten aus den Raumtemperaturdaten ${ }^{175}$ erhalten und durch Darstellung der Linien als Gausskurven mit $4 \mathrm{~cm}^{-1}$ Halbwertsbreite aufgetragen wurden. Neben dem vollständigen Spektrum bei $25 \mathrm{~K}$ ist auch ein Spektrum gezeigt, dass nur die $\mathrm{A} \leftrightarrow \mathrm{A}$ Übergänge enthält. Aus der Abbildung lässt sich erkennen, dass in angeregten Torsionszuständen die Verteilung der $K$-Quantenzahlen keiner energetischen Sortierung folgt und so auch die Wellenzahlen der Übergänge im Spektrum keiner einfachen Abfolge entsprechen. Jedoch steigt die Energie der einzelnen $K$-Zustände mit steigendem $J$ monoton, sodass sich für jeden Übergang zwischen Zuständen mit unterschiedlichem $K$ Rotationszweige entsprechend den möglichen Änderungen von $J$ ergeben. Das Spektrum bei $25 \mathrm{~K}$ ist jedoch von den Q-Zweigen dominiert, da die P- und R-Zweige auf Grund der relativ großen Rotationskonstante von $\mathrm{CH}_{3} \mathrm{OH}$ (diese lässt sich für niedrige $J$ mit $\frac{\tilde{B}+\tilde{C}}{2} \approx 0.8 \mathrm{~cm}^{-1}$ nähern) breit auslaufen. Die $K$-Niveaus des Torsionsgrundzustands sind nur bis bis $K=3$ ausreichend besetzt um relevante Fundamentalintensitäten zu besitzen. Dies liegt auch daran, dass die niedrigsten Niveaus der Zustände $K>0$ tatsächlich höher liegen als im Energiediagramm dargestellt, da, wie weiter oben erwähnt, zum Beispiel $J=3$ das niedrigste Niveau in $K=3$ ist, also etwa $12 \cdot 0.8 \mathrm{~cm}^{-1}$ höher liegt. Die ersten Obertonübergänge der Torsion besitzen eine um etwa den Faktor 5 geringere Intensität, sodass hier Übergänge aus höheren Niveaus $(K>1)$ kaum noch relevante Intensität besitzen. Daher ist der Obertonbereich bei $25 \mathrm{~K}$ deutlich leichter zu analysieren als der Fundamentalbereich, in dem viele Banden überlappen.

Für die Übergänge zwischen den E-Zuständen lassen sich ähnliche Schlüsse ziehen: Für die Fundamentalübergänge lassen sich mehr Banden erwarten, da hier die Intensitäten der Übergänge aus höher angeregten Zuständen $(K= \pm 3)$ noch hoch genug sind, während für die Obertonübergänge fast nur die Übergänge aus energetisch niedrigsten K-Zuständen ausreichende Intensität aufweisen (energetische Abfolge im Torsionsgrundzustand: $K=-1,0,1,-2,2$ ).

Diese berechneten Spektren lassen sich nutzen, um die Übergänge der Methanolmonomere in Jetexpansionen zu identifizieren und ihnen eine ungefähre Temperatur zuzuordnen. Die Kühlung von $7 \% \mathrm{CH}_{3} \mathrm{OH}$ in 1.9 bar Argon in einer Jetexpansion durch eine $0.1 \mathrm{~mm}$ Lochdüse wurde bereits anhand der $\mathrm{C}-\mathrm{O}$ Streckschwingung untersucht. ${ }^{217}$ Dabei wurden keine heißen Torsionsbanden beobachtet, die Torsionsschwingung wurde also effektiv gekühlt. Außerdem wurde ermittelt, dass sich das Besetzungsverhältnis der E- und A-Spezies zueinander in der Expansion im Vergleich zum Raumtemperaturverhältnis nicht ändert, ferner wurden sogar unterschiedliche Temperaturen den A- und E-Spezies zugeordnet. Dies spricht dafür, dass es während einer Jetexpansion nicht zu einem Wechsel der Tunnelsymmetrie der Moleküle kommt.

Um die Annahme einer Erhaltung der Tunnelsymmetrie in die Berechnung der Monomerspektren aus den Raumtemperaturdaten einfließen zu lassen, muss zum einen für die berechneten Jetspektren die um $5.5 \mathrm{~cm}^{-1}$ höhere Nullpunktsenergie der E-Spezies beachtet werden (entspricht dem Energieunterschied des energetisch tiefsten E-Niveaus mit $K=-1$ und $J=1$ und dem niedrigsten A-Niveau mit $K=0$ und $J=0) .{ }^{175}$ Zum anderen müssen auch die Zustandssummen der Spezies getrennt voneinander betrachtet werden, da sie sich relativ zu den Raumtemperatursummen bei Abkühlung auf tiefe Temperaturen unterschiedlich stark ändern können. Da die Energieniveaus von $\mathrm{CH}_{3} \mathrm{OH}$ in Referenz 175 bis etwa $1000 \mathrm{~cm}^{-1}$ gegeben sind, können die 
kombinierten Zustandssummen der Rotation und Schwingung über

$$
Z_{\text {vibrot }}=\sum g_{\mathrm{i}} \cdot e^{-\frac{E_{i}}{k T}}
$$

durch Summation über den Großteil der energetisch und statistisch relevanten Rotationsschwingungszustände berechnet werden. Für die Zustandssummen der Translation muss davon ausgegangen werden, dass sie sich durch Überschallexpansionen nicht unterschiedlich für die E- und A-Spezies ändern. Die elektronische Zustandssumme kann bei Raumtemperatur und in Jetexpansionen vernachlässigt werden. In Tabelle 5.2 sind die Verhältnisse beider Zustandssummen bei verschiedenen Temperaturen $Z_{T}$ zur jeweiligen Raumtemperaturzustandssumme $Z_{\mathrm{RT}}$ gegeben, die in Gleichung 5.2 eingesetzt werden können um die entsprechenden Intensitäten der Tunnelspezies zu berechnen.

Tabelle 5.2.: Verhältnisse der Zustandssummen bei tiefen Temperaturen $Z_{T}$ der A- und ESpezies von $\mathrm{CH}_{3} \mathrm{OH}$ zu den jeweiligen Zustandssummen bei Raumtemperatur $Z_{\mathrm{RT}}$. Die absoluten Werte der Zustandssumme sind in Tabelle A.1 im Anhang gegeben.

\begin{tabular}{lll}
\hline & $\frac{Z_{T}(A)}{Z_{\mathrm{RT}}(A)}$ & $\frac{Z_{T}(E)}{Z_{\mathrm{RT}}(E)}$ \\
\hline $50 \mathrm{~K}$ & 0.041 & 0.049 \\
$35 \mathrm{~K}$ & 0.023 & 0.028 \\
$25 \mathrm{~K}$ & 0.013 & 0.017 \\
$15 \mathrm{~K}$ & 0.005 & 0.008 \\
\hline
\end{tabular}

Dabei ergeben sich für beide Tunnelspezies sehr ähnliche, aber für die E-Spezies etwas größere Verhältnisse. Das heißt, dass für die A-Spezies bei Abkühlung die Anzahl der besetzten Zustände stärker abnimmt als für die E-Spezies. Dies führt nach Gleichung 5.2 wiederum zu höheren Intensitäten der $\mathrm{A} \leftrightarrow \mathrm{A}$ Übergänge gegenüber den $\mathrm{E} \leftrightarrow \mathrm{E}$ Übergängen aus dieser statistischen Gewichtung. Diese Gewichtung wirkt dem Effekt der um $5.5 \mathrm{~cm}^{-1}$ höheren Nullpunktsenergie der E Zustände entgegen, sodass insgesamt kein signifikanter Intensitätseffekt in dem Temperaturintervall von 15 bis $50 \mathrm{~K}$ allein durch die Konservierung der Tunnelsymmetrien erwartet werden kann. Deshalb wird in den hier berechneten Spektren der Einfachheit halber ein Gleichgewicht zwischen der E- und A-Spezies angenommen. Diese Näherung betrifft nur die relativen Intensitäten der $\mathrm{E} \leftrightarrow \mathrm{E}$ gegenüber den $\mathrm{A} \leftrightarrow \mathrm{A}$ Übergängen und erscheint gering gegenüber den anderen Näherungen dieser Betrachtung wie der Annahme vergleichbarer effektiver Temperaturen für die Torsion und $K$-Rotation. Der in Referenz 217 beobachtete Effekt ist somit eher auf die unterschiedlichen Temperaturen der E- und A-Spezies zurückzuführen.

In Abbildung 5.2 werden experimentelle Jetspektren mit berechneten Spektren im Ferninfrarotbereich verglichen. Die Jetspektren wurden bei unterschiedlichen Stagnationsdrücken gemessen: Spektrum b zeigt Expansionen von $1.3 \% \mathrm{CH}_{3} \mathrm{OH}$ in 0.4 bar Helium und Spektrum c $0.7 \%$ $\mathrm{CH}_{3} \mathrm{OH}$ in 0.8 bar Helium. Die berechneten Spektren für Temperaturen von $50 \mathrm{~K}$ (a) und $25 \mathrm{~K}$ (d) wurden so skaliert, dass die Intensität der $\mathrm{A} \rightarrow \mathrm{A}$ Obertonbande $(\tau=0 \rightarrow 2, K=0 \rightarrow 1)$ bei $475 \mathrm{~cm}^{-1}$ mit den gemessenen Jetspektren übereinstimmt. Dabei ist eine gute Übereinstimmung zwischen den Jetspektren (b,c) und den berechneten Spektren (a,d) festzustellen. Durch den Vergleich lassen sich auch leicht die intensiven Methanolclusterbanden in den Jetspektren 


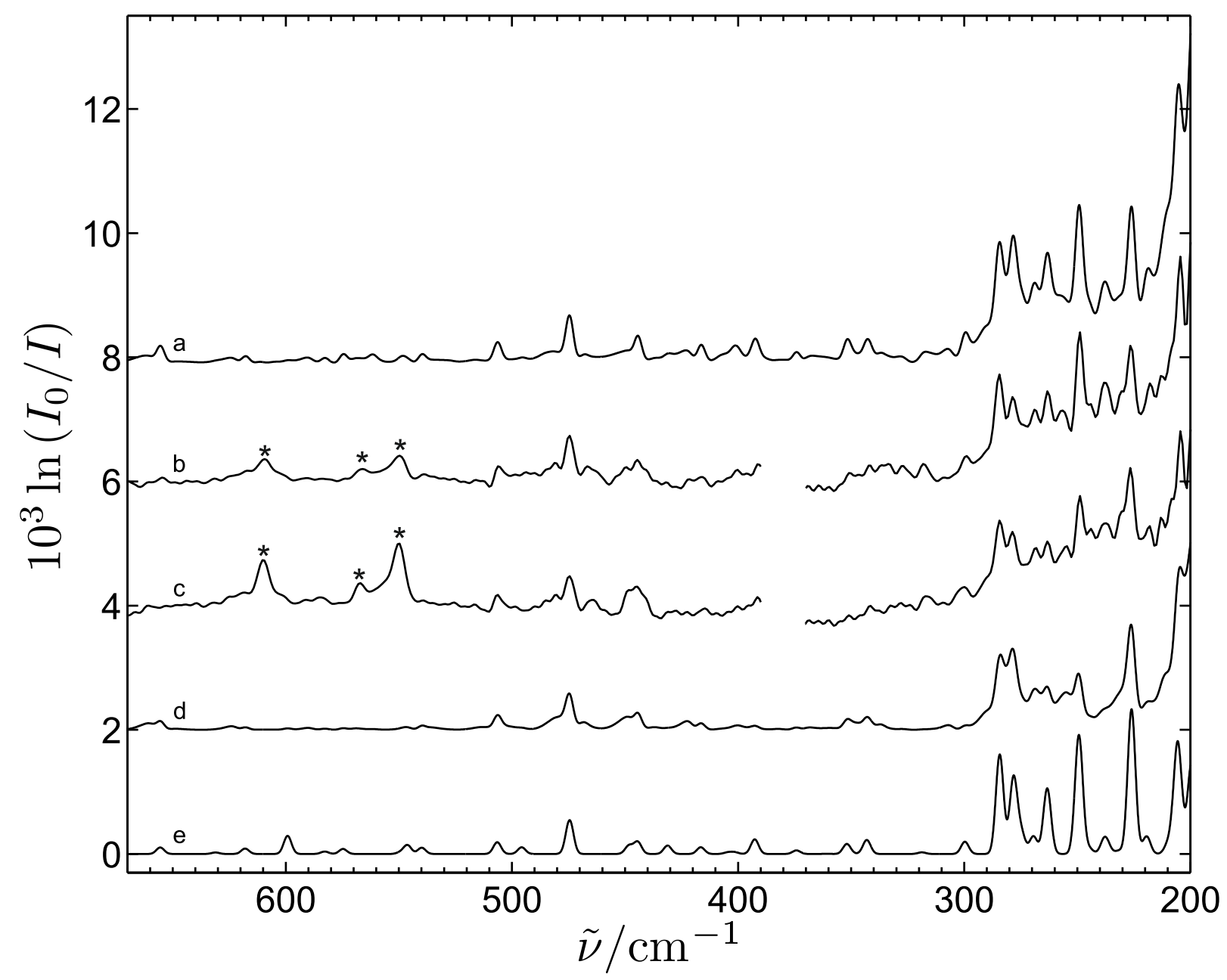

Abbildung 5.2.: Methanolspektren im Ferninfrarotbereich: (a) berechnetes MethanolMonomerspektrum für $50 \mathrm{~K}$, (b) Jetexpansionen von $1.3 \% \mathrm{CH}_{3} \mathrm{OH}$ in 0.4 bar Helium (300 Pulse), (c) Jetexpansionen von $0.7 \% \mathrm{CH}_{3} \mathrm{OH}$ in 0.8 bar Helium (400 Pulse), (d) berechnetes Methanol-Monomerspektrum für $25 \mathrm{~K}$ und (e) berechnetes Spektrum der Q-Zweige von $\mathrm{CH}_{3} \mathrm{OH}$ für $25 \mathrm{~K}$. Die berechneten Spektren wurden so skaliert, dass die jeweiligen Intensitäten des Q-Zweigs bei $475 \mathrm{~cm}^{-1}$ mit den Intensitäten der experimentellen Spektren übereinstimmen. In den Spektren der Jetexpansionen ergeben sich bei höherem Stagnationsdruck deutlich höhere Intensitäten für die Clusterbanden, die mit einem Sternchen (*) gekennzeichnet sind.

identifizieren, die im Bereich von 530 bis $630 \mathrm{~cm}^{-1}$ bei höherem Druck deutlich höhere Intensität aufweisen und in Abbildung 5.2 mit Sternchen gekennzeichnet sind. Bei genauerer Betrachtung der Jetspektren im Bereich der Monomerfundamentalübergänge $\left(200-350 \mathrm{~cm}^{-1}\right)$ zeigt sich eine etwas bessere Übereinstimmung des Jetspektrums bei geringerem Stagnationsdruck von 
0.4 bar (b) mit dem berechneten Spektrum für eine Temperatur von 50 K (a), während das Spektrum bei höherem Druck von 0.8 bar (c) etwas besser zum berechneten Spektrum für $25 \mathrm{~K}$ (d) passt. Ein solcher Temperatureffekt durch effektivere Kühlung bei höherem Stagnationsdruck ist durchaus möglich. Jedoch ist auch anzumerken, dass keine perfekte Übereinstimmung zwischen den Jetspektren und berechneten Spektren besteht. So ist die Linienliste in Referenz 175 nicht vollständig und es fehlen bei schwachen Banden teilweise die Übergänge aus niedrigen $J$-Zuständen, die bei tiefen Temperaturen jedoch wichtig sind. Vor allem aber sind die Bedingungen in Jetexpansionen fern vom thermischen Gleichgewicht und somit auch der Temperaturbegriff problematisch. Die berechneten Spektren in Abbildung 5.2 sind also eher als Grenzfälle für die Jetspektren zu sehen. Es wurde experimentell ${ }^{216}$ und theoretisch ${ }^{215}$ festgestellt, dass bei Stößen zwischen Methanol und Helium die zur Änderung von $J$ führen, Übergänge mit $\Delta K=0$ deutlich bevorzugt sind gegenüber Übergängen, bei denen sich $K$ ändert. Es könnte also in Jetexpansionen von Methanol in Helium eine wärmere Verteilung auf die $K$-Niveaus vorliegen als auf die jeweiligen $J$-Zustände. Dabei würde sich demnach aus den Intensitäten der Übergänge eine höhere Temperatur ergeben als aus der Form der Rotationszweige. Außerdem wird am Filet-Jet ein relativ großer Ausschnitt der Expansionen gemessen, sodass die Spektren Mittelungen über verschieden warme Regionen entsprechen.

Zusätzlich ist in Abbildung 5.2 ein berechnetes Spektrum (e) gezeigt, das nur auf den Wellenzahlen der Übergänge zwischen den einzelnen $K$-Zuständen beruht und bei dem die Rotationskonturen durch unterschiedliche $J$-Zustände außer Acht gelassen werden. So entsprechen die berechneten Peaks in Spektrum e, die als Gausskurven mit $4 \mathrm{~cm}^{-1}$ Halbwertsbreite dargestellt sind, sehr genau den Positionen der Q-Zweige in den Jet-Spektren. Die relativen Intensitäten beruhen auf der Umrechnung theoretischer Werte für die Q-Zweige von $\mathrm{CH}_{3} \mathrm{OH}$ bei Raumtemperatur $^{218}$ auf $25 \mathrm{~K}$. Sie sind wieder so skaliert, dass die Intensität des Q-Zweigs bei $475 \mathrm{~cm}^{-1}$ mit der experimentellen Intensität übereinstimmt. Trotz der größeren Simplifizierung und nichtexperimenteller Intensitäten ist das berechnete Spektrum e geeignet um die Monomerübergänge in den Jetspektren zu identifizieren. Außerdem entspricht es der physikalisch plausiblen Vorstellung, dass die effektive Temperatur der $J$-Zustände niedriger ist als die effektive Temperatur der $K$-Zustände. Diese Feststellung ist wichtig im Hinblick auf die deuterierten Isotopologen von Methanol für die umfassende Linienlisten nicht existieren. Analoge Monomervorhersagen zum berechneten Spektrum e sind jedoch vorhanden. Für Obertonübergänge sind die Daten zu den theoretischen Intensitäten jedoch nicht vollständig, sodass für diese Übergänge größtenteils Intensitäten bei Raumtemperatur willkürlich festgelegt werden mussten. Die verwendeten Wellenzahlen und Intensitäten finden sich in Tabelle A.2 im Anhang. Diese Methode überschätzt zunehmend die Besetzung hoher $K$-Zustände, da der Einfluss der Quantenzahl $J$ auf die Energien nicht beachtet wird. Außerdem muss näherungsweise angenommen werden, dass die Absorptionskoeffizienten der Q-Zweige nicht von der Temperatur abhängen.

Die Clusterbanden im Bereich von $530-630 \mathrm{~cm}^{-1}$ in Abbildung 5.2 lassen sich zwar durch ihre höhere Intensität bei höherem Druck leicht als solche identifizieren, jedoch ist eine Zuordnung zu unterschiedlichen Clustergrößen nicht möglich, da ihr Wachstumsverhalten bei Druckerhöhung sie nicht ausreichend voneinander diskriminiert und eine eventuelle Überlappung der Banden die Zuordnung ebenfalls erschwert. Es können jedoch zwei intensive Signale bei $610 \mathrm{~cm}^{-1}$ und $551 \mathrm{~cm}^{-1}$ und ein schwächeres Signal bei $567 \mathrm{~cm}^{-1}$ beobachtet werden. Die stärkeren Sig- 


\section{5. $\mathrm{O}-\mathrm{H}$ Torsionen}

nale wurden bereits in der Literatur beschrieben und dem Trimer von $\mathrm{CH}_{3} \mathrm{OH}$ mit Hilfe von quantenchemischen Rechnungen zugeordnet. ${ }^{12,13}$
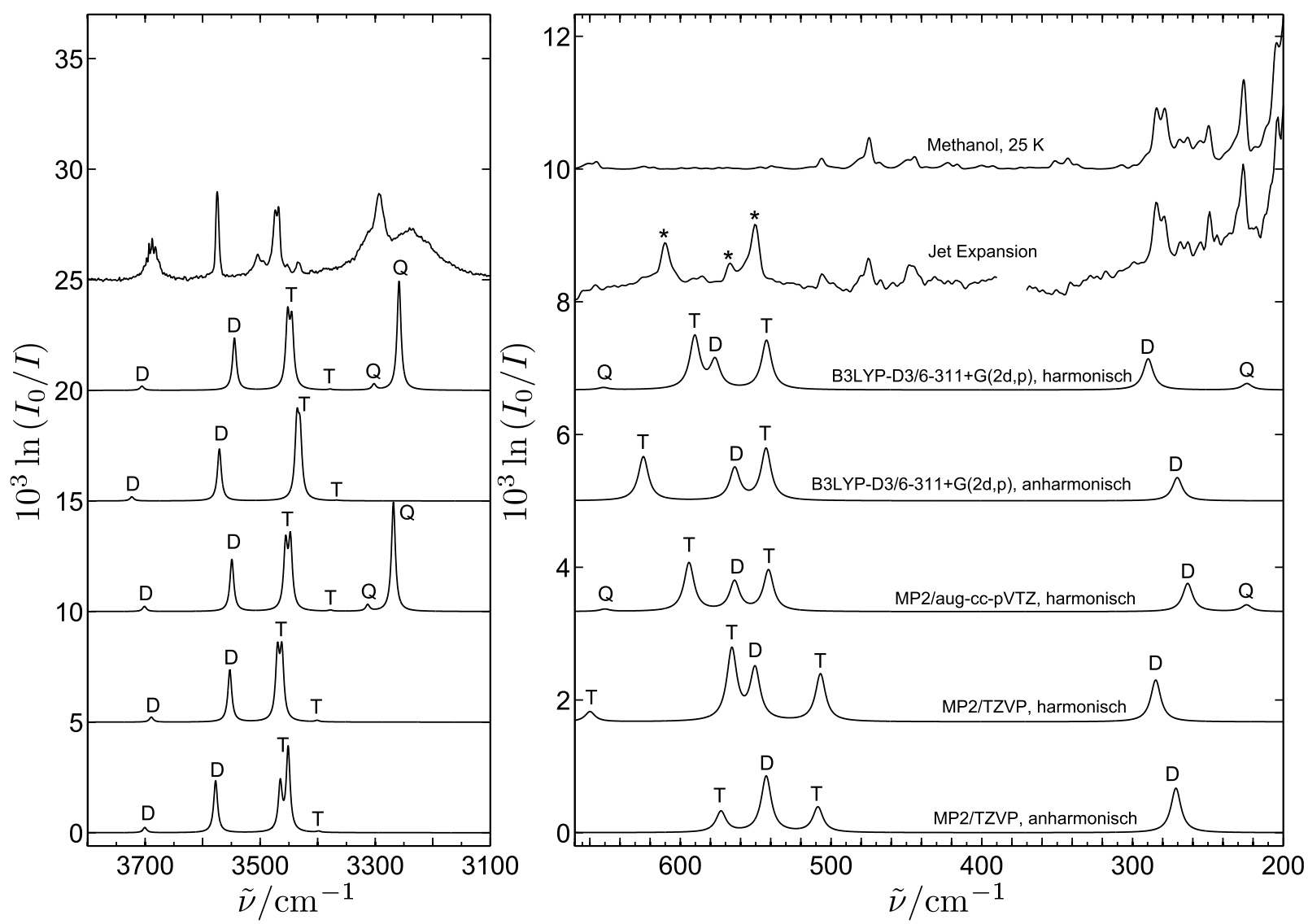

Abbildung 5.3.: Vergleich der Spektren von Jetexpansionen von $0.3 \% \mathrm{CH}_{3} \mathrm{OH}$ in 0.8 bar $\mathrm{He}$ lium im $\mathrm{O}-\mathrm{H}$ Streckbereich (10 Pulse) und Ferninfrarotbereich (400 Pulse) mit quantenchemischen Frequenzvorhersagen zu Methanolclusterfundamentalen. Die Wellenzahlen aus den Rechnungen wurden mit Faktoren skaliert, die sich aus den experimentellen Positionen der Monomer- und mittleren Trimerbande ergeben. Die Intensitäten der einzelnen Clusterspezies im $\mathrm{O}-\mathrm{H}$ Streckbereich sind so skaliert, dass sie mit den experimentellen Intensitäten übereinstimmen. Diese Skalierungen wurden dann für den Ferninfrarotbereich übernommen. Außerdem ist ein berechnetes Monomerspektrum für $25 \mathrm{~K}$ im Ferninfrarotbereich zur leichteren Identifizierung der Monomerübergänge gezeigt. Die Clusterbanden im Jetspektrum sind mit Sternchen gekennzeichnet.

In Abbildung 5.3 sind die Spektren von Jetexpansionen von $0.3 \% \mathrm{CH}_{3} \mathrm{OH}$ in 0.8 bar Helium im $\mathrm{O}-\mathrm{H}$ Streckschwingungsbereich und Ferninfrarotbereich zusammen mit berechneten Spektren der Cluster aus verschiedenen quantenchemischen Ansätzen abgebildet. Diese wurden bis zum Tetramer (Q) oder bis zum Trimer (T) durchgeführt (für Clusterstrukturen siehe Abbildung 2.1). 
Da die Clusterbanden bis zum Tetramer im Bereich der $\mathrm{O}-\mathrm{H}-\mathrm{Streckschwingung}$ bereits zugeordnet sind, ${ }^{36}$ wurde dieser Bereich genutzt um die Konzentration der verschiedenen Clustergrößen mit Hilfe der berechneten Intensitäten abzuschätzen. Dazu wurden die intensivsten Banden der jeweiligen Clustergröße in den Jetspektren der $\mathrm{O}-\mathrm{H}$ Streckschwingung integriert. Die berechneten Intensitäten der Cluster in Abbildung 5.3 wurden dann so skaliert, dass sie mit den Intensitäten im experimentellen Spektrum der $\mathrm{O}-\mathrm{H}$ Streckschwingung übereinstimmen. So ergibt sich durch identische Skalierung eine Vorhersage für die Bandenintensitäten der Cluster im Ferninfrarotbereich unter denselben Expansionsbedingungen. Eine Korrektur für die unterschiedlichen Flächen der Detektoren, die für die Messung der beiden spektralen Bereiche Verwendung fanden, wurde jedoch nicht vorgenommen. Dargestellt sind die berechneten Clusterbanden als Lorentzfunktionen mit $8 \mathrm{~cm}^{-1}$ Halbwertsbreite. Außerdem lassen sich die Konzentrationsverhältnisse der Clusterspezies zueinander anhand der $\mathrm{O}-\mathrm{H}$ Streckschwingungsintensitäten abschätzen. Für das Jetspektrum in Abbildung 5.3 ergibt sich für das Verhältnis Dimer:Trimer:Tetramer 1.0:0.8:0.3 im Messbereich der Jetexpansionen. Die Wellenzahlen der Clusterbanden wurden in den beiden spektralen Fenstern jeweils mit einem Faktor skaliert, der sich aus den Verhältnissen des experimentellen Bandenzentrum des Monomers und der Position der mittleren Trimerbande zu den entsprechenden quantenchemischen Vorhersagen ergibt. Diese Skalierung stellt einen Kompromiss zwischen der Präzision der Vorhersage absoluter Wellenzahlen für gebundene und freie $\mathrm{O}-\mathrm{H}-$ Gruppen dar. Die Beschreibung der $\mathrm{O}-\mathrm{H}$ Torsion des Monomers ist in den quantenchemischen Rechnungen besonders schlecht, da diese nur anhand des Potentials am Minimum berechnet wird. Die Position der mittleren Bande der Trimerlibrationen ist in Referenz 12 gegeben und ihre Zuordnung ist konsistent mit der hier folgenden Analyse. Die berechneten Wellenzahlen und Intensitäten, sowie die jeweiligen Skalierungsfaktoren für den $\mathrm{O}-\mathrm{H}$ Streckschwingungsbereich und Ferninfrarotbereich sind in Tabelle A.6 im Anhang angegeben.

In allen Rechnungen ergeben sich für das Methanoldimer (D) jeweils ähnliche Intensitäten für die Torsion der $\mathrm{O}-\mathrm{H}$ Gruppen der Donor und Akzeptormoleküle. Trimere zeigen zwei IRintensive Librationsbanden mit ähnlicher Intensität und eine höherenergetische Libration mit geringerer Intensität. Die IR-intensivsten Librationsschwingungen der Tetramere liegen außerhalb des spektralen Fensters und wurden experimentell als entartetes Paar bei $760 \mathrm{~cm}^{-1}$ zugeordnet. ${ }^{12,13}$ Auch die IR-schwächere Libration des Tetramers liegt entgegen den Vorhersagen in Abbildung 5.3 außerhalb des spektralen Fensters bei $695 \mathrm{~cm}^{-1} \cdot{ }^{12,13}$

Auf allen Rechenniveaus wird die Position der Fundamentale der Dimerdonorlibration zwischen den beiden intensiven Übergängen der Trimerlibrationen vorhergesagt. Außerdem ergeben sich für die Trimere auf vier Rechenniveaus höhere Intensitäten für die Librationsbanden als für den Übergang des Dimerdonors. Nur in der anharmonischen MP2/TZVP Analyse zeigt der Dimerdonor eine höhere Intensität als die Trimerbanden. Dieser Effekt geht auf die Trimervorhersage auf diesem Niveau zurück, die für die $\mathrm{O}-\mathrm{H}$ Streckschwingungsbanden höhere und für die Librationsbanden niedrigere Intensitäten vorhersagt.

Auf Grundlage der Rechnungen ergibt sich zwar kein absolut konsistentes Bild für die Zuordnung der Clusterbanden des Jetspektrums. Die wahrscheinlichste Zuordnung ist jedoch die beiden intensivsten Banden im Jetspektrum bei $610 \mathrm{~cm}^{-1}$ und $551 \mathrm{~cm}^{-1}$ analog zur Literatur auf das Trimer zurückzuführen, ${ }^{12,13}$ da ihre berechnete Aufspaltung einigermaßen zu dem experimentellen Wert von $59 \mathrm{~cm}^{-1}$ passt und ihre ähnliche Intensität in den Jetspektren konsistent mit 


\section{5. $\mathrm{O}-\mathrm{H}$ Torsionen}

den quantenchemischen Vorhersagen ist. Außerdem sind die vorhergesagten absoluten Intensitäten, außer im Falle der anharmonischen MP2/TZVP Rechnung, in guter Übereinstimmung mit den tatsächlichen Intensitäten aus dem Experiment. Allerdings lässt sich eine teilweise Überlappung der Dimerbande mit den Trimersignalen nicht sicher ausschließen.

Für die Zuordnung der Libration des Dimerdonors verbleibt dennoch das schwächere Signal bei $567 \mathrm{~cm}^{-1}$ als naheliegendste Zuordnung. Passend ist hierfür die Vorhersage, dass dieser Schwingungsübergang zwischen den Trimerübergängen liegt und eine schwächere Intensität im Spektrum zeigen sollte (außer im Falle der anharmonischen MP2/TZVP Rechnung). Weniger passend ist die absolute Intensität der Librationsbande des Dimerdonors, die in den experimentell skalierten Rechnungen konsistent höher vorhergesagt wird als die Bande bei $567 \mathrm{~cm}^{-1}$.

Für die $\mathrm{O}-\mathrm{H}$ Torsion des Dimerakzeptors lässt sich in den Jetspektren kein Übergang finden. Grund dafür kann die Überlagerung mit den Fundamentalübergängen des Monomers sein, da die Position des Dimerakzeptors laut Vorhersage im Bereich von 250 bis $280 \mathrm{~cm}^{-1}$ liegen sollte. Außerdem könnte die Intensität der Dimerakzeptorbande signifikant schwächer gegenüber den Monomerübergängen ausfallen, wenn man wie vorhergesagt ähnliche Intensitäten für Dimerdonor- und -akzeptorbanden annimmt und die mögliche geringe Intensität des Dimerdonors im Spektrum in Betracht zieht.

In Abbildung 5.4 ist ein experimentelles Jetspektrum von $\mathrm{CD}_{3} \mathrm{OH}$ gezeigt. Dazu wurden die Spektren von unterschiedlichen Konzentrationen von $\mathrm{CD}_{3} \mathrm{OH}$ in 0.8 bar Helium gemittelt. Da die Intensitäten von Monomer und Clustern nur wenig von der Konzentration von $\mathrm{CD}_{3} \mathrm{OH}$ abhängen, kann so eine Verbesserung des Signal-zu-Rauschen Verhältnisses ohne signifikante Intensitätsverfälschung erreicht werden. Ein berechnetes Spektrum der Q-Zweige (Gausskurven mit $4 \mathrm{~cm}^{-1}$ Halbwertsbreite), dessen Intensität auf eine möglichst gute Übereinstimmung mit dem Jetspektrum skaliert wurde, zeigt die Übergänge des $\mathrm{CD}_{3} \mathrm{OH}$ Monomer auf. Die verwendeten Daten sind in Tabelle A.3 im Anhang zusammengefasst. Außerdem sind wieder die Vorhersagen quantenchemischer Methoden gezeigt, für die dieselben Skalierungsfaktoren für die Wellenzahlen wie für $\mathrm{CH}_{3} \mathrm{OH}$ in Abbildung 5.3 verwendet und auch die gleichen Konzentrationen der einzelnen Clusterspezies angenommen wurden. Die Effekte der C-Deuterierung können in Abbildung 5.4 direkt entnommen werden, da die Spektren von $\mathrm{CH}_{3} \mathrm{OH}$ in grau ebenfalls abgebildet sind. Die größten Effekte ergeben sich für die Monomerübergänge die generell rotverschoben werden. Für die drei Clusterbanden lassen sich schwächere Effekte finden. Der Übergang bei niedrigster Wellenzahl liegt gegenüber $\mathrm{CH}_{3} \mathrm{OH}$ um etwa $1 \mathrm{~cm}^{-1}$ blauverschoben bei $552 \mathrm{~cm}^{-1}$. Für das schwache Signal bei $567 \mathrm{~cm}^{-1}$ ist keine Veränderung festzustellen. Der größte Effekt der C-Deuterierung ist für die Bande bei der höchsten Wellenzahl zu beobachten. Sie erscheint um etwa $4 \mathrm{~cm}^{-1}$ blauverschoben bei $614 \mathrm{~cm}^{-1}$ und büßt gegenüber $\mathrm{CH}_{3} \mathrm{OH}$ etwa die Hälfte ihrer Intensität ein oder wird erheblich verbreitert. Von den Rechnungen werden diese kleinen Effekte für diese Clusterbanden qualitativ nicht richtig erfasst. Generell werden schwache Rotverschiebungen vorhergesagt, was für harmonische Rechnungen auf Grund des Masseneffekts auch nahe liegt. Neben der anharmonischen B3LYP/6-311+G(2d,p) Rechnung, die für die mittlere Trimerbande eine leichte Blauverschiebung vorhersagt zeigt nur die anharmonische MP2/TZVP Rechnung durchgehend Blauverschiebungen. Letztere sagt auch einen Intensitätseffekt für die Clusterbanden bei $610 / 614 \mathrm{~cm}^{-1}$ voraus - allerdings eine Zunahme der Intensität statt der beobachteten Abnahme durch C-Deuterierung. 


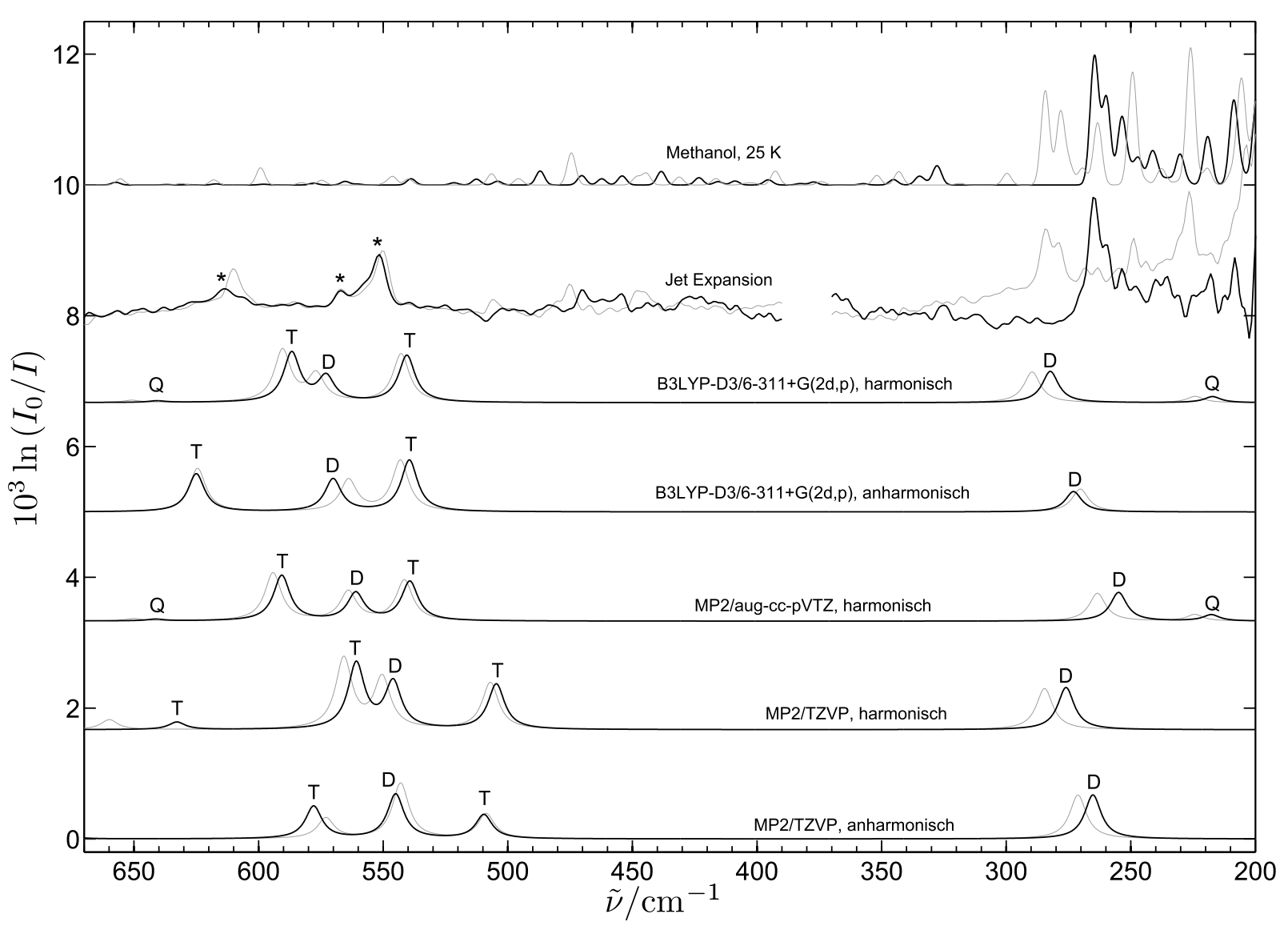

Abbildung 5.4.: Vergleich des Spektrums von Jetexpansionen von $\mathrm{CD}_{3} \mathrm{OH}$ in 0.8 bar Helium mit quantenchemischen Frequenzvorhersagen zu Methanolclustern und vorhergesagtem Monomerspektrum (schwarze Linien). Das experimentelle Spektrum wurde gemittelt aus Expansionen von 0.3\% (90 Pulse) und 0.7\% $\mathrm{CD}_{3} \mathrm{OH}(125$ Pulse). Für die Skalierung der Wellenzahlen und Intensitäten wurden dieselben Faktoren genutzt wie für Abbildung 5.3. Das Monomerspektrum der Q-Zweige bei $25 \mathrm{~K}$ im Ferninfrarotbereich ist zur leichteren Identifizierung der Monomerübergänge gezeigt. Die grauen Linien zeigen die entsprechenden Spektren von $\mathrm{CH}_{3} \mathrm{OH}$ aus Abbildung 5.3 und ein berechnetes Spektrum der Q-Zweige von $\mathrm{CH}_{3} \mathrm{OH}$ Monomer. Da für die Rechnungen identische Faktoren verwendet wurden, gehen die Unterschiede zwischen den Rechnungen zu $\mathrm{CD}_{3} \mathrm{OH}$ und $\mathrm{CH}_{3} \mathrm{OH}$ nicht aus Skalierungseffekten hervor.

Auch für $\mathrm{CD}_{3} \mathrm{OH}$ lässt sich in den Jetspektren kein Signal des Dimerakzeptors finden. Die Rechnungen sagen mit Ausnahme der anharmonischen B3LYP-D3/6-311+G(2d,p) eine Rotverschiebung von 6 bis $8 \mathrm{~cm}^{-1}$ voraus. Grundsätzlich lässt sich zu den Rechenmethoden sagen, dass das anharmonische B3LYP-D3/6-311+G(2d,p) und harmonische MP2/aug-cc-pVTZ Niveau recht gut das Clusterspektrum im Jet vorhersagen. Weniger gut erscheinen die MP2/TZVP 


\section{5. $\mathrm{O}-\mathrm{H}$ Torsionen}

Rechnungen, auf die deshalb in der Analyse zu Methanol-OD verzichtet werden soll.

Matrixisolationsspektroskopie bietet einen weiteren Ansatz um Methanoldimere im Ferninfrarotbereich zu beobachten. So wurde in $\mathrm{N}_{2}$ Matrizen die Libration des Dimerdonors des intensivsten $\mathrm{CH}_{3} \mathrm{OH}$ Dimerkonformers bei $596.1 \mathrm{~cm}^{-1}$ gefunden. ${ }^{172}$ Näher an den Gasphasenwerten sollten jedoch die Wellenzahlen aus Neon-Matrizen sein, die geringere Matrixeffekte aufweisen sollten. In Neon-Matrizen ${ }^{14}$ liegt die $\mathrm{O}-\mathrm{H}$ Libration des $\mathrm{CH}_{3} \mathrm{OH}$ Dimerdonors bei $557 \mathrm{~cm}^{-1}$. Somit liegt dieser Übergang genau zwischen den Banden des Methanoltrimers bei $551 \mathrm{~cm}^{-1}$ und der weniger intensiven Bande bei $567 \mathrm{~cm}^{-1}$ in den Jetexpansionsspektren. Durch C-Deuterierung wird die Donorlibrationsbande in der Ne Matrix um etwa $1 \mathrm{~cm}^{-1}$ blauverschoben, wie es auch für die Banden in Jetexpansionen bei $551 / 552 \mathrm{~cm}^{-1}$ der Fall ist. ${ }^{219}$ Dies zeigt, dass die Torsion der $\mathrm{O}-\mathrm{H}$ Gruppe des Dimerdonors weitgehend entkoppelt ist von der Torsion der Methylgruppen. Der $\mathrm{O}-\mathrm{H}$ Torsionsübergang des $\mathrm{CH}_{3} \mathrm{OH}$ Dimerakzeptors liegt in der Ne-Matrix bei $284 \mathrm{~cm}^{-1}$ und wäre bei gleicher Position im Jet vom $\mathrm{CH}_{3} \mathrm{OH}$ Monomer überdeckt. Interessant ist auch die Verschiebung der Dimerakzeptorbande zur Monomertorsion. Letztere lässt sich aus den Torsionsübergängen des Methanol Monomer in Ne Matrizen ${ }^{176}$ berechnen. Dabei ergibt sich unter zweifacher Gewichtung der $\mathrm{E} \leftrightarrow \mathrm{E}$ Übergänge ein Bandenzentrum für $\mathrm{CH}_{3} \mathrm{OH}-$ Monomer bei $227 \mathrm{~cm}^{-1}$, also eine etwas kleinere Wellenzahl als in der Gasphase für diese Bewegung mit großer Amplitude (Vgl. Tabelle A.2). Der $\mathrm{CH}_{3} \mathrm{OH}$ Dimerakzeptor ist in Ne Matrizen somit um $59 \mathrm{~cm}^{-1}$ deutlich blauverschoben zum Monomer. 


\subsubsection{Methanol-OD}

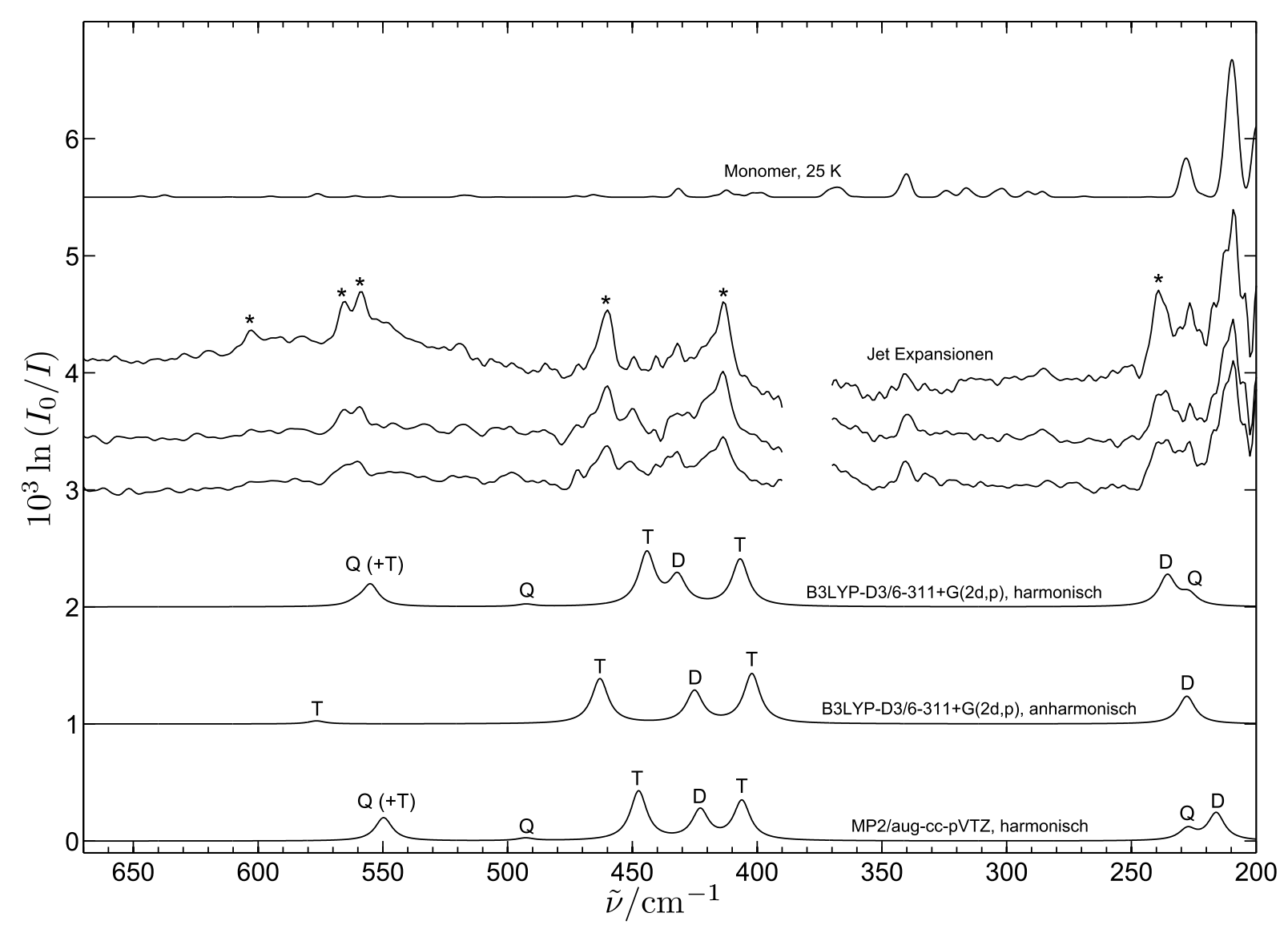

Abbildung 5.5.: Vergleich der Spektren von Jetexpansionen von $\mathrm{CH}_{3} \mathrm{OD}$ mit quantenchemischen Frequenzvorhersagen zu Methanolclustern. Die experimentellen Spektren entsprechen von unten nach oben $0.6 \% \mathrm{CH}_{3} \mathrm{OD}$ in 0.4 bar Helium, $0.3 \% \mathrm{CH}_{3} \mathrm{OD}$ in 0.8 bar Helium und $0.7 \% \mathrm{CH}_{3} \mathrm{OD}$ in 0.8 bar Helium (jeweils 400 Pulse). Die Wellenzahlen aus den Rechnungen wurden mit Faktoren skaliert, die sich aus den experimentellen Positionen der Monomer- und mittleren Trimerbande ergeben. Die Intensitäten wurden für die gleiche Clusterkonzentrationen wie in Abbildung 5.3 berechnet, die den Expansionsbedingungen des hier gezeigten mittleren Jetspektrums entsprechen. Außerdem ist ein berechnetes Spektrum der Q-Zweige von $\mathrm{CH}_{3} \mathrm{OD}$ Monomer abgebildet.

Die Torsionsübergänge der O-D Gruppe liegen für Monomer und Cluster bei deutlich niedrigeren Wellenzahlen und auch die Intensitäten nehmen im Vergleich zum undeuterierten Methanol ab. Das resultierende schlechtere Signal-zu-Rauschen Verhältnis erschwert die Zuordnung der Spektren. In Abbildung 5.5 sind die Jetspektren von $\mathrm{CH}_{3} \mathrm{OD}$ abgebildet. Die Banden des Monomers können aus dem Vergleich mit dem berechneten Spektrum der Q-Zweige von $\mathrm{CH}_{3} \mathrm{OD}$ bei $25 \mathrm{~K}$ identifiziert werden, dessen Wellenzahlen und Intensitäten in Tabelle A.4 im Anhang 


\section{5. $\mathrm{O}-\mathrm{H}$ Torsionen}

gegeben sind. Einige Clusterbanden lassen sich anhand ihres Wachstumsverhaltens bei höherem Druck und höherer $\mathrm{CH}_{3} \mathrm{OD}$ Konzentration identifizieren und sind mit Sternchen gekennzeichnet. Wieder lassen sich unter anderem zwei intensive Clustersignale bei $414 \mathrm{~cm}^{-1}$ und $460 \mathrm{~cm}^{-1}$ beobachten, für deren Ursprung die Übergänge der Methanoltrimerlibrationen angenommen werden können. So kann die höherenergetische Bande wieder zusammen mit der Monomerposition zur Skalierung der berechneten Wellenzahlen der Clusterbanden, analog zu Abbildung 5.3, genutzt werden. Die Intensitäten der berechneten Übergänge in Abbildung 5.5 wurden zum Vergleich mit dem mittleren Jetspektrum skaliert. Dieses Spektrum wurde unter den gleichen Expansionsbedingungen wie das $\mathrm{CH}_{3} \mathrm{OH}$ Spektrum in Abbildung 5.3 aufgenommen, sodass für $\mathrm{CH}_{3} \mathrm{OD}$ die gleichen Konzentrationen der Clusterspezies angenommen werden können.

Durch die niedrigeren Schwingungsenergien der O-D Torsion liegen auch Librationsübergänge des Methanoltetramers innerhalb des spektroskopischen Fensters von 200 bis $670 \mathrm{~cm}^{-1}$. Für sie wird ein entartetes Paar von Übergängen mit hoher Intensität vorausgesagt, denen in den Spektren die Banden bei $559 \mathrm{~cm}^{-1}$ und $566 \mathrm{~cm}^{-1}$ zugeordnet werden können. Dieses Dublett geht wahrscheinlich auf eine Fermiresonanz zurück, wie hier anschließend durch die Spektren von volldeuteriertem Methanol gezeigt werden kann. Ein schwächerer Librationsübergang des Tetramers wird bei niedrigerer Wellenzahl erwartet, jedoch könnte seine Intensität zu gering sein, um ihn zu beobachten. Eine mögliche Position wäre ein schwaches Signal bei $519 \mathrm{~cm}^{-1}$, jedoch liegt an dieser Stelle ebenfalls ein Monomerübergang. Ein weiteres Clustersignal bei $603 \mathrm{~cm}^{-1}$ lässt sich anhand der Rechnungen nicht zuordnen. Möglich wäre, dass es sich hierbei um einen Übergang größerer Cluster wie dem Pentamer von Methanol handelt.

Die Libration des Dimerdonors lässt sich für $\mathrm{CH}_{3} \mathrm{OD}$ anhand der Jetspektren nicht zuordnen. Von den quantenchemischen Rechnungen wird der Übergang wieder zwischen den intensiven Trimersignalen vorhergesagt. In diesem Bereich liegen jedoch auch einige Monomerübergänge und das schlechte Signal-zu-Rauschen Verhältnis lässt das identifizieren weiterer Clusterbanden nicht zu. In Ne-Matrizen liegt der Librationsübergang des $\mathrm{CH}_{3} \mathrm{OD}$ Dimers bei $420 \mathrm{~cm}^{-1} \cdot{ }^{14}$ Dies entspricht mit $6 \mathrm{~cm}^{-1}$ derselben Verschiebung zu der tiefsten Trimerlibrationsbande in den Jetspektren, wie im Falle der $\mathrm{CH}_{3} \mathrm{OH}$ Signale in den beiden experimentellen Ansätzen. Dies spricht dafür, dass der Dimerdonorübergang in Jetexpansionen relativ zur längerwelligen Bande des Trimers für $\mathrm{CH}_{3} \mathrm{OH}$ und $\mathrm{CH}_{3} \mathrm{OD}$ eine ähnliche Position einnehmen sollte und möglicherweise mit ihr deutlich überlappt.

Für die O-D Torsion des Dimerakzeptors findet sich jedoch ein mögliches Signal in den Jetspektren. Bei $238 \mathrm{~cm}^{-1}$ liegt ein Signal dessen Intensität in etwa den intensiveren Clusterbanden entspricht. Da an dieser Position kein Q-Zweig des Monomers erwartet wird, ist die Zuordnung zu Clustern wahrscheinlicher, als die Annahme intensiver R-Zweige der Monomerbanden bei 229 und $227 \mathrm{~cm}^{-1}$. Auch eine Verunreinigung durch $\mathrm{CH}_{3} \mathrm{OH}$ kann ausgeschlossen werden, da dieses an dieser Position keine intensiven Banden zeigt und auch sonst keine Signale von $\mathrm{CH}_{3} \mathrm{OH}$ in den Jetspektren beobachtet werden. In Neonmatrizen liegt der Torsionsübergang des Dimerakzeptors in ähnlicher Position bei $234 \mathrm{~cm}^{-1} .{ }^{14}$ Somit ist die Zuordnung der Bande bei $238 \mathrm{~cm}^{-1}$ zum Dimerakzeptor insgesamt konsistent. Nur die hohe Intensität des Übergangs deutet auf eventuelle Überlappung mit Monomerübergängen hin.

In Abbildung 5.6 sind die Spektren von $\mathrm{CD}_{3} \mathrm{OD}$ in Jetexpansionen gezeigt. Die Effekte durch die Deuteriumatome am Kohlenstoff auf die Schwingungen der gebundenen O-D Gruppen ist 


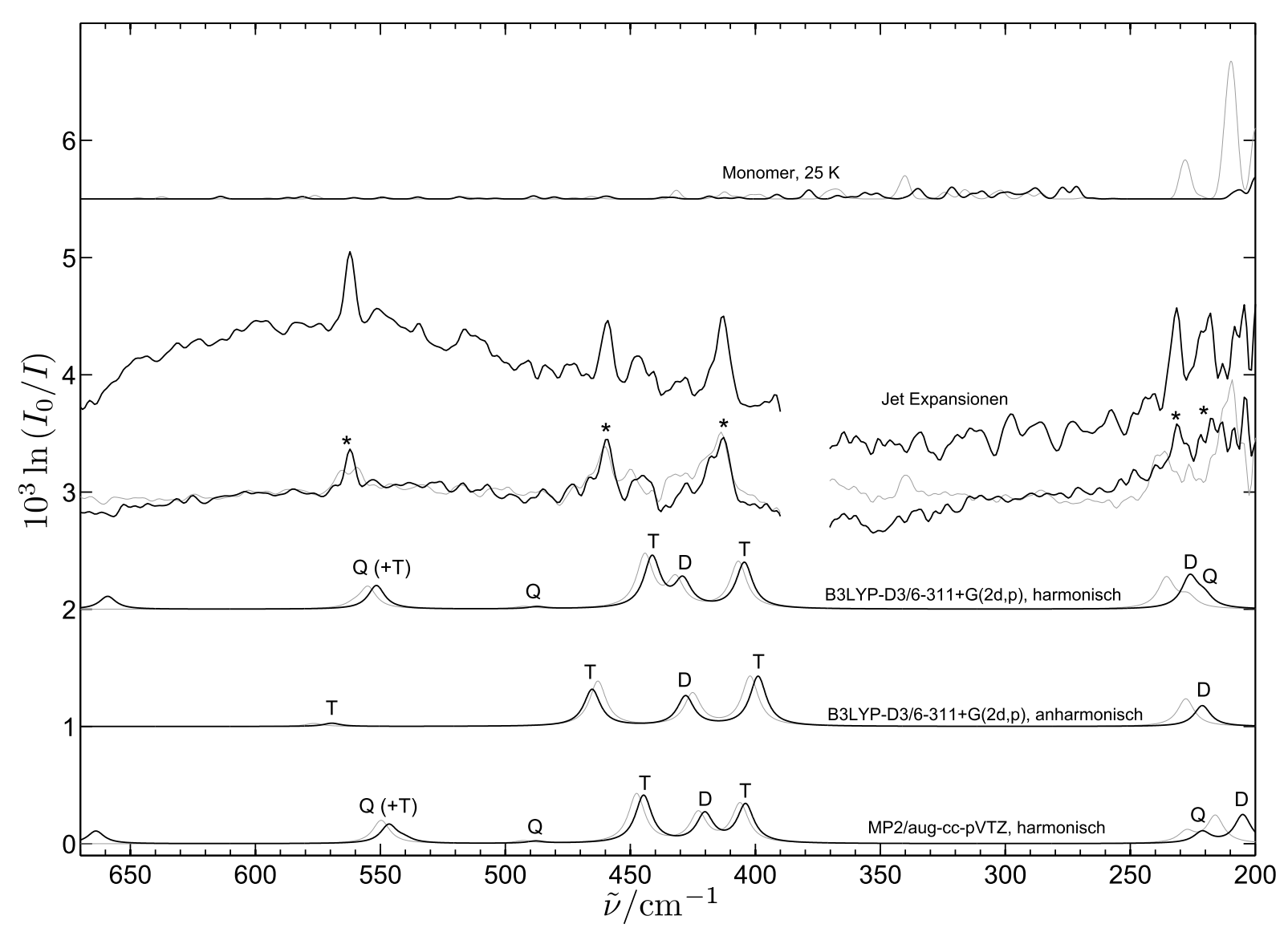

Abbildung 5.6.: Vergleich der Spektren von Jetexpansionen von $\mathrm{CD}_{3} \mathrm{OD}$ mit quantenchemischen Frequenzvorhersagen zu Methanolclustern und vorhergesagtem Monomerspektrum (schwarze Linien). Für die experimentellen Jetspektren wurden $0.3 \%$ (unten, 200 Pulse) und $0.7 \% \mathrm{CD}_{3} \mathrm{OD}$ (oben, 125 Pulse) in 0.8 bar $\mathrm{He}-$ lium verwendet. Für die Skalierung der Wellenzahlen wurden dieselben Faktoren genutzt wie in Abbildung 5.5 und die Intensitäten wurden für den Vergleich mit dem unteren Jetspektrum skaliert, wofür die gleiche Konzentration der Cluster wie in Abbildung 5.3 angenommen wurden. Das Monomerspektrum der Q-Zweige bei $25 \mathrm{~K}$ im Ferninfrarotbereich ist zur leichteren Identifizierung der Monomerübergänge gezeigt. Die grauen Linien zeigen die entsprechenden Spektren von $\mathrm{CH}_{3} \mathrm{OD}$ aus Abbildung 5.5 und ein berechnetes Spektrum der QZweige von $\mathrm{CH}_{3} \mathrm{OD}$ Monomer. Da für die Rechnungen identische Faktoren verwendet wurden, gehen die Unterschiede zwischen den Rechnungen zu $\mathrm{CD}_{3} \mathrm{OD}$ und $\mathrm{CH}_{3} \mathrm{OD}$ nicht aus Skalierungseffekten hervor.

gering. Jedoch zeigt sich nun eine einzelne Bande bei $562 \mathrm{~cm}^{-1}$ die dem entarteten Paar von intensiven Librationsübergängen des Tetramers zugeordnet werden kann. Im Falle von $\mathrm{CH}_{3} \mathrm{OD}$ liegt diese Bande als Dublett vor, was die Vermutung einer Fermiresonanz im $\mathrm{CH}_{3} \mathrm{OD}$ Tetramer 


\section{5. $\mathrm{O}-\mathrm{H}$ Torsionen}

nahe legt. Eine plausible Möglichkeit wäre die Resonanz mit einer Kombinationsbande aus dem Librationsübergang bei niedrigster Wellenzahl $\left(\approx 519 \mathrm{~cm}^{-1}\right.$ s.o. $)$ von $\left(\mathrm{CH}_{3} \mathrm{OD}\right)_{4}$ und aus einer zwischenmolekularen Schwingung die einen signifikanten Anteil von Methyltorsion enthält. So würde sich der deutliche Isotopeneffekt durch C-Deuterierung erklären, außerdem kann eine starke Kopplung zwischen $\mathrm{O}-\mathrm{H}$-Torsion und Methyltorsion als Voraussetzung für die Resonanz angenommen werden. Die weniger intensive Librationsbande von $\left(\mathrm{CD}_{3} \mathrm{OD}\right)_{4}$ könnte ungefähr bei $513 \mathrm{~cm}^{-1}$ liegen, jedoch ist eine Überlappung mit Monomerbanden oder Signalen anderer Cluster möglich.

Die intensiven Librationsbanden des $\mathrm{CD}_{3} \mathrm{OD}$ Trimers liegen bei $460 \mathrm{~cm}^{-1}$ und $413 \mathrm{~cm}^{-1}$, letztere damit um $1 \mathrm{~cm}^{-1}$ rotverschoben zum entsprechenden Übergang von $\mathrm{CH}_{3} \mathrm{OD}$. Außerdem findet sich im Bereich der Trimerübergänge ein Signal bei $445 \mathrm{~cm}^{-1}$ das sich nicht eindeutig zuordnen oder als Clusterbande anhand seiner Konzentrationsabhängigkeit charakterisieren lässt. Intensive Monomerbanden werden an dieser Position jedoch nicht erwartet.

Durch die vollständige Deuterierung liegen die intensiven Monomerübergänge außerhalb des spektralen Fensters. Im niederenergetischen Bereich lassen sich jedoch zwei intensive Banden beobachten, die von Clustern zu stammen scheinen. Sie liegen ungefähr bei 231 und $216 \mathrm{~cm}^{-1}$. Möglich wäre, dass sie auf die O-D Torsion des Dimerakzeptors und eine zwischenmolekulare Schwingung des Tetramers zurückgehen. Die vorhergesagten Intensitäten dieser Schwingungen sind mit diesen möglichen Zuordnungen konsistent.

\subsubsection{Zusammenfassung}

Da die Expansionen von Methanol am Filet-Jet keine Größenselektivität bieten, werden bei hohen Clusterkonzentrationen viele verschiedene Clustergrößen gleichzeitig gemessen. Dabei ist die Konzentrationsabhängigkeit der Bandenintensitäten bei Ferninfrarotexperimenten nicht ausreichend um die einzelnen Clusterspezies von Methanol experimentell im Spektrum zu identifizieren. Jedoch gelingt die Unterscheidung von Monomerübergängen durch Variation des Stagnationsdrucks und der Methanolkonzentration. Auch die Vorhersage der Übergänge der kalten Monomere hilft bei der Identifikation von Clusterbanden.

Die Zuordnung der Clustersignale zu den verschiedenen Clusterspezies wird durch den Vergleich mit quantenchemischen Frequenzvorhersagen ermöglicht. Dies funktioniert gut für die intensiven Signale der Tetramere und Trimere. Die Libration der Wasserstoffbrückendonoren des Methanoldimers konnte nicht zugeordnet werden. Grund dafür ist eine eventuelle Überlappung mit Trimersignalen, eine geringe spektrale Intensität oder die Aufspaltung in mehrere Peaks. Möglich ist, dass die komplexe Tunneldynamik und Schwingungsrotationskopplung des Monomers auch noch im Dimer zu finden ist. So ist zwar die Aufspaltung im Schwingungsgrundzustand durch Tunneln der $\mathrm{O}-\mathrm{H}$ Gruppe kleiner als $0.05 \mathrm{~cm}^{-1}$ und kleiner als $1 \mathrm{~cm}^{-1}$ durch Tunneln bei der inneren Torsion der Methylgruppen, ${ }^{220}$ jedoch führen die Schwingungskoordinaten der Libration des Dimerdonors entlang der Trajektorie, die den Wasserstoffbrückendonor in den Akzeptor überführt und umgekehrt. Somit könnte durchaus im angeregten Librationszustand eine höhere Tunnelaufspaltung vorliegen. Durch Deuterierung der Alkoholfunktionalität sollten diese Effekte verringert werden. Auf Grund der schwachen Signale besonders der O-deuterierten Spezies lassen sich jedoch keine Aussagen über eventuelle Tunnelaufspaltungen der Dimerlibra- 
Tabelle 5.3.: Experimentelle Wellenzahlen der intensivsten $\mathrm{O}-\mathrm{H}$ bzw. O-D Torsionsübergänge von Methanolisotopologen in verschiedenen Umgebungen und Clustergrößen. Außerdem ist das Verhältnis der Wellenzahlen $\frac{\tilde{\nu}_{\mathrm{OH}}}{\tilde{\nu}_{\mathrm{OD}}}$ zwischen den Schwingungen der $\mathrm{CH}_{3} \mathrm{OH}$ und $\mathrm{CH}_{3} \mathrm{OD}$ Spezies gegeben.

\begin{tabular}{|c|c|c|c|c|c|}
\hline & $\begin{array}{l}\tilde{\nu} / \mathrm{cm}^{-1} \\
\mathrm{CH}_{3} \mathrm{OH}\end{array}$ & $\begin{array}{l}\tilde{\nu} / \mathrm{cm}^{-1} \\
\mathrm{CD}_{3} \mathrm{OH}\end{array}$ & $\begin{array}{l}\tilde{\nu} / \mathrm{cm}^{-1} \\
\mathrm{CH}_{3} \mathrm{OD}\end{array}$ & $\begin{array}{l}\tilde{\nu} / \mathrm{cm}^{-1} \\
\mathrm{CD}_{3} \mathrm{OD}\end{array}$ & $\frac{\tilde{\nu}_{\mathrm{OH}}}{\tilde{\nu}_{\mathrm{OD}}}$ \\
\hline \multicolumn{6}{|c|}{ Gasphase } \\
\hline Monomer & 231.3 & 220.0 & 189.8 & 176.8 & 1.22 \\
\hline \multicolumn{6}{|c|}{ Ne-Matrix } \\
\hline \multirow[t]{2}{*}{ Dimer } & 284 & & 234 & & 1.21 \\
\hline & 557 & 558 & 420 & & 1.33 \\
\hline \multicolumn{6}{|c|}{ He-Expansionen } \\
\hline \multirow[t]{2}{*}{ Trimer } & 551 & 552 & 414 & 413 & 1.33 \\
\hline & 610 & 614 & 460 & 460 & 1.33 \\
\hline \multirow[t]{2}{*}{ Tetramer } & $695^{a}$ & & [519] & {$[\approx 513]$} & 1.34 \\
\hline & $760^{a}$ & & $559 / 566$ & 562 & $1.36 / 1.34$ \\
\hline
\end{tabular}

${ }^{a}$ Referenz 13.

tionsbande treffen. Für den Akzeptor wurden im Falle der O-deuterierten Spezies Banden für eine mögliche Zuordnung gefunden. Der Grund dafür könnte der zuvor beschriebene sein: Eine Tunneldynamik der $\mathrm{O}-\mathrm{H}$ Spezies, die im Falle von O-D abgeschwächt wird. Außerdem könnte auch die höhere Masse des Deuteriums, die zu einer geringeren Amplitude der Schwingung führt, zu deutlicheren Signalen im Spektrum führen.

In Tabelle 5.3 sind die Wellenzahlen der $\mathrm{O}-\mathrm{H}$ oder $\mathrm{O}-\mathrm{D}$ Torsionsübergänge der Methanolisotopologen vom Monomer bis Tetramer aus verschiedenen experimentellen Ansätzen zusammengefasst. Interessant dabei ist auch das Verhältnis der Wellenzahlen der $\mathrm{CH}_{3} \mathrm{OH}$ Spezies zu $\mathrm{CH}_{3} \mathrm{OD}$. Diese Verhältnisse sind abhängig von den Normalkoordinaten und Anharmonizitäten der Schwingungen. Dabei zeigen sich für die Verhältnisse der Hydroxyltorsion deutliche Unterschiede zwischen den freien $(1.21-1.22)$ und den in Wasserstoffbrücken gebundenen Gruppen $(1.33-1.36)$.

Es zeigt sich, dass Messungen in Edelgasmatrizen, insbesondere in Neon, geeignet sind um die Librationsbanden vom Methanoldimer zu untersuchen. Die Bandenpositionen in Neonmatrizen, die vermutlich nur wenig von den Gasphasenwerten abweichen, stellen eine wichtige Hilfe dar, um die Jetspektren von Methanol zu interpretieren. 


\section{3. tert-Butylalkohol}

\subsection{1. tert-Butylalkohol-OH}

Im spektroskopischen Fenster von $200-670 \mathrm{~cm}^{-1}$ liegen mehrere Übergänge von tert-Butylalkohol, deren Signale in den Spektren von Heliumexpansionen in Abbildung 5.7 sichtbar sind. Die Monomerübergänge und Clusterbanden lassen sich auf Grund der Abhängigkeit ihrer Intensitäten von tert-Butylalkoholkonzentration und Stagnationsdruck differenzieren. Außerdem hilft ein Spektrum von tert-Butylalkohol in einer Neonmatrix, das von René Wugt Larsen zur Verfügung gestellt wurde, ${ }^{219}$ die zu erwartenden Bandenpositionen der Monomere zu markieren. Die Strukturen der tert-Butylalkoholcluster sind in Abbildung 2.1 in Kapitel 2.2 abgebildet.

Im Schwingungsgrundzustand führt das Torsionspotential der O-H Gruppe zu einer Aufspaltung in einen A und zwei E Zustände, ${ }^{221}$ deren experimenteller Wert bisher nicht bekannt ist. In quantenchemischen Rechnungen werden zwei $\mathrm{O}-\mathrm{H}$ Torsionsübergänge für das tert-Butylalkohol Monomer vorhergesagt, die sich aus unterschiedlicher Kombination mit Rotationen der Methylgruppen ergeben. Somit wären mehrere Torsionsfundamentalen für tert-Butylalkohol zu erwarten, entsprechend den möglichen $\mathrm{A} \leftrightarrow \mathrm{A}$ und $\mathrm{E} \leftrightarrow \mathrm{E}$ Übergängen für die zwei Torsionsschwingungen. In Tabelle 5.4 sind die Bandenpositionen von tert-Butylalkohol in verschiedenen Umgebungen für den Ferninfrarotbereich aufgelistet. In Jetexpansionen und Ne-Matrizen werden tatsächlich vier Monomerbanden im Bereich der OH-Torsion beobachtet. Dies ließe sich mit den je zwei Torsionsübergängen der E- und A-Tunnelspezies erklären, wenn für die angeregten $\mathrm{Zu}$ stände eine erhöhte Tunnelaufspaltung angenommen wird. Für die Spektren in Neon-Matrizen würde wie im Falle von Methanol ${ }^{14}$ außerdem eine Feinaufspaltung aller Banden zu erwarten sein, die auf die Tunnelaufspaltung des Grundzustands zurückzuführen wäre und auf Grund der Aufhebung der Auswahlregeln zum Erhalt der Tunnelsymmetrie sichtbar würden. Tatsächlich lassen sich Anzeichen für eine solche feine Aufspaltung in den Matrixspektren in Abbildung 5.7 finden. Weitere intensive Übergänge des tert-Butylalkohol Monomers sind die Knickschwingungen der schwereren Atome bei $341 \mathrm{~cm}^{-1}$ und $465 \mathrm{~cm}^{-1}$.

Mehrere Clusterbanden lassen sich in den Jetspektren auf Grund ihres stärkeren Intensitätswachstums mit höherem Druck und tert-Butylalkoholkonzentration identifizieren. Außerdem ist es teilweise möglich sie unterschiedlichen Clusterspezies zuzuordnen. Die Zuordnung des $\mathrm{O}-\mathrm{H}$ Streckschwingungsbereichs von tert-Butylalkoholclustern wurde bereits vorgenommen. ${ }^{37} \mathrm{Die}$ zwei oberen Jetspektren in Abbildung 5.7 zeigen im $\mathrm{O}-\mathrm{H}$ Streckschwingungsbereich fast nur Monomer- und Dimersignale, letztere steigern ihre Intensität um einen Faktor 2 bis 3 durch die Erhöhung der tert-Butylalkoholkonzentration. Unter gleichen Bedingungen zeigt sich im Ferninfrarotbereich eine schwache Clusterbande bei $614 \mathrm{~cm}^{-1}$ die somit wahrscheinlich auf das Dimer zurückgeht. Bei weiterer Erhöhung von Druck und Substanzkonzentration wächst die Intensität dieser Bande weiter und es lässt sich eine weitere Clusterbande bei $290 \mathrm{~cm}^{-1}$ identifizieren, die ein sehr ähnliches Wachstumsverhalten zeigt und somit wahrscheinlich auch zum Dimer gehört. Dagegen zeigt sich bei $565 \mathrm{~cm}^{-1}$ eine Bande, die in den Spektren erst bei höherem Druck erscheint, dann aber mit Druck- und Konzentrationserhöhung schneller wächst als die zwei zuvor genannten Banden. Somit ist diese Bande größeren Clustern zuzuordnen. Ein Vergleich mit den Signalen im $\mathrm{O}-\mathrm{H}$ Streckschwingungsbereich zeigt, dass eine Zuordnung zum Trimer auf 

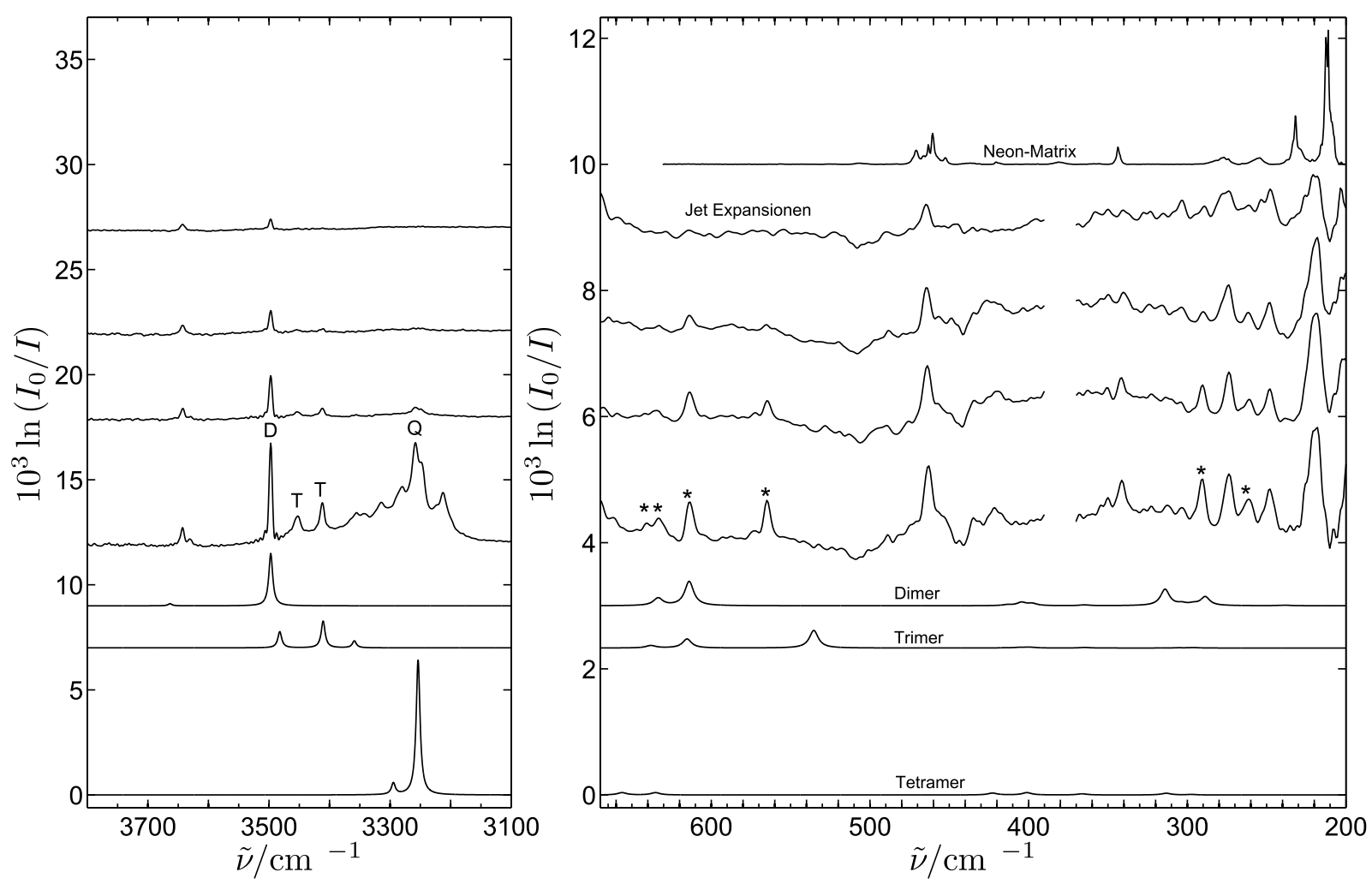

Abbildung 5.7.: Vergleich der Spektren von tert-Butylalkohol in Jetexpansionen und in einer Neon Matrix ${ }^{219}$ mit quantenchemischen Frequenzvorhersagen zu tertButylalkoholclustern. Die Jetspektren wurden im O-H Streckschwingungsbereich und Ferninfrarotbereich aufgenommen. Die Spektren in der Neonmatrix sind nur im Ferninfraroten gezeigt und so skaliert, dass die Banden um $460 \mathrm{~cm}^{-1}$ mit der Intensität des oberen Jetspektrums etwa übereinstimmen. In den experimentellen Jetspektren steigt die Clusterkonzentration in beiden Spektrenfenstern von oben nach unten: $0.06 \%$ tert-Butylalkohol in 0.4 bar Helium (oben, 20 Pulse, 200 Pulse im FIR), 0.09\% tert-Butylalkohol in 0.4 bar Helium (zweites von oben, 10 Pulse, 200 Pulse im FIR), $0.09 \%$ in 0.6 bar Helium (drittes von oben, 20 Pulse, 195 Pulse im FIR), 0.16\% tert-Butylalkohol in 0.8 bar Helium (unten, 20 Pulse, 250 Pulse im FIR). Die Wellenzahlen der harmonischen Schwingungsrechnungen (B3LYP-D3/6-311+G(2d,p)) wurden in beiden Spektralbereichen mit je einem Faktor skaliert, der die intensiven Dimerdonorbanden aus Experiment und Rechnung in Übereinstimmung bringt. Die berechneten Intensitäten wurden für jede Clustergröße so skaliert, dass die experimentellen integrierten Intensitäten im unteren $\mathrm{O}-\mathrm{H}$ Streckschwingungsspektrum mit den berechneten übereinstimmen und sich somit eine Intensitätsvorhersage für das untere Ferninfrarotspektrum ergibt. Experimentell gefundene Clusterbanden sind im Ferninfrarotbereich mit Sternchen markiert. 
Tabelle 5.4.: Bandenpositionen in $\mathrm{cm}^{-1}$ von tert-Butylalkohol im Ferninfrarotbereich: Experimentelle Ergebnisse in verschiedenen Umgebungen und harmonische Vorhersagen auf B3LYP-D3/6-311+G(2d,p) Niveau, für die auch Intensitäten in $\frac{\mathrm{km}}{\mathrm{mol}}$ in Klammern gegeben sind. Die Zuordnung der Normalmoden beruht auf der quantenchemischen Vorhersage. Sie stimmen im Wesentlichen mit den Zuordnungen in Referenz 222 überein. Nur die Methyltorsion sollte in Absorptionsexperimenten nicht sichtbar sein und entsprechend sollte den Signalen bei $272-277 \mathrm{~cm}^{-1}$ die O-HTorsion zugeordnet werden.

\begin{tabular}{lccccc}
\hline & $\begin{array}{c}\text { B3LYP-D3 } \\
\text { /6-311+G(2d,p) }\end{array}$ & $\begin{array}{c}\text { He } \\
\text { Jetexpansion }\end{array}$ & $\begin{array}{c}\text { RT } \\
\text { Gasphase }\end{array}$ & Ne-Matrix 219 & Ar-Matrix ${ }^{222}$ \\
\hline O-H-Torsion & $267(31)$ & $\approx 201$ & & $\approx 212$ & 222.5 \\
& $294(75)$ & 219 & 216.5 & $\approx 231$ & 226.9 \\
& & 248 & & $\approx 255$ & \\
Methyl-Torsion & $213(3.7)$ & 274 & 272.3 & $\approx 277$ & 275.7 \\
& $281(0.1)$ & & & & \\
C-C-C Knick & $349(5.5)$ & 341 & 340.8 & 343 & 344.0 \\
& $350(1.5)$ & & & & \\
symm. C-C-O Knick & $429(0.6)$ & & 419.5 & 420 & 418.3 \\
asymm. C-C-O Knick & $471(8.2)$ & 465 & 462.5 & $452-471$ & 456.0 \\
& $471(7)$ & & & & 461 \\
\hline
\end{tabular}

Grund des Wachstumsverhaltens besser als zum Tetramer passt. Außerdem ist noch mindestens eine Clusterbande im Bereich von 632 bis $642 \mathrm{~cm}^{-1}$ zu identifizieren, die jedoch auf Grund ihrer geringen Intensität keiner genauen Clusterspezies zugeordnet werden kann. Eine weitere Bande bei $261 \mathrm{~cm}^{-1}$ ist ebenfalls schwierig zuzuordnen. Das Wachstumsverhalten ihrer Intensität deutet auf einen Clusterursprung hin, jedoch stellt es sich wegen der geringen Intensität und eventueller Überlappung mit Monomerintensität weniger deutlich dar als im Falle der anderen Banden.

In Neon-Matrizen kann ein breites Signal von 605 bis $620 \mathrm{~cm}^{-1}$ der Libration des Dimerdonors von tert-Butylalkohol zugeordnet werden. ${ }^{219}$ Der Torsionsübergang des Dimerakzeptors kann als ebenfalls breite Bande bei $291 \mathrm{~cm}^{-1}$ beobachtet werden. ${ }^{219}$ Diese Wellenzahlen passen gut zu den beobachteten Übergängen am Filet-Jet und sprechen für die Zuordnung der Dimerschwingungen zu den Banden bei $614 \mathrm{~cm}^{-1}$ und $290 \mathrm{~cm}^{-1}$ in Jetexpansionen.

Weitere Hilfe bei der Zuordnung der Clusterbanden bieten die harmonischen Frequenzvorhersagen auf B3LYP-D3/6-311+G(2d,p) Niveau. Diese wurden in beiden spektralen Fenstern mit je einem Faktor (O-H Streckbereich: 0.964, Ferninfrarotbereich: 0.882) skaliert, der sich aus den experimentellen und theoretischen Wellenzahlen des intensiven Tetramerübergangs ergibt. So kann die hohe Anharmonizität der Librationsübergänge für den Vergleich zwischen harmonischer Theorie und Experiment ausgeglichen werden. Im Ferninfrarotbereich sind die Clusterschwingungen, bei denen eine Torsion der $\mathrm{O}-\mathrm{H}$ Gruppen vorliegt, die einzigen mit signifikanter Intensität, sodass Schwingungen mit geringerer Anharmonizität wie Knickschwingungen der Schweratome für die Zuordnung der Spektren nicht relevant sind. Eine Ausnahme wäre, wenn 
sie durch Resonanz mit den Torsionsschwingungen an Intensität gewinnen. Die vorhergesagten Intensitäten im Ferninfrarotbereich ergeben sich aus der Skalierung der berechneten Intensitäten für jede Clustergröße auf die intensiven Clustersignale im O-H Streckschwingungsbereich. ${ }^{37,223}$ Aus der Auswertung der $\mathrm{O}-\mathrm{H}$ Streckschwingungsspektren ergibt sich auch eine Abschätzung der relativen Häufigkeitsverhältnisse der Cluster im gemessenen Bereich der Überschallexpansionen. Für das untere Spektrum in Abbildung 5.7 ist das Verhältnis von Dimer:Trimer:Tetramer 1:0.3:0.4. Absolut sind im Vergleich zur ausgewerteten Jetexpansion von Methanol in Kapitel 5.2.1 etwa $20 \%$ mehr Dimere vorhanden, während etwa $50 \%$ weniger Trimere von tertButylalkohol vorliegen. Dies erklärt zum Teil die bessere Detektierbarkeit der Dimersignale im Ferninfrarotbereich.

Für den Dimerdonor werden zwei Librationsübergänge vorhergesagt, wobei in der harmonischen Vorhersage derjenige bei höherer Wellenzahl aus der Mischung mit der symmetrischen Streckschwingung der Schweratome des Dimerakzeptors entsteht. Da in Wirklichkeit diese beiden Übergänge weiter auseinanderliegen als in der harmonischen Rechnung, ist es fraglich, ob diese Bande auch in der Realität diese relativ hohe Intensität besitzt. Wahrscheinlicher ist, dass sie eine ähnlich geringe Intensität wie die entsprechende Schwingung des Dimerdonors aufweist. Die Zuordnung des intensiveren Librationsübergangs zur Dimerbande bei $614 \mathrm{~cm}^{-1} \mathrm{er}-$ gibt jedoch Sinn. Für den Dimerakzeptor werden zwei Torsionsübergänge vorhergesagt, die sich ähnlich zum Monomer aus unterschiedlicher Kombination mit der Methylrotation und hier zusätzlich mit einer kleinamplitudigen Donor $\mathrm{O}-\mathrm{H}$ Libration ergeben. Eine Zuordnung zu den zwei Clusterbanden im niedrigen Ferninfrarotbereich liegt nahe, da die vorhergesagten Intensitäten etwa übereinstimmen und die anderen Clustergrößen hier keine intensiven Banden zeigen sollten. Zudem passt in etwa die vorhergesagte Aufspaltung zwischen diesen beiden Banden.

Während für das Tetramer keine Banden mit signifikanter Intensität im Ferninfrarotbereich vorhergesagt werden, sollte das Trimer zwei unterschiedlich intensive Librationsbanden zeigen. Die intensivere Librationsschwingung kann damit der Trimerbande bei $565 \mathrm{~cm}^{-1}$ zugeordnet werden. So ergibt sich für die weniger intensive Librationsschwingung bei höherer Wellenzahl die Zuordnung zu dem Signal bei 632 bis $642 \mathrm{~cm}^{-1}$ im Spektrum entsprechend der vorhergesagten Intensität und Aufspaltung zwischen diesen beiden Übergängen. In der Literatur findet sich eine abweichende Zuordnung der tert-Butylalkohol-Trimersignale. ${ }^{13}$ Der Bande bei $614 \mathrm{~cm}^{-1}$ wurde nicht das Dimer, sondern das Trimer zugeordnet. Jedoch passt diese Zuordnung weder zur vorhergesagten Intensität der Bande, noch zur vorhergesagten Position gegenüber der intensiveren Trimerbande. Vor allem widerspricht sie der hier getroffenen experimentellen Zuordnung auf Grundlage der Konzentrations- und Druckabhängigkeit der Clustersignale. Abweichend zu Referenz 13 ergibt sich somit eine Aufspaltung zwischen den beiden intensiveren Librationsübergängen des Trimers von 67 bis $77 \mathrm{~cm}^{-1}$. Diese Aufspaltung ist höher als im Methanoltrimer $\left(59 \mathrm{~cm}^{-1}\right)$, was vor dem Hintergrund stärkerer Wasserstoffbrücken zu erwarten ist. Auch in den harmonischen Vorhersagen auf B3LYP-D3/6-311+G(2d,p)-Niveau wird eine deutliche Zunahme dieser Aufspaltung zwischen den Trimerlibrationen von Methanol zu tert-Butylalkohol von etwa $60 \%$ vorhergesagt. Durch die abweichenden Zuordnung in Referenz 13 ergab sich dagegen eine kleinere Aufspaltung für tert-Butylalkoholtrimer von $49 \mathrm{~cm}^{-1}$ als für Methanoltrimer.

In Spektren bei besonders hoher tert-Butylalkoholkonzentration von $0.6 \%$ und mehr erscheinen zwei weitere Banden bei $422 \mathrm{~cm}^{-1}$ und $353 \mathrm{~cm}^{-1}$, die wahrscheinlich Clustern größer als 
dem Tetramer zuzuordnen sind.

\subsection{2. tert-Butylalkohol-OD}

Durch die Deuterierung der Hydroxygruppe erfährt ihre Torsionsschwingung eine deutliche Rotverschiebung. Trotzdem sind in den Neon-Matrixspektren von tert-Butylalkohol-OD ${ }^{219}$ in Abbildung 5.8 noch schwache Übergänge der O-D Torsion zu erkennen. In den Jetspektren ist im niedrigen Wellenzahlbereich das Signal-zu-Rauschen Verhältnis schlecht, jedoch lässt sich eine Bande bei $222 \mathrm{~cm}^{-1}$ reproduzieren, die entweder dem Monomer oder dem Dimerakzeptor von tert-Butylalkohol zuzuordnen ist.

Die intensiven Knickschwingungen der Schweratome sind in den Jetspektren bei $340 \mathrm{~cm}^{-1}$ und im Bereich von 454 bis $479 \mathrm{~cm}^{-1}$ zu finden. Die Monomerübergänge im Ferninfrarotbereich sind in Tabelle 5.5 zusammengefasst.

Tabelle 5.5.: Bandenpositionen in $\mathrm{cm}^{-1}$ von tert-Butylalkohol-OD im Bereich von 200 bis $670 \mathrm{~cm}^{-1}$ : Experimentelle Ergebnisse in verschiedenen Umgebungen und harmonische Vorhersagen auf B3LYP-D3/6-311+G(2d,p) Niveau, für die auch Intensitäten in $\frac{\mathrm{km}}{\mathrm{mol}}$ in Klammern gegeben sind. Die Zuordnung der Normalmoden beruht auf der quantenchemischen Vorhersage.

\begin{tabular}{lcccc}
\hline & B3LYP-D3 & He Jetexpansion & Ne-Matrix ${ }^{219}$ & Ar-Matrix ${ }^{222}$ \\
\hline O-D-Torsion & $198(42)$ & & & \\
& $222(15)$ & 222 & 226 & \\
Methyl-Torsion & $276(0.9)$ & & & \\
& $281(0.1)$ & 340 & 340 & 344.4 \\
C-C-C Knick & $345(1.9)$ & & 344 & \\
& $349(4.3)$ & $454-479$ & 417 & 418.3 \\
symm. C-C-O Knick & $425(0.3)$ & & 446 & 442.5 \\
asymm. C-C-O Knick & $458(9)$ & & 463 & \\
\hline
\end{tabular}

Im O-deuterierten tert-Butylalkohol liegen die Wellenzahlen der O-D Torsionsübergänge der Cluster bei deutlich niedrigeren Wellenzahlen. Dadurch lässt sich auch die intensive Librationsbande des Tetramers beobachten. Die Zuordnung bei $571 \mathrm{~cm}^{-1}$ kann auf Grund der guten Separierung dieser Bande von anderen Signalen anhand des Wachstumsverhaltens getroffen werden. Außerdem entspricht die Lage der Bande einem zu erwartenden Isotopenverhältnis von 1.36 sehr ähnlich dem Verhältnis, das auch für das Methanol Tetramer erhalten wurde (siehe Tabelle 5.3). Deshalb wurde diese Bande verwendet um die in harmonischen Schwingungsrechnungen auf B3LYP-D3/6-311+G(2d,p) Niveau erhaltenen Wellenzahlen für den den direkten Vergleich mit den Spektren in Abbildung 5.8 zu skalieren (Skalierungsfaktor: 0.8828).

Die Librationsbanden des Dimers und Trimers liegen im gleichen Wellenzahlbereich wie die intensiven $\mathrm{C}-\mathrm{C}-\mathrm{O}$ Knickschwingungen des Monomers und überlappen mit deren Signalen teilweise. Dies erschwert die Zuordnung dieser Banden. Zwei Clusterbanden lassen sich jedoch bei 


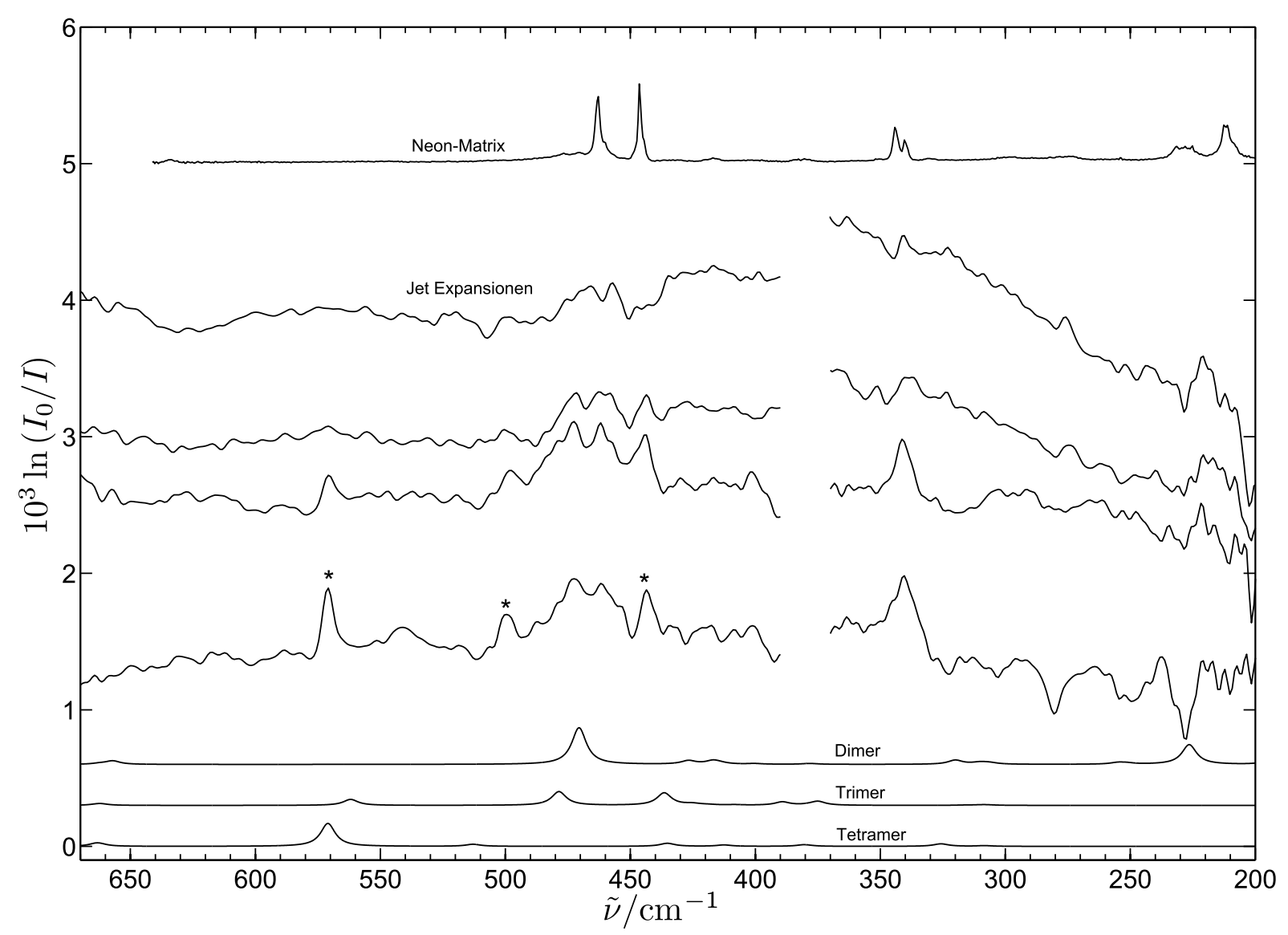

Abbildung 5.8.: Vergleich der Spektren von tert-Butylalkohol-OD in Jetexpansionen und in einer Neon Matrix mit quantenchemischen Frequenzvorhersagen zu tertButylalkohol-OD Clustern im Ferninfrarotbereich. Die Spektren in der Neonmatrix ${ }^{219}$ wurden herunterskaliert, sodass die Intensitäten der Übergänge etwa mit den Monomerbanden in den Jetspektren übereinstimmen. In den experimentellen Jetspektren steigt die Clusterkonzentration von oben nach unten: $0.06 \%$ tert-Butylalkohol in 0.4 bar Helium (oben, 200 Pulse), 0.09\% tert-Butylalkohol in 0.4 bar Helium (zweites von oben, 200 Pulse), 0.09\% tert-Butylalkohol in 0.6 bar Helium (drittes von oben, 200 Pulse), $0.16 \%$ tert-Butylalkohol in 0.7 bar Helium (unten, 200 Pulse). Die Wellenzahlen der harmonischen Schwingungsrechnungen (B3LYP-D3/6-311+G(2d,p)) wurden mit dem Faktor 0.8828 skaliert, der die intensiven Tetramerbanden aus Experiment und Rechnung in Übereinstimmung bringt. Die Clusterkonzentrationen wurden mit Hilfe von tertButylalkohol-OH Jetspektren unter identischen Bedingungen im $\mathrm{O}-\mathrm{H}$ Streckschwingungsbereich abgeschätzt und die vorhergesagten Intensitäten für jede Clustergröße entsprechend skaliert. Die experimentell gefundenen Clusterbanden sind im Ferninfrarotbereich mit Sternchen markiert. 


\section{5. $\mathrm{O}-\mathrm{H}$ Torsionen}

444 und $499 \mathrm{~cm}^{-1}$ eindeutig identifizieren. Die Abhängigkeit ihrer Intensität von Stagnationsdruck und Substanzkonzentration deutet für die Bande bei $499 \mathrm{~cm}^{-1}$ auf einen Trimerursprung, während sich die Größenzuordnung der Bande $444 \mathrm{~cm}^{-1}$ schwieriger darstellt, tendenziell aber eher dem Dimer zugeordnet werden könnte. Dies entspricht nicht einer Bandenverteilung der Dimer- und Trimerlibrationen, wie sie analog zu den den tert-Butylalkohol-OH Spektren erwartet werden könnte oder wie sie sich in der harmonischen Vorhersage ergibt. Der Grund dafür könnte sein, dass die Librationsübergänge des Dimers und Trimers von tert-Butylalkohol-OD sehr nah an den weniger intensiven $\mathrm{C}-\mathrm{C}-\mathrm{O}$ Knickschwingungsübergängen dieser Cluster liegen. Dadurch könnten durch Kopplung diese Schwingungen miteinander mischen und/oder sich die Bandenpositionen signifikant ändern. In den harmonischen Frequenzrechnungen, liegen die Bandenpositionen dieser Schwingungen zu weit auseinander, als dass sich dort ein deutlicher Effekt ergeben könnte. Somit muss für tert-Butylalkohol nicht zwingend das gleiche Bandenmuster für Dimere und Trimere der O-D Spezies wie für die $\mathrm{O}-\mathrm{H}$ Spezies erwartet werden. Im Falle des Methanoldimers und -trimers ergeben sich dagegen sehr ähnliche Spektren, da die Librationsübergänge der Isotopologen gut separiert von anderen intramolekularen Schwingungen liegen (Vgl. Abschnitt 5.2.1 \& 5.2.2).

Laut der harmonischen Rechnungen werden für das Trimer insgesamt drei Librationsübergänge erwartet. Die Bande bei höchster Wellenzahl sollte in der Nähe der Tetramerlibrationsbande liegen, jedoch kann kein reproduzierbares Signal gefunden werden, das diesem wenig intensiven Librationsübergang zugeordnet werden könnte. Das zugeordnete Trimersignal im Jetspektrum bei $499 \mathrm{~cm}^{-1}$ entspricht vermutlich der Trimerlibration in mittlerer Position, sodass die Librationsbande mit der niedrigsten Wellenzahl wahrscheinlich von den intensiven Monomersignalen im Bereich von 454 bis $479 \mathrm{~cm}^{-1}$ überlagert wird. Bei etwa $473 \mathrm{~cm}^{-1}$ auf der breiten Monomerbande scheint tatsächlich etwas mehr Intensität mit höherer Clusterkonzentration aufzutreten - ein Hinweis dass in der Jetexpansion Monomer- und Clustersignale überlagern. In NeonMatrizen wird die Libration des Dimerdonors von tert-Butylalkohol-OD bei $476 \mathrm{~cm}^{-1}$ beobachtet. Auf Grund der guten Übereinstimmung zwischen Position der Dimersignale in Jetspektren und Neonmatrixspektren im Falle des undeuterierten tert-Butylalkohol spricht dieser Matrixbefund eher für die Zuordnung der Dimerdonorlibration zum Signal bei $473 \mathrm{~cm}^{-1}$ in den Jetspektren. Andererseits kann auch im Matrix-isolierten Dimer die Libration des Dimerdonors mit den oben erwähnten $\mathrm{C}-\mathrm{C}-\mathrm{O}$ Knickschwingungen wechselwirken, was zu deutlichen Verschiebungen gegenüber der ungestörten Position der Librationsbande führen kann. Insofern kann die Übereinstimmung zwischen der Bandenposition in Neonmatrizen mit dem Signal bei $473 \mathrm{~cm}^{-1}$ in den Jetspektren auch irreführend sein.

Insgesamt kann die experimentelle Zuordnung der intensiven Tetramerlibration als zuverlässig eingestuft werden. Die Zuordnung der mittleren Trimerbande bei $499 \mathrm{~cm}^{-1}$ ist ebenfalls recht solide. Weniger sicher ist jedoch die Zuordnung des Signals bei $444 \mathrm{~cm}^{-1}$ zum Dimer. Es könnte hier auch das Trimer zugeordnet werden, sodass sich für tert-Butylalkohol-OD eine größere Aufspaltung für die intensiven Trimerlibrationen von $55 \mathrm{~cm}^{-1}$ gegenüber Methanol-OD mit $46 \mathrm{~cm}^{-1}$ ergibt, wie sie auch im Falle der undeuterierten Systeme beobachtet wird. Außerdem würden dann für beide tert-Butylalkohol Trimerlibrationen identische Isotopenverhältnisse der $\mathrm{O}-\mathrm{H}$ zu O-D Wellenzahlen von etwa 1.27 resultieren (s. Tabelle 5.6), diese wären jedoch deutlich geringer als im Falle der Methanoltrimere (s. Tabelle 5.3). 


\subsubsection{Zusammenfassung}

Tabelle 5.6.: Experimentelle Wellenzahlen der intensivsten $\mathrm{O}-\mathrm{H}$ bzw. $\mathrm{O}-\mathrm{D}$ Torsionsübergänge von verschiedenen tert-Butylalkohol Clustergrößen. Zusätzlich ist das Verhältnis der Wellenzahlen $\frac{\tilde{\nu}_{\mathrm{OH}}}{\tilde{\nu}_{\mathrm{OD}}}$ zwischen den Schwingungen der undeuterierten und Odeuterierten Spezies gegeben.

\begin{tabular}{lccc}
\hline & $\tilde{\nu} / \mathrm{cm}^{-1}$ & $\tilde{\nu} / \mathrm{cm}^{-1}$ & $\frac{\tilde{\nu}_{\mathrm{OH}}}{\tilde{\nu}_{\mathrm{OD}}}$ \\
\hline Torsion O-H & Torsion O-D & \\
& {$[261]$} & & $1.38 / 1.30$ \\
Timer & 290 & 444 oder $\approx 473$ & $1.19 / 1.27$ \\
\multirow{4}{*}{ Tetramer } & 614 & $\approx 473$ oder 444 & $1.27-1.29$ \\
& 565 & 499 & 1.36 \\
\hline
\end{tabular}

${ }^{a}$ Referenz 13.

Im Falle von undeuteriertem tert-Butylalkohol gelingt die experimentelle Zuordnung der Clusterbanden mit Hilfe quantenchemischer Rechnungen recht gut. Die Bande der Dimerdonorlibration liegt $57 \mathrm{~cm}^{-1}$ höher als für den Dimerdonor von Methanol (Neon-Matrix Spektrum). Dies spricht für eine stärkere Wasserstoffbrücke im tert-Butylalkoholdimer als im Methanoldimer. Jedoch übertrifft diese höhere Wellenzahl die harmonische Vorhersage auf B3LYP-D3/6$311+\mathrm{G}(2 \mathrm{~d}, \mathrm{p})$ Niveau, dass die Dimerlibration von tert-Butylalkohol $19 \mathrm{~cm}^{-1}$ höher liegt als die des Methanoldimers. Das könnte ein Hinweis auf eine geringere Anharmonizität der Dimerdonorlibration von tert-Butylalkohol sein. Auch die intensiven Librationsbanden der Trimere und Tetramere von tert-Butylalkohol liegen relativ zu den entsprechenden Übergängen der Methanolcluster bei höheren Wellenzahlen als die harmonischen Rechnungen vorhersagen, was ebenfalls auf eine geringere Anharmonizität der Librationen in den tert-Butylalkoholclustern hindeutet. Die Zuordnung der experimentellen Librationsübergänge der undeuterierten und Odeuterierten Cluster von tert-Butylalkohol ist in Tabelle 5.6 zusammengefasst.

\subsection{Ethanol}

Die zwei chiralen Minima des Torsionspotentials von Ethanol, die gauche Konformationen entsprechen, führen zu einer Tunnelaufspaltung der Schwingungszustände von gauche-Ethanol (Vgl. Abbildung 4.12 in Kapitel 4.2.3), die entsprechend ihrer Symmetrie mit + oder - gekennzeichnet werden können. ${ }^{59,182,224}$ Im Schwingungsgrundzustand ist die Tunnelaufspaltung klein, der gauche- $(\mathrm{g}-)$ liegt $3.3 \mathrm{~cm}^{-1}$ über dem gauche $+(\mathrm{g}+)$ Zustand. ${ }^{178}$ Das trans-Isomer $(\mathrm{t})$ ist energetisch günstiger und wird deshalb in Jetexpansionen bevorzugt gebildet.

Die energetisch niedrigsten Übergänge liegen außerhalb des spektroskopischen Fensters des Filet-Jet und entsprechen der Anregung innerhalb von gauche-Ethanol in den angeregten $\mathrm{g}+\mathrm{Zu}-$ stand (Vgl. Tabelle 5.7). Dabei unterscheidet sich die Wellenzahl aus IR-Absorptionsmessungen ${ }^{224}$ 
Tabelle 5.7.: Bandenpositionen der Übergänge von Ethanolmonomer (in $\mathrm{cm}^{-1}$ ) im Ferninfrarotbereich: Experimentelle Ergebnisse aus FTIR- und Ramanmessungen von Ethanol in He-Jetexpansionen und IR-Absorptionsmessungen der Gasphase. Außerdem sind für die Torsionsübergänge die vorhergesagte Wellenzahlen aus Analyse eines theoretischen $\mathrm{O}-\mathrm{H}$ Torsionspotentials gegeben. Die Zustände sind entsprechend der Nomenklatur in Referenz 182 und 59 angegeben. Wellenzahlen von Übergängen deren Zuordnung unsicher ist sind in eckigen Klammern geschrieben.

\begin{tabular}{lcccc}
\hline & $\begin{array}{c}\text { FTIR- } \\
\text { Jetspektrum }\end{array}$ & $\begin{array}{c}\text { IR- } \\
\text { Gasphase }^{224}\end{array}$ & $\begin{array}{c}\text { Raman- } \\
\text { Jetspektrum }^{59,182}\end{array}$ & Vorhersage $^{225}$ \\
\hline $\begin{array}{l}\mathrm{O}-\mathrm{H}-\text { Torsion } \\
\mathrm{g}_{0}^{-} \rightarrow \mathrm{g}_{1}^{+}\end{array}$ & & 195.8 & & 204.5 \\
$\mathrm{~g}_{0}^{+} \rightarrow \mathrm{g}_{1}^{+}$ & & & 199 & 207.5 \\
$\mathrm{t}_{0} \rightarrow \mathrm{t}_{1}$ & 203 & 202.6 & 202 & 205.5 \\
$\mathrm{~g}_{0}^{-} \rightarrow \mathrm{g}_{1}^{-}$ & & & {$[240]$} & 249.8 \\
$\mathrm{~g}_{0}^{+} \rightarrow \mathrm{g}_{1}^{-}$ & 246 & 243.1 & & 252.8 \\
$\mathrm{t}_{0} \rightarrow \mathrm{g}_{1}^{-}$ & {$[288]$} & & {$[283]$} & 278.3 \\
$\mathrm{t}_{0} \rightarrow \mathrm{t}_{2}$ & {$[353]$} & & & 352.8 \\
$\mathrm{C}-\mathrm{C}-\mathrm{O}$ Knick & 418 & $418(\mathrm{t})$ & $417(\mathrm{t})$ & \\
& & $420(\mathrm{~g})$ & $420(\mathrm{~g})$ & \\
\hline
\end{tabular}

um etwa $3 \mathrm{~cm}^{-1}$ von den Ramanwerten, ${ }^{59,182}$ woraus geschlossen wurde, dass für beide Messmethoden komplementäre Auswahlregeln für die Anregung der O-H Torsion vorliegen: ${ }^{182}$ bei Ramananregung sollte die Tunnelsymmetrie der gauche-Zustände erhalten bleiben, während sie sich durch IR-Absorption ändert.

In Abbildung 5.9 sind die Raman- ${ }^{59,182}$ und FTIR Spektren von Ethanol in He-Jetexpansionen gezeigt. Die Wellenzahlen der Monomerübergänge und ihre Zuordnung sind in Tabelle 5.7 mit Literaturwerten zusammengefasst. Im FTIR Jetspektrum zeigt sich eine intensive Bande bei $203 \mathrm{~cm}^{-1}$, die in Übereinstimmung mit IR Gasphasenmessungen ${ }^{224}$ der Torsionsfundamentale von trans-Ethanol zugeordnet werden kann. Bei $246 \mathrm{~cm}^{-1}$ befindet sich ein weiterer Übergang der wahrscheinlich der $\mathrm{g}+\rightarrow \mathrm{g}-$ Anregung entspricht. In der warmen Gasphase wurde der QZweig dieser Bande bei $243.1 \mathrm{~cm}^{-1}$ gefunden, ${ }^{224}$ was durch thermische Effekte hervorgerufen werden könnte. In Raman Jetmessungen wurde bei $243 \mathrm{~cm}^{-1}$ die Methyltorsion zugeordnet dies jedoch auf Grundlage der warmen Gasphasenmessung in Referenz 224 und der Erwartung, dass eine Aufspaltung von $3.3 \mathrm{~cm}^{-1}$ zwischen Raman- und IR-Messung vorliegen sollte, die auch $\mathrm{zu}$ einer tendenziellen Zuordnung des $\mathrm{g}-\rightarrow \mathrm{g}-$ Übergangs bei $240 \mathrm{~cm}^{-1}$ führte. Die im FTIR Jetspektrum beobachtete Position von $246 \mathrm{~cm}^{-1}$ für $\mathrm{g}+\rightarrow \mathrm{g}-$ spricht jedoch für eine Zuordnung der Bande im Raman Jetspektrum bei $243 \mathrm{~cm}^{-1}$ zum g- $\rightarrow \mathrm{g}-$ Torsionsübergang, in Einklang mit der Grundzustandsaufspaltung ${ }^{178}$ und den komplementären Auswahlregeln. Somit wäre auch die Energie des Torsions-angeregten $\mathrm{g}-$ Zustands in den Niveauschemata von Referenz 182 und 59 von $282.6 \mathrm{~cm}^{-1}$ auf $285 \mathrm{~cm}^{-1}$ zu korrigieren. Damit läge bei angeregter Torsionsschwingung eine Tunnelaufspaltung von $46 \mathrm{~cm}^{-1}$ zwischen den gauche Zuständen vor.

Im FTIR Jetspektrum findet sich bei $288 \mathrm{~cm}^{-1}$ eine weitere Bande. In diesem Bereich wur- 


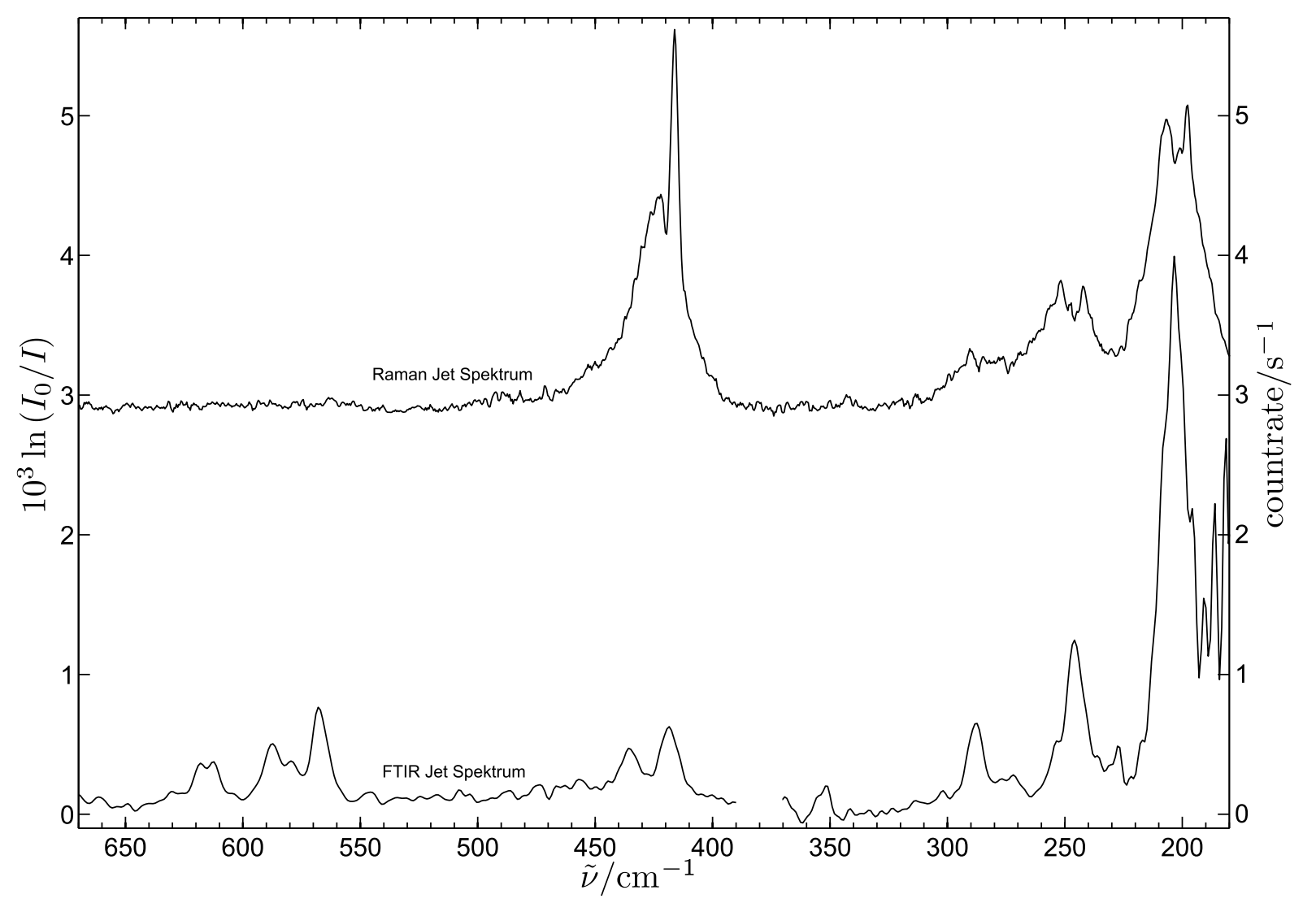

Abbildung 5.9.: Vergleich der Spektren von Ethanol in Jetexpansionen des Filet-Jet (FTIR) und des Curry-Jet (Raman). Das FTIR-Spektrum wurde von $0.14 \%$ Ethanol in 0.7 bar Helium aufgenommen (390 Pulse). Das Ramanspektrum wurde von Tobias Wassermann gemessen, bereits veröffentlicht ${ }^{59,182}$ und zeigt Expansionen von $0.3 \%$ Ethanol in 0.8 bar Helium bei einem Düsenabstand von $1 \mathrm{~mm}$.

den im Ramanspektrum der Übergang $\mathrm{t} \rightarrow \mathrm{g}$ - unter Änderung der Konformation vermutet, jedoch passt die Position der Bande im FTIR Jetspektrum nicht genau zur erwarteten Position von $285 \mathrm{~cm}^{-1}$. Alternativ könnte die Bande bei $288 \mathrm{~cm}^{-1}$ auch Clustern zuzuordnen sein, was sich durch Variation von Ethanolkonzentration und Stagnationsdruck herausfinden lassen sollte. Dabei wäre eine Zuordnung zu einem Dimerakzeptor wahrscheinlich, wobei aber eigentlich mehrere Signale von Dimerakzeptoren zu erwarten sind, da Ethanol in He-Jetexpansionen in verschiedenen Dimergeometrien vorkommt. Da in diesen entweder die gauche- oder trans-Konformation für den Dimerakzeptor ${ }^{59}$ vorliegen kann, ist zu erwarten, dass sich die Positionen der Torsionsübergänge der Dimerakzeptoren auch deutlich unterscheiden. Ein weiteres Signal bei $353 \mathrm{~cm}^{-1}$ könnte ebenfalls dem Monomer oder Clustern zuzuordnen sein. Für die Zuordnung zum Monomer spricht, dass bei genau dieser Position der Torsionsoberton von trans-Ethanol vorhergesagt wird. ${ }^{225}$ Andererseits wurden für tert-Butylalkohol-OH an gleicher Position auch Signale größerer Cluster beobachtet (Vgl. Kapitel 5.3.1). Durch weitere Untersuchung der Druck- und Kon- 


\section{5. $\mathrm{O}-\mathrm{H}$ Torsionen}

zentrationsabhängigkeit dieser Bande sollte jedoch die Zuordnung zu einer der Möglichkeiten einfach realisierbar sein.

Bei $418 \mathrm{~cm}^{-1}$ ist im FTIR Jetspektrum vornehmlich die Anregung der C-C $-\mathrm{O}$ Knickschwingung des trans-Monomers zu beobachten. ${ }^{59,182}$ Das entsprechende Signal des weniger populierten gauche-Ethanols sollte davon überdeckt werden, da es bei ähnlicher Position liegt (Vgl. Tabelle 5.7). Daneben zeigt sich im Absorptionsspektrum eine Bande bei $436 \mathrm{~cm}^{-1}$ des jetgekühlten Ethanols, welches im Ramanspektrum bei 419 bis $450 \mathrm{~cm}^{-1}$ eine breite und unstrukturierte Clusterbande aufweist. Bei $436 \mathrm{~cm}^{-1}$ wurde bisher keine Monomerbande beobachtet, jedoch wäre eine Zuordnung zu den Torsionsobertönen der gauche-Ethanole möglich, die bei $445 \mathrm{~cm}^{-1}$ vorhergesagt werden. ${ }^{225}$ Eine Zuordnung zu Clustern ist jedoch ebenfalls in Betracht zu ziehen. Dabei spricht die relativ hohe Intensität und die Schmalheit der Bande eher gegen eine Überlagerung der $\mathrm{C}-\mathrm{C}-\mathrm{O}$ Knickschwingungen verschiedener Clustergrößen an dieser Position. Wahrscheinlicher wäre ein Übergang einer einzelnen Clusterspezies.

Tabelle 5.8.: Berechnete Wellenzahlen der $\mathrm{O}-\mathrm{H}$ Torsionen der Dimerakzeptoren und -donoren in harmonischen und anharmonischen Schwingungsrechnungen auf B3LYP-D3/6$311+\mathrm{G}(2 \mathrm{~d}, \mathrm{p})$ Niveau. Die Nomenklatur der Dimere entspricht den Referenzen 182 und 59 .

\begin{tabular}{lcccc}
\hline & \multicolumn{2}{c}{ Dimer Akzeptor } & \multicolumn{2}{c}{ Dimer Donor } \\
& harm. & anharm. & harm. & anharm. \\
\hline $\mathrm{g}-\mathrm{g}-$ & $337(91)$ & $304(98)$ & $702(95)$ & $611(90)$ \\
$\mathrm{g}-\mathrm{t}$ & $337(102)$ & $336(84)$ & $700(107)$ & $579(98)$ \\
$\mathrm{g}+\mathrm{t}$ & $330(98)$ & $280(77)$ & $709(103)$ & $599(94)$ \\
\hline
\end{tabular}

Durch den direkten Vergleich mit dem Ramanspektrum lassen sich jedoch im Bereich der $\mathrm{O}-\mathrm{H}$ Libration eindeutig Clusterbanden identifizieren. Das Ramanspektrum zeigt in diesem Bereich keine Signale und es liegen in diesem Bereich keine Fundamentalübergänge des Monomers. ${ }^{226}$ Die intensivste Librationsbande liegt bei $567 \mathrm{~cm}^{-1}$, eine weitere bei $587 \mathrm{~cm}^{-1}$ mit einer Schulter bei $579 \mathrm{~cm}^{-1}$ und ein weiteres Signal bei $615 \mathrm{~cm}^{-1}$. Möglicherweise kann es durch umfassende Untersuchung der Ethanolkonzentrations- und vor allem Druckabhängigkeit der Intensität dieser Banden gelingen, ihre Clustergrößen zuzuordnen. Zusätzlich könnten durch Verwendung von Neon als Trägergas oder durch Argonbeimischung die Signale der stabilsten Dimere identifiziert werden. ${ }^{59,181}$ In Tabelle 5.8 sind die berechneten Wellenzahlen der O-H Torsion für das stabilste Dimer (gauche, gauche) und die beiden möglichen Dimerstrukturen des zweitstabilsten Dimers (gauche-Donor, trans-Akzeptor) gegeben. ${ }^{59}$ Sie zeigen, dass sich im Bereich der Donorlibration die Wellenzahlen der unterschiedlichen Dimerstrukturen unterscheiden, was vor allem an den anharmonischen Korrekturen in den Rechnungen liegt. Somit eröffnen die Librationsübergänge der Dimere eventuell die Möglichkeit die Struktur des zweitstabilsten Dimers $(\mathrm{g}-\mathrm{t} \text { oder } \mathrm{g}+\mathrm{t})^{59}$ spektroskopisch mit Hilfe anharmonischer Vorhersagen zu identifizieren. In Argonmatrizen wird ein Dimersignal bei $651 \mathrm{~cm}^{-1}$ beobachtet, ${ }^{227}$ das vermutlich der Donorlibration zugeordnet werden kann. Es erscheint gegenüber den hier gelisteten Librationssignalen in He-Expansionen deutlich blauverschoben. 


\subsection{Trifluorethanol}

\subsection{Trifluorethanol}

\subsubsection{Trifluorethanol-OH}

Trifluorethanol liegt in der Gasphase fast (oder sogar) ausschließlich im gauche-Zustand vor, ${ }^{188,229,230}$ was an einer verhältnismäßig kleinen Barriere für die Umwandlung des energetisch ungünstigeren trans-Konformers in die stabileren O-H Rotamere liegt. ${ }^{188,231-233}$ Zwei enantiomere gaucheMinima des $\mathrm{O}-\mathrm{H}$ Torsionspotentials führen zu einer geringen Tunnelaufspaltung zwischen den zwei gauche-Zuständen von $0.2 \mathrm{~cm}^{-1}$ im Schwingungsgrundzustand. ${ }^{230}$ In Überschallexpansionen wurde bisher ebenfalls nur das gauche-Monomer beobachtet. ${ }^{188}$ In Abbildung 5.10 sind die Raman- (gemessen von Katharina Otto ${ }^{228}$ ) und FTIR-Spektren von Trifluorethanol in Überschallexpansionen von Helium im Ferninfrarotbereich gezeigt. Darin lassen sich die markanten Monomerbanden, die bereits für die warme Gasphase in der Literatur beschrieben wurden, ${ }^{224}$ leicht erkennen. Sie sind zusammen mit schwächeren Signalen aus den Spektren in Tabelle 5.9 zusammengefasst. Die IR-intensiven Monomerbanden zeigen in den Filet-Jetspektren jedoch ein unterschiedliches Intensitätsverhalten. Während die $\mathrm{O}-\mathrm{H}$ Torsionsbande bei $283 \mathrm{~cm}^{-1}$ und die Schaukelschwingung der $\mathrm{CF}_{3}$ Gruppe bei $364 \mathrm{~cm}^{-1}$ nur eine leichte Intensitätszunahme durch Erhöhung der absoluten Trifluorethanolkonzentration zeigen, steigen die Intensitäten der symmetrischen $\mathrm{F}-\mathrm{C}-\mathrm{C}-\mathrm{O}$ Knickschwingung bei $418 \mathrm{~cm}^{-1}$ und der Deformationsschwingungen der $\mathrm{CF}_{3}$ Gruppe bei $548 \mathrm{~cm}^{-1}$ und $665 \mathrm{~cm}^{-1}$ vom oberen zum unteren FTIR-Spektrum in Abbildung 5.10 deutlich stärker an. Es ist unklar woran dieses unterschiedliche Verhalten liegt. Möglich ist, dass die intensive Torsionsbande Intensitäts-senkende Sättigungseffekte zeigt und die Intensität der Schaukelschwingung durch die Nähe des Übergangs zur Transmissionslücke des Strahlteilers beeinflusst wird - dort zeigen sich bei den Spektren höherer Konzentration negative Einbrüche der Basislinie. Aber auch die Intensität der Deformationsschwingung bei $665 \mathrm{~cm}^{-1}$ kann durch ihre Nähe zur Grenze des Transmissionsbereichs des Strahlteilers beeinflusst sein, da hier bei den Spektren mit höherer Konzentration die Basislinie zu hohen Intensitäten ausreißt.

Für die $\mathrm{O}-\mathrm{H}$ Torsion wurde zuvor eine beobachtbare Tunnelaufspaltung der Fundamentalübergänge von $9 \mathrm{~cm}^{-1}$ beschrieben. ${ }^{224}$ Dies kann anhand der Jetspektren widerlegt werden, da hier keine Aufspaltung des Torsionsübergang von dieser Größe beobachtet werden kann. Theoretisch wird eine Tunnelaufspaltung des angeregten $\mathrm{O}-\mathrm{H}$ Torsionszustands von $3.2 \mathrm{~cm}^{-1}$ vorhergesagt. ${ }^{231}$ Da dies unterhalb der experimentellen Auflösung der FTIR-Spektren liegt und die Ramanbande der $\mathrm{O}-\mathrm{H}$ Torsion keine Q-Zweige zeigt, kann eine so geringe Aufspaltung hier nicht überprüft werden.

Im Ramanjetspektrum zeigen sich bei $219 \mathrm{~cm}^{-1}$ und $235 \mathrm{~cm}^{-1}$ zwei schwache Signale, letzteres ist auch in den FTIR-Jetspektren bei $236 \mathrm{~cm}^{-1}$ zu beobachten. Da eine heiße Bande ausgeschlossen werden kann, ${ }^{228}$ handelt es sich dabei höchstwahrscheinlich um den Oberton der $\mathrm{CF}_{3}$ Torsion, deren Fundamentalübergang bei $116 \mathrm{~cm}^{-1}$ liegt, ${ }^{228}$ und die asymmetrische $\mathrm{F}-\mathrm{C}-\mathrm{C}-\mathrm{O}$ Knickschwingung. Die verhältnismäßig hohe Intensität für einen Obertonübergang in beiden Messmethoden lässt sich mit einer Fermiresonanz zwischen den zwei genannten Moden erklären, die auch in der anharmonischen Schwingungsrechnung (B3LYP-D3/6-311+G(2d,p)) vorhergesagt wird. Die beobachtete Aufspaltung von $16 \mathrm{~cm}^{-1}$ zwischen diesen Banden sollte sich auf ungefähr $5 \mathrm{~cm}^{-1}$ im entstörten Fall verringern, wenn man das experimentelle Intensitäts- 

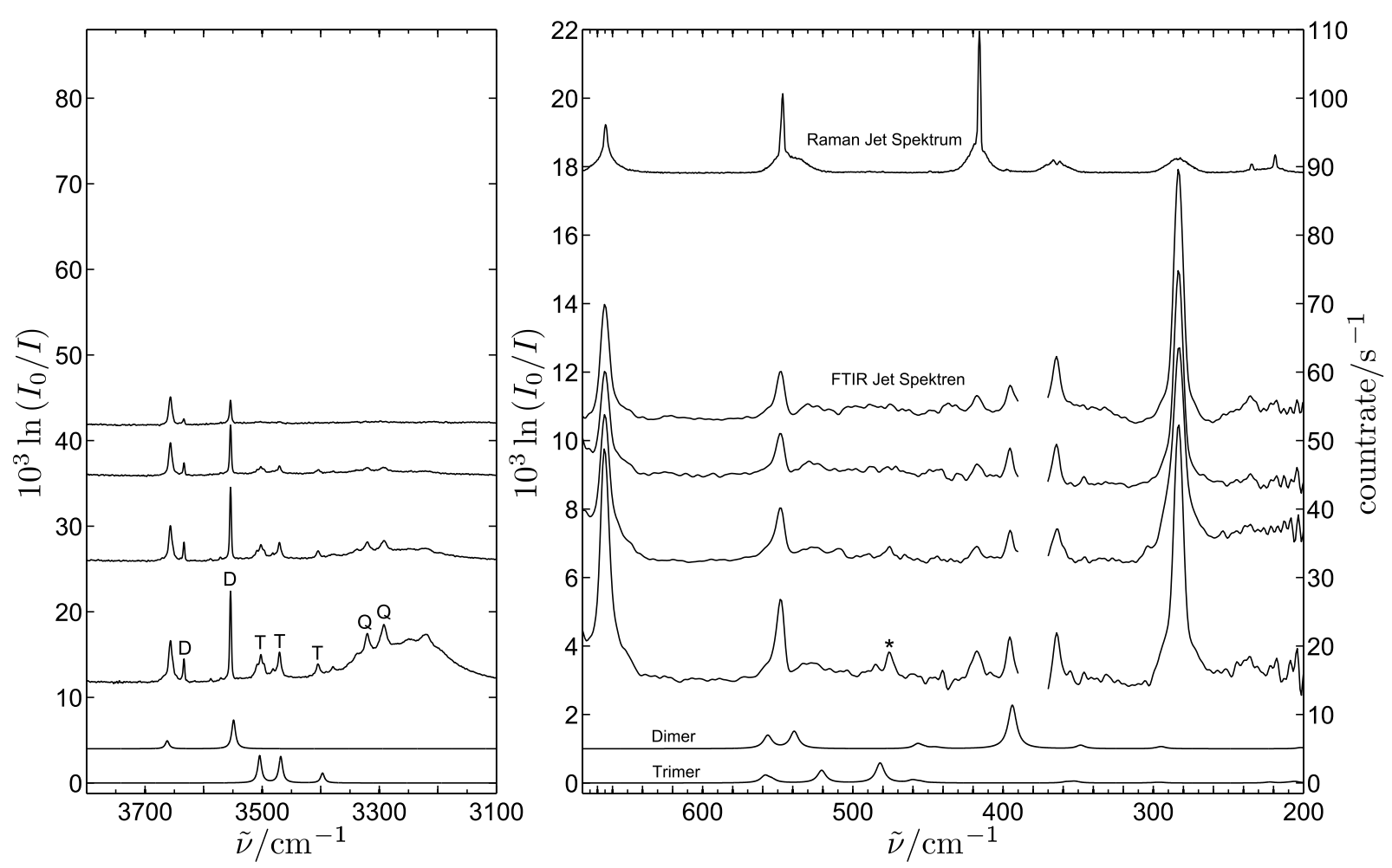

Abbildung 5.10.: Vergleich der Spektren von Trifluorethanol in Jetexpansionen des Filet-Jet (FTIR) und des Curry-Jet (Raman) mit berechneten Clusterspektren. Die FTIR-Spektren zeigen Trifluorethanol unter ähnlichen Bedingungen im $\mathrm{O}-\mathrm{H}$ Streckschwingungs- und Ferninfrarotbereich. Dabei erhöhen sich die absoluten Substanzkonzentrationen und Stagnationsdrücke von oben nach unten: 0.3\% (oben, 0.3 bar, 5 Pulse; FIR: 0.4 bar, 200 Pulse), $0.2 \%$ (zweites von oben, 0.6 bar, 5 Pulse; FIR: 0.6 bar, 100 Pulse), $0.2 \%$ (drittes von oben, 0.9 bar, 5 Pulse; FIR: 0.8 bar, 200 Pulse) und 0.4\% (unten, 0.9 bar, 5 Pulse; FIR: 0.8 bar, 200 Pulse). Das Ramanspektrum wurde von Katharina Otto gemessen ${ }^{228}$ und zeigt Expansionen von $0.1 \%$ Trifluorethanol in 0.7 bar Helium bei einem Düsenabstand von $1 \mathrm{~mm}$. Die Wellenzahlen der harmonischen Schwingungsrechnungen (B3LYP-D3/6-311+G(2d,p)) wurden in beiden Spektralbereichen mit je einem Faktor skaliert, für die der Mittelwert aus den jeweiligen für Methanol und tertButylalkohol verwendeten Skalierungsfaktoren in den Abbildungen 5.3 und 5.7 gewählt wurde. Die berechneten Intensitäten wurden für jede Clustergröße so skaliert, dass die experimentellen Intensitäten im unteren $\mathrm{O}-\mathrm{H}$ Streckschwingungsspektrum mit den berechneten übereinstimmen und sich somit eine Intensitätsvorhersage für das untere Ferninfrarotspektrum ergibt. Die experimentell gefundene Clusterbande im Ferninfrarotbereich ist mit einem Sternchen (*) markiert. 
Tabelle 5.9.: Bandenpositionen der Übergänge von Trifluorethanolmonomer und Clustern im Ferninfrarotbereich in $\mathrm{cm}^{-1}$ : Experimentelle Ergebnisse aus Raman228 und FTIR-Messungen von Trifluorethanol in He-Jetexpansionen und IRAbsorptionsmessungen der Gasphase. ${ }^{224}$ Zusätzlich zu den berechneten Wellenzahlen aus einer anharmonischen Schwingungsrechnung auf B3LYP-D3/6$311+\mathrm{G}(2 \mathrm{~d}, \mathrm{p})-$ Niveau sind die Intensitäten in $\frac{\mathrm{km}}{\mathrm{mol}}$ in runden Klammern gegeben. Die Zuordnungen von Übergängen, die unsicher sind (siehe Text), sind in eckigen Klammern geschrieben. Die Fundamentalübergänge des Monomers sind konsistent mit der Analyse dieser Moden in Ar-Matrizen ${ }^{234,235}$ zugeordnet.

\begin{tabular}{|c|c|c|c|c|}
\hline $\begin{array}{c}\text { FTIR- } \\
\text { Jetspektrum }\end{array}$ & $\begin{array}{c}\text { IR- } \\
\text { Gasphase }^{224}\end{array}$ & $\begin{array}{l}\text { Raman- } \\
\text { Jetspektrum }^{228}\end{array}$ & $\begin{array}{c}\text { B3LYP-D3 } \\
\text { 6-311+G(2d,p) } \\
\text { anharmonisch }\end{array}$ & Zuordnung \\
\hline & 120 & 116 & $113(4)$ & $\mathrm{CF}_{3}$ Torsion \\
\hline & 219 & 218 & $213(6)$ & Fermires.: asymm. $\mathrm{F}-\mathrm{C}-\mathrm{C}-\mathrm{O}$ Knick + \\
\hline 236 & 239 & 235 & $229(0.05)$ & Oberton $\mathrm{CF}_{3}$ Torsion \\
\hline 283 & 281,272 & 283 & $288(115)$ & $\mathrm{O}-\mathrm{H}$ Torsion \\
\hline 346 & & & $333(0.02)$ & {$\left[\mathrm{CF}_{3}\right.$ Tors. + asymm. $\mathrm{F}-\mathrm{C}-\mathrm{C}-\mathrm{O}$ Knick } \\
\hline 364 & 364 & 364 & $355(24)$ & $\mathrm{CF}_{3}$ Schaukelschw. \\
\hline 396 & & & & [Dimerakzeptorlibration] \\
\hline 418 & 424 & 416 & $403(3)$ & symm. $\mathrm{F}-\mathrm{C}-\mathrm{C}-\mathrm{O}$ Knick \\
\hline 440 & & & $437(0.2)$ & [Oberton asymm. $\mathrm{F}-\mathrm{C}-\mathrm{C}-\mathrm{O}$ Knick] \\
\hline 476 & & & & Clusterlibration [Trimer] \\
\hline $485-490$ & & & & [Clusterlibration, Oberton $\mathrm{O}-\mathrm{H}$-Torsion] \\
\hline $521-534$ & & & & [Clusterlibration] \\
\hline 534 & & 534 & $520(0.4)$ & $\mathrm{CF}_{3}$ Deformation \\
\hline 548 & 548 & 547 & $531(8)$ & $\mathrm{CF}_{3}$ Deformation \\
\hline 665 & & 665 & $647(23)$ & $\mathrm{CF}_{3}$ Deformation \\
\hline
\end{tabular}

verhältnis der Signale von etwa 1:2 berücksichtigt und annimmt, dass der Oberton keine eigene Intensität beiträgt. In der anharmonischen Rechnung werden sehr ähnliche Aufspaltungen von $6 \mathrm{~cm}^{-1}$ im entstörten und $16 \mathrm{~cm}^{-1}$ im gestörten Fall vorhergesagt. Somit ergeben sich aus den experimentellen Daten entstörte Bandenpositionen von etwa $222 \mathrm{~cm}^{-1}$ für die asymmetrische $\mathrm{F}-\mathrm{C}-\mathrm{C}-\mathrm{O}$ Knickschwingung und etwa $227 \mathrm{~cm}^{-1}$ für den Oberton der $\mathrm{CF}_{3}$ Torsion $\left(2 \cdot 116 \mathrm{~cm}^{-1}=232 \mathrm{~cm}^{-1}\right)$. Ein weiterer Nachweis der Fermiresonanz ließe sich durch Verbesserung des Signal-zu-Rauschen Verhältnisses der FTIR Spektren erreichen, wenn sich für beide Signale zueinander das gleiche Intensitätsverhältnis im Raman- und Absorptionsspektrum ergibt. In Ar-Matrizen werden ebenfalls beide Signale beobachtet. ${ }^{235}$ Ein weiteres sehr schwaches Signal im FTIR-Jetspektrum bei $346 \mathrm{~cm}^{-1}$ könnte die Kombinationsbande aus $\mathrm{CF}_{3}$ Torsion und der asymmetrischen $\mathrm{F}-\mathrm{C}-\mathrm{C}-\mathrm{O}$ Knickschwingung sein $\left(116 \mathrm{~cm}^{-1}+222 \mathrm{~cm}^{-1}=338 \mathrm{~cm}^{-1}\right)$. Im Ramanspektrum zeigt sich bei $534 \mathrm{~cm}^{-1}$ eine Schulter zur benachbarten Deformationsschwingung der $\mathrm{CF}_{3}$ Gruppe. Anhand der quantenchemischen Vorhersage kann auch für die Schulter eine Deformationsschwingung der $\mathrm{CF}_{3}$ Gruppe zugeordnet werden. In FTIR Spektren findet sich an 


\section{5. $\mathrm{O}-\mathrm{H}$ Torsionen}

dieser Position eine breiter Bereich mit schwacher Absorption von 521 bis $534 \mathrm{~cm}^{-1}$. Weiterhin liegt im FTIR Spektrum ein schwaches Signal bei $440 \mathrm{~cm}^{-1}$ vor, das sich nicht direkt zuordnen lässt. Möglich wäre, dass es sich dabei um den Oberton der asymmetrischen $\mathrm{F}-\mathrm{C}-\mathrm{C}-\mathrm{O}$ Knickschwingung handelt $\left(2 \cdot 222 \mathrm{~cm}^{-1}=444 \mathrm{~cm}^{-1}\right)$.

In He-Expansionen wurde mittels FTIR-Spektroskopie als einzige Dimerspezies ein homochiraler Komplex aus zwei gauche-Monomeren beobachtet. ${ }^{46,188}$ Mittels Mikrowellenspektroskopie gelang aber zusätzlich auch der Nachweis eines heterochiralen Dimers mit einem Anteil von etwa $10 \%$ in Heliumexpansionen von $0.5 \%$ Trifluorethanol in 2-4 bar Helium durch eine $0.8 \mathrm{~mm}$ Lochdüse. ${ }^{236}$ Möglicherweise begünstigt diese Düsengeometrie die Bildung des weniger stabilen heterochiralen Dimers gegenüber den Expansionen im Filet-Jet. Auf das heterochirale Dimer wurden mittels Ramanspektroskopie nur schwache Hinweise in Heliumexpansionen gefunden. ${ }^{188}$ Zwar zeigt das O-H Streckschwingungsspektrum in Abbildung 5.10 schwache Signale bei $3571 \mathrm{~cm}^{-1}$ und $3588 \mathrm{~cm}^{-1}$, die auch in Ramanspektren zu erkennen sind. ${ }^{188}$ Jedoch kommen sie auf Grund ihrer Verschiebungen zum Monomer nicht als Banden des heterochiralen Dimers in Frage, sondern sind eventuell eher heißen Banden zuzuordnen. Aus der Struktur der Trimersignale wurde geschlossen, dass vermutlich verschiedene Trimerstrukturen in Heliumexpansionen von Trifluorethanol vorliegen. ${ }^{188}$

Rein experimentell lässt sich im Ferninfrarotbereich nur eine Bande als Clusterbande charakterisieren. Sie liegt bei $476 \mathrm{~cm}^{-1}$ und ihr Wachstumsverhalten ähnelt den Trimer- und Tetramerbanden in den entsprechenden $\mathrm{O}-\mathrm{H}$ Streckschwingungsspektren. Tatsächlich wird in den berechneten und skalierten Clusterspektren bei dieser Position eine Trimerbande mit ähnlicher Intensität vorhergesagt. Diese Vorhersagen wurden für das homochirale Dimer und das stabilste Trimer ${ }^{188}$ durchgeführt. Die Wellenzahlen aus den harmonischen Vorhersagen auf B3LYP-D3/6$311+\mathrm{G}(2 \mathrm{~d}, \mathrm{p})-\mathrm{Niveau}$ für die Cluster wurden mit den Faktoren 0.965 im O-H Streckschwingungsbereich und 0.849 im Ferninfrarotbereich skaliert. Die Faktoren entsprechen den Mittelwerten aus den Faktoren, die im Falle von Methanol und tert-Butylalkohol zur Skalierung in diesen Spektralbereichen verwendet wurden (Vgl. Kapitel 5.2.1 und 5.10). Zur Vorhersage der Intensitäten im Ferninfrarotbereich wurden die berechneten Intensitäten in Abbildung 5.10 so skaliert, dass sie für beide Clustergrößen im $\mathrm{O}-\mathrm{H}$ Streckschwingungsbereich mit dem unteren Jetspektrum übereinstimmen. Dabei wurde für das Trimer angenommen, dass die gesamte Trimersignalintensität im $\mathrm{O}-\mathrm{H}$ Streckschwingungsspektrum vom stabilsten Trimer ${ }^{188}$ stammt. Die unskalierten Wellenzahlen und Intensitäten sind in Tabelle A.10 im Anhang gegeben. Benachbart zu der möglichen Trimerbande bei $476 \mathrm{~cm}^{-1}$ liegt ein schwaches Absorptionssignal bei 485 bis $490 \mathrm{~cm}^{-1}$ das von einem weniger stabilen Trimer oder größerem Cluster stammen könnte. Außerdem könnte zu diesem schwachen Signal der Oberton der O-H Torsion beitragen. Für diesen Übergang wird ein durch Tunneln aufgespaltenes Signal bei 492 und $514 \mathrm{~cm}^{-1}$ vorhergesagt. ${ }^{231}$

Eine weitere intensive Bande in den FTIR-Jetspektren bei $396 \mathrm{~cm}^{-1}$ lässt sich auf Grund der experimentellen Befunde nicht zuordnen. An dieser Position ist bisher kein Monomerübergang bekannt ${ }^{224}$ und auch das Ramanspektrum zeigt an dieser Stelle keine Intensität. Zwar sollte in diesem Bereich die Kombinationsbande aus $\mathrm{CF}_{3}$ Torsion und $\mathrm{O}-\mathrm{H}$ Torsion liegen $\left(116 \mathrm{~cm}^{-1}+283 \mathrm{~cm}^{-1}=399 \mathrm{~cm}^{-1}\right)$, jedoch ist die Intensität dieser Bande zu hoch für diesen als schwach vorhergesagten Übergang $\left(1.3 \frac{\mathrm{km}}{\mathrm{mol}}\right)$. Auch eine Verstärkung durch Fermiresonanz kann ausgeschlossen werden, da im Ramanspektrum keine Intensität in diesem Bereich vorliegt. Ge- 
gen eine Zuordnung zu Clustern spricht das Wachstumsverhalten dieser Bande, dass eher dem des Monomers ähnelt. Jedoch könnte durch die Nähe dieser Bande bei $396 \mathrm{~cm}^{-1}$ zur Transmissionslücke des Strahlteilers die Intensität verfälscht werden. Für das untere Jetspektrum wird an dieser Stelle genau die $\mathrm{O}-\mathrm{H}$ Librationsschwingung des Dimerakzeptors, der sich selbst in einer schwachen $\mathrm{O}-\mathrm{H} \cdots \mathrm{F}$ Wasserstoffbrücke befindet, mit sehr ähnlicher Intensität vorhergesagt. Für die Spektren bei anderen Expansionsbedingungen würde sich jedoch eine schlechtere Übereinstimmung zwischen experimenteller und vorhergesagter Intensität ergeben. Zumal die Intensität der Dimerakzeptorlibration bei geringeren Konzentrationen und Stagnationsdrücken deutlich geringer ausfallen sollte, eine Zuordnung zu größeren Clustern ist aber noch unwahrscheinlicher. Statt an dieser Position könnte der Übergang der Dimerakzeptorlibration jedoch auch mit einem Monomerübergang in der Nähe überlappen oder im Bereich der Transmissionslücke des Strahlteilers liegen.

Die weiteren Signale des Dimers und Trimers könnten im Bereich von 521 bis $534 \mathrm{~cm}^{-1}$ liegen, bei dem ein sehr breiter Bereich mit schwacher Absorption vorliegt. Außerdem könnte die Übergänge der Dimerdonorlibration und der weiteren Librationen des Trimers mit der intensiven Monomerbande bei $548 \mathrm{~cm}^{-1}$ überlappen. In einer Neon-Matrix zeigen sich breite Absorptionsbereiche bei 550 und $380 \mathrm{~cm}^{-1}$, die auf die Librationen des Dimerdonors und Dimerakzeptors zurück geführt werden können. ${ }^{219}$ In der warmen Gasphase wurden die gemischten Komplexe von Trifluorethanol mit Wasserstoffbrückenakzeptoren untersucht. ${ }^{214}$ Dabei wurde die Libration im gemischten Dimer mit Propanal im Bereich von 500 bis $600 \mathrm{~cm}^{-1}$ gefunden, ${ }^{214}$ was auf eine ähnlich starke Wasserstoffbrücke wie im Trifluorethanoldimer hindeutet. In Komplexen mit Ammoniak $^{214}$ liegt die Libration bei ungefähr $660 \mathrm{~cm}^{-1}$, was auch durch eine Änderung der Konformation von Trifluorethanol beeinflusst werden sollte. ${ }^{232}$ Eine noch stärkere Wasserstoffbrücke scheint im Dimer mit Trimethylamin vorzuliegen, dessen Librationsübergang bei 725 bis $800 \mathrm{~cm}^{-1}$ zugeordnet wurde. ${ }^{214}$ Diese systematische Erhöhung der Wellenzahlen der Librationsübergänge geht mit einer Vergrößerung der beobachteten $\mathrm{O}-\mathrm{H}$ Streckschwingungsverschiebungen einher. ${ }^{214}$

\subsubsection{Trifluorethanol-OD}

In Abbildung 5.11 ist ein Jetspektrum von Trifluorethanol-OD in Helium gezeigt. Durch die Deuterierung der Hydroxylgruppe ändert sich nicht nur die Bandenposition der Torsionsanregung deutlich, auch der Übergang der asymmetrischen $\mathrm{F}-\mathrm{C}-\mathrm{C}-\mathrm{O}$ Knickschwingungen ist an deutlich anderer Position zu finden. Im Vergleich zur undeuterierten Spezies liegt die Hydroxylschwingung $38 \mathrm{~cm}^{-1}$ tiefer bei $245 \mathrm{~cm}^{-1}$. Die vorherige Zuordnung dieses Übergangs ${ }^{224}$ bei 189 bis $195 \mathrm{~cm}^{-1}$ kann auf Grund der anharmonischen Vorhersage (B3LYP-D3/6-311+G(2d,p)) bei $242 \mathrm{~cm}^{-1}$ und der Bandenposition in einer Ar-Matrix ${ }^{234}$ bei $239 \mathrm{~cm}^{-1}$ widerlegt werden. Stattdessen sollte in dem Bereich von 189 bis $195 \mathrm{~cm}^{-1}$ die asymmetrischen $\mathrm{F}-\mathrm{C}-\mathrm{C}-\mathrm{O}$ Knickschwingungen liegen, die damit um etwa $40 \mathrm{~cm}^{-1}$ tiefer liegt als in der undeuterierten Spezies. Dies folgt aus der anharmonischen Analyse, die diese Schwingung bei $193 \mathrm{~cm}^{-1}$ vorhersagt. Die Positionen der übrigen Fundamentalübergänge von Trifluorethanol (s.Tabelle 5.10) ändern sich dagegen nur geringfügig durch die Deuterierung der Hydroxylfunktion. Signale des undeuterierten Monomers wie der intensiven $\mathrm{O}-\mathrm{H}$ Torsion lassen sich dagegen nicht beobachten, was die 


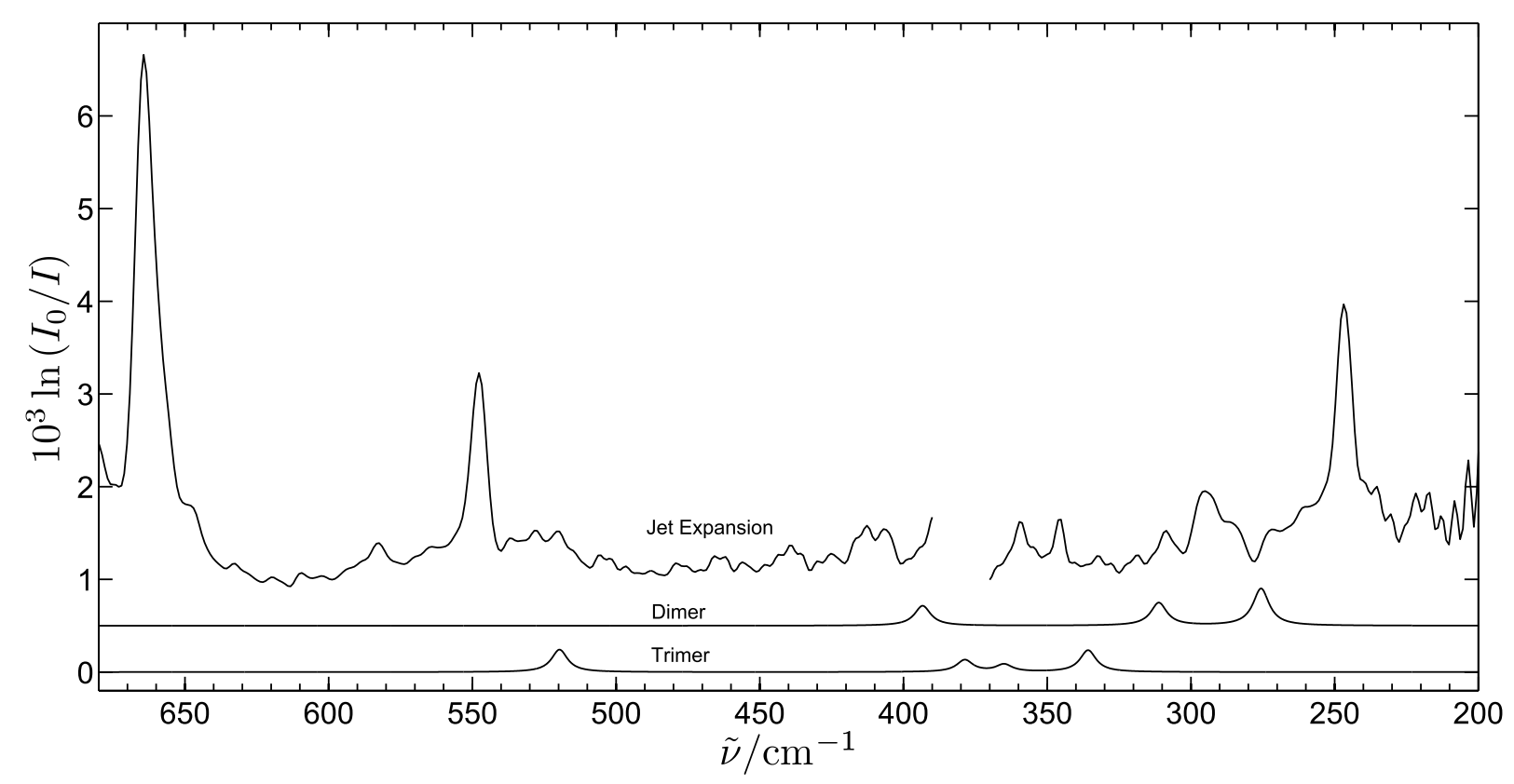

Abbildung 5.11.: Vergleich der FTIR-Spektren von Trifluorethanol-OD in Jetexpansionen des Filet-Jet mit berechneten Clusterspektren. Das FTIR-Spektrum zeigt Expansionen von $0.3 \%$ Trifluorethanol-OD in 0.8 bar Helium (120 Pulse). In den berechneten Spektren sind nur die Übergänge gezeigt, deren Normalmoden in der harmonischen Schwingungsanalyse (B3LYP-D3/6-311+G(2d,p)) einen deutlichen O-D Torsionsanteil zeigen. Ihre Wellenzahlen (B3LYP-D3/6-311+G(2d,p)) wurden mit demselben Faktor wie in Abbildung 5.10 skaliert. Die Clusterkonzentrationen wurden mit Hilfe von Trifluorethanol-OH Jetspektren unter ähnlichen Bedingungen im $\mathrm{O}-\mathrm{H}$ Streckschwingungsbereich abgeschätzt und die vorhergesagten Intensitäten für jede Clustergröße entsprechend skaliert.

signifikante Deuterierung des gemessenen Gasgemisches aufzeigt.

Neben den intensiven Monomerübergängen im Ferninfrarotspektrum von Trifluorethanol lassen sich noch einige schwächere Übergänge erkennen. Der Vergleich mit den harmonischen Vorhersagen der Clusterspektren hilft bei der Zuordnung. Zur Verbesserung der Übersichtlichkeit, sind in Abbildung 5.11 nur Übergänge gezeigt, die einen signifikanten Anteil an O-D Torsion zeigen. Dafür liegt zugrunde, dass die geringe Intensität der $\mathrm{O}-\mathrm{D}$ Übergänge in der harmonischen Vorhersage teilweise in der gleichen Größenordnung wie die der intramolekularen Gerüstmoden ist, für welche aber die vorgenommene Skalierung mit dem Faktor 0.849 auf Grund ihrer geringeren Anharmonizität keinen Sinn ergeben würde. Die Intensitäten wurden auf Grundlage der O-H Streckschwingungsspektren der undeuterierten Spezies bei ähnlichen Expansionsbedingungen unter Annahme gleicher Konzentration der einzelnen Clustergrößen wie in Abbildung 5.10 skaliert. Die unskalierten Wellenzahlen und Intensitäten sind in Tabelle A.10 im Anhang gegeben. Einer relativ intensiven Bande bei $295 \mathrm{~cm}^{-1}$ könnte so die Akzeptorlibration des Dimers zugeordnet werden. In der berechneten Vorhersage, ergeben sich für diese Schwingung 
Tabelle 5.10.: Bandenpositionen der Übergänge von Trifluorethanol-OD Monomer und Clustern im Ferninfrarotbereich in $\mathrm{cm}^{-1}$ : Experimentelle Ergebnisse aus FTIR-Messungen von Trifluorethanol in He-Jetexpansionen und IR-Absorptionsmessungen in der Gasphase. ${ }^{224}$ Zusätzlich zu den berechneten Wellenzahlen aus einer anharmonischen Schwingungsrechnung auf B3LYP-D3/6-311+G(2d,p)-Niveau sind die Intensitäten in $\frac{\mathrm{km}}{\mathrm{mol}}$ in runden Klammern gegeben. Die Zuordnungen von Übergängen, die unsicher sind (siehe Text), sind in eckigen Klammern geschrieben. Die Fundamentalübergänge des Monomers sind konsistent mit der Analyse dieser Moden in Ar-Matrizen ${ }^{234}$ zugeordnet.

\begin{tabular}{|c|c|c|c|}
\hline $\begin{array}{c}\text { FTIR- } \\
\text { Jetspektrum }\end{array}$ & $\begin{array}{c}\text { IR- } \\
\text { Gasphase }^{224}\end{array}$ & $\begin{array}{c}\text { B3LYP-D3 } \\
\text { 6-311+G(2d,p) } \\
\text { anharmonisch }\end{array}$ & Zuordnung \\
\hline 245 & 189,195 & $242(39)$ & $\mathrm{O}-\mathrm{H}$ Torsion \\
\hline 295 & & & [Dimerakzeptorlibration] \\
\hline 309 & & $300(0.02)$ & {$\left[\mathrm{CF}_{3}\right.$ Tors. + asymm. $\mathrm{F}-\mathrm{C}-\mathrm{C}-\mathrm{O}$ Knick $]$} \\
\hline 346 & & & [Trimerlibration] \\
\hline 360 & 360 & $353(6)$ & $\mathrm{CF}_{3}$ Schaukelschw. \\
\hline 407 & & & [Dimerdonor] \\
\hline 414 & 412 & $402(3)$ & symm. $\mathrm{F}-\mathrm{C}-\mathrm{C}-\mathrm{O}$ Knick \\
\hline $515-537$ & & $520(0.4)$ & $\mathrm{CF}_{3}$ Deformation \\
\hline 548 & 543 & $530(7)$ & $\mathrm{CF}_{3}$ Deformation \\
\hline 583 & & & [Clusterlibration] \\
\hline 663 & & $641(24)$ & $\mathrm{CF}_{3}$ Deformation \\
\hline
\end{tabular}

auf Grund harmonischer Modenmischung mit der schwächeren $\mathrm{CF}_{3}$ Schaukelschwingung des Akzeptors zwei intensive Übergänge. Eine Entstörung von dieser Mischung sollte zu einer Bandenposition der Akzeptorlibration zwischen den beiden vorhergesagten Übergängen mit deutlich höherer Intensität führen. Dies würde zu einer besseren Übereinstimmung zur beobachteten Position und Intensität der Bande bei $295 \mathrm{~cm}^{-1}$ führen. Damit würde sich für die Wellenzahlen der Übergänge der Akzeptorlibration ein Isotopenverhältnis $\frac{\tilde{\nu}_{\mathrm{OH}}}{\tilde{\nu}_{\mathrm{OD}}}$ von 1.34 ergeben, das nah an den entsprechenden Werten der Wasserstoffbrücken-gebundenen Hydroxylgruppen in Methanolclustern (Kapitel 5.2.3) ist und so auf die korrekte Zuordnung der Akzeptorbanden der deuterierten und undeuterierten Spezies hinweist. In der anharmonischen Realität ist eine Resonanz mit der $\mathrm{CF}_{3}$ Schaukelschwingung nicht zu erwarten, da der Energieunterschied zwischen den Übergängen deutlich größer als in der harmonischen Vorhersage ausfällt. Der Dimerdonorübergang wird in der Nähe der Transmissionslücke des Strahlteilers vorhergesagt und könnte in diesem Bereich liegen. Es wäre aber auch möglich, dass er zu dem energetisch niedrigen Signal der Doppelbande bei 407 und $414 \mathrm{~cm}^{-1}$ beiträgt. Bei $414 \mathrm{~cm}^{-1}$ kann die symmetrische $\mathrm{F}-\mathrm{C}-\mathrm{C}-\mathrm{O}$ Knickschwingung des Monomers zugeordnet werden.

Einer relativ schmalen Bande bei $346 \mathrm{~cm}^{-1}$ könnte die Trimerlibration mit niedrigster Wellenzahl zugeordnet werden. Die Trimerlibration bei mittlerer Energie befindet sich in der harmonischen Rechnung in Resonanz mit der symmetrischen $\mathrm{F}-\mathrm{C}-\mathrm{C}-\mathrm{O}$ Knickschwingung und sollte 


\section{5. $\mathrm{O}-\mathrm{H}$ Torsionen}

im Bereich der Transmissionslücke des Strahlteilers liegen. Der Übergang der Trimerlibration bei der höchsten Wellenzahl könnte zu dem breiten Bereich bei 515 bis $537 \mathrm{~cm}^{-1}$ beitragen, bei dem im Spektrum nur wenig Intensität zu finden ist. Außerdem sollte dort auch der Monomerübergang der $\mathrm{CF}_{3}$ Deformation mit schwächster Intensität liegen.

Es verbleiben zwei schwache Banden bei $309 \mathrm{~cm}^{-1}$ und $583 \mathrm{~cm}^{-1}$ zur Zuordnung. Bei ersterer könnte es sich um die Kombinationsbande aus $\mathrm{CF}_{3}$ Torsion und asymmetrischer $\mathrm{F}-\mathrm{C}-\mathrm{C}-\mathrm{O}$ Knickschwingung handeln, die auch im Spektrum des undeuterierten Monomers als möglicher Ursprung eines schwachen Signals zugeordnet wurde (s.o.). Die ungefähren Positionen der Monomerübergänge ${ }^{224}$ mit $113 \mathrm{~cm}^{-1}$ und 189 bis $195 \mathrm{~cm}^{-1}$ würden $\mathrm{zu}$ der Bandenposition bei $309 \mathrm{~cm}^{-1}$ passen. Der schwache Übergang bei $583 \mathrm{~cm}^{-1}$ geht möglicherweise auf die Libration eines größeren Clusters zurück. Die O-deuterierten Tetramere von Methanol $\left(559 / 566 \mathrm{~cm}^{-1}\right)$ und tert-Butylalkohol $\left(571 \mathrm{~cm}^{-1}\right)$ zeigen an ähnlicher Position einen intensiven Übergang ihrer Libration. Je nach Geometrie und Symmetrie könnte das Trifluorethanoltetramer ein ähnliches Bandenmuster zeigen wie Tetramere der zwei aliphatischen Alkohole, allerdings wäre dann eine niedrigere Wellenzahl des intensiven Übergangs wegen der schwächeren Wasserstoffbrücken zu erwarten, wie es auch für die Dimerdonorlibration der Fall sein sollte.

\subsection{Methyllaktat}

In Jetexpansionen liegt das Monomer von Methyllaktat überwiegend in einer Konformation vor, die eine intramolekulare Wasserstoffbrücke zur Carbonylfunktion aufweist. ${ }^{49,237}$ Die Cluster von Methyllaktat wurden bisher vor allem im O-H Streckschwingungsbereich untersucht. ${ }^{45,238-240}$ Dabei spielt Chiralitätserkennung eine wichtige Rolle für die Struktur der Spektren und der relativen Häufigkeit der verschiedenen Clusterstrukturen in Jetexpansionen. Vor allem die Bevorzugung einer homochiralen Trimer- und einer heterochiralen Tetramerstruktur in Jetexpansionen ist hervorzuheben. Die Strukturen der bevorzugten Dimere konnten dagegen bisher noch nicht vollständig aufgeklärt werden.

In Abbildung 5.12 sind die Spektren von Methyllaktat in Jetexpansionen am Filet-Jet gezeigt. Die Spektren im $\mathrm{O}-\mathrm{H}$ Streckschwingungsbereich und im oberen Bereich der $\mathrm{O}-\mathrm{H}$ Torsion $\left(\geq 550 \mathrm{~cm}^{-1}\right)$ wurden von Susanne Hesse gemessen und analysiert. ${ }^{63}$ Zusätzlich sind die Spektren aus Ferninfrarotmessungen $\left(200-680 \mathrm{~cm}^{-1}\right)$ gezeigt. Bei dem Vergleich dieser Spektren, die mit gleichen Sättigertemperaturen und Stagnationsdrücken aufgenommen wurden, zeigen sich allerdings inkonsistente Substanzkonzentrationen. Mit Hilfe von Gasphasenspektren, die im Anschluss an die Jetexpansionen von Methyllaktat im Filet-Jet gemessen wurden, lassen sich jedoch die tatsächlichen Substanzkonzentrationen zwischen den einzelnen Messreihen vergleichen und übereinstimmende Bedingungen finden. Deshalb sind in Abbildung 5.12 Spektren gezeigt, deren Konzentration auf Grundlage des Dampfdruckes von Methyllaktat unterschiedlich sind, die tatsächlichen Substanzkonzentrationen im Reservoir sind jedoch in allen Spektren nahezu identisch. Gezeigt sind die Spektren von Heliumexpansionen von reinem $S$-Methyllaktat mit homochiralen Aggregaten und die Spektren von Heliumexpansionen des Racemats mit homochiralen und heterochiralen Aggregaten. Das synthetische heterochirale Dimerspektrum ergibt sich, wenn das Spektrum des Racemats mit dem Faktor zwei multipliziert und anschließend das 

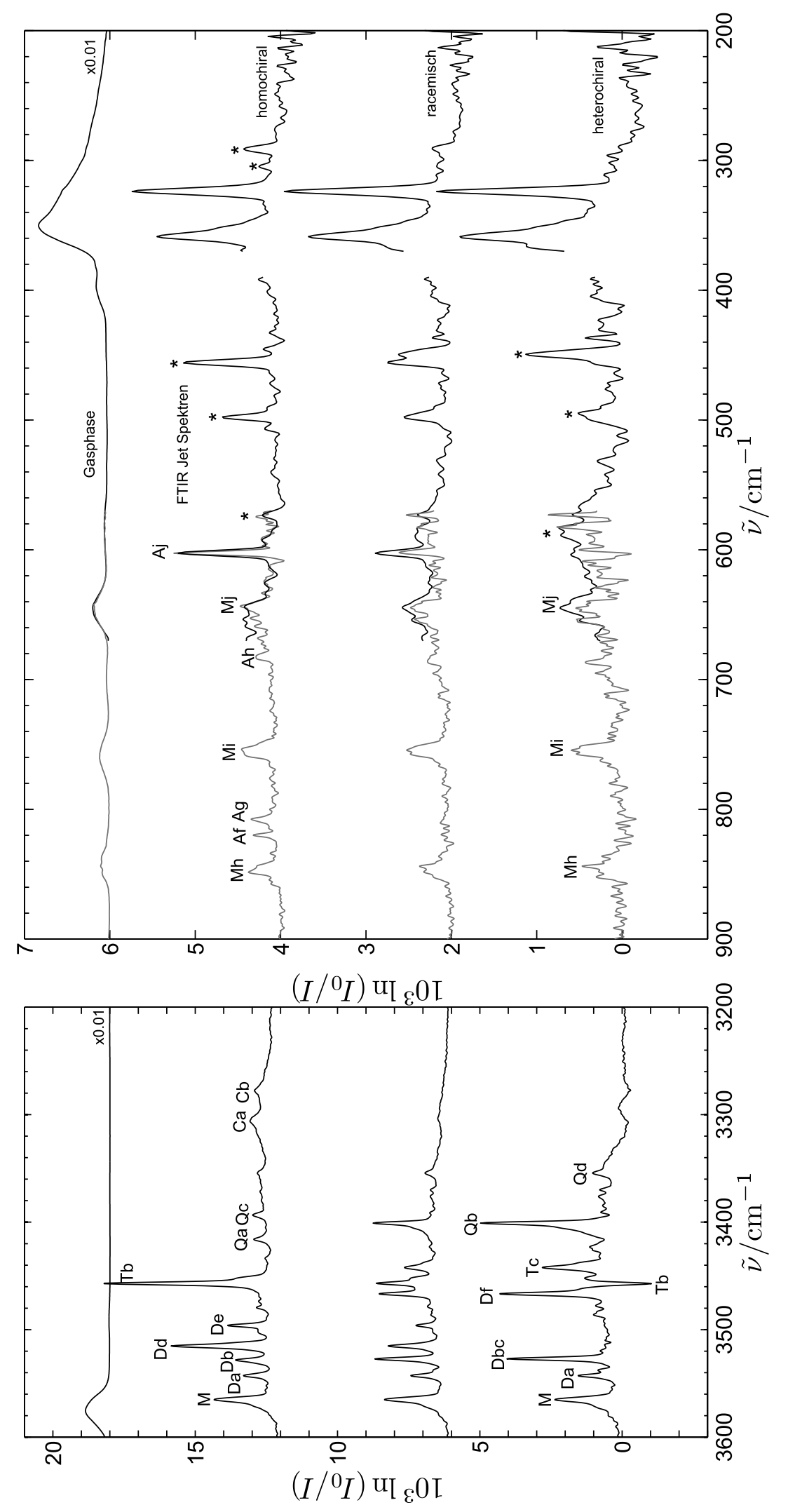

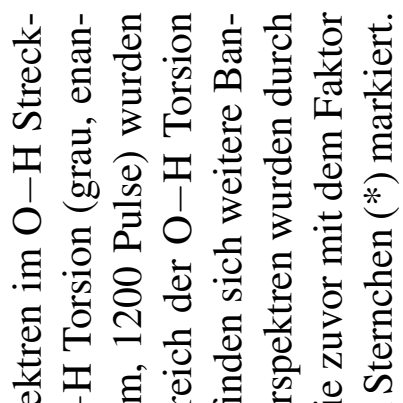

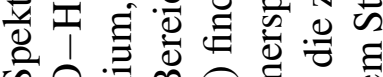
की

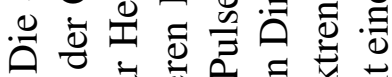

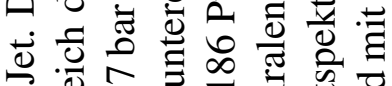

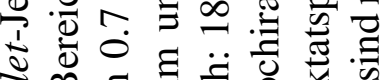
运

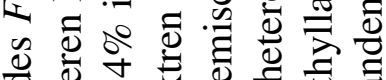

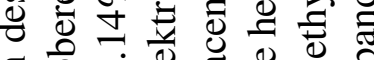

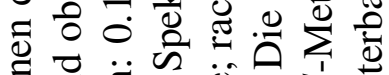

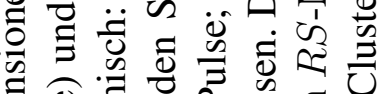

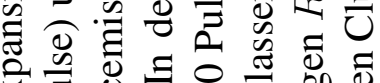

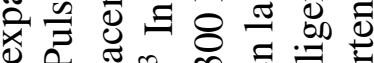
$\stackrel{0}{0}$

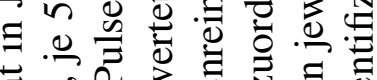

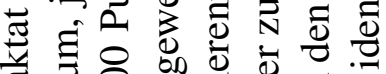

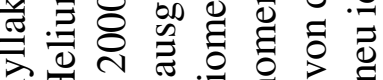

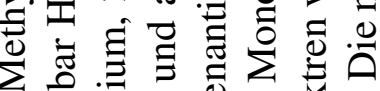
궁 웡

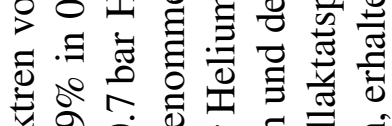

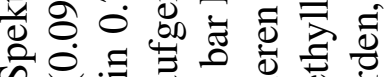

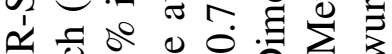

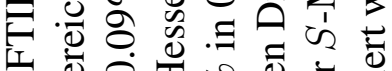
I Ð

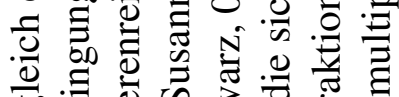

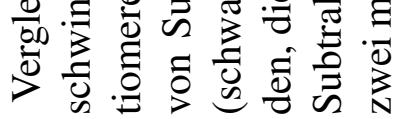

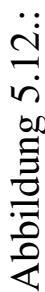




\section{5. $\mathrm{O}-\mathrm{H}$ Torsionen}

Spektrum der enantiomerenreinen Substanz subtrahiert wird. Unter der Annahme, dass sich die Dimere rein statistisch bilden, sollten somit im heterochiralen Dimerspektrum nur Signale von heterochiralen Dimeren sichtbar sein.

Die Monomerbanden lassen sich zum einen durch den Vergleich mit dem Gasphasenspektrum in Abbildung 5.12 identifizieren. Zum anderen sollten sie sich beim Vergleich der Spektren von $S$-Methyllaktat mit dem Racemat nicht unterscheiden. Dies trifft zu für die Banden Mh, Mi und $\mathrm{Mj}$ die bereits als Monomerbanden zugeordnet wurden. ${ }^{63}$ Zusätzlich zeigen sich zwei Banden im unteren Ferninfrarotbereich, deren Position und Intensität in den Spektren von enantiomerenreinem und racemischem Methyllaktat ebenfalls identisch sind. Beide liegen im Bereich einer breiten Bande im Spektrum der warmen Gasphase. Durch die intramolekulare Wasserstoffbrücke sollten die Schwingungen der O-H Gruppe besonders temperatursensitiv sein, da eine höhere Temperatur eine Schwächung der Wasserstoffbrücke bedeutet. Für die O-H Streckschwingung bedeutet das eine um etwa $10 \mathrm{~cm}^{-1}$ höhere Wellenzahl des Absorptionsmaximums ihrer Bande in der warmen Gasphase gegenüber der Jetexpansion. Dagegen sollte sich für die Bande der $\mathrm{O}-\mathrm{H}$ Torsion in der Gasphase eine tiefere Position des Absorptionsmaximums ergeben als in der Jetexpansion. Da das Maximum des breiten Absorptionsbereichs in der Gasphase bei $350 \mathrm{~cm}^{-1}$ liegt, ist die Zuordnung der Bande bei $358 \mathrm{~cm}^{-1}$ im Jetspektrum zur O-H Torsion naheliegend. Die Banden $\mathrm{Mh}$ bis $\mathrm{Mj}$ zeigen deutlich geringere Temperatureffekte mit Wellenzahlunterschieden von maximal $3 \mathrm{~cm}^{-1}$. Die intensive Bande bei $324 \mathrm{~cm}^{-1}$ in den Jetspektren verhält sich ebenfalls wie die übrigen Monomerbanden und an ihrer Position zeigt sich in der Gasphase eine sehr kleine Schulter. Jedoch fällt die Schmalheit dieser Bande, die sie eher den scharfen Clusterbanden in den Jetspektren ähneln lässt, auf. Die anderen gezeigten Monomerbanden sind in den Spektren der Jetexpansionen deutlich breiter, was an ihrer rudimentären Rotationsstruktur liegt. Jedoch zeigen sich in Jetexpansionen auch recht scharfe Monomerbanden, wie etwa die der $\mathrm{C}-\mathrm{OCH}_{3}$ Streckschwingung. ${ }^{62}$ Andererseits gibt es auch im $\mathrm{O}-\mathrm{H}$ Streckschwingungsbereich eine Dimerbande (Da), die keinerlei Unterschied zwischen den Spektren von enantiomerenreinem und racemischem Methyllaktat zeigt. Anhand der vorliegenden Messdaten lässt sich also nicht genau klären, ob es sich bei der Bande bei $324 \mathrm{~cm}^{-1}$ um eine Monomer- oder Dimerbande handelt. Eine Untersuchung der Konzentrationsabhängigkeit dieser Bande sollte dies jedoch leicht aufklären können. Quantenchemische Rechnungen auf B3LYP-D3/6-311+G(2d,p)-Niveau sagen für das Monomer in diesem niedrigen Wellenzahlbereich zwei intensive Banden voraus. In der harmonischen Analyse wird die $\mathrm{O}-\mathrm{H}$ Torsion bei $371 \mathrm{~cm}^{-1}$ vorhergesagt. Zusätzlich ergibt sich in der Rechnung eine Gerüstschwingung bei $354 \mathrm{~cm}^{-1}$. Somit werden also zwei Monomerschwingungen erwartet, jedoch deutet die große Abweichung zwischen experimentellen und vorhergesagten Wellenzahlen darauf hin, dass die Schwingungen einen deutlichen anharmonischen Beitrag beinhalten. Die übrigen Monomerschwingungen $\mathrm{Mh}$ bis $\mathrm{Mj}$ können anhand der Schwingungsrechnung als Gerüstschwingungen charakterisiert werden.

Die Clusterbanden lassen sich leicht durch die Unterschiede zwischen den Spektren der enantiomerenreinen und racemischen Substanz identifizieren. Im Spektrum der homochiralen Aggregate zeigen sich neben den bekannten Signalen ${ }^{63}$ zwei intensive Banden bei 456 und $498 \mathrm{~cm}^{-1}$. Da sich ihre Intensität im Spektrum des Racemats halbiert, können sie den homochiralen Dimeren zugeordnet werden, deren Banden im $\mathrm{O}-\mathrm{H}$ Streckschwingungsbereich das gleiche Verhalten zeigen. Im synthetischen heterochiralen Dimerspektrum zeigen sich die Signale von heterochira- 
Tabelle 5.11.: Bandenpositionen der Übergänge von Methyllaktatmonomere im niederen Infrarotbereich in $\mathrm{cm}^{-1}$ : Experimentelle Ergebnisse aus FTIR-Messungen von Methyllaktat in He-Jetexpansionen und IR-Absorptionsmessungen in einer ArgonMatrix. Zusätzlich zu den berechneten Wellenzahlen aus einer harmonischen Schwingungsrechnung des Monomers auf B3LYP-D3/6-311+G(2d,p)-Niveau sind die Intensitäten in $\frac{\mathrm{km}}{\mathrm{mol}}$ in runden Klammern gegeben. Die Zuordnungen werden im Text erläutert.

\begin{tabular}{cccl}
\hline $\begin{array}{c}\text { FTIR- } \\
\text { Jetspektrum }\end{array}$ & $\begin{array}{c}\text { Argon } \\
\text { Matrix }{ }^{241}\end{array}$ & $\begin{array}{c}\text { B3LYP-D3 } \\
\text { 6-311+G(2d,p) }\end{array}$ & Zuordnung \\
\hline 324 & & $354(46)$ & [Gerüst-Biegeschwingung oder Cluster] \\
358 & & $371(73)$ & O-H Torsion \\
$644^{63}$ & $654-623$ & $652(13)$ & $\mathrm{Mj},{ }^{63}$ Gerüstschwingung \\
$756^{63}$ & 758 & $764(10)$ & $\mathrm{Mi},{ }^{63}$ Gerüstschwingung \\
$848^{63}$ & 844 & $853(6)$ & $\mathrm{Mh},{ }^{63}$ Gerüstschwingung \\
\hline
\end{tabular}

len Clustern leicht verschoben bei 449 und $497 \mathrm{~cm}^{-1}$. Der geringe Wellenzahlunterschied deutet darauf hin, dass es sich bei ihnen ebenfalls um Dimersignale handelt. Außerdem finden sich in den Spektren noch schwächere Clustersignale. Diese zeigen sich im enantiomerenreinen Spektrum bei 291, 305 und $573 \mathrm{~cm}^{-1}$. Das heterochirale Dimerspektrum zeigt eine breite Clusterabsorption um $586 \mathrm{~cm}^{-1}$.

Die bekannten Clustersignale Af bis Aj konnten bisher noch keiner konkreten Clustergröße zugeordnet werden, für Ah kommen jedoch nur Cluster größer als das Dimer in Frage. ${ }^{63}$ Auch für die hier nochmals gemessene Bande $\mathrm{Aj}$ ist die Zuordnung nicht eindeutig. Während die hier gezeigten Spektren für den Bereich 200-680 $\mathrm{cm}^{-1}$ auf Grund der Halbierung der Intensität im Spektrum des Racemats eher auf einen Dimerursprung hindeuten, zeigen analoge hier nicht gezeigte Spektren, die mit KBr Optiken im Bereich $400-680 \mathrm{~cm}^{-1}$ gemessen wurden, eher einen Trimercharakter dieser Bande auf. Auch die Spektren, die von Susanne Hesse gemessen wurden, ${ }^{63}$ deuten eher auf einen Trimerursprung hin.

In Abbildung 5.13 sind die stabilsten gefundenen Clusterstrukturen der Dimere (bis $7 \mathrm{~kJ} / \mathrm{mol}$ über dem globalen Minimum), Trimere und Tetramere auf B3LYP-D3/6-311+G(2d,p) Niveau und ihre Dissoziationsenergien einschließlich Nullpunktskorrektur dargestellt. Ihre unskalierten harmonischen Wellenzahlen und Intensitäten sind in Tabelle A.11 im Anhang gegeben. Für die Tetramere konnten die bisher bekannten Ergebnisse auf anderen Rechenniveaus qualitativ reproduziert werden, ${ }^{45,63,238,239,242}$ während sich für die Trimere und Dimere teils abweichende oder neue Strukturen ergeben. Die Nomenklatur der Dimer- und Trimerstrukturen richtet sich nach Referenz 63. Bei neuen Strukturen wurde lediglich die Laufzahl erhöht. Die Benennung der Tetramerstrukturen richtet sich nach ihrem Strukturmotiv.

Das stabilste Tetramer ist die heterochirale Struktur Qb, die der gleichnamigen O-H Streckschwingungsbande in den Jetspektren des Racemats zugeordnet wurde. ${ }^{45,239,242}$ Sie weist eine RSRS Anordnung der einzelnen Moleküle und vier einzelne $\mathrm{O}-\mathrm{H} \cdots \mathrm{O}=\mathrm{C}$ Wasserstoffbrücken auf. ${ }^{45,63,239,242}$ Das gleiche Bindungsmotiv der Wasserstoffbrücken lässt sich auch bei einem homochiralen Tetramer finden (Qhom), ${ }^{62}$ jedoch ist eine Strukur mit vier O-H $\cdots \mathrm{O}-\mathrm{H}$ Wasser- 

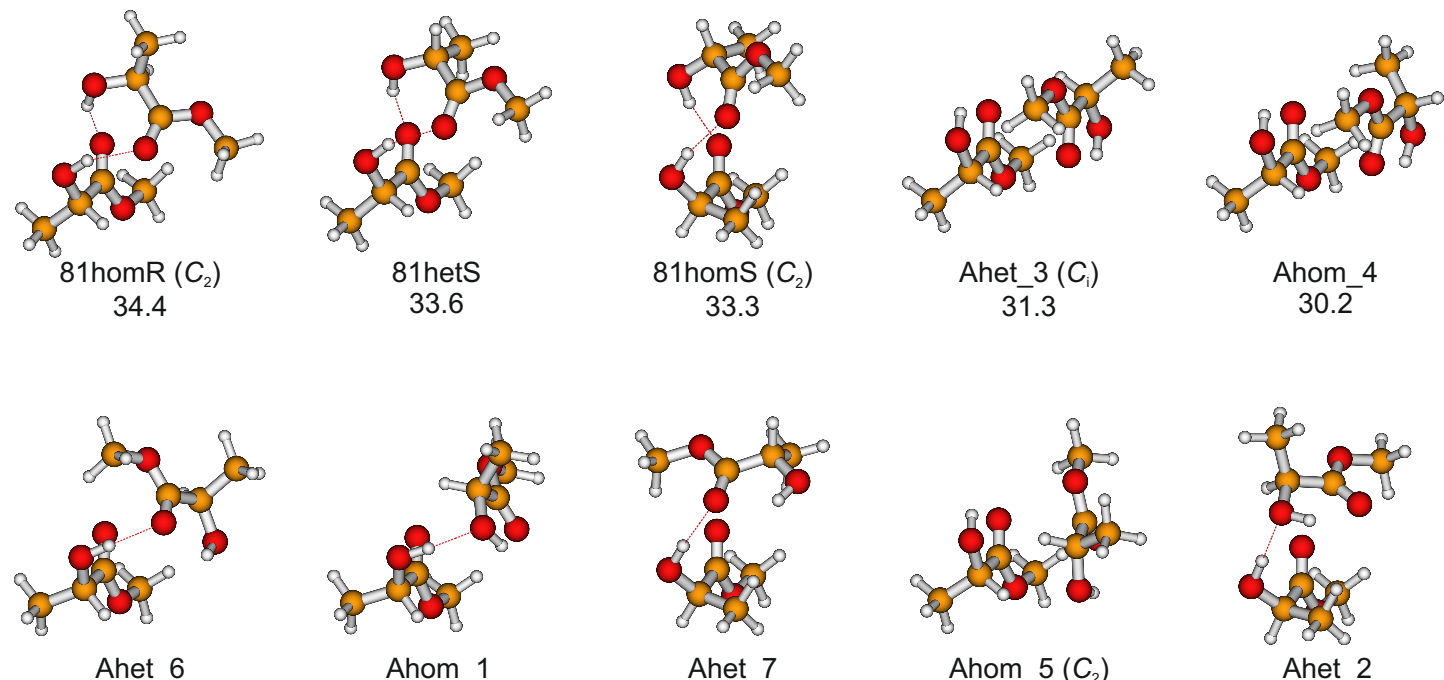

Ahet 6

Ahom_1

Ahet 7

29.3

Ahet 2
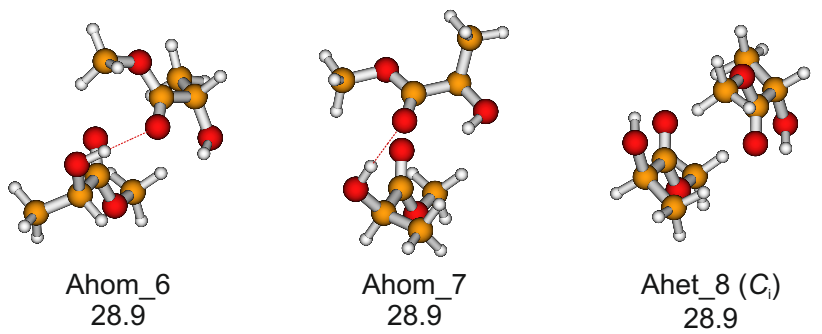

Ahom_6

28.9

Ahet_8 $\left(C_{\mathrm{i}}\right)$

28.9

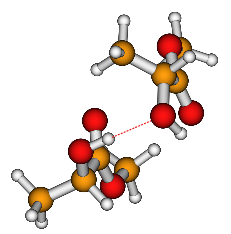

Ahet 1
$27 . \overline{8}$

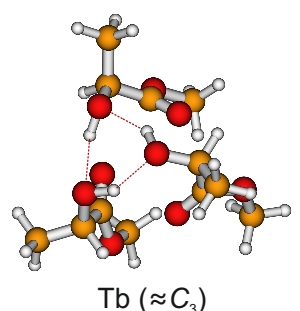

81.7

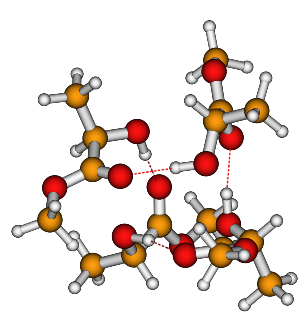

Qb $\left(S_{4}\right)$

133.2

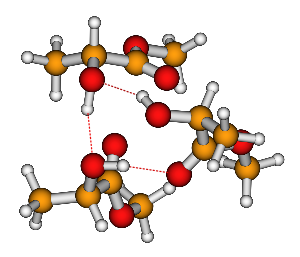

Thet 2

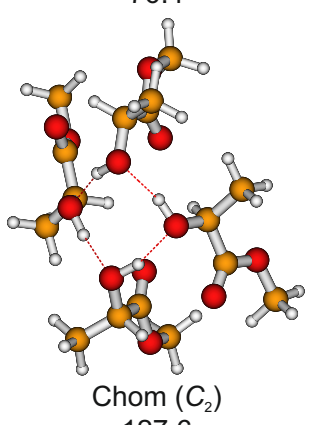

127.6

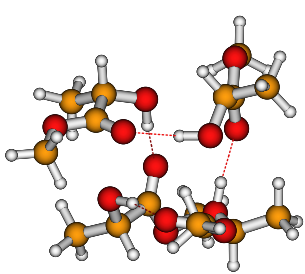

Qhom $\left(C_{2}\right)$

121.6

Abbildung 5.13.: Berechnete Clusterstrukturen von Methyllaktat auf B3LYP-D3/6-311+G(2d,p) Niveau und Dissoziationsenergien $D_{0}$ in $\mathrm{kJ} / \mathrm{mol}$ unter Berücksichtigung der harmonischen Nullpunktsenergien. 
stoffbrücken in einer kooperativen Ringanordnung (Chom) deutlich stabiler ${ }^{45,239,242}$ und kann den homochiralen Tetramerbanden $\mathrm{Ca}$ und $\mathrm{Cb}$ zugeordnet werden. ${ }^{45,239,242}$ Die homochiralen Tetramerstrukturen in Referenz 63 sind dagegen noch weniger stabil als Qhom.

Der markanten IR-aktiven Trimerbande Tb und einer stärker verschobenen ramanaktiven Trimerbande in den Spektren der homochiralen Cluster kann die Trimerstruktur Tb auf Grund ihrer (hier näherungsweisen) $C_{3}$ Symmetrie zugeordnet werden. ${ }^{45,62,238,242}$ In den hier durchgeführten Rechnungen und auf einigen anderen Rechenniveaus stellt es sich als stabilstes Trimer dar. ${ }^{63}$

Für das stabilste heterochirale Trimer wurde bisher auf einigen Rechenniveaus eine analoge Ringstruktur gefunden. ${ }^{62,63}$ Auf B3LYP-D3/6-311+G(2d,p)-Niveau konnte eine solche Anordnung jedoch nicht reproduziert werden. Stattdessen ergibt sich in Übereinstimmung mit Referenz 242 als stabilstes heterochirales Trimer (Thet2) eine abweichende Struktur, bei der ein $\mathrm{O}-\mathrm{H} \cdots \mathrm{O}-\mathrm{H} \cdots \mathrm{O}-\mathrm{H} \cdots \mathrm{O}=\mathrm{C}$ Wasserstoffbrückensystem vorliegt.

Im Falle der Dimere ergibt sich als stabilste Struktur das homochirale 81homR, das auch auf anderen Rechenniveaus die stabilste bisher gefundene Dimergeometrie aufweist. ${ }^{62,63,242} 81$ homR ist das einzige Dimer für das bisher eine schlüssige Zuordnung zur IR-aktiven Bande Dd und einer benachbarten Raman-aktiven Bande existiert. ${ }^{240}$ Energetisch folgen darauf analog zu Referenz 242 und den DFT-Ergebnissen in Referenz 63 die bekannten Strukturen ${ }^{62,63,238,242} 81$ hetS (heterochiral) und 81homS (homochiral), die wie 81 homR zwei einzelne $\mathrm{O}-\mathrm{H} \cdots \mathrm{O}=\mathrm{C}$ Wasserstoffbrücken aufweisen. Weiterhin wurden Assoziate, bei denen keine intermolekularen Wasserstoffbrücken vorliegen, gefunden, die teilweise schon bekannt sind: Ahet_3, ${ }^{62,63,242}$ Ahom_4, Ahom_5 ${ }^{242}$ und Ahet_8. Neu sind Strukturen, die ein antikooperatives $\mathrm{O}-\mathrm{H} \cdots \mathrm{O} \cdots \mathrm{H}-\mathrm{O}$ Wasserstoffbrückensystem aufweisen aber energetisch auf B3LYP-D3/6-311+G(2d,p)-Niveau trotzdem konkurrenzfähig sind: Ahet_6, Ahet_7, Ahom_6 und Ahom_7. Dagegen weisen die bekannten ${ }^{63}$ Strukturen Ahom_1, Ahet_2 und Ahet_1 kooperative Wechselwirkungen zwischen einer intra- und einer intermolekularen Wasserstoffbrücke auf.

In den Abbildungen 5.14 und 5.15 werden die Jetspektren von Methyllaktat mit quantenchemischen Frequenzvorhersagen verglichen. Dabei wurden die berechneten Wellenzahlen der $\mathrm{O}-\mathrm{H}$ Streckschwingungen so skaliert, dass experimentelle und vorhergesagte Monomerposition übereinstimmen (Faktor 0.9566). Im Ferninfrarotbereich wurden zwei unterschiedliche Faktoren zur Skalierung der berechneten Wellenzahlen verwendet. Die Wellenzahlen von Strukturen, die ausschließlich intramolekulare Wasserstoffbrücken aufweisen, wurden mit dem Faktor 0.965 skaliert, der sich aus experimenteller und vorhergesagter Position der $\mathrm{O}-\mathrm{H}$ Torsion des Monomers ergibt. Unter der Annahme, dass die Bande Aj von einem Trimer stammt und somit als intensive Librationsbande von Tb identifiziert werden kann, ergibt sich aus gemessener und vorhergesagter Position der Trimerlibration ein Skalierungsfaktor von 0.9095. Dieser wurde zur Skalierung der Wellenzahlen aller Clusterstrukturen, die zwischenmolekulare Wasserstoffbrücken aufweisen, verwendet. Die vorhergesagten Intensitäten jeder Clusterstruktur wurden so skaliert, dass sie im $\mathrm{O}-\mathrm{H}$ Streckschwingungsbereich die gleiche Intensität wie die nächstliegenden experimentellen Banden derselben Clustergröße aufweisen.

Der Vergleich der Frequenzvorhersagen für die homochiralen Cluster mit den Spektren des $\mathrm{O}-\mathrm{H}$ Streckschwingungsbereich (Abbildung 5.14) zeigt im Falle der Tetramere und der Trimere gute Übereinstimmung. Die vorhergesagte harmonische Wellenzahlverschiebung von $-123 \mathrm{~cm}^{-1}$ der intensiven Bande des homochiralen Trimers Tb zum Monomerübergang ist nur leicht grö- 

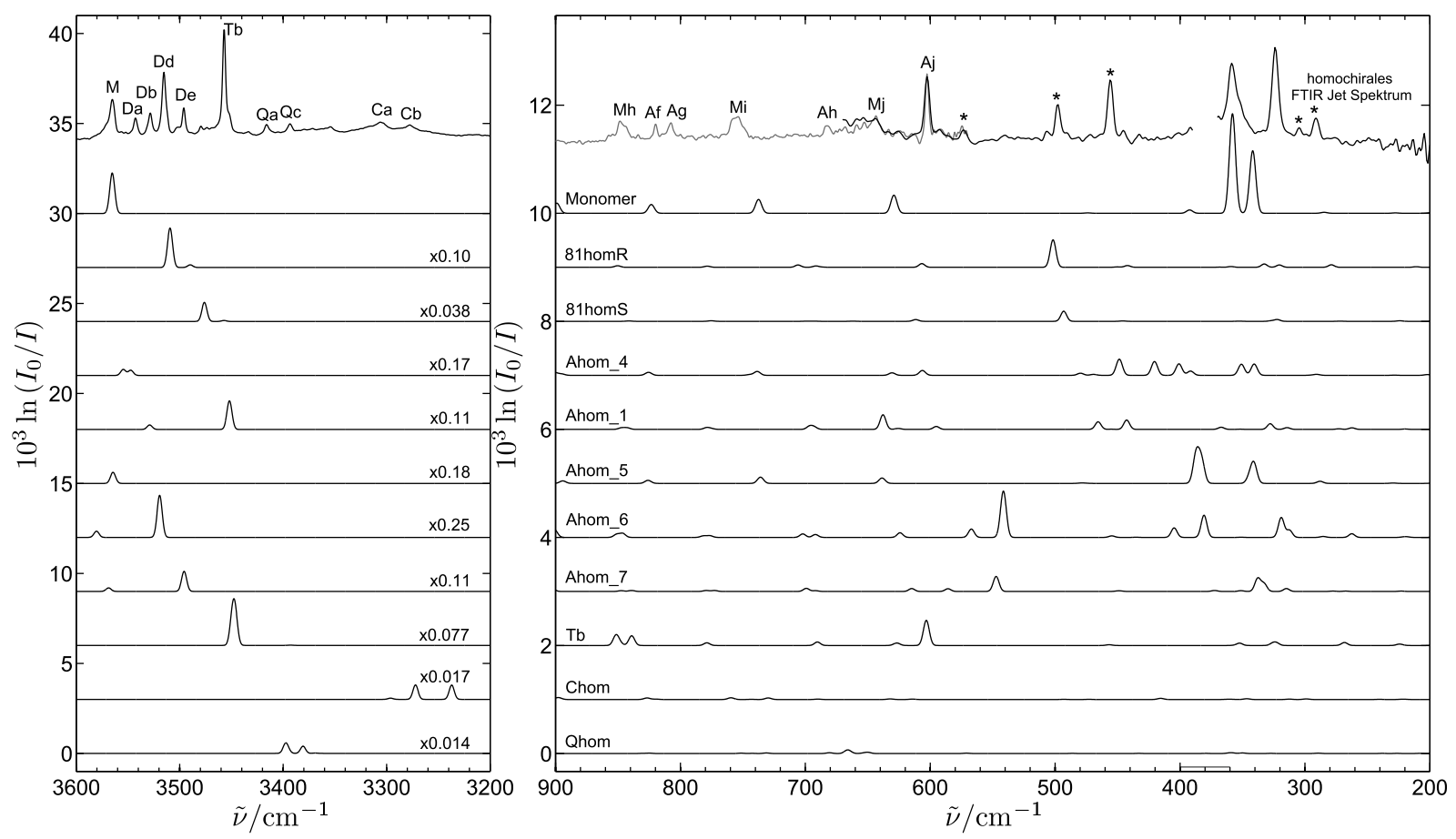

Abbildung 5.14.: Vergleich der enantiomerenreinen FTIR-Spektren von Methyllaktat in Jetexpansionen des Filet-Jet mit berechneten Spektren von homochiralen Clustern. Im O-H Streckschwingungsbereich wurden die Wellenzahlen der harmonischen Schwingungsrechnungen (B3LYP-D3/6-311+G(2d,p)) mit dem Faktor 0.9566 skaliert, der die berechnete und experimentelle Bandenposition des Monomers in Übereinstimmung bringt. Im Ferninfrarotbereich wurden die Wellenzahlen der Cluster, die zwischenmolekulare Wasserstoffbrücken aufweisen, mit dem Faktor 0.9095 skaliert. Die Wellenzahlen von Monomer, Ahom_4 und Ahom_5 wurden mit dem Faktor 0.965 skaliert. Die berechneten Intensitäten wurden für jede Clustergeometrie so skaliert, dass die berechneten Intensitäten im $\mathrm{O}-\mathrm{H}$ Streckschwingungsbereich etwa zu den experimentellen Banden passen, die am nächsten zu den vorhergesagten Bandenpositionen liegen. Die Skalierungsfaktoren der Intensitäten für die einzelnen Clusterspezies sind relativ zur Skalierung des Monomers in der linken Hälfte der Abbildung gegeben. Die experimentell gefundenen Clusterbanden sind im Ferninfrarotbereich mit einem Sternchen $(*)$ markiert.

Ber als die experimentelle Verschiebung ${ }^{63}$ von $-108 \mathrm{~cm}^{-1}$. Auch die harmonischen Vorhersagen zu den homochiralen Tetrameren Chom und Qhom überschätzen die Wellenzahlverschiebung der intensiven Banden gegenüber den experimentellen Werten. Jedoch lassen sich die unterschiedlich starken Verschiebungen der Tetramerbanden auf die strukturellen Unterschiede der Tetramergeometrien zurückführen. Der kooperative Ring in Chom sorgt für eine stärkere Wellenzahlverschiebung der $\mathrm{O}-\mathrm{H}$ Streckschwingungsübergänge und führt zu der Zuordnung zu den 

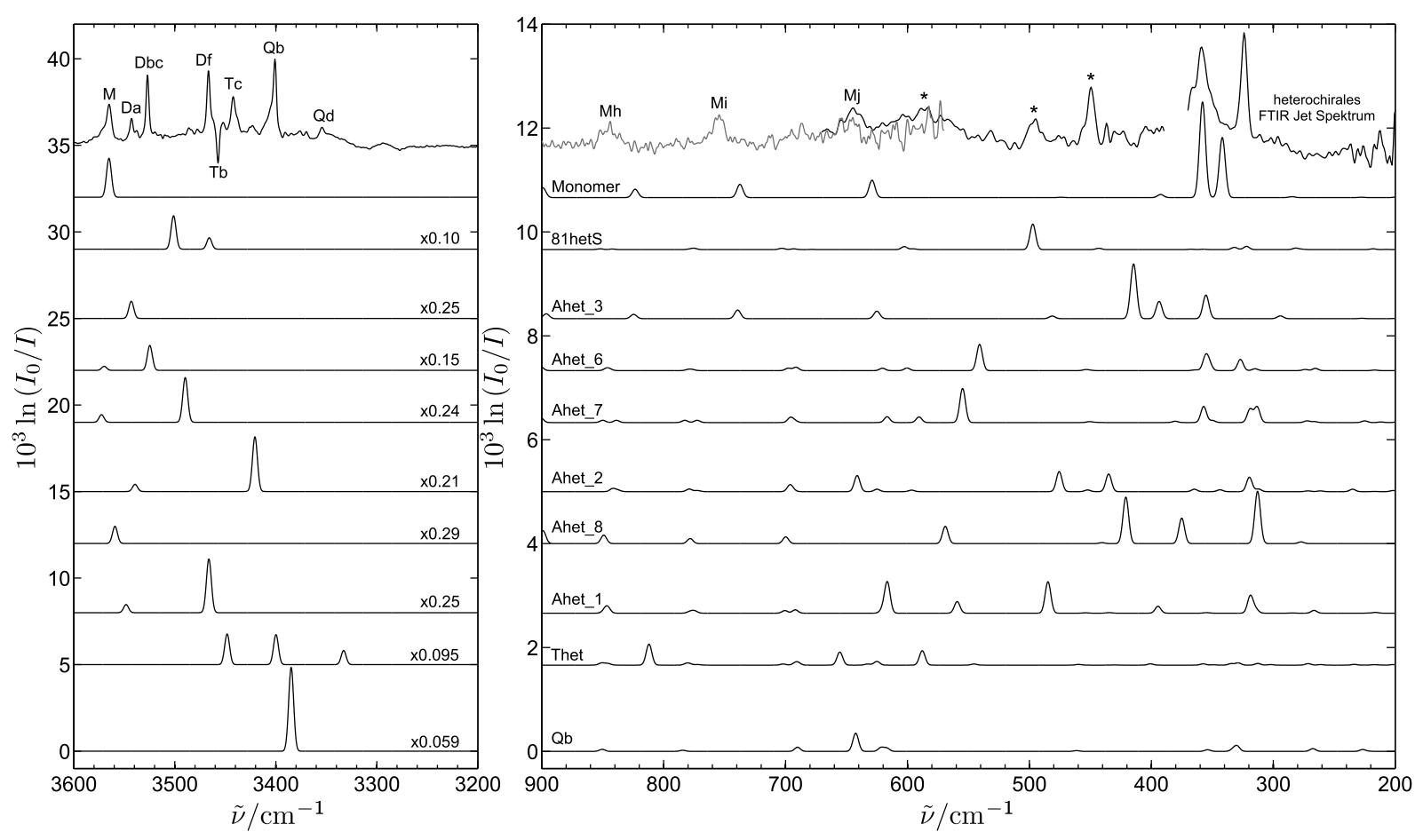

Abbildung 5.15.: Vergleich der synthetischen „heterochiralen“FTIR-Spektren von Methyllaktat in Jetexpansionen des Filet-Jet mit berechneten Spektren von heterochiralen Clustern. Es wurde die gleiche Skalierung der berechneten Wellenzahlen und Intensitäten wie in Abbildung 5.14 vorgenommen. Die Wellenzahlen des Monomers sowie von Ahet_3 und Ahet_8 wurden im Ferninfraroten mit dem Faktor 0.965 skaliert. Die berechneten Intensitäten wurden für jede Clustergeometrie so skaliert, dass die berechneten Intensitäten im O-H Streckschwingungsspektrum etwa zu den experimentellen Banden passen, die am nächsten zu den vorhergesagten Bandenpositionen liegen. Die Skalierungsfaktoren für die einzelnen Clusterspezies sind relativ zur Skalierung des Monomers in der linken Hälfte der Abbildung gegeben. Die experimentell gefundenen Clusterbanden sind im Ferninfrarotbereich mit einem Sternchen $(*)$ markiert.

experimentellen Banden $\mathrm{Ca}$ und Cb. ${ }^{45,239,242}$ Die vier isolierten Wasserstoffbrücken in Qhom führen dagegen zu einer geringeren Verschiebung der Übergänge gegenüber dem Monomer und legen so die Zuordnung zu den experimentellen Banden Qa und Qc nahe.

Für die homochiralen Dimere ergeben sich aus dem Vergleich mit den Spektren keine neuen Erkenntnisse. Die vorhergesagte Verschiebung der IR-intensiven Bande des Dimers 81homR überschätzt die experimentelle Verschiebung der ihm zugeordneten Bande $\operatorname{Dd}^{240}$ etwas. Für die meisten anderen homochiralen Dimerstrukturen lassen sich Dimerbanden finden, die den vorhergesagten Verschiebungen etwa entsprechen würden. Nur die Assoziate Ahom_4 und Ahom_5 stimmen weniger gut mit dem experimentellen Spektrum überein. Ein Dublett wie für Ahom_4 zeigt sich an entsprechender Stelle in den Spektren nicht und Hinweise darauf, dass die Mono- 


\section{5. $\mathrm{O}-\mathrm{H}$ Torsionen}

merbande ein Dimersignal überlagert, wie es für Ahom_5 erwartet werden würde, lassen sich ebenfalls nicht finden.

Für den Vergleich der vorhergesagten Clusterübergänge mit den Spektren im Ferninfrarotbereich (Abbildung 5.14) wurde angenommen, dass die intensivste Librationsbande des homochiralen Trimers $\mathrm{Tb}$ der Bande $\mathrm{Aj}$ zuzuordnen ist. Da der Trimerursprung von $\mathrm{Aj}$ experimentell möglich aber nicht sicher ist, käme alternativ auch eine Zuordnung von Aj zur Libration des Dimers Ahom_6 in Frage. Dafür spricht die hohe Intensität die für die Librationsübergänge von Ahom_6 erwartet wird. Als Gegenargument dafür ist aber die vorhergesagte Wellenzahl anzuführen. In harmonischer Näherung ergibt sich eine unskalierte Position bei $595 \mathrm{~cm}^{-1}$, die sehr nah an der experimentellen Position von $603 \mathrm{~cm}^{-1}$ liegt. Auf Grund der großen diagonalen Anharmonizität von Librationsschwingungen ${ }^{14}$ sollte die anharmonische Bandenposition von Ahom_6 deutlich tiefer liegen, als $595 \mathrm{~cm}^{-1}$. Für die Zuordnung von Aj zum Trimer Tb spricht auch, dass die daraus resultierende Skalierung zu einer guten Übereinstimmung der vorhergesagten Librationsposition des einzig nachgewiesenen Dimers 81homR mit der Dimerbande bei $498 \mathrm{~cm}^{-1}$ führt.

Für die Banden Af und Ag ergibt sich als wahrscheinlichste Zuordnung die höherenergetische Libration des Trimers Tb, wobei die Dublettstruktur in der harmonischen Vorhersage auf Modenmischung zurückzuführen ist. Insofern könnte auch nur eine der Banden Af und Ag auf das Trimer zurückzuführen sein.

Auf Grund ihrer geringen Konzentration in den Jetexpansionen werden für die Tetramere keine intensiven Signale erwartet. Einzig die intensivste Librationsbande von Qhom könnte der experimentellen Bande Ah zugeordnet werden, da für diese Cluster größer als dem Dimer zugeordnet wurden ${ }^{63}$ und vorhergesagte Intensität und Position nach Skalierung mit dem Spektrum etwa übereinstimmen.

Wie bereits beschrieben passt die skalierte harmonische Vorhersage zu 81 homR sowohl bezüglich der Intensität als auch der Wellenzahl gut zur Dimerbande bei $498 \mathrm{~cm}^{-1}$. Andere Dimere ließen sich dieser Bande nur zuordnen, wenn ihre Konzentrationen deutlich höher lägen, als sich aus den Spektren des $\mathrm{O}-\mathrm{H}$ Streckschwingungsbereichs ableiten lässt. Tatsächlich lassen sich die erwarteten Signale aus den harmonischen Vorhersagen zu den meisten Dimerstrukturen nicht mit dem Spektrum im Ferninfrarotbereich vereinbaren. Neben 81 homR ließe sich nur Ahom_7 ohne weiteres dem schwachen Clustersignal bei $573 \mathrm{~cm}^{-1}$ zuordnen. Für 81 homS und Ahom_6 müssten zumindest Konzentrationen angenommen werden, die von den aus dem $\mathrm{O}-\mathrm{H}$ Streckschwingungsbereichs angenommenen abweichen, um eine Übereinstimmung mit den gemessenen Intensitäten zu erreichen. Für Ahom_4 und Ahom_1 deuten die vorhergesagten Bandenpositionen darauf hin, dass diese Strukturen nicht in signifikanter Konzentration in den Jetexpansionen des Filet-Jet vorliegen. Ahom_5 lässt sich auf Grund der geringen spektralen Differenzen zum Monomer weder ausschließen noch verifizieren. Außerdem sind in Abbildung 5.14 die Skalierungsfaktoren für die Intensitäten relativ zum Monomer angegeben. Da am Filet-Jet typischerweise Konzentration von Alkoholdimeren von etwa $10 \%$ beobachtet werden, erscheinen die Skalierungsfaktoren von Ahom_4, Ahom_5 und Ahom_6 zu hoch, erfordern also Konzentrationen von deutlich über $10 \%$ gegenüber dem Monomer um zur Erklärung der $\mathrm{O}-\mathrm{H}$ Streckschwingungssignale herangezogen werden zu können. Dies spricht ebenfalls gegen das Vorhandensein dieser Dimere in den Jetexpansionen des Filet-Jet.

Besonders hervorzuheben ist, dass keines der vorhergesagten Clusterspektren eine Erklärung 
für die Dimerbande bei $456 \mathrm{~cm}^{-1}$ liefert. Es gibt jedoch die Möglichkeit, die Dimerbanden in beiden Spektralbereichen der enantiomerenreinen Substanz als Signale einer einzigen Dimerspezies zu interpretieren. Dafür bietet sich 81 homR an. So könnte man das Dimerbandenmuster im $\mathrm{O}-\mathrm{H}$ Streckschwingungsbereich erklären, wenn ein Franck-Condon-artiger Effekt analog zum Methanoltrimer ${ }^{36}$ angenommen wird. Folglich würde mehr Intensität 81 homR zuzuordnen sein und sich daraus eine höhere Konzentration des Dimers ergeben. Im Ferninfrarotbereich ließe sich ebenfalls eine höhere Intensität zu 81homR finden, wenn neben der Bande bei $498 \mathrm{~cm}^{-1}$ auch die Bande bei $456 \mathrm{~cm}^{-1}$ zu dieser Dimerstruktur zugeordnet werden würden. Dieses Dublett lieBe sich mit einer Fermiresonanz erklären, da nur eine IR-intensive Librationsbande für 81homR erwartet wird. Dadurch würde die entstörte Bandenposition der Librationsbande deutlich tiefer liegen als $498 \mathrm{~cm}^{-1}$, dies wäre aber noch mit der hohen Anharmonizität von Librationsschwingungen ${ }^{14}$ vereinbar.

Im O-H Streckschwingungsbereich des heterochiralen Dimerspektrums ergeben sich beim Vergleich mit den harmonischen Vorhersagen (Abbildung 5.15) zu den heterochiralen Dimeren keine eindeutigen Zuordnungen. Das vorhergesagte Spektrum zu 81 hetS variiert sehr stark mit der verwendeten Rechenmethode zwischen B3LYP, B3LYP-D3 (mit oder ohne Becke-Johnson Dämpfung) und MP2. Dies zeigt, das die harmonische Vorhersage für diese Dimerstrukutur sehr ungenau ist und möglicherweise stark von dem Dispersionsanteil an der Wechselwirkung zwischen den Molekülen in den Rechnungen abhängt. Die vorhergesagten Verschiebungen für die Strukturen Ahet_7 und Ahet_2 sprechen eher gegen ihr Vorhandensein in den Jetexpansionen des Filet-Jet. Ahet_3, Ahet_6 und Ahet_1 lassen sich dagegen mit den Spektren im O-H Streckschwingungsbereich vereinbaren. Ahet_8 würde wahrscheinlich mit dem Monomer überlagern, wofür sich in den experimentellen Spektren keine Hinweise finden lassen.

Das vorhergesagte Spektrum für Thet_2 mit drei intensiven Peaks, lässt sich nicht mit dem experimentellen Befund einer einzelnen heterochiralen Trimerbande vereinbaren und spricht klar gegen das Vorhandensein von Thet_2 in den Jetexpansionen. Dagegen passt die vorhergesagte Bandenposition des heterochiralen Tetramers $\mathrm{Qb}$ sehr gut mit der gleichnamigen experimentellen Bande überein.

Der Vergleich der vorhergesagten Spektren der heterochiralen Cluster im Ferninfrarotbereich mit dem synthetischen Spektrum der heterochiralen Cluster aus den Experimenten fällt in Abbildung 5.15 auf Grund des schlechteren Signal-zu-Rauschen Verhältnisses des letzteren schwierig aus. Eine Übereinstimmung der Librationsbanden von Thet_2 wird auf Grund der Diskrepanz im $\mathrm{O}-\mathrm{H}$ Streckschwingungsbereich weder erwartet noch vorgefunden. Für das Tetramer $\mathrm{Qb}$ wird ein intensiver Librationsübergang im Bereich der Monomerbande Mj erwartet, der möglicherweise auf Grund von Überlappung im Spektrum jedoch nicht sichtbar ist. Für die Clusterbande bei $497 \mathrm{~cm}^{-1}$ bietet sich vor allem die Zuordnung zu 81hetS, weniger Ahet_1, für das noch ein intensiver Peak bei höherer Wellenzahl erwartet wird, an. Für die Clusterbande bei $449 \mathrm{~cm}^{-1}$ kämen die Anlagerungsstrukturen Ahet_3 und Ahet_8 in Frage, wenn auch ihre Bandenpositionen nach Skalierung (mit dem Faktor aus der Monomervorhersage) etwas zu gering erscheinen. Der breite Absorptionsbereich von Clustern bei $586 \mathrm{~cm}^{-1}$ käme vor allem zur Zuordnung zum heterochiralen Trimer in Betracht. Für dieses wurde jedoch bisher keine mit den Spektren kompatible und energetisch günstige Struktur beschrieben. Ferner könnten auch Ahet_6, Ahet_7 und Ahet_1 zu dem breiten Absorptionsbereich bei $586 \mathrm{~cm}^{-1}$ beitragen. Zu Ahet_2 lässt sich auf 


\section{5. $\mathrm{O}-\mathrm{H}$ Torsionen}

Grund der geringen Intensität der vorhergesagten Signale keine eindeutige Aussage über das Vorhandensein in den Jetexpansionen treffen.

Zusammenfassend lässt sich feststellen, dass sich aus der Auswertung des Ferninfrarotbereichs nur begrenzt neue Erkenntnisse zu den Dimeren von Methyllaktat gewinnen lassen. Die Vorhersagen zu 81homR und Ahom_7 sind kompatibel mit den Spektren, während sich die meisten anderen homochiralen Dimerstrukturen auf Grundlage der harmonischen Rechnungen eher ausschließen lassen. Dass sich die homochirale Dimerbande bei $456 \mathrm{~cm}^{-1}$ keiner Dimerstruktur zuordnen lässt, weist auf ein Defizit bei den Rechnungen hin. Entweder ist die harmonische Rechnung auf B3LYP-D3/6-311+G(2d,p) Niveau nicht ausreichend, um die Librationssignale akkurat vorherzusagen (natürlich auch bezüglich einer möglichen Fermiresonanz) oder es fehlt in den Betrachtungen die Dimerstruktur, die diese Bande hervorruft. Im Falle der heterochiralen Dimere zeigt vor allem 81 hetS gute Übereinstimmung im Ferninfrarotbereich. Zur gleichen Bande ließe sich alternativ aber auch Ahet_1 zuordnen, das auch im $\mathrm{O}-\mathrm{H}$ Streckschwingungsbereich gute Übereinstimmung aufweist. Ausschließen lässt sich auf Grund der Ferninfrarotspektren keine der betrachteten heterochiralen Dimerstrukturen. Die Bande bei $324 \mathrm{~cm}^{-1}$ ist auf Grundlage der vorliegenden Dimerberechnung eher als Monomerbande zu identifizieren, da kein entsprechend intensives Dimersignal an dieser Position erwartet wird.

\subsection{Diskussion}

Nachdem in diesem Kapitel zahlreiche Librationsübergänge von Clustern beschrieben wurden, soll hier ein Vergleich zwischen den verschiedenen Systemen stattfinden. Generell wird für Librationsbanden eine Blauverschiebung gegenüber den Übergängen der freien $\mathrm{O}-\mathrm{H}$ Torsion beobachtet. Somit ist die $\mathrm{O}-\mathrm{H}$ Libration in ihrem Verhalten komplementär zur Rotverschiebung der $\mathrm{O}-\mathrm{H}$ Streckschwingung. Es ergibt sich aber keine so klare Clusterabfolge mit der Verschiebung für die Libration im Ferninfrarotbereich, da beispielsweise die Dimerdonorbanden der Alkohole zwischen den Trimerlibrationsübergängen liegen.

Generell ist die Blauverschiebung der Libration größer als die Rotverschiebung der $\mathrm{O}-\mathrm{H}$ Streckschwingung. Die einzige Ausnahme zeigt das Ameisensäuredimer. Dies wird ersichtlich aus Tabelle 5.12, in der die Verschiebung der IR-intensiven Clusterbanden verglichen werden. Weiterhin führen stärkere Wasserstoffbrücken in den Clustern ${ }^{41}$ auch zu größeren Blauverschiebungen, was man an den Beispielen Methanol und tert-Butylalkohol ableiten kann. Somit ergibt sich auch in den meisten Fällen eine Korrelation zwischen der Stärke der Rotverschiebung der Streckschwingung und der Blauverschiebung der Libration wenn man verschiedene Dimere, Trimere und Tetramere vergleicht. Für Dimere ist die Korrelation besonders deutlich (mit Ausnahme des Ameisensäuredimers): Die Blauverschiebung der Libration ist etwa 2.6 bis 2.9 mal größer als die Rotverschiebung der Streckschwingung. Für Trimere schwankt dieses Verhältnis der beiden Verschiebungen mehr, weist aber einen kleineren Faktor auf $\left(\frac{\Delta \tau_{\mathrm{OH}}}{-\Delta \nu_{\mathrm{OH}}}\right.$ in Tabelle 5.12). Für die Tetramere von Methanol und tert-Butylalkohol ist die Blauverschiebung der Libration nur 1.3 bis 1.4 mal größer als die Rotverschiebung der $\mathrm{O}-\mathrm{H}$ Streckschwingung.

Die anharmonischen Vorhersagen zu den Librationsübergängen weisen in allen Fällen eine Überschätzung der Wellenzahlen auf. Somit wird formal von der Rechnung die Kraft der Was- 
Tabelle 5.12.: Verschiebungen der IR-intensiven Clusterbanden zu den entsprechenden Monomerschwingungen für Dimerdonoren $\left(\mathrm{D}_{\mathrm{d}}\right)$, Trimere $(\mathrm{T})$ und Tetramere $(\mathrm{Q})$ verschiedener Systeme. Gegeben sind die Verschiebung der O-H Streckschwingung $\Delta \nu_{\mathrm{OH}}$ und der $\mathrm{O}-\mathrm{H}$ Torsion $\Delta \tau_{\mathrm{OH}}$ sowie die harmonischen und anharmonischen Vorhersagen zu $\Delta \tau_{\mathrm{OH}}$ auf B3LYP-D3/6-311+G(2d,p)-Niveau (alle in $\mathrm{cm}^{-1}$ ). AuBerdem ist das Verhältnis von Blauverschiebung der Torsion zur Rotverschiebung der Streckschwingung $\frac{\Delta \tau_{\mathrm{OH}}}{-\Delta \nu_{\mathrm{OH}}}$ gegeben.

\begin{tabular}{|c|c|c|c|c|c|}
\hline & \multirow[b]{2}{*}{$\Delta \nu_{\mathrm{OH}}$} & \multirow[b]{2}{*}{$\Delta \tau_{\mathrm{OH}}$} & \multirow{2}{*}{$\frac{\Delta \tau_{\mathrm{OH}}}{-\Delta \nu_{\mathrm{OH}}}$} & \multicolumn{2}{|c|}{ B3LYP-D3/6-311+G(2d,p) } \\
\hline & & & & $\Delta \tau_{\mathrm{OH}}$ harm. & $\Delta \tau_{\mathrm{OH}}$ anharm \\
\hline \multicolumn{6}{|l|}{$\mathrm{CH}_{3} \mathrm{OH}$} \\
\hline $\mathrm{D}_{\mathrm{d}}$ & $-111^{36}$ & $\approx+326^{a}$ & $\approx 2.9$ & +414 & +356 \\
\hline $\mathrm{T}$ & $-212,-217^{36}$ & $+320,+379$ & $1.5,1.7$ & $+372,+430$ & $+334,+420$ \\
\hline Q & -392 & $+529^{36}$ & 1.3 & +616 & \\
\hline \multicolumn{6}{|c|}{ tert-Butylalkohol-OH } \\
\hline $\mathrm{D}_{\mathrm{d}}$ & $-146^{37}$ & $\approx+380$ & $\approx 2.6$ & $\approx+450$ & \\
\hline $\mathrm{T}$ & $-190,-231^{37}$ & $\approx+330, \approx+400$ & $\approx 1.7$ & $\approx+350, \approx+450$ & \\
\hline Q & $-384^{37}$ & $\approx+540$ & $\approx 1.4$ & $\approx+600$ & \\
\hline \multicolumn{6}{|c|}{ Trifluorethanol-OH } \\
\hline $\mathrm{D}_{\mathrm{d}}$ & $-103^{188}$ & $\approx+270^{a}$ & $\approx 2.6$ & $\approx+290$ & \\
\hline $\mathrm{T}$ & $-155,-187^{188}$ & +193 & $1.2,1.0$ & $\approx+220$ & \\
\hline \multicolumn{6}{|c|}{ Methyllaktat } \\
\hline 81homR & $-50^{63}$ & +140 & 2.8 & $\approx+189$ & \\
\hline $\mathrm{Tb}$ & $-108^{63}$ & $+245^{63}$ & 2.3 & $\approx+300$ & \\
\hline \multicolumn{6}{|c|}{$\mathrm{CHOOH}$} \\
\hline $\mathrm{D}$ & $\approx-600^{15}$ & +299 & $\approx 0.5$ & +340 & +340 \\
\hline
\end{tabular}

${ }^{a}$ Wert aus Neonmatrix ${ }^{14,219}$

serstoffbrücke auf die O-H Torsion überschätzt. Dies könnte aber auch ein Problem der Schwingungsrechnung sein, die das Potential nur am Minimum in Betracht zieht und so die Anharmonizität der Schwingung nicht richtig erfasst. Die harmonische Analyse überschätzt die Blauverschiebung der Libration um 6 bis 35\%. Die starke Streuung weist darauf hin, dass sich die tatsächlichen Anharmonizitäten in den Systemen deutlich unterscheiden. 



\section{Ameisensäuredimer}
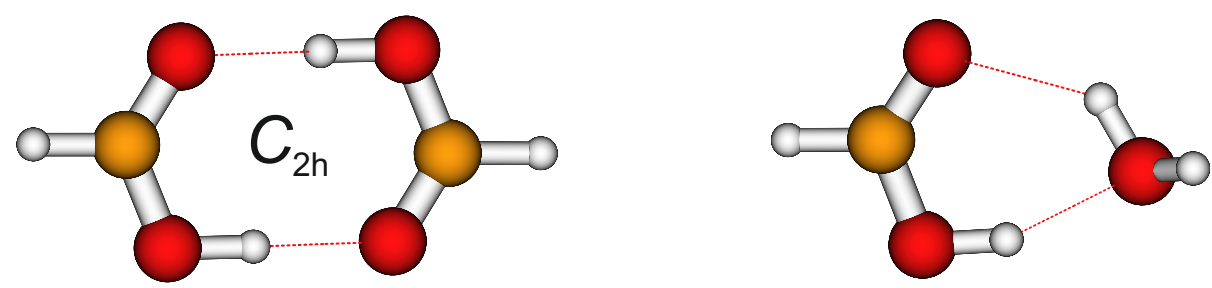

Abbildung 6.1.: Berechnete Strukturen (B3LYP-D3/6-311+G(2d,p)) des Ameisensäuredimers und des gemischten Dimers aus Ameisensäure und Wasser

Das Ameisensäuredimer weist eine cyclische Struktur mit zwei intermolekularen Wasserstoffbrücken und $C_{2 \mathrm{~h}}$ Symmetrie auf ${ }^{17,243}$ (s. Abbildung 6.1). Folglich sind aus dem Schwingungsgrundzustand nur Übergänge zu Niveaus mit $A_{u}$ und $B_{u}$ Symmetrie IR-aktiv, da sie die Inversionssymmetrie des Dimers aufheben. Ramanaktiv sind die Übergänge zu Zuständen mit $\mathrm{A}_{\mathrm{g}}$ und $\mathrm{B}_{\mathrm{g}}$ Symmetrie. Die ersten Obertöne können nur durch Ramanspektroskopie beobachtet werden, da diese Übergänge stets gerade Symmetrie aufweisen. Im Absorptionsspektrum lassen sich jedoch Kombinationstöne aus zwei Schwingungsquanten beobachten, wenn sie aus einer IR-aktiven und einer ramanaktiven Mode zusammengesetzt sind. Wenn sie dieselbe Symmetrie wie ein energetisch naheliegender Fundamentalübergang aufweisen, können Kombinationstöne zu Fermiresonanzen führen, die im IR Spektrum sichtbar werden. Die relativ großen Rotationskonstanten des Ameisensäuredimers führen dazu, dass sich bei ausreichend hoher Auflösung $\left(<1 \mathrm{~cm}^{-1}\right)$ die Rotationsstruktur der Dimerbanden in Form der $P, Q$ und $R$ Zweige am Filet-Jet beobachten lässt. Die Zuordnung der Banden zum $A, B$ oder $C$ Typ ${ }^{22}$ oder Mischtypen ermöglicht Rückschlüsse auf die Ausrichtung des Übergangsdipolmoments hinsichtlich der entsprechenden Rotationsachsen, ${ }^{20}$ was sich bei der Zuordnung der Banden in den Spektren als hilfreich erweist. Die zu erwartenden Bandentypen lassen sich aus quantenchemischen Rechnungen erhalten. Dazu wurden für die Isotopologen des Ameisensäuredimers anharmonische Frequenzvorhersagen auf B3LYP-D3/6-311+G(2d,p)-Niveau durchgeführt, auf die stets in diesem Kapitel - wenn im Text nicht anders beschrieben - Bezug genommen wird. Es wird hier die Notation für die Fundamentalübergänge des Ameisensäuredimers von Bertie et al. übernommen, ${ }^{244,245}$ die von anderen Notationen in der Literatur zu unterscheiden ist. Dabei wird eine Normalmode stets mit derselben Nummer versehen, auch wenn sie durch Deuterierung stark verschoben wird. Die Fundamentalübergänge des Ameisensäuremonomers und seiner deuterierten Isotopologen wurden unter anderen in Referenz 16 in Jetexpansionen untersucht und mit den dort aufgeführten früheren spektroskopischen Arbeiten verglichen. 


\section{Ameisensäuredimer}

Die in diesem Kapitel durchgeführte genaue Bestimmung der Bandenzentren verschiedener Isotopologe des Ameisensäuredimers im Bereich von 200 bis $1850 \mathrm{~cm}^{-1}$ sollte sich für die Interpretation des höheren Spektralbereichs oberhalb von $2000 \mathrm{~cm}^{-1}$ als nützlich erweisen, in dem zahlreiche Kombinationen und Obertöne zu beobachten sind. ${ }^{16,17,246-248}$ Zusätzlich ist die genaue Kenntnis der Bandenzentren wichtig für eine experimentelle Abschätzung der Nullpunktsenergie des Ameisensäuredimers und für Bezugswerte für quantenchemische Schwingungsrechnungen. Für alle hier untersuchten Banden können deutliche thermische Verschiebungen in der warmen Gasphase festgestellt werden, sodass die Untersuchung in Jetexpansionen sich als vorteilhaft erweist. Auch sollte die Untersuchung von größeren Clustern als dem Dimer möglich sein, da Banden größerer Spezies in den Spektren des Filet-Jet nachgewiesen werden können. Möglicherweise lassen sich auch spektroskopische Hinweise auf acyclische Dimere der Ameisensäure finden. ${ }^{249-253}$

\subsection{Messbedingungen}

Tabelle 6.1.: Experimentelle Bedingungen der in den Expansionen des Filet-Jet aufgenommenen Ameisensäurespektren: Stagnationsdruck $p_{\mathrm{st}}$, Sättigertemperaturen $T_{\mathrm{S}}$ und Anteil des Gases aus einem Sättiger an der Gesamtmischung im Reservoir nach Zumischung von Helium über eine weitere Zuleitung oder bei der Mischung von Wasser mit $\mathrm{HCOOH}$ aus zwei unterschiedlichen Sättigern. Die Konzentrationen der Substanzen im Reservoir wurde aus ihren Gleichgewichtsdampfdrücken bei der Sättigertemperatur und einem Heliumdruck von 1.6 bar im Sättiger abgeschätzt.

\begin{tabular}{lcrcc}
\hline & $p_{\text {st }} /$ bar & $T_{\mathrm{S}} /{ }^{\circ} \mathrm{C}$ & Verdünnung & Konzentration \\
\hline $\mathrm{HCOOH}$ & 0.7 & -10 & $100 \%$ & $0.4 \%$ \\
& 0.5 & 10 & $20 \%$ & $0.2 \%$ \\
& 0.3 & 10 & $5 \%$ & $0.1 \%$ \\
$\mathrm{HCOOH}$ & 0.4 & 10 & $10 \%$ & $0.1 \%$ \\
$+\mathrm{H}_{2} \mathrm{O}$ & 0.7 & -20 & $90 \%$ & $0.1 \%$ \\
Ameisensäureisotopologe & 0.7 & 10 & $10 \%$ & $0.1 \%$ \\
& 0.3 & -10 & $100 \%$ & $0.4 \%$ \\
& & 10 & $10 \%$ & $0.1 \%$ \\
\hline
\end{tabular}

Die Ferninfrarotspektren der Überschallexpansionen von Ameisensäure wurden mit Hilfe eines Bolometers (4 K, „Bolometer_neu“) mit einem internen Bandpassfilter $\left(200-690 \mathrm{~cm}^{-1}\right)$ aufgenommen. Als Optiken wurden CsI Fenster und ein $6 \mu$ Mylar Strahlteiler verwendet. Auf Grund der Transmissionslücke des Strahlteilers zwischen 370 und $390 \mathrm{~cm}^{-1}$, wird dieser Wellenzahlbereich in den Ferninfrarotspektren nicht abgebildet. Die Spektren wurden mit einer Apertur von $5 \mathrm{~mm}$ und bei Auflösungen von $4 \mathrm{~cm}^{-1}$ (20 kHz Spiegelgeschwindigkeit), $2 \mathrm{~cm}^{-1}(40 \mathrm{kHz})$ und $1 \mathrm{~cm}^{-1}(40 \mathrm{kHz})$ aufgenommen. Als Lichtquelle wurde der Globar des Spektrometers verwendet. 
Die Spektren im mittleren Infrarotbereich von 600 bis $1850 \mathrm{~cm}^{-1}$ wurden mit dem MCTElement eines InSb/MCT-Sandwichdetektors (77 K, „Sandwich_alt“") aufgenommen. Das InSbElement vor dem MCT wirkt dabei als kalter Filter der oberhalb von etwa $2000 \mathrm{~cm}^{-1}$ undurchlässig ist. Als Optiken wurden $\mathrm{KBr}$ Fenster, Linsen und ein $\mathrm{KBr}$ Strahlteiler verwendet. Als Apertur wurden $5 \mathrm{~mm}$ gewählt und die Spektren bei Auflösungen von 2, 1 und $0.5 \mathrm{~cm}^{-1}$ mit einer Spiegelgeschwindigkeit von $80 \mathrm{kHz}$ aufgenommen. Die Bedingungen, unter denen die Gasgemische im Reservoir hergestellt wurden, sind in Tabelle 6.1 zusammengefasst.

\section{2. $(\mathrm{HCOOH})_{2}$}

Die Fundamentalbanden des Ameisensäuredimers bei Raumtemperatur sind größtenteils bekannt. ${ }^{17,254,255}$ Ihre Untersuchung bei Jetkühlung kann dazu dienen die Bandenzentren genauer zu bestimmen, ${ }^{16,17,243,247}$ da die thermische Anregung des Dimers verringert wird und sich dadurch die Bandenstrukturen deutlich vereinfachen.

Vor allem das Bandenzentrum der IR-aktiven antisymmetrischen Dimerknickschwingung innerhalb der Molekülebene $\nu_{24}$ lässt sich bei Raumtemperatur nicht leicht bestimmen, da die thermische Anregung zu einer sehr breiten Bande dieses Übergangs führt. In der Literatur liegen die angegebenen Werte aus Raumtemperaturdaten für diese intermolekulare Mode zwischen 237 und $268 \mathrm{~cm}^{-1} \cdot{ }^{17,254,256-258}$ Erst durch die Kühlung in Jetexpansionen konnte das Bandenzentrum dieser intermolekularen Schwingung bei $264 \mathrm{~cm}^{-1}$ festgelegt werden. ${ }^{15}$ Dadurch wurde ein wichtiger Unsicherheitsfaktor bei der Bestimmung der Zustandssummen des Ameisensäuredimers zwischen $0 \mathrm{~K}$ bis $296 \mathrm{~K}$ beseitigt, was wiederum die Bestimmung der Dissoziationsenergie $D_{0}$ aus experimentellen Werten für die Gleichgewichtskonstanten $K_{p}$ zwischen Monomer und Dimer mit hoher Genauigkeit erlaubte. ${ }^{15}$

Die Ferninfrarotspektren von Ameisensäure sind in Abbildung $6.2 \mathrm{im}$ Bereich von 200 bis $675 \mathrm{~cm}^{-1}$ abgebildet. Im Jetspektrum c können die Rotationszweige der Bande von $\nu_{24}$ aufgelöst werden. Der $P$ Zweig zeigt sich als schwache Schulter bei $262.8 \mathrm{~cm}^{-1}$ und die klar erkennbaren $Q$ und $R$ Zweige bei 264.0 und $265.2 \mathrm{~cm}^{-1}$. Diese Beobachtung passt zu der Erwartung einer $A$ Typ Bande (Vgl. Tabelle 6.2) und einer starken Rotationskühlung des Dimers. Außerdem offenbart sich bei hoher Auflösung ein mit C gekennzeichnetes Signal bei $270 \mathrm{~cm}^{-1}$, das vermutlich auf größere Cluster oder eine heiße Bande zurückzuführen ist. Die fehlende Rotationsstruktur von $\mathrm{C}$ und der hohe Stagnationsdruck, sowie die hohe Konzentration der Ameisensäure in den Jetexpansionen sprechen eher für die erstere Zuordnung. Durch die geringere Auflösung zeigt das Jetspektrum b ein deutlich verbessertes Signal-zu-Rauschen Verhältnis, das die Beobachtung einiger Kombinationsbanden von intermolekularen Moden ${ }^{17,243}$ ermöglicht. Das Signal bei $311 \mathrm{~cm}^{-1}$ ist die Kombinationsbande aus $\nu_{16}{ }^{17}$ und $\nu_{12}{ }^{243}\left(69 \mathrm{~cm}^{-1}+242 \mathrm{~cm}^{-1}=311 \mathrm{~cm}^{-1}\right),{ }^{17,243}$ bei $419 \mathrm{~cm}^{-1}$ liegt $\nu_{24}+\nu_{9}{ }^{243}\left(264 \mathrm{~cm}^{-1}+161 \mathrm{~cm}^{-1}=425 \mathrm{~cm}^{-1}\right)$ und bei $451 \mathrm{~cm}^{-1}$ kann $\nu_{24}+$ $\nu_{8}^{243}\left(264 \mathrm{~cm}^{-1}+194 \mathrm{~cm}^{-1}=458 \mathrm{~cm}^{-1}\right)$ zugeordnet werden. Tatsächlich entsprechen diese drei Kombinationsbanden, die alle $B_{u}$ Symmetrie aufweisen, den intensivsten vorhergesagten IRaktiven Kombinationstönen, die in der anharmonischen Schwingungsrechnung auf B3LYP-D3/6$311+G(2 d, p)-N i v e a u$ vorhergesagt werden (Vgl. Tabelle 6.2). Alle anderen IR-aktiven Kombinationstöne werden im Bereich von 200 bis $675 \mathrm{~cm}^{-1}$ um mindestens einen Faktor 5 schwä- 


\section{Ameisensäuredimer}

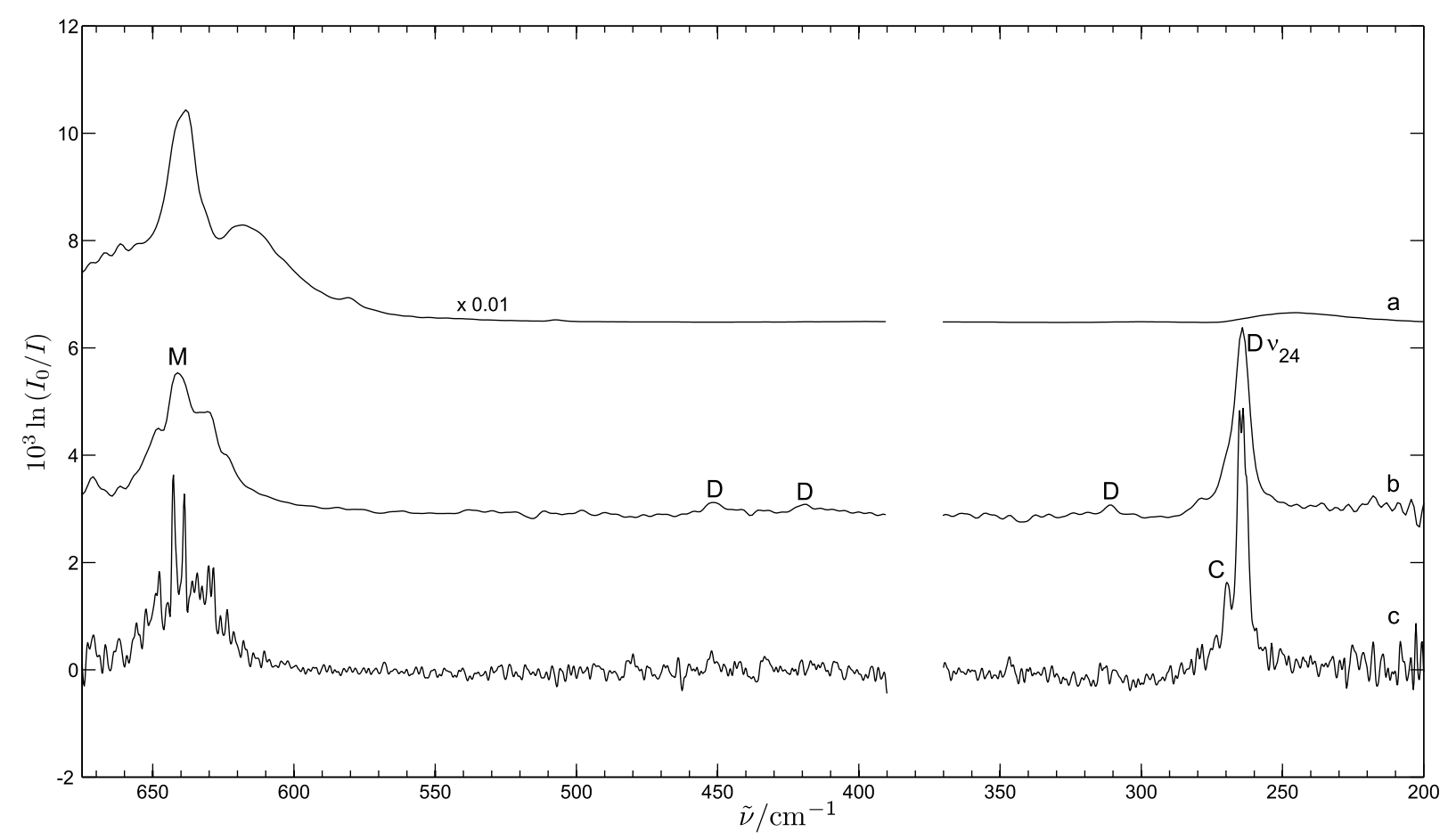

Abbildung 6.2.: Spektren von Ameisensäure im Ferninfrarotbereich: a - Gasphase bei Raumtemperatur und Jetspektren von $0.4 \%$ Ameisensäure in 0.7 bar Helium bei b $-4 \mathrm{~cm}^{-1}$ Auflösung (250 Pulse) und c - $1 \mathrm{~cm}^{-1}$ Auflösung (400 Pulse). Gekennzeichnet sind die Banden des Monomers (M), des Dimers (D) und größerer Cluster (C).

cher vorhergesagt. Die berechneten außerdiagonalen Anharmonizitäten von $\mathrm{x}_{16,12}=+0.2 \mathrm{~cm}^{-1}$, $\mathrm{x}_{24,9}=-3.8 \mathrm{~cm}^{-1}$ und $\mathrm{x}_{24,8}=-7.8 \mathrm{~cm}^{-1}$ passen gut zu den jeweiligen experimentellen Werten von $0,-6$ und $-7 \mathrm{~cm}^{-1}$.

Die IR-aktive intramolekulare Fundamentale bei niedrigster Wellenzahl entspricht der antisymmetrischen $\mathrm{C}-\mathrm{C}-\mathrm{O}$ Knickschwingung $\nu_{23}$ der Schweratome des Ameisensäuredimers. Sie wurde in der Gasphase bisher bei 695 bis $699 \mathrm{~cm}^{-1}$ verortet. ${ }^{17,254,255}$ In Abbildung 6.3 ist an dieser Position kein Dimersignal zu finden. Stattdessen zeigen sich zwei Banden bei $709 \mathrm{~cm}^{-1}$ und $717 \mathrm{~cm}^{-1}$. Die Interpretation dieses Bandensystems ist auf Grund der geringen Intensität schwierig. Zum einen könnten beide Banden auf das Ameisensäuredimer zurückgehen, möglich wäre zum Beispiel eine Fermiresonanz. Dafür spricht, dass beide Banden im hochaufgelösten Spektrum $\mathrm{f}$ in Abbildung 6.3 eine ähnliche Aufspaltung der Rotationsstruktur zeigen mit intensiven Peaks bei 707.6 und $708.8 \mathrm{~cm}^{-1}$ bzw. 716.3 und $717.5 \mathrm{~cm}^{-1}$. Jedoch lässt sich kein passender Übergang mit $B_{u}$ Symmetrie finden, der diese Störung hervorrufen könnte. Zum anderen könnte es sich bei dem Signal um $717 \mathrm{~cm}^{-1}$ auch um eine heiße Bande handeln. Es besteht aber auch die Möglichkeit, dass es sich um ein Signal größerer Cluster handelt. Die Untersuchung des Konzentrationsverhaltens der Bande bei $717 \mathrm{~cm}^{-1}$ (Spektren b und $\mathrm{c}$ in Abbildung 6.3) weist aber eher auf eine Dimerbande als auf ein Signal größerer Cluster hin. Außerdem ist in Abbildung 6.3 ein Spektrum von Jetexpansionen gezeigt bei denen zusätzlich Wasser zugemischt wurde. Auch 


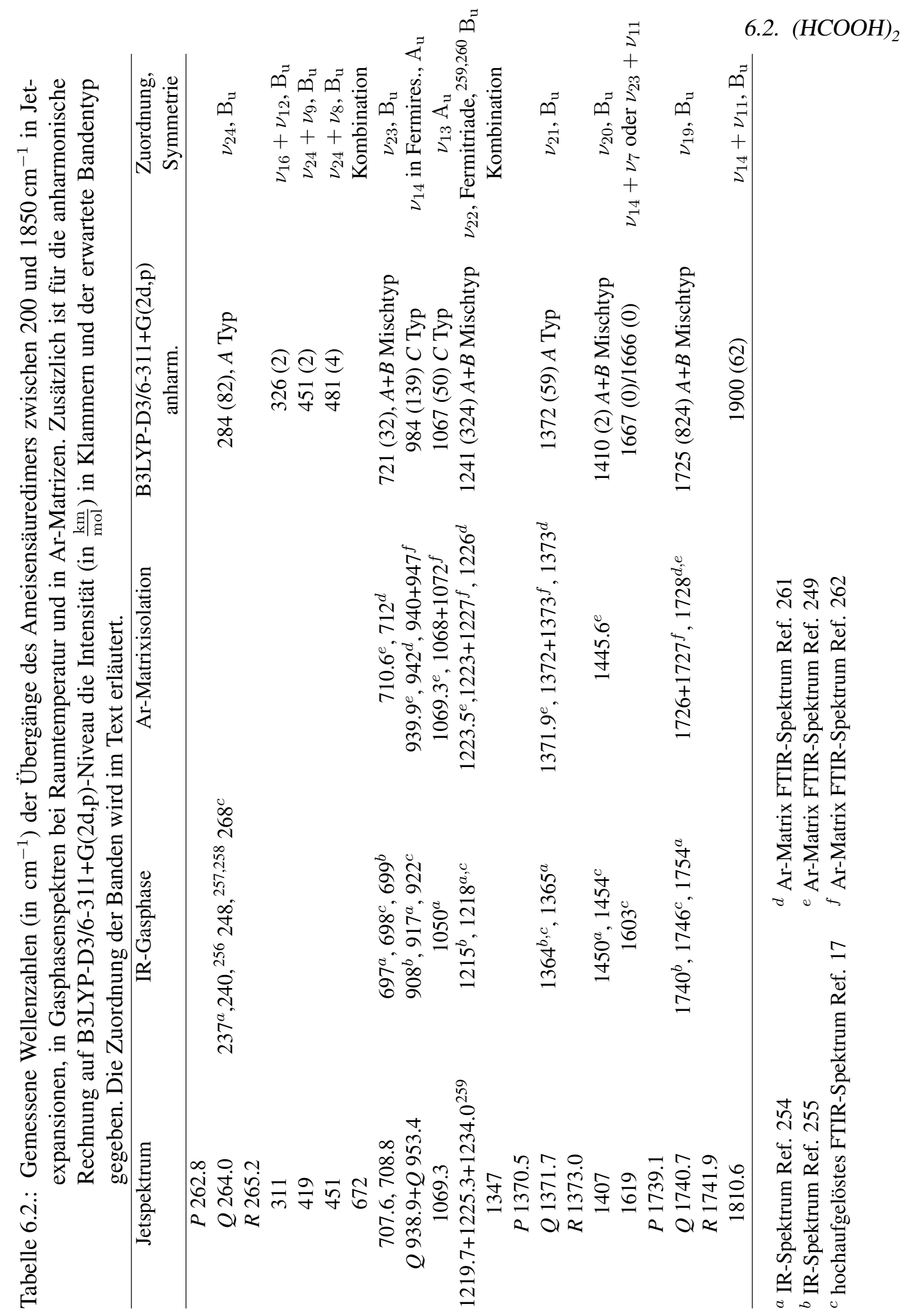




\section{Ameisensäuredimer}

hier ist die Interpretation auf Grund der schwachen Signale schwierig, jedoch scheint die Bande bei $717 \mathrm{~cm}^{-1}$ in diesem Spektrum zu verschwinden. Dies deutet darauf hin, dass sie nicht auf das Ameisensäuredimer zurückgeht. Ein schwaches Dimersignal ist bei $672 \mathrm{~cm}^{-1} \mathrm{zu}$ finden, bei dem es sich möglicherweise um eine Kombinationsbande handelt, die auf der Anregung von mindestens drei intermolekularen Schwingungsquanten beruht.

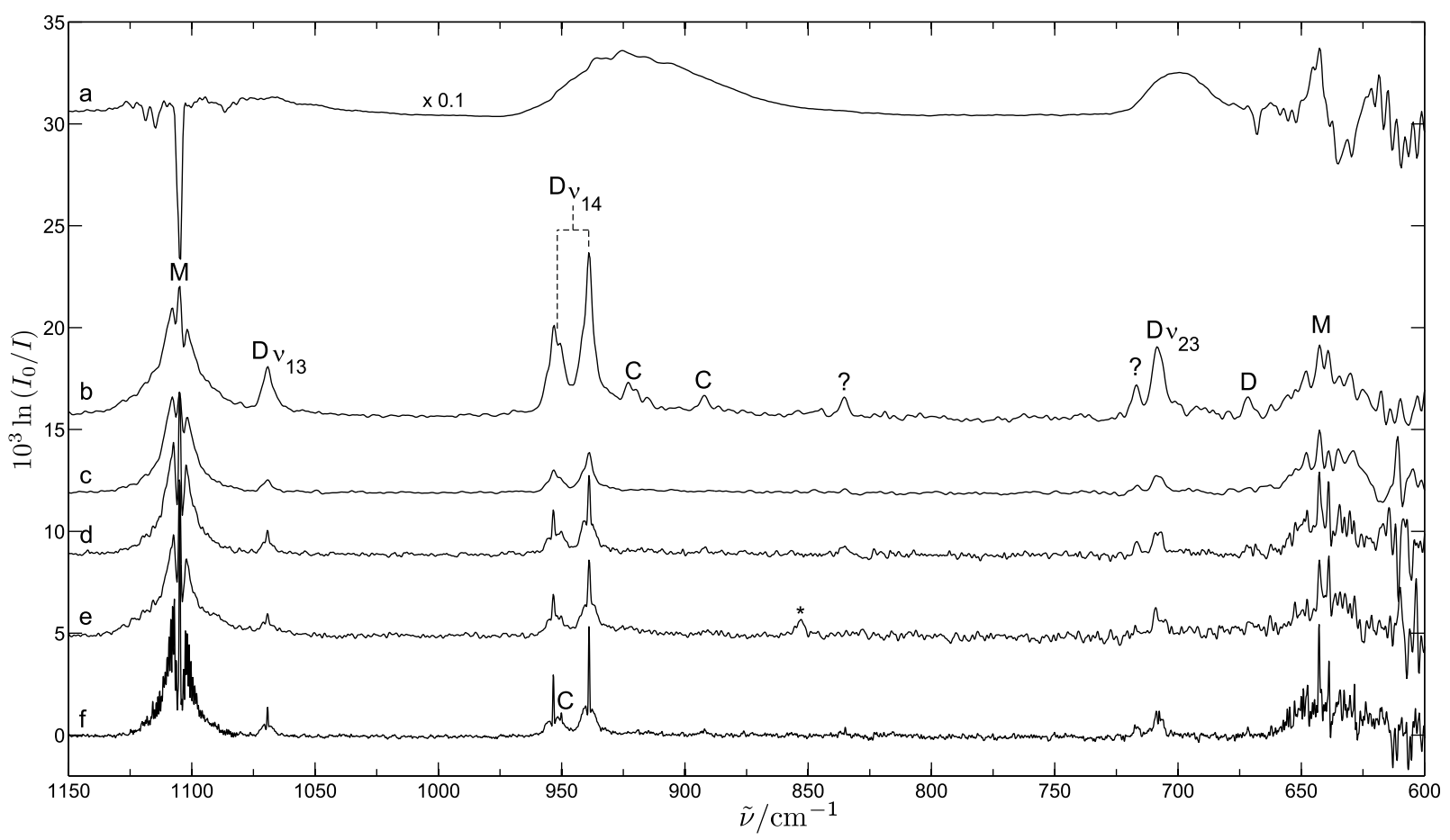

Abbildung 6.3.: Spektren von Ameisensäure im Bereich von 600 bis $1150 \mathrm{~cm}^{-1}$ : a - Differenz von Gasphasenspektren bei Raumtemperatur, die minimale Beiträge des Monomers zeigt, und Jetspektren von Ameisensäure in Helium. b - 0.2\% Ameisensäure in 0.5 bar Helium $\left(2 \mathrm{~cm}^{-1}\right.$ Auflösung, 100 Pulse $)$, c - 0.1\% Ameisensäure in 0.3 bar Helium $\left(2 \mathrm{~cm}^{-1}\right.$ Auflösung, 100 Pulse $), \mathrm{d}-0.1 \%$ Ameisensäure in $0.4 \mathrm{bar}$ Helium ( $1 \mathrm{~cm}^{-1}$ Auflösung, 50 Pulse), e - 0.1\% Ameisensäure und 0.1\% Wasser in 0.7 bar Helium $\left(1 \mathrm{~cm}^{-1}\right.$ Auflösung, 50 Pulse) und $\mathrm{f}-0.1 \%$ Ameisensäure in 0.4 bar Helium $\left(0.5 \mathrm{~cm}^{-1}\right.$ Auflösung, 200 Pulse). Gekennzeichnet sind die Banden des Monomers (M), Dimers (D) und größerer Cluster (C) der Ameisensäure, sowie Banden von gemischten Clustern $\left(^{*}\right)$ und Banden mit unklarer Zuordnung (?).

Die Fundamentale der gleichgerichteten Libration der $\mathrm{O}-\mathrm{H}$ Gruppen $\nu_{14}$ mit $\mathrm{A}_{\mathrm{u}}$ Symmetrie wurde in IR-Spektren bei Raumtemperatur bei 908 bis $922 \mathrm{~cm}^{-1}$ beobachtet. In den Jetspektren in Abbildung 6.3 zeigen sich in der Nähe zwei ähnlich intensive Banden bei 938.9 und $953.4 \mathrm{~cm}^{-1}$. Die Rotationsstruktur offenbart sie im hoch aufgelösten Spektrum $\mathrm{f}$ als $C$ Typ Banden, was zu der Erwartung aus der Schwingungsvorhersage passt (s. Tabelle 6.2). Die Intensitäten beider Banden weisen die Konzentrationsabhängigkeit des Dimers auf. Die Bande bei 
höherer Wellenzahl wird aber durch ein Signal von größeren Clustern bei $950 \mathrm{~cm}^{-1}$ überlagert. Die schlüssigste Interpretation dieses Dimerdubletts ist eine Fermiresonanz. Dafür liegt nahe, dass eine der beiden tieferliegenden intramolekularen Moden $\nu_{7}{ }^{16}$ oder $\nu_{23}$ beteiligt ist, da sonst Kombinationen aus mehr als zwei Schwingungsquanten als störende Niveaus notwendig wären. Die Kombination aus $\nu_{7}$ und $\nu_{24}\left(682 \mathrm{~cm}^{-1}+264 \mathrm{~cm}^{-1}=946 \mathrm{~cm}^{-1}\right)$ hätte eine passende Wellenzahl, aber mit $\mathrm{B}_{\mathrm{u}}$ die falsche Symmetrie. Stattdessen bietet sich die Kombination von $\nu_{23}$ und $\nu_{12}{ }^{243}\left(708 \mathrm{~cm}^{-1}+242 \mathrm{~cm}^{-1}=950 \mathrm{~cm}^{-1}\right)$ mit $\mathrm{A}_{\mathrm{u}}$ Symmetrie an. Aus dem Intensitätsverhältnis von etwa 1:2 ergeben sich entstörte Bandenpositionen von $942 \mathrm{~cm}^{-1}$ für den Fundamentalübergang $\nu_{14}$ und $947 \mathrm{~cm}^{-1}$ für die Kombinationsbande. Aus letzterer lässt sich ein anharmonischer Kreuzterm von ungefähr $-3 \mathrm{~cm}^{-1}$ ableiten, was mit der vorhergesagten Anharmonizität von $\mathrm{x}_{23,12}=-4.1 \mathrm{~cm}^{-1}$ gut übereinstimmt. Eine Fermiresonanz wird von der anharmonischen Analyse nicht explizit prognostiziert, da der energetische Abstand der zwei beteiligten Niveaus zu hoch ist. Wird die Rechnung mit leicht erhöhter Massenzahl $A$ der O-gebundenen Wasserstoffe $(A=1.09)$ durchgeführt, verringert sich der energetische Abstand und eine Fermiresonanz wird erkannt. Eine Aufspaltung der Bande von $\nu_{14}$ wurde auch in Argonmatrizen beobachtet, aber als Effekt durch unterschiedliche Matrixtaschen interpretiert. ${ }^{262}$

Bei etwas niedrigeren Wellenzahlen zeigen sich mehrere schwache Signale in den Spektren in Abbildung 6.3, die nicht den Fundamentalübergängen des Dimers zugeordnet werden können. Bei $835 \mathrm{~cm}^{-1}$ liegt ein Signal, dessen Intensität die gleiche Abhängigkeit von Druck und Substanzkonzentration wie das Dimer zeigt, durch Zumischung von Wasser (Spektrum e) aber verschwindet. Somit kann es nicht dem Ameisensäuredimer zugeordnet werden. Bei 892, 915, 920 und $923 \mathrm{~cm}^{-1}$ liegen Signale, die sich auf Grund ihres Wachstumsverhaltens als Banden von größeren Clustern identifizieren lassen (Spektren b und c). Durch die Zumischung von Wasser in die Expansionen von Ameisensäure (Spektrum e) ergibt sich bei $853 \mathrm{~cm}^{-1}$ ein neues Signal, das in den Spektren der reinen Substanzen nicht zu finden ist. Somit ist die Zuordnung zu einem gemischten Cluster naheliegend.

Bei $1069.3 \mathrm{~cm}^{-1}$ zeigt sich in den Jetspektren in Abbildung 6.3 eine weitere $C$ Typ Bande, die der gleichgerichteten aus der Dimerebene führenden Knickschwingung der $\mathrm{C}-\mathrm{H}$ Gruppen zugeordnet werden kann. In der Gasphase wurde sie bei $1050 \mathrm{~cm}^{-1}$ beobachtet ${ }^{254}$ und in Argonmatrizen bei 1068 bis $1072 \mathrm{~cm}^{-1} .261,262$

Die antisymmetrische $\mathrm{C}-\mathrm{O}$ Streckschwingung $\nu_{22}$ mit $\mathrm{B}_{\mathrm{u}}$ Symmetrie liegt in einem Spektralbereich, der gut zugänglich für Laserstrahlung ist. ${ }^{259,260,263}$ Deshalb konnte $\nu_{22}$ bereits rotationsund tunnelaufgelöst untersucht werden. ${ }^{259,263}$ Das Bandensystem von $\nu_{22}$ tritt in Form einer Fermi-Triade auf, ${ }^{259,260}$ wird also von zwei Kombinationstönen gestört. Die drei Banden zeigen sich als gemischte $A / B$ Typen, wobei aber der $B$-Anteil deutlich überwiegt. ${ }^{259,260,263}$ Auch in den Jetspektren in Abbildung 6.4 ähneln die drei Signale $B$ Typ Banden. Ihre Bandenzentren konnten durch Fits an die Schwingungs-Rotations-Tunnel-Übergänge bei $1219.71,{ }^{259} 1225.3^{259,263}$ und $1233.95^{259} \mathrm{~cm}^{-1}$ bestimmt werden. Diese stimmen mit den Bandenzentren die sich aus dem Filet-Jetspektrum mit $0.5 \mathrm{~cm}^{-1}$ Auflösung (f) ergeben gut überein: 1219.6, 1225.3 und 1234.0 $\mathrm{cm}^{-1}$. Abweichungen der Wellenzahlen von den Literaturdaten und der Rotationszweige vom $B$ Typ sind unter anderem auf die spektrale Überlappung mit dem Monomerübergang der $\mathrm{O}-\mathrm{H}$ Knickschwingung innerhalb der Molekülebene ${ }^{259}$ zurückzuführen, welcher sich in den FiletJetspektren nicht vermeiden lässt. Die Bande bei höchster Wellenzahl wurde wegen ihrer In- 


\section{Ameisensäuredimer}

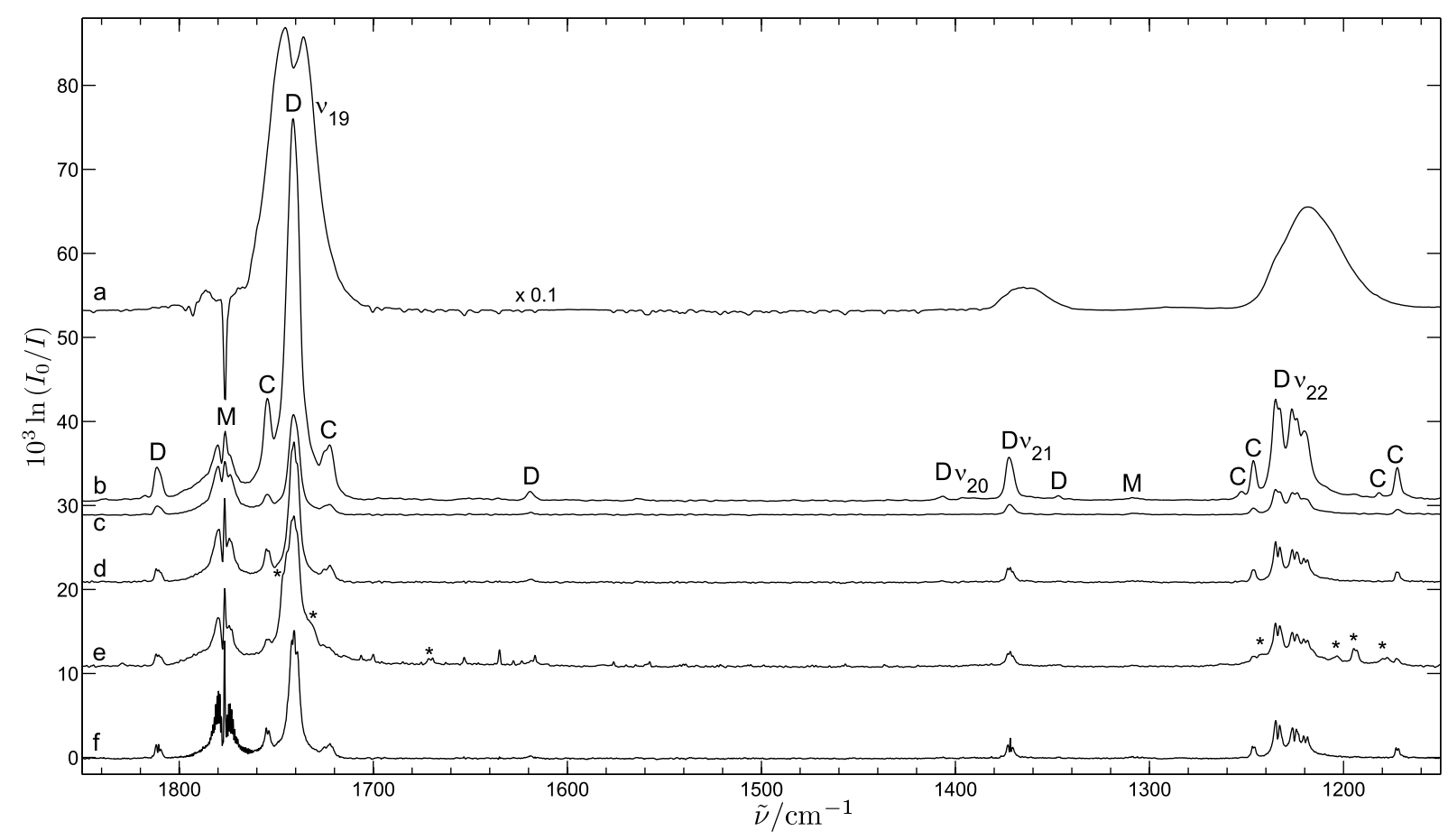

Abbildung 6.4.: Spektren von Ameisensäure im Bereich von 1150 bis $1850 \mathrm{~cm}^{-1}$ : a - Differenz von Gasphasenspektren bei Raumtemperatur, die minimale Beiträge des Monomers zeigt und Jetspektren von Ameisensäure in Helium. b - 0.2\% Ameisensäure in 0.5 bar Helium $\left(2 \mathrm{~cm}^{-1}\right.$ Auflösung, 100 Pulse), c - 0.1\% Ameisensäure in 0.3 bar Helium $\left(2 \mathrm{~cm}^{-1}\right.$ Auflösung, 100 Pulse $), \mathrm{d}-0.1 \%$ Ameisensäure in $0.4 \mathrm{bar}$ Helium ( $1 \mathrm{~cm}^{-1}$ Auflösung, 50 Pulse), e - $0.1 \%$ Ameisensäure und $0.1 \%$ Wasser in 0.7 bar Helium $\left(1 \mathrm{~cm}^{-1}\right.$ Auflösung, 50 Pulse) und $\mathrm{f}-0.1 \%$ Ameisensäure in 0.4 bar Helium $\left(0.5 \mathrm{~cm}^{-1}\right.$ Auflösung, 200 Pulse). Gekennzeichnet sind die Banden des Monomers (M), Dimers (D) und größerer Cluster (C) der Ameisensäure, sowie Banden von gemischten Clustern (*).

tensität und der Konsistenz bei der Entstörung auf den Fundamentalübergang des Dimers zurückgeführt. ${ }^{259,260}$ Die entstörten Bandenpositionen liegen bei $1221,{ }^{259,260} 1228.5^{259} / 1229.6^{260}$ und $1230^{259,260} \mathrm{~cm}^{-1}$. Die störenden Niveaus sollten ebenfalls $B_{u}$ Symmetrie aufweisen. Einer der störenden Übergänge kann auf die Kombination $\nu_{15}{ }^{16}$ und $\nu_{10}{ }^{17}\left(1059 \mathrm{~cm}^{-1}+168.5 \mathrm{~cm}^{-1}=\right.$ $1227.5 \mathrm{~cm}^{-1}$ ) zurückgeführt werden. ${ }^{259,260}$ Dieser wird der mittleren Bande der Fermitriade zugeordnet, ${ }^{259,260}$ woraus sich eine außerdiagonale Anharmonizität von etwa $+1 \mathrm{~cm}^{-1}$ ergibt. In der anharmonischen Rechnung auf B3LYP-D3/6-311+G(2d,p) Niveau wird die Fermiresonanz mit dieser Bande ebenfalls vorhergesagt, für die Anharmonizität $\mathrm{x}_{15,10}=-2.3 \mathrm{~cm}^{-1} \mathrm{der}$ Kombination wird aber keine qualitative Übereinstimmung erreicht. Eine weitere Störbande ergibt sich jedoch nicht in der anharmonischen Analyse. Als weitere Möglichkeit wurde die Kombination aus $\nu_{14}$ und $\nu_{12}{ }^{243}\left(908 \mathrm{~cm}^{-1}+242 \mathrm{~cm}^{-1}=1150 \mathrm{~cm}^{-1}\right)$ in Betracht gezogen; ${ }^{260}$ diese Bande ist aber auf Grund der zu geringen Wellenzahl auszuschließen. ${ }^{16,259}$ Alternativ wurde die Kombi- 
nation von drei Schwingungsquanten mit $2 \cdot \nu_{24}$ und $\nu_{23}\left(2 \cdot 264 \mathrm{~cm}^{-1}+708 \mathrm{~cm}^{-1}=1236 \mathrm{~cm}^{-1}\right)$ vorgeschlagen, die der Bande bei der niedrigsten Wellenzahl entsprechen würde. Mit der entstörten Bandenposition bei $1221 \mathrm{~cm}^{-1259,260}$ und der diagonalen Anharmonizität $\mathrm{x}_{24,24}=-5 \mathrm{~cm}^{-115}$ ergäbe sich eine ungefähre außerdiagonale Anharmonizität von $\mathrm{x}_{24,23}=-2.5 \mathrm{~cm}^{-1}\left(2 \cdot \mathrm{x}_{24,23}=\right.$ $\left.1221 \mathrm{~cm}^{-1}-2 \cdot \nu_{24}-\nu_{23}-2 \cdot \mathrm{x}_{24,24}\right)$. Vorhergesagt wird diese qualitativ übereinstimmend mit $-4.2 \mathrm{~cm}^{-1}$. Die Zuordnung dieser Dreierkombination als eine der Störbanden wäre also schlüssig. Eine alternative Kombination aus drei Schwingungsquanten mit $B_{u}$ Symmetrie nahe 1220 bis $1230 \mathrm{~cm}^{-1}$ lässt sich nicht finden.

In der Nähe der Fermi-Triade von $\nu_{22}$ liegen in den Jetspektren in Abbildung 6.4 mehrere Signale, die auf Grund ihres Wachstumsverhaltens größeren Clustern zugeordnet werden können. Diese sind bei 1172, 1182, 1246 und $1256 \mathrm{~cm}^{-1}$ zu finden. An den Positionen der ersten drei dieser Banden zeigen sich ebenfalls in Ramanspektren von Jetexpansionen schwache Signale. ${ }^{16}$ Die Ramanbande bei $1246 \mathrm{~cm}^{-1}$ wurde einer Kombination mit $\mathrm{A}_{\mathrm{g}}$ Symmetrie zugeordnet. ${ }^{16}$ Die IRSpektren in Abbildung 6.4 deuten jedoch darauf hin, dass es sich dabei nicht um ein Signal des Ameisensäuredimers handelt. Durch die Zumischung von Wasser in die Jetexpansionen lassen sich mehrere Signale beobachten, die nicht in den Spektren der Reinsubstanzexpansionen zu beobachten sind. Diese Banden bei 1178, 1194, 1204 und $1242 \mathrm{~cm}^{-1}$ sollten daher von gemischten Clustern aus Wasser und Ameisensäure stammen.

Bei $1371.7 \mathrm{~cm}^{-1}$ zeigt sich in den Jetspektren in Abbildung 6.4 eine Dimerbande, die bei hoher Auflösung (Spektrum f) als $A$ Typ Bande charakterisiert werden kann. Dabei handelt es sich um den Übergang der in der Dimerebene verlaufenden gleichgerichteten Knickschwingung der C-H Gruppen $\nu_{21}$ mit $\mathrm{B}_{\mathrm{u}}$ Symmetrie. Sie wird in der Gasphase bei Raumtemperatur bei 1364 bis $1365 \mathrm{~cm}^{-1}$ gefunden. ${ }^{17,254,255}$

Zwei schwächere Signale sind nahe der Bande von $\nu_{21} \mathrm{zu}$ finden. Bei $1347 \mathrm{~cm}^{-1}$ liegt eine Bande vor, die wahrscheinlich dem Dimer zuzuordnen ist. Es ergibt sich jedoch keine passende Kombination zweier Schwingungsübergänge, die an dieser Position eine Kombinationsbande zeigen könnten. Eine Fermiresonanz mit dem ersten angeregten Zustand von $\nu_{21}$ ist damit auch unwahrscheinlich. Ein weiteres Signal zeigt sich bei $1407 \mathrm{~cm}^{-1}$. Dabei könnte es sich um eine Kombinationsbande handeln; ein passendes Niveau zweier angeregter Schwingungszustände ergibt sich jedoch auch hier innerhalb von $\pm 15 \mathrm{~cm}^{-1}$ nicht. Es ist möglich, dass es sich bei der Bande um den sehr schwachen Übergang der gleichgerichteten in der Dimerebene verlaufenden Knickschwingung der $\mathrm{O}-\mathrm{H}$ Gruppen $\nu_{20}$ handelt. In der Gasphase bei Raumtemperatur wurde dieser bei 1450 bis $1454 \mathrm{~cm}^{-1}$ verortet, ${ }^{17,254}$ in der Matrix wurde dieser Übergang bei $1446 \mathrm{~cm}^{-1}$ zugeordnet. ${ }^{249}$ Im Bereich um $1450 \mathrm{~cm}^{-1}$ kann jedoch kein Signal in den Expansionsspektren von Ameisensäure beobachtet werden. Möglich ist, dass der Übergang von $\nu_{20} \mathrm{zu}$ schwach ist, um ihn am Filet-Jet zu beobachten. Die vorhergesagte Intensität auf B3LYP-D3/6$311+\mathrm{G}(2 \mathrm{~d}, \mathrm{p})$-Niveau ist mit $2.1 \frac{\mathrm{km}}{\mathrm{mol}}$ (anharmonisch) bzw. $0.3 \frac{\mathrm{km}}{\mathrm{mol}}$ (harmonisch) um mindestens eine Größenordnung kleiner als alle anderen vorhergesagten Intensitäten der IR-aktiven intramolekularen Fundamentalen. Es gibt jedoch Hinweise, die für die Zuordnung von $\nu_{20}$ zum Signal bei $1407 \mathrm{~cm}^{-1}$ sprechen und damit gleichzeitig gegen die Zuordnung in der Literatur bei $1450 \mathrm{~cm}^{-1}$. Der ramanaktive Gegenpart $\nu_{4}$, also die entgegen gerichtete Knickschwingung der $\mathrm{O}-\mathrm{H}$ Gruppen innerhalb der Dimerebene, wurde in Jetexpansionen bei $1431 \mathrm{~cm}^{-1}$ zugeordnet. $^{16}$ In den harmonischen und anharmonischen Rechnungen auf B3LYP-D3/6-311+G(2d,p) sowie 


\section{Ameisensäuredimer}

harmonischen und anharmonischen Rechnungen auf B3LYP ${ }^{16}$ und MP2-Niveau ${ }^{16}$ mit verschiedenen Basissätzen, wird die IR-aktive Mode $\nu_{20}$ konsistent bei niedrigerer Wellenzahl als die ramanaktive Mode $\nu_{4}$ vorhergesagt. Dies spricht gegen die Zuordnung von $\nu_{20}$ bei $1450 \mathrm{~cm}^{-1}$ und für eine Zuordnung unterhalb von $1431 \mathrm{~cm}^{-1}$. In absoluten Werten liegt die vorhergesagte Davydovaufspaltung ${ }^{3,240,264}$ zwischen beiden Moden auf B3LYP-D3/6-311+G(2d,p)-Niveau bei $39 \mathrm{~cm}^{-1}$ (anharmonisch) und $33 \mathrm{~cm}^{-1}$ (harmonisch) und in den harmonischen B3LYP- und MP2Rechnungen ${ }^{16}$ bei 18 bis $32 \mathrm{~cm}^{-1}$ bzw. 9 bis $35 \mathrm{~cm}^{-1}$. Anharmonisch liegen die entsprechenden Werte bei $36 \mathrm{~cm}^{-1}$ (B3LYP) ${ }^{16}$ bzw. 1 bis $29 \mathrm{~cm}^{-1}$ (MP2). ${ }^{16}$ Diese Werte sind größtenteils mit einer experimentellen Aufspaltung von $24 \mathrm{~cm}^{-1}$ konsistent. Die absolute Wellenzahl wird in der anharmonischen Rechnung auf B3LYP-D3/6-311+G(2d,p) bei $1410 \mathrm{~cm}^{-1}$ vorhergesagt, also sehr nah an der experimentell beobachteten Bande bei $1407 \mathrm{~cm}^{-1}$. In Referenz 17 wurde in der Gasphase ein Signal bei $1393 \mathrm{~cm}^{-1}$ beobachtet, bei dem es sich um die tatsächliche Bande von $\nu_{20}$ bei Raumtemperatur handeln könnte. Für das Dimer der C-deuterierten Ameisensäure kann in den Expansionen des Filet-Jet die entsprechende Bande von $\nu_{20}$ bei $1381 \mathrm{~cm}^{-1}$ gefunden werden (siehe Abschnitt 6.3). Damit ergäbe sich für diese Bande eine Isotopenverschiebung von $-26 \mathrm{~cm}^{-1}$, die vergleichbar mit der Isotopenverschiebung der entsprechenden Ramanbande $\nu_{4}$ von $-13 \mathrm{~cm}^{-1}$ ist. ${ }^{16}$ Insgesamt sprechen diese Beobachtungen also deutlich für die Zuordnung von $\nu_{20}$ bei $1407 \mathrm{~cm}^{-1}$.

Im Bereich von 1420 bis $1700 \mathrm{~cm}^{-1}$ zeigt sich in den Jetspektren nur ein einziges schwaches Signal bei $1619 \mathrm{~cm}^{-1}$. Die Zuordnung zu einer Kombinationsbande liegt nahe. In der Nähe dieser Bande liegen drei Kombinationszustände mit ungerader Symmetrie: $\nu_{14}$ und $\nu_{7}^{16}$ $\left(942 \mathrm{~cm}^{-1}+682 \mathrm{~cm}^{-1}=1624 \mathrm{~cm}^{-1}\right), \nu_{23}$ und $\nu_{11}{ }^{16}\left(708 \mathrm{~cm}^{-1}+911 \mathrm{~cm}^{-1}=1619 \mathrm{~cm}^{-1}\right)$ sowie $\nu_{21}$ und $\nu_{12}{ }^{16}\left(1372 \mathrm{~cm}^{-1}+242 \mathrm{~cm}^{-1}=1614 \mathrm{~cm}^{-1}\right)$. Zieht man die vorhergesagten außerdiagonalen Anharmonizitäten von $\mathrm{x}_{14,7}=-1.6 \mathrm{~cm}^{-1}, \mathrm{x}_{23,11}=-5.6 \mathrm{~cm}^{-1}$ und $\mathrm{x}_{21,12}=-0.8 \mathrm{~cm}^{-1}$ in Betracht, so lässt sich letztere Kombination eher ausschließen. Am wahrscheinlichsten erscheint $\nu_{14}+\nu_{7}$, jedoch ist auch $\nu_{23}+\nu_{11}$ möglich. Diese Kombinationsbande entspricht möglicherweise dem Signal, das in Gasphase bei $1603 \mathrm{~cm}^{-1}$ beobachtet wird, ${ }^{17}$ was einer thermischen Verschiebung von $-16 \mathrm{~cm}^{-1}$ entspricht und damit gut vereinbar mit den thermischen Verschiebungen der in Frage kommenden Fundamentalbanden ist.

Die antisymmetrische Carbonylstreckschwingung $\nu_{19}$ mit $\mathrm{B}_{\mathrm{u}}$ Symmetrie zeigt sich in den Jetspektren in Abbildung 6.4 bei $1740.7 \mathrm{~cm}^{-1}$ als sehr intensive Bande, die dem $A$ Typ aber mit schwachem $Q$ Zweig ähnelt. Erwartet wird eine $A / B$ Mischtyp Bande mit überwiegendem $B$ Anteil, der $Q$ Zweig sollte also vom $P$ und $R$ überlagert sein. Benachbart zeigen sich zwei Banden bei 1722 und $1754 \mathrm{~cm}^{-1}$, die größeren Clustern zugeordnet werden können. Im Gasphasenspektrum bei Raumtemperatur (Spektrum a) ähnelt der Übergang von $\nu_{19}$ einer $B$ Typ Bande, deren Minimum genau auf der Position des $Q$ Zweiges dieser Bande im Jetspektrum (Spektrum $\mathrm{f}$ ) liegt. Das deutet darauf hin, dass die antisymmetrische Carbonylstreckschwingung des Dimers durch thermische Anregung bei Raumtemperatur nicht verschoben wird. Außerdem ermöglichen die erkennbaren Rotationszweige des warmen Gasphasenspektrums eine Abschätzung der Rotationstemperatur des Ameisensäuredimers im Jet. Unter der Annahme dass die Wurzel aus der Rotationstemperatur proportional zur Aufspaltung $\Delta$ zwischen den Maxima des $P$ und $R$ Zweiges ist, ergibt sich mit $\Delta_{\mathrm{GP}}=11 \mathrm{~cm}^{-1}$ und $\Delta_{\text {Jet }}=2.6(2) \mathrm{cm}^{-1}$ unter den Expansionsbedingungen von Spektrum f eine Rotationstemperatur von 14 bis $19 \mathrm{~K}$ für das Ameisensäuredimer. Für das 


\section{2. $(\mathrm{HCOOH})_{2}$}

Monomer ergeben sich für die Bande der Carbonylstreckschwingung bei $1777 \mathrm{~cm}^{-1}$ Aufspaltungen von $\Delta_{\mathrm{GP}}=28 \mathrm{~cm}^{-1}$ und $\Delta_{\text {Jet }}=5.2(2) \mathrm{cm}^{-1}$ und für die C-O Streckschwingung bei $1105 \mathrm{~cm}^{-1} \Delta_{\mathrm{GP}}=30 \mathrm{~cm}^{-1}$ und $\Delta_{\text {Jet }}=5.1(2) \mathrm{cm}^{-1}$. Daraus lassen sich für das Monomer niedrigere Rotationstemperaturen von 8 bis $11 \mathrm{~K}$ ableiten. Die Rotationstemperatur des Monomers ließe sich noch genauer bestimmen, wenn die Intensitäten der einzeln aufgelösten Rotationslinien dieser Banden ausgewertet würden. Besonders auf Grund der groben Näherung der quadratischen Abhängigkeit der Aufspaltung $\Delta$ von der Rotationstemperatur, wäre dies eine gute Kontrolle der hier vorgenommenen Abschätzung.

Ein weiteres Dimersignal zeigt sich bei $1810.6 \mathrm{~cm}^{-1}$ als $A$ Typ Bande. Es handelt sich bei der Bande nicht um einen Fundamentalübergang. Für eine Kombinationsbande weist dieser Übergang jedoch eine relativ hohe Intensität auf. Allerdings sagt auch die anharmonische Rechnung eine sehr intensive Kombinationsbande aus den beiden Librationsschwingungen $\nu_{14}$ und $\nu_{11}{ }^{16}$ (Vgl. Tabelle 6.2) mit einer hohen außerdiagonalen Anharmonizität von $\mathrm{x}_{14,11}=-34 \mathrm{~cm}^{-1}$ vorher. Experimentell ergibt sich aus der Summe beider Übergänge $\left(942 \mathrm{~cm}^{-1}+911 \mathrm{~cm}^{-1}=1853 \mathrm{~cm}^{-1}\right)$ ein Wert von $-42 \mathrm{~cm}^{-1}$.

Tabelle 6.3.: Bandenpositionen (in $\mathrm{cm}^{-1}$ ) von Signalen, die weder dem Ameisensäuremonomer noch dem stabilsten Ameisensäuredimer zugeordnet werden können. Mit? sind ungeklärte Dimerbanden gekennzeichnet mit $\mathrm{C}$ die Banden von größeren Clustern (siehe Text).

\begin{tabular}{ccccc}
\hline $\mathrm{HCOOH}$ & DCOOH & DCOOD & HCOOD & Kennzeichnung \\
\hline 717 & & & $?$ \\
835 & & & $?$ \\
892 & & & $\mathrm{C}$ \\
915 & & & $\mathrm{C}$ \\
920 & & & $\mathrm{C}$ \\
923 & & 1215 & $\mathrm{C}$ \\
1172 & 1193 & \multirow{2}{*}{1208} & & $\mathrm{C}$ \\
1182 & & & 1289 & $\mathrm{C}$ \\
1246 & 1265 & & & $\mathrm{C}$ \\
1256 & & & 1727 & $\mathrm{C}$ \\
1722 & 1701 & 1695 & 1748 & $\mathrm{C}$ \\
1754 & & & $\mathrm{C}$ \\
\hline
\end{tabular}

In Tabelle 6.3 sind Clusterbanden der Ameisensäure und ihrer deuterierten Isotopologen, die in den Abbildungen des folgenden Abschnitts gezeigt sind, zusammengefasst. In den abgebildeten Spektren dieses Kapitels sind die Banden von größeren Clustern mit „C“ gekennzeichnet. Die Zuordnung dieser Banden erfolgte für die undeuterierte Ameisensäure auf Grund der verstärkten Skalierung ihrer Intensität mit Substanzkonzentration und Stagnationsdruck gegenüber den Banden des Ameisensäuredimers. Dabei unterscheiden sie sich aber untereinander ebenfalls teilweise in ihrem Skalierungsverhalten, sodass davon ausgegangen werden kann, dass unterschiedliche Clustergrößen in den Spektren beobachtet werden. Die Zuordnung der Banden zu größeren Clustern der deuterierten Ameisensäureisotopologen erfolgte nicht durch das Skalierungsver- 


\section{Ameisensäuredimer}

halten, sondern durch Analogie ihrer Positionen gegenüber den jeweiligen Dimerbanden. Eine mögliche Interpretation für die Banden größerer Cluster ist das vorliegen eines Ameisensäuretrimers. ${ }^{265}$ Bei einer möglichen Zuordnung des Trimers in den Spektren können die kürzlich veröffentlichten Daten zu Ameisensäuretrimeren in Edelgasmatrizen hilfreich sein. ${ }^{266}$

Außerdem sind in Tabelle 6.3 die Positionen zweier Signale gegeben, die sich auf Grund ihres Wachstumsverhaltens als Dimerbanden charakterisieren lassen. Sie sind in Abbildung 6.3 mit „?" gekennzeichnet. Da sie bei der Zumischung von Wasser aber im Gegensatz zu den anderen Dimerbanden verschwinden, ist ohne Weiteres eine Zuordnung zum hier untersuchten stabilsten Dimer der Ameisensäure nicht schlüssig. Stattdessen besteht die Möglichkeit, dass sie auf ein anderes Konformer ${ }^{249-253}$ des Ameisensäuredimers zurückgehen. Diese verfügen im Gegensatz zum stabilsten Dimer über freie O-H Bindungen, an die Wasser koordiniert werden kann. Dies würde ihr abweichendes Intensitätsverhalten bei Zumischung von Wasser erklären. Sowohl die Signale der größeren Cluster als auch die ungeklärten Dimerbanden bedürfen genauerer Untersuchung, um die jeweils zu Grunde liegenden Clusterspezies aufzuklären.

Tabelle 6.4.: Gemessene Wellenzahlen (in $\mathrm{cm}^{-1}$ ) der Übergänge von gemischten AmeisensäureWasser Clustern zwischen 600 und $1850 \mathrm{~cm}^{-1}$ in Jetexpansionen und in ArMatrizen. Zusätzlich ist für die anharmonische Dimerberechnung auf B3LYP-D3/6$311+\mathrm{G}(2 \mathrm{~d}, \mathrm{p})-$ Niveau die Intensität (in $\left.\frac{\mathrm{km}}{\mathrm{mol}}\right)$ in Klammern gegeben.

\begin{tabular}{|c|c|c|c|}
\hline Jetspektrum & Ar-Matrixisolation & $\begin{array}{l}\text { B3LYP-D3/6-311+G(2d,p) } \\
\text { anharm. }\end{array}$ & Zuordnung \\
\hline$\overline{853}$ & $829^{b}$ & $866(126)$ & Dimer, $\mathrm{CO}-\mathrm{H} \cdots \mathrm{OH}_{2}$ Libration \\
\hline 1178 & & & größer als Dimer \\
\hline 1194 & $1172.3^{a}, 1173^{b}$ & $1175(230)$ & Dimer, $\mathrm{C}-\mathrm{O}$ Streckschwingung \\
\hline 1204 & & & größer als Dimer \\
\hline 1242 & & & größer als Dimer \\
\hline 1670 & & $1574(96)$ & Dimer, $\mathrm{H}_{2} \mathrm{O}$ Knickschwingung \\
\hline 1732 & & & größer als Dimer \\
\hline 1746 & $1736.7^{a}, 1737^{b}$ & $1734(186)$ & Dimer, $\mathrm{C}=\mathrm{O}$ Streckschwingung \\
\hline
\end{tabular}

${ }^{a}$ Ar-Matrix FTIR-Spektrum Ref. 267

${ }^{b}$ Ar-Matrix FTIR-Spektrum Ref. 268

Durch die Zumischung von Wasser zu den Expansionen von Ameisensäure lassen sich auch im Bereich der Carbonylstreckschwingung zusätzliche Banden beobachten, die nicht in den Spektren der Reinsubstanzen zu beobachten sind. Diese Signale der gemischten Cluster liegen bei 1670, 1732 und $1746 \mathrm{~cm}^{-1}$. In Tabelle 6.4 sind die beobachteten Banden der AmeisensäureWasser Cluster im Bereich von 600 bis $1850 \mathrm{~cm}^{-1}$ zusammengefasst. Durch die Untersuchung der Bandenintensitäten in Abhängigkeit von den Konzentrationen der beiden Substanzen in verschiedenen Expansionsreihen lässt sich eine vorläufige Zuordnung der Banden des gemischten Dimers vornehmen (siehe Tabelle 6.4), welches eine Ringstruktur mit zwei intermolekularen Wasserstoffbrücken aufweist ${ }^{267-269}$ (s. Abbildung 6.1). Beobachtet werden vier Dimerbanden, die sich den vier intensivsten Übergängen der anharmonischen Analyse auf B3LYP-D3/6- 
$311+\mathrm{G}(2 \mathrm{~d}, \mathrm{p})-\mathrm{Niveau}$ in diesem Spektralbereich zuordnen lassen. Dabei handelt es sich um die Libration der O-H Gruppe der Ameisensäure bei $853 \mathrm{~cm}^{-1}$, die C-O Streckschwingung bei $1194 \mathrm{~cm}^{-1}$, die Knickschwingung des Wassers bei $1670 \mathrm{~cm}^{-1}$ und die Carbonylstreckschwingung bei $1746 \mathrm{~cm}^{-1}$. Für die Schwingungen der Ameisensäure stimmen die vorhergesagten Wellenzahlen gut überein, während die Knickschwingung des Wassers um $96 \mathrm{~cm}^{-1}$ tiefer vorhergesagt wird als die experimentell beobachtete Bande. Da die Banden der gemischten Cluster hier experimentell nur am Rande untersucht wurden, ist es empfehlenswert umfassendere Messungen der gemischten Expansionen von Ameisensäure und Wasser durchzuführen, um die hier getroffenen Zuordnungen des gemischten Dimers zu überprüfen und aus den Signalen der größeren Cluster die Stöchiometrie derselben zu bestimmen.

\subsection{Deuterierte Isotopologe des Ameisensäuredimers}

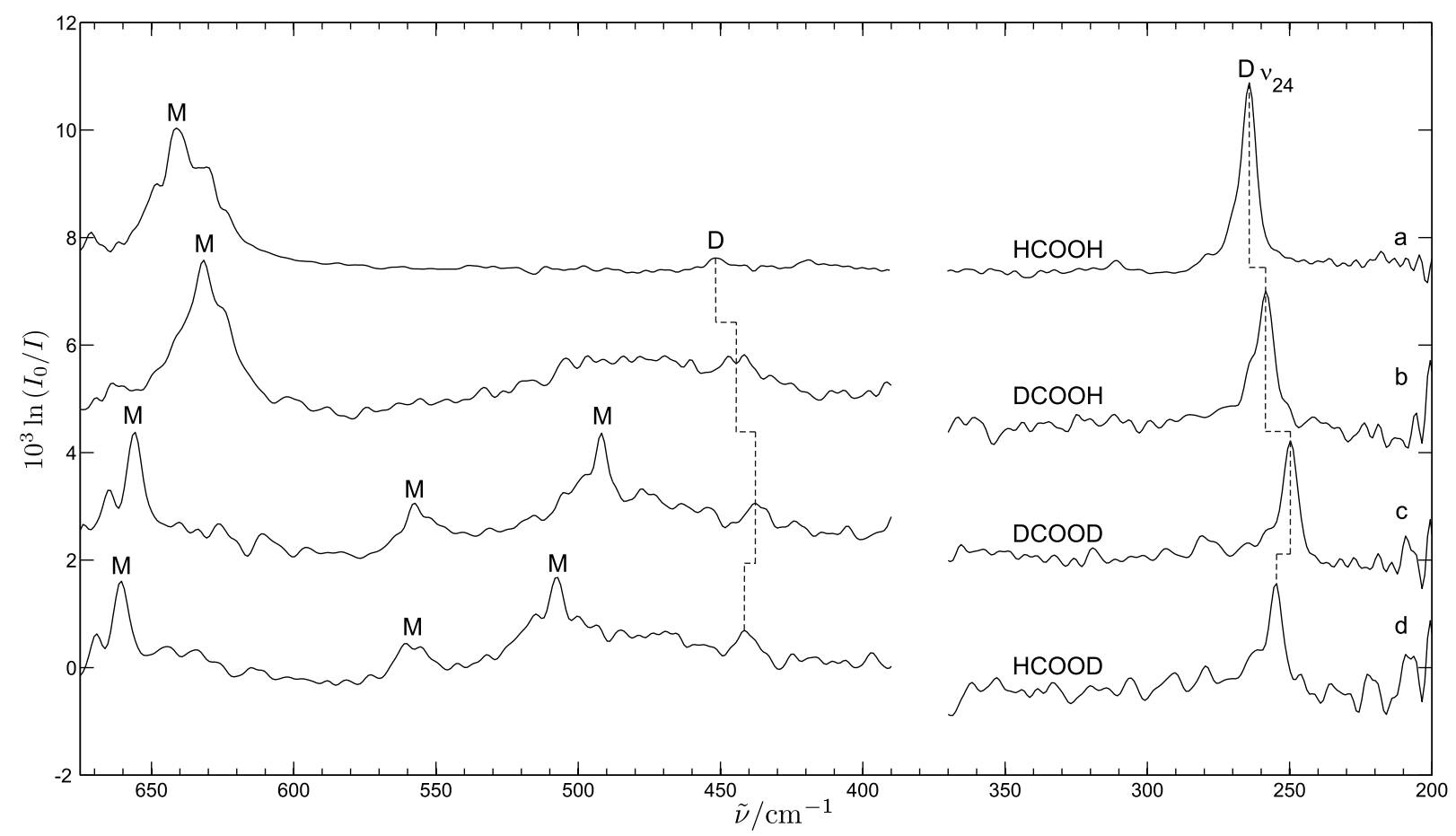

Abbildung 6.5.: Ferninfrarotspektren der Jetexpansionen von 0.4\% Ameisensäure in 0.7 bar Helium: a - HCOOH (250 Pulse), b - DCOOH (20 Pulse), c - DCOOD (20 Pulse) und d - HCOOD (15 Pulse). Gekennzeichnet sind die Banden der Monomere (M) und der Dimere (D). Dimersignale mit gleichartiger Zuordnung sind mit gestrichelten Linien verbunden.

Durch die Expansion von deuterierter Ameisensäure lassen sich am Filet-Jet die symmetrisch deuterierten Isotopologen des Ameisensäuredimers untersuchen. Die tatsächliche Isotopenreinheit der Ameisensäure in den Jetexpansionen lässt sich aus dem Vergleich der Signale der deu- 


\section{Ameisensäuredimer}

terierten Monomere mit den rudimentären Signalen der nicht deuterierten Spezies abschätzen. Sie liegt für DCOOH bei $\geq 95 \%$, für HCOOD bei $\geq 90 \%$ und für DCOOD bei $\geq 99 \%$ C-D und $\geq 95 \%$ O-D. Somit kann davon ausgegangen werden, dass vor allen Dingen die symmetrisch deuterierten Dimere in den Jetexpansionen vorliegen und die unsymmetrisch deuterierten nur einen kleinen Bruchteil der Dimerbevölkerung ausmachen.

In Tabelle 6.5 sind die beobachteten Fundamentalbanden der symmetrisch deuterierten Isotopologen des Ameisensäuredimers zusammengefasst. Die Zuordnung der Dimerbanden basiert auf der Untersuchung der Konzentrationsabhängigkeit ihrer Intensität, der Betrachtung ihrer Rotationsstruktur, sowie auf dem Vergleich mit den Spektren des undeuterierten Dimers und mit der anharmonischen Vorhersage auf B3LYP-D3/6-311+G(2d,p)-Niveau.

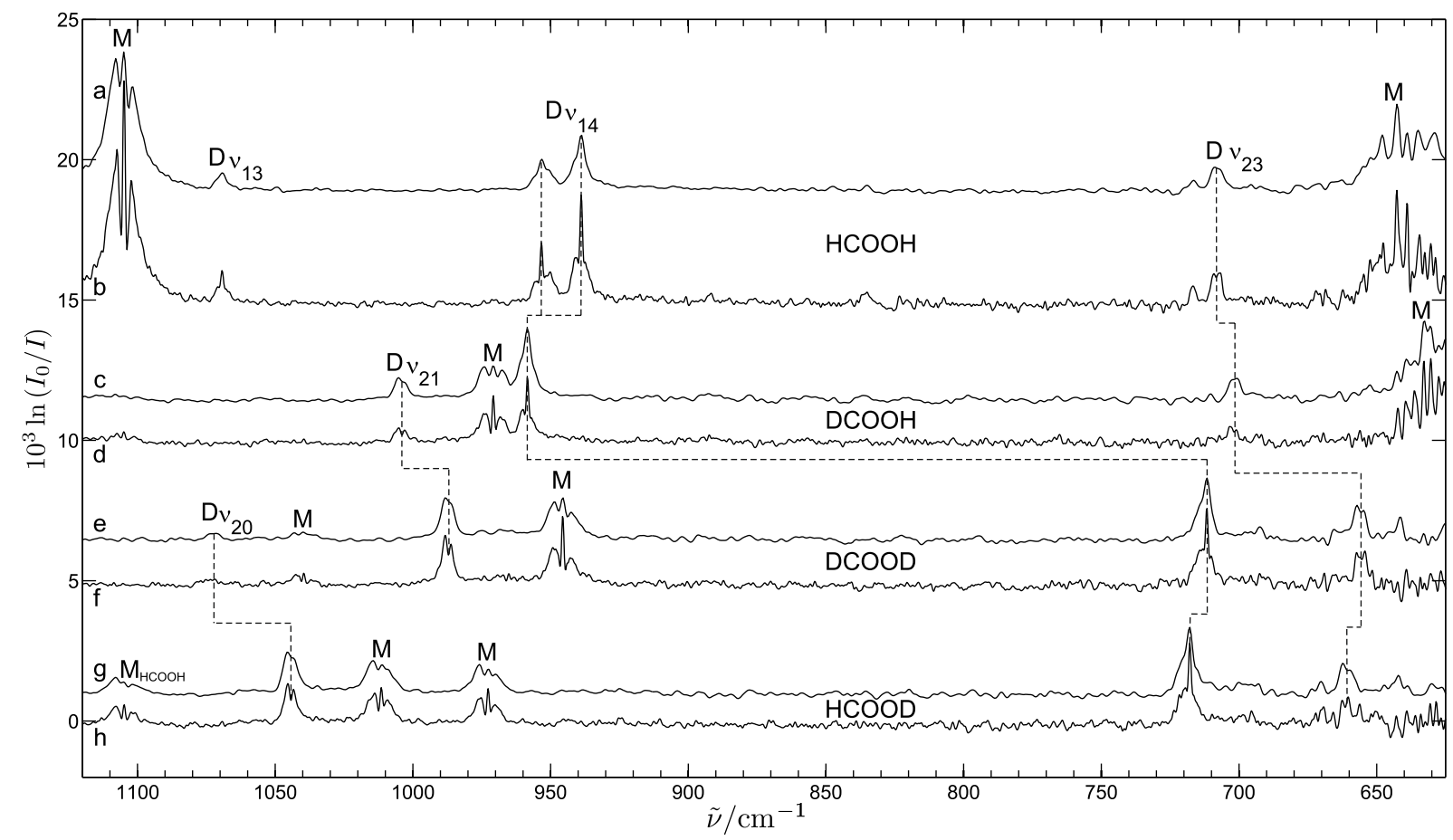

Abbildung 6.6.: Spektren von Ameisensäure im Bereich von 600 bis $1120 \mathrm{~cm}^{-1}$. a $-0.1 \%$ $\mathrm{HCOOH}$ in 0.3 bar Helium $\left(2 \mathrm{~cm}^{-1}\right.$ Auflösung, 50 Pulse $), \mathrm{b}-0.1 \% \mathrm{HCOOH}$ in 0.4 bar Helium $\left(2 \mathrm{~cm}^{-1}\right.$ Auflösung, 50 Pulse), sowie deuterierte Isotopologe: Je $0.1 \%$ in 0.3 bar Helium und 50 Pulse. c,d - DCOOH; e,f - DCOOD und g,h -HCOOD. Die jeweils oberen Spektren a, c, e und g wurden mit $2 \mathrm{~cm}^{-1}$ Auflösung aufgenommen, die jeweils unteren $\mathrm{b}, \mathrm{d}, \mathrm{f}$ und $\mathrm{h}$ mit $1 \mathrm{~cm}^{-1}$ Auflösung. Gekennzeichnet sind die Banden des Monomers (M), Dimers (D) und größerer Cluster $(\mathrm{C})$ der Ameisensäure. Dimersignale mit gleichartiger Zuordnung sind mit gestrichelten Linien verbunden.

Im untersuchten Spektralbereich von 200 bis $1850 \mathrm{~cm}^{-1}$ ergeben sich durch die Deuterierung des Dimers besonders große Verschiebungen für die Knickschwingungen der direkt betroffenen Hydridgruppen. Dabei ändert sich im Falle von $\nu_{21}$ sogar die Bandenform von einer $A$ Typ Bande 
6.3. Deuterierte Isotopologe des Ameisensäuredimers

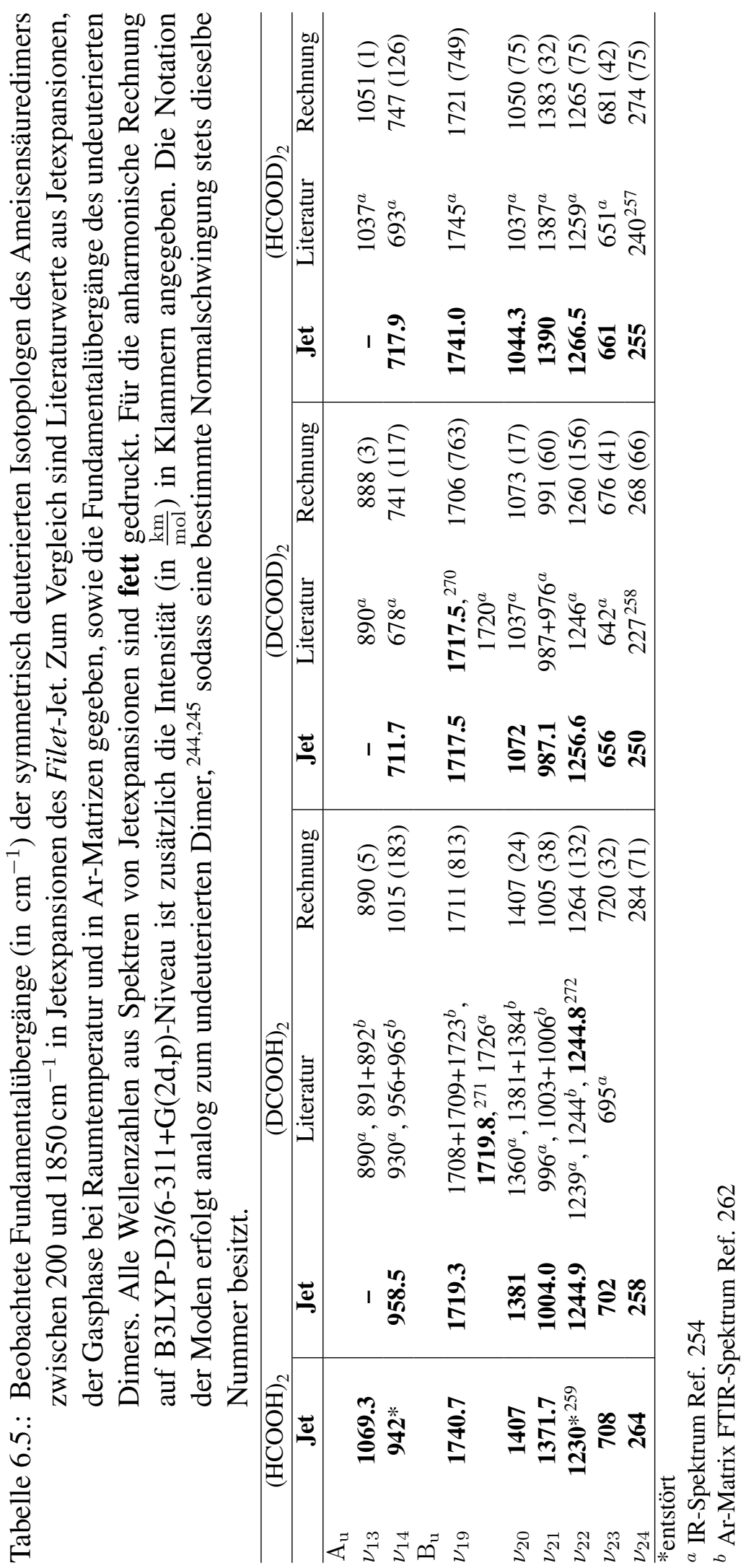




\section{Ameisensäuredimer}

in eine $B$ Typ Bande, wenn der Wasserstoff am Kohlenstoffatom durch Deuterium ersetzt wird (s. Abbildung 6.6). Diese Änderung des Bandentyps wird auch durch die quantenchemische Rechnung vorhergesagt. Da $\nu_{13}$ laut der anharmonischen Vorhersage durch Deuterierung eine Größenordnung an Intensität verliert, ist es einleuchtend, dass die sehr schwachen Übergänge von $\nu_{13}$ für die deuterierten Spezies nicht beobachtet werden. Obwohl beim undeuterierten Dimer der Ameisensäure zwei Fundamentalbanden durch Fermiresonanz als Dublett bzw. Triplett auftreten, zeigen sich bei keinem der symmetrisch deuterierten Dimere im Absorptionsspektrum zwischen 200 und $1850 \mathrm{~cm}^{-1}$ Hinweise auf Fermiresonanzen.

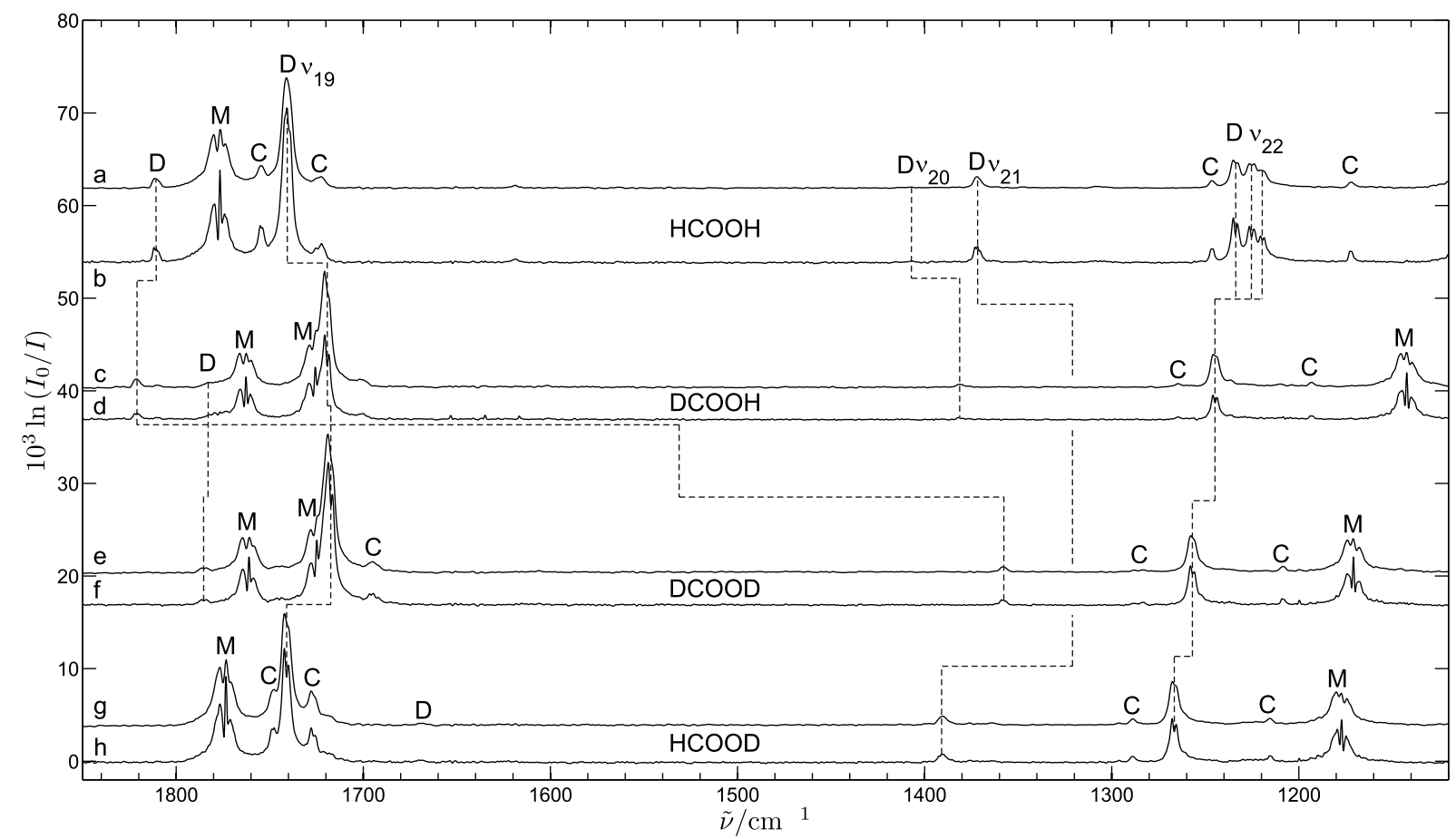

Abbildung 6.7.: Spektren von Ameisensäure im Bereich von 1120 bis $1850 \mathrm{~cm}^{-1}$. a $-0.1 \%$ $\mathrm{CHOOH}$ in 0.3 bar Helium $\left(2 \mathrm{~cm}^{-1}\right.$ Auflösung, 50 Pulse), $\mathrm{b}-0.1 \% \mathrm{HCOOH}$ in 0.4 bar Helium $\left(2 \mathrm{~cm}^{-1}\right.$ Auflösung, 50 Pulse), sowie deuterierte Isotopologe: Je $0.1 \%$ in 0.3 bar Helium und 50 Pulse. c,d - CDOOH; e,f - CDOOD und g,h -CHOOD. Die jeweils oberen Spektren a, c, e und g wurden mit $2 \mathrm{~cm}^{-1}$ Auflösung aufgenommen, die jeweils unteren $\mathrm{b}, \mathrm{d}, \mathrm{f}$ und $\mathrm{h}$ mit $1 \mathrm{~cm}^{-1}$ Auflösung. Gekennzeichnet sind die Banden des Monomers (M), Dimers (D) und größerer Cluster (C) der Ameisensäure. Dimersignale mit gleichartiger Zuordnung sind mit gestrichelten Linien verbunden.

Für die deuterierten Isotopologen können einige Kombinationstöne beobachtet werden, die in Tabelle 6.6 mit den resultierenden außerdiagonalen Anharmonizitäten zusammengefasst sind. Wie auch im undeuterierten Dimer lassen sich die intermolekulare Kombination aus $\nu_{24}$ und $\nu_{8}{ }^{243}$ (s. Abbildung 6.5) und die sehr intensive Kombination aus den beiden Librationsschwingungen der Hydroxygruppen $\nu_{14}$ und $\nu_{11}{ }^{16}$ (s. Abbildung 6.7) zuordnen. Letztere Kombination kann nur 
für (HCOOD $)_{2}$ im Spektrum nicht gefunden werden. Außerdem wird die Kombination von $\nu_{13}$ und $\nu_{10}{ }^{16}$ für $(\mathrm{DCOOH})_{2}$ und (DCOOD) 2 beobachtet, die für ihre Isotopomeren außerhalb des betrachteten Spektralbereichs liegen. Auf Grund der fehlenden Zuordnung der IR-aktiven Fundamentale $\nu_{13}$, kann jedoch keine Anharmonizitätskonstante ermittelt werden. Zusätzlich kann für (HCOOD) $)_{2}$ die Kombination aus $\nu_{20}$ und $\nu_{7}{ }^{16}$ beobachtet werden.

Tabelle 6.6.: Beobachtete Wellenzahlen (in $\mathrm{cm}^{-1}$ ) und außerdiagonale Anharmonizitäten (in $\mathrm{cm}^{-1}$ ) der Kombinationstöne der symmetrisch deuterierten Isotopologen des Ameisensäuredimers. Zum Vergleich sind die experimentellen Banden des undeuterierten Dimers und vorhergesagte Werte aus der anharmonischen Rechnung auf B3LYPD3/6-311+G(2d,p)-Niveau gegeben. Für letztere sind zusätzlich die Intensitäten (in $\left.\frac{\mathrm{km}}{\mathrm{mol}}\right)$ in Klammern angegeben.

\begin{tabular}{|c|c|c|c|c|c|c|c|c|}
\hline & \multicolumn{2}{|c|}{$(\mathrm{HCOOH})_{2}$} & \multicolumn{2}{|c|}{$(\mathrm{DCOOH})_{2}$} & \multicolumn{2}{|c|}{$(\mathrm{DCOOD})_{2}$} & \multicolumn{2}{|c|}{$(\mathrm{HCOOD})_{2}$} \\
\hline & Jet & Rechnung & Jet & Rechnung & Jet & Rechnung & Jet & Rechnung \\
\hline$\overline{\nu_{24}+\nu_{8}}$ & 451 & $481(4)$ & 444 & $484(4)$ & 437 & $463(3)$ & 441 & $470(4)$ \\
\hline $\mathrm{x}_{24,8}$ & -7 & -8 & -7 & -7 & -5 & -7 & -8 & -7 \\
\hline$\nu_{14}+\nu_{11}$ & 1811.6 & $1900(62)$ & 1820.8 & $1913(142)$ & 1357.9 & $1403(51)$ & $\ldots$ & $1411(53)$ \\
\hline $\mathrm{x}_{14,11}$ & -42 & -34 & -59 & -75 & -26 & -37 & ... & -35 \\
\hline$\nu_{20}+\nu_{7}$ & $\ldots$ & 2094 (4) & $\ldots$ & $2088(6)$ & $\ldots$ & $1697(6)$ & 1669 & $1681(26)$ \\
\hline $\mathrm{x}_{20,7}$ & $\ldots$ & -2 & ... & -2 & ... & -2 & -3 & -4 \\
\hline$\nu_{13}+\nu_{10}$ & $\ldots$ & 2109 (9) & 1783 & 1777 (39) & 1785 & 1774 (33) & & $2092(0.1)$ \\
\hline $\mathrm{x}_{13,10}$ & $\ldots$ & -10 & $\ldots$ & -2 & $\ldots$ & -2 & $\ldots$ & -44 \\
\hline
\end{tabular}





\section{Zusammenfassung \& Ausblick}

In dieser Arbeit wurden zahlreiche unterschiedliche Schwingungsmoden in Clustern mit $\mathrm{O}-\mathrm{H}$.. O Wasserstoffbrücken untersucht. Besonderes Augenmerk wurde dabei auf die Schwingungen der Hydroxylgruppen von Wasserstoffbrückendonoren gerichtet.

Durch die Beobachtung der Obertonübergänge der $\mathrm{O}-\mathrm{H}$ Streckschwingungen konnten für Alkoholdimere als einfachste Beispielsysteme für $\mathrm{O}-\mathrm{H} \cdots \mathrm{O}$ Wasserstoffbrücken zwischen organischen Molekülen die anharmonischen Effekte auf diese Schwingungsmoden durch die zwischenmolekulare Wechselwirkung herausgestellt werden. Dies wurde hier erstmals für die im Vakuum isolierten aliphatischen Alkoholdimere durchgeführt, ${ }^{11}$ weshalb die Ergebnisse wichtige Bezugswerte für quantenchemische Rechnungen darstellen. Die beobachtete Zunahme der Anharmonizität der Donorstreckschwingungen und die starke Abschwächung der Obertonintensität gegenüber der Fundamentale sind charakteristische Merkmale bei der Ausbildung von Wasserstoffbrïcken und konnten hier erstmals ohne Umgebungseinflüsse auf die Systeme quantifiziert werden. Die Erweiterung dieser Datenbank für $\mathrm{O}-\mathrm{H}$ Streckschwingungsanharmonizitäten auf weitere Dimere ist wünschenswert. Ein weiteres System ist etwa das gemischte Dimer aus Methanol und Ethylen, dessen Untersuchung gerade publiziert wird und im Zuge der Doktorarbeit von Matthias Heger durchgeführt wurde. ${ }^{273}$ Auch die Untersuchung von Trimerobertönen wäre interessant - wenn auch experimentell sehr herausfordernd.

Die systematische Untersuchung von Librationsschwingungen der $\mathrm{O}-\mathrm{H}$ Gruppe in Clustern konnte hier von den bisher untersuchten Trimeren und Tetrameren von Methanol und tert-Butylalkohol auf ihre Dimere ${ }^{14}$ ausgeweitet werden. Zusätzlich wurden Clusterbanden von Trifluorethanol und Ethanol beobachtet, für Letzteres steht die experimentelle Differenzierung der Librationsbanden zu Clustergrößen noch aus. Bei der Untersuchung der Librationen von Methyllaktatclustern zeigte sich, dass sich aus diesen Schwingungen komplementäre Informationen zu den Erkenntnissen aus dem O-H Streckschwingungsbereich gewinnen lassen. Somit kann der Ferninfrarotbereich bei der Zuordnung von Dimerstrukturen in den Spektren hilfreich sein. Eine Herausforderung ist dabei jedoch die hohe Anharmonizität der Librationen, die für Vergleiche mit Schwingungsvorhersagen von diesen mit betrachtet werden sollte. Da die Librationsschwingungen in einem spektralen Bereich liegen, in dem auch viele Gerüstschwingungen von Molekülen vorkommen ist die Untersuchung größerer Moleküle ohne Größenselektivität zunehmend schwierig, wie die Ferninfrarotspektren von Trifluorethanol zeigen.

Die Infrarotspektren des Ameisensäuredimers und seiner deuterierten Isotopologen konnten im Zuge dieser Arbeit umfassend im Bereich von 200 bis $1900 \mathrm{~cm}^{-1}$ charakterisiert werden. In Messungen der warmen Gasphase erscheinen die meisten Banden des Dimers deutlich verschoben, sodass die hier durchgeführten Messungen in Überschallexpansionen sehr nützlich waren um die tatsächlichen Bandenzentren zahlreicher Übergänge zu ermitteln. Insbesondere die Festlegung der höchstfrequenten intermolekularen Dimerschwingung bei $264 \mathrm{~cm}^{-1}$ hat es er- 


\section{Zusammenfassung \& Ausblick}

möglicht die Zustandssummen des Ameisensäuredimers bis zur Raumtemperatur mit guter Genauigkeit aus experimentellen Daten zu bestimmen. ${ }^{15}$ Mit diesen konnte aus experimentellen Konstanten $K_{p}$ zum Gleichgewicht zwischen Monomer und Dimer in der warmen Gasphase die experimentelle Dissoziationsenergie des Ameisensäuredimers auf einen Wert von 59.5(5) kJ/mol bestimmt werden. ${ }^{15}$ Die umfangreiche Charakterisierung des Ameisensäuredimerspektrums unterhalb von $1900 \mathrm{~cm}^{-1}$ sollte zusammen mit den komplementären Ramandaten ${ }^{16}$ dabei helfen die Spektren oberhalb von $2000 \mathrm{~cm}^{-1}$, die zahlreiche Obertöne und Kombinationsbanden aufweisen, ${ }^{16,17,246-248}$ zuzuordnen.

Bei der Untersuchung von Ethylen- und Propylenglykol konnten im O-H Streckschwingungsbereich die IR-intensivsten Dimerbanden beobachtet werden. Im Falle von Propylenglykol zeigt sich in den Spektren chirale Erkennung. Es scheint eine bevorzugte heterochirale Dimerstruktur in Jetexpansionen vorzuliegen, während für das homochirale Dimer bis zu drei unterschiedliche Geometrien beobachtet wurden. Die Untersuchung der Dimere mittels Ramanspektroskopie sollte die Zuordnung der Strukturen absichern. Diese konnte bisher nur auf Grundlage der berechneten Dissoziationsenergien und Rotverschiebungen ihrer $\mathrm{O}-\mathrm{H}$ Streckschwingung vorgenommen werden. Letztere wurden für Vergleiche mit dem Experiment nur durch harmonischen Schwingungsrechnungen vorhergesagt.

Auch in der Studie zu gemischten Clustern aus Methanol und Aceton konnten skalierte harmonische Wellenzahlen genutzt werden um die Clustergeometrien aufzuklären. ${ }^{9}$ Um den Vergleich zwischen skalierten harmonischen Schwingungsvorhersagen und experimentellen anharmonischen Rotverschiebungen zu rechtfertigen, ist ein besseres Verständnis der Rotverschiebung der O-H Streckschwingung in Wasserstoffbrücken notwendig. Diese Arbeit liefert dafür einen wichtigen Beitrag.

Die Rotverschiebung einer O-H Streckschwingungsfundamentalen in einer Wasserstoffbrücke $\Delta \tilde{\nu}_{\mathrm{OH}}$ entspricht der Differenz der Fundamentalwellenzahlen der freien und der gebundenen Hydroxylgruppe. Sie ergibt sich demzufolge gemäß Formel 2.5 (s. Kapitel 2.1) aus der Änderung der harmonischen Wellenzahl $\Delta \omega_{\mathrm{OH}}$ der O-H Streckschwingung, der Änderung der diagonalen Anharmonizität $\Delta x_{\mathrm{OH}, \mathrm{OH}}$ und der Änderung der außerdiagonalen Kopplungsterme mit den anderen Schwingungen $\Delta x_{\mathrm{OH}, j}$ bei Ausbildung einer Wasserstoffbrücke:

$$
\Delta \tilde{\nu}_{\mathrm{OH}}=\Delta \omega_{\mathrm{OH}}+2 \Delta x_{\mathrm{OH}, \mathrm{OH}}+\frac{1}{2} \sum_{j}^{n} \Delta x_{\mathrm{OH}, j}
$$

Am Beispiel des Methanoldimerdonors lassen sich die Beiträge der einzelnen Einflüsse betrachten. Die experimentelle Verschiebung $\Delta \tilde{\nu}_{\mathrm{OH}}$ beträgt $-110(1) \mathrm{cm}^{-1}$. Die diagonale Anharmonizität wurde in dieser Arbeit bestimmt und steigt vom freien $\mathrm{O}-\mathrm{H}$ im Monomer $\left(x_{\mathrm{OH}, \mathrm{OH}}=\right.$ $\left.-85.8(6) \mathrm{cm}^{-1}\right)$ zum Dimer $\left(x_{\mathrm{OH}, \mathrm{OH}}=-99.2(4) \mathrm{cm}^{-1}\right)$ um 13.4(7) $\mathrm{cm}^{-1}$. Auf die Rotverschiebung des Fundamentalübergangs wirkt sich diese Änderung doppelt aus, somit beträgt der dritte Term in Gleichung 7.1 also $2 \Delta x_{\mathrm{OH}, \mathrm{OH}}=-27(1) \mathrm{cm}^{-1}$. Die harmonische Wellenzahlen sind experimentell nur mit sehr hohem Aufwand zugänglich. Jedoch wurden von Matthias Heger et al. die harmonische Wellenzahlen der $\mathrm{O}-\mathrm{H}$ Streckschwingungen von Methanolmonomer und dimer in einer kürzlich erschienenen Studie durch harmonische Schwingungen auf hohem quantenchemischen Niveau bestimmt. ${ }^{274}$ So konnten sie die harmonische Rotverschiebung des Di- 
merdonors auf $\Delta \omega_{\mathrm{OH}} \approx-121 \mathrm{~cm}^{-1}$ vorhersagen. ${ }^{274}$ Somit kann aus diesen Werten auch der letzte Term in Gleichung 7.1 bestimmt werden und ergibt $\frac{1}{2} \sum_{j}^{n} \Delta x_{\mathrm{OH}, j} \approx+38 \mathrm{~cm}^{-1}$. Es zeigt sich also für den Donor im Methanoldimer, dass sich für die Rotverschiebung der O-H Streckschwingung die Beiträge der diagonalen und außerdiagonalen Anharmonizitäten gegenseitig aufheben und Letztere die diagonalen Anharmonizität sogar überkompensieren. Die anharmonische Rotverschiebung des Dimerdonors ist dadurch kleiner als die harmonische.

Experimentell wäre nun der nächste Schritt, die wichtigsten Beiträge der außerdiagonalen Anharmonizitätskonstanten zur Rotverschiebung des Dimerdonors zu ermitteln. In den hier durchgeführten anharmonischen Schwingungsanalysen zeigt sich, dass der größte Beitrag von der Kopplung mit der Libration kommt. Dies wird ebenfalls aus den Ergebnissen dieser Arbeit verständlich, da gezeigt werden konnte, dass die $\mathrm{O}-\mathrm{H}$ Torsionen durch die Ausbildung von Wasserstoffbrücken besonders starke spektrale Verschiebungen erfahren, was an der Umwandlung nahezu freier Rotationen der $\mathrm{O}-\mathrm{H}$ Gruppe in eingeschränkte Librationsschwingungen liegt. Zudem ist die Kopplung mit der $\mathrm{O}-\mathrm{H}$ Streckschwingung stark, da beide Schwingungen dieselbe funktionelle Gruppe betreffen. Da die Anregung der Libration die Wasserstoffbrücke schwächt erklärt sich auch die resultierende Verminderung der $\mathrm{O}-\mathrm{H}$ Streckschwingungsverschiebung. Nachdem in dieser Arbeit die Fundamentalübergänge der Donorlibration im Methanol- und tertButylalkoholdimer zugeordnet werden konnten, kann nun der Versuch unternommen werden die außerdiagonalen Anharmonizitätskonstanten zwischen $\mathrm{O}-\mathrm{H}$ Streckschwingung und $\mathrm{O}-\mathrm{H}$ Libration aus Kombinationstönen oder heißen Banden zu gewinnen. Weitere wichtige außerdiagonale Beiträge zur Rotverschiebung kommen von der $\mathrm{O}-\mathrm{H}$ Knickschwingung des Donors und den sechs neu entstehenden intermolekularen Schwingungen in Alkoholdimeren. Da die Anregung dieser Schwingungen die Wasserstoffbrücke schwächt, sollten sie zum positiven Wert von $\sum_{j}^{n} \Delta x_{\mathrm{OH}, j}$ beitragen. 



\section{A. Anhang}

\section{A.1. Daten}

Tabelle A.1.: Schwingungsrotationszustandssummen $Z_{T}$ der $A$ - und $E$-Spezies von $\mathrm{CH}_{3} \mathrm{OH}$ bei verschiedenen Temperaturen

\begin{tabular}{rlcc}
\hline & & $Z_{T}(A)$ & $Z_{T}(E)$ \\
\hline $298 \mathrm{~K}$ & berechnet aus Referenz 175 & 18092 & $18008^{a}$ \\
$298 \mathrm{~K}$ & Referenz 275 & 18644 & $18644^{a}$ \\
$50 \mathrm{~K}$ & berechnet aus Referenz 175 & 746 & 873 \\
$50 \mathrm{~K}$ & Referenz 275 & 746 & $746^{a}$ \\
$50 \mathrm{~K}$ & Referenz 216 & & 873 \\
$35 \mathrm{~K}$ & berechnet aus Referenz 175 & 411 & 512 \\
$35 \mathrm{~K}$ & Referenz 275 & 411 & $408^{a}$ \\
$25 \mathrm{~K}$ & berechnet aus Referenz 175 & 232 & 310 \\
$25 \mathrm{~K}$ & Referenz 275 & 232 & $226^{a}$ \\
$15 \mathrm{~K}$ & berechnet aus Referenz 175 & 99 & 144 \\
$15 \mathrm{~K}$ & Referenz 275 & 99 & $85^{a}$ \\
\hline
\end{tabular}

$\overline{{ }^{a}}$ Zustandssumme berechnet relativ zur Nullpunktsenergie der $A$-Spezies. Mit dem Faktor $e^{\frac{\Delta E}{k T}}$ kann auf die Zustandssumme relativ zur Nullpunktsenergie der $E$-Zustände umgerechnet werden, die um $\Delta E=5.5 \mathrm{~cm}^{-1}$ höher liegt. 


\section{A. Anhang}

Tabelle A.2.: Wellenzahlen und Intensitäten die zur Berechnung der Q-Zweig Spektren von $\mathrm{CH}_{3} \mathrm{OH}$ verwendet wurden. Gegeben sind die Wellenzahlen $\tilde{\nu}$ und Symmetriespezies der Übergänge und der jeweilige Wert von $K$ im Torsionsgrundzustand $\tau=0$, aus dem alle Übergänge erfolgen. Übergänge erfolgen dann unter Änderung der Schwingungsquantenzahl der Torsion $\Delta \tau$ und der Quantenzahl der Projektionsrichtung der Rotation $\Delta K$. Die Intensitäten der Q-Zweige bei Raumtemperatur $I_{\mathrm{RT}}$ wurden aus Referenz 218 entnommen oder im Falle von Werten in eckigen Klammern willkürlich festgelegt. Die Intensitäten der Q-Zweige im Jet $I_{\text {Jet }}$ bei $25 \mathrm{~K}$ wurden mit Formel 5.2 aus $I_{\mathrm{RT}}$ berechnet, wobei die Zustandssummen nicht beachtet wurden, da nur relative Intensitäten betrachtet werden. Außerdem sind in eckigen Klammern die verbotenen reinen Torsionsfundamentalen ohne Änderung von $K$ für $K=0$ gegeben. Aus ihnen ergibt sich durch Mittelung mit doppelter Gewichtung der $\mathrm{E} \leftrightarrow \mathrm{E}$ Übergänge die Wellenzahl der Torsionfundamentale ohne Tunnelaufspaltung bei $231.3 \mathrm{~cm}^{-1}$.

\begin{tabular}{|c|c|c|c|c|c|c|}
\hline$\tilde{\nu} / \mathrm{cm}^{-1}$ & Symmetrie & $K$ & $\Delta \tau$ & $\Delta K$ & $I_{\mathrm{RT}}$ & $I_{\mathrm{Jet}}$ \\
\hline$[199.8$ & $\mathrm{E}$ & 0 & 1 & 0] & & \\
\hline [294.4 & A & 0 & 1 & $0]$ & & \\
\hline 197.5 & A & 1 & 1 & 1 & 2.75 & 2.62 \\
\hline 199.8 & A & 2 & 1 & -1 & 12.57 & 5.10 \\
\hline 205.1 & $\mathrm{E}$ & -1 & 1 & 1 & 3.59 & 4.67 \\
\hline 206.3 & E & -2 & 1 & -1 & 5.69 & 3.51 \\
\hline 209.8 & E & -3 & 1 & 1 & 4.50 & 0.71 \\
\hline 219.3 & $\mathrm{E}$ & 3 & 1 & 1 & 3.90 & 1.04 \\
\hline 226.0 & A & 0 & 1 & 1 & 6.57 & 9.89 \\
\hline 236.5 & A & 4 & 1 & 1 & 4.80 & 0.37 \\
\hline 238.1 & $\mathrm{E}$ & -3 & 1 & -1 & 6.27 & 0.92 \\
\hline 244.7 & $\mathrm{E}$ & 3 & 1 & -1 & 1.45 & 0.36 \\
\hline 249.1 & A & 2 & 1 & 1 & 14.04 & 5.04 \\
\hline 249.6 & $\mathrm{E}$ & -1 & 1 & -1 & 2.75 & 3.21 \\
\hline 263.3 & E & 1 & 1 & 1 & 7.00 & 4.50 \\
\hline 269.4 & $\mathrm{E}$ & -2 & 1 & 1 & 2.27 & 1.21 \\
\hline 274.7 & E & 2 & 1 & 1 & 2.25 & 1.36 \\
\hline 278.2 & $\mathrm{E}$ & 0 & 1 & -1 & 6.23 & 5.21 \\
\hline 284.3 & A & 1 & 1 & -1 & 8.68 & 6.82 \\
\hline 299.7 & A & 3 & 1 & 1 & 3.99 & 0.84 \\
\hline 343.1 & A & 1 & 2 & -1 & 1.33 & 0.96 \\
\hline 351.9 & E & -2 & 2 & 1 & 1.45 & 0.69 \\
\hline 374.2 & $\mathrm{E}$ & 3 & 2 & -1 & 1.18 & 0.25 \\
\hline 392.5 & A & 3 & 2 & 1 & 2.72 & 0.52 \\
\hline 392.8 & E & 1 & 2 & 1 & [0.9] & 0.49 \\
\hline 416.5 & E & 2 & 2 & 1 & [0.9] & 0.46 \\
\hline 431.2 & A & 2 & 2 & 1 & [2.0] & 0.57 \\
\hline 444.4 & E & -1 & 2 & -1 & [0.9] & 0.83 \\
\hline 448.3 & A & 2 & 2 & -1 & [2.0] & 0.57 \\
\hline 474.5 & A & 0 & 2 & 1 & [2.0] & 2.23 \\
\hline 495.7 & E & 1 & 2 & -1 & [0.9] & 0.46 \\
\hline 506.5 & $\mathrm{E}$ & -1 & 2 & 1 & [0.9] & 0.81 \\
\hline 539.9 & $\mathrm{E}$ & 2 & 2 & -1 & [0.9] & 0.43 \\
\hline 546.2 & $\mathrm{E}$ & 0 & 2 & 1 & [0.9] & 0.60 \\
\hline 574.7 & A & 3 & 2 & -1 & [2.0] & 0.34 \\
\hline 599.2 & A & 1 & 2 & 1 & [2.0] & 1.24 \\
\hline 618.0 & $\mathrm{E}$ & -2 & 2 & -1 & [0.9] & 0.37 \\
\hline 655.6 & A & 1 & 3 & 1 & [0.6] & 0.37 \\
\hline
\end{tabular}


Tabelle A.3.: Wellenzahlen und Intensitäten die zur Berechnung der Q-Zweig Spektren von $\mathrm{CD}_{3} \mathrm{OH}$ verwendet wurden in analoger Aufstellung zu Tabelle A.2. Die Intensitäten der Q-Zweige bei Raumtemperatur $I_{\mathrm{RT}}$ wurden aus Referenz 218 entnommen oder im Falle von Werten in eckigen Klammern willkürlich festgelegt. Die Intensitäten der Q-Zweige im Jet $I_{\text {Jet }}$ bei $25 \mathrm{~K}$ wurden mit Formel 5.2 aus $I_{\mathrm{RT}}$ berechnet, wobei die Zustandssummen nicht beachtet wurden, da nur relative Intensitäten betrachtet werden. Außerdem sind in eckigen Klammern die verbotenen reinen Torsionsfundamentalen ohne Änderung von $K$ für $K=0$ gegeben. Aus ihnen ergibt sich durch Mittelung mit doppelter Gewichtung der $\mathrm{E} \leftrightarrow \mathrm{E}$ Übergänge die Wellenzahl der Torsionfundamentale ohne Tunnelaufspaltung bei $220.0 \mathrm{~cm}^{-1}$.

\begin{tabular}{lrrrrrr}
\hline$\tilde{\nu} / \mathrm{cm}^{-1}$ & Symmetrie & $K$ & $\Delta \tau$ & $\Delta K$ & $I_{\mathrm{RT}}$ & $I_{\text {Jet }}$ \\
\hline$[193.7$ & $\mathrm{E}$ & 0 & 1 & $0]$ & & \\
{$[272.6$} & $\mathrm{A}$ & 0 & 1 & $0]$ & & \\
196.1 & $\mathrm{E}$ & -3 & 1 & 1 & 103 & 45.4 \\
199.0 & $\mathrm{E}$ & -1 & 1 & 1 & 64.9 & 95.5 \\
199.7 & $\mathrm{~A}$ & 4 & 1 & 1 & 104.9 & 34.3 \\
200.4 & $\mathrm{E}$ & 4 & 1 & -1 & 82.6 & 18.8 \\
205.1 & $\mathrm{E}$ & -5 & 1 & -1 & 56.8 & 8.5 \\
205.6 & $\mathrm{~A}$ & 5 & 1 & -1 & 86.1 & 8.6 \\
208.7 & $\mathrm{~A}$ & 0 & 1 & 1 & 86.2 & 135.8 \\
219.2 & $\mathrm{E}$ & -1 & 1 & -1 & 56.6 & 78.8 \\
230.3 & $\mathrm{E}$ & 2 & 1 & 1 & 48.9 & 49.7 \\
235.1 & $\mathrm{~A}$ & 5 & 1 & 1 & 111.3 & 10.3 \\
239.0 & $\mathrm{~A}$ & 4 & 1 & -1 & 40.8 & 12.1 \\
240.7 & $\mathrm{E}$ & 4 & 1 & 1 & 96 & 19.7 \\
241.9 & $\mathrm{~A}$ & 3 & 1 & 1 & 56.2 & 34.5 \\
247.0 & $\mathrm{E}$ & -3 & 1 & -1 & 108 & 41.8 \\
250.4 & $\mathrm{E}$ & 3 & 1 & -1 & 41.3 & 21.2 \\
253.6 & $\mathrm{~A}$ & 2 & 1 & 1 & 158.4 & 97.8 \\
253.9 & $\mathrm{E}$ & -4 & 1 & -1 & 33.6 & 10.3 \\
259.6 & $\mathrm{E}$ & -2 & 1 & 1 & 59.6 & 44.6 \\
259.9 & $\mathrm{E}$ & 1 & 1 & 1 & 115.7 & 96.4 \\
264.3 & $\mathrm{E}$ & 0 & 1 & -1 & 106.4 & 101.0 \\
264.8 & $\mathrm{~A}$ & 1 & 1 & -1 & 116.5 & 107.0 \\
327.2 & $\mathrm{~A}$ & 1 & 2 & -1 & 20 & 16.7 \\
328.3 & $\mathrm{E}$ & -2 & 2 & 1 & 24.2 & 16.3 \\
335.1 & $\mathrm{E}$ & 3 & 2 & -1 & 26.5 & 11.9 \\
345.3 & $\mathrm{~A}$ & 4 & 2 & -1 & 31.9 & 8.0 \\
395.2 & $\mathrm{E}$ & -4 & 2 & -1 & 24.3 & 6.2 \\
408.8 & $\mathrm{~A}$ & 3 & 2 & 1 & {$[13]$} & 6.4 \\
423.3 & $\mathrm{E}$ & 2 & 2 & 1 & {$[15]$} & 11.8 \\
438.4 & $\mathrm{~A}$ & 2 & 2 & -1 & {$[13]$} & 6.4 \\
438.4 & $\mathrm{E}$ & -1 & 2 & -1 & {$[15]$} & 15.5 \\
454.1 & $\mathrm{~A}$ & 0 & 2 & 1 & {$[13]$} & 14.6 \\
462.3 & $\mathrm{E}$ & 1 & 2 & -1 & {$[15]$} & 10.0 \\
470.3 & $\mathrm{E}$ & -1 & 2 & 1 & {$[15]$} & 15.2 \\
487.0 & $\mathrm{E}$ & 2 & 2 & -1 & {$[15]$} & 11.3 \\
487.1 & $\mathrm{E}$ & 0 & 2 & 1 & {$[15]$} & 11.3 \\
504.1 & $\mathrm{~A}$ & 3 & 2 & -1 & {$[13]$} & 6.0 \\
512.6 & $\mathrm{E}$ & 1 & 2 & 1 & {$[13]$} & 9.4 \\
538.7 & & 3 & 2 & 1 & {$[15]$} & 5.8 \\
565.2 & $\mathrm{E}$ & & & & &
\end{tabular}




\section{A. Anhang}

Tabelle A.4.: Wellenzahlen und Intensitäten die zur Berechnung der Q-Zweig Spektren von $\mathrm{CH}_{3} \mathrm{OD}$ verwendet wurden in analoger Aufstellung zu Tabelle A.2. Die Intensitäten der Q-Zweige bei Raumtemperatur $I_{\mathrm{RT}}$ wurden aus Referenz 276 entnommen oder im Falle von Werten in eckigen Klammern willkürlich festgelegt. Die Intensitäten der Q-Zweige im Jet $I_{\text {Jet }}$ bei $25 \mathrm{~K}$ wurden mit Formel 5.2 aus $I_{\mathrm{RT}}$ berechnet, wobei die Zustandssummen nicht beachtet wurden, da nur relative Intensitäten betrachtet werden. Außerdem sind in eckigen Klammern die verbotenen reinen Torsionsfundamentalen ohne Änderung von $K$ für $K=0$ gegeben. Aus ihnen ergibt sich durch Mittelung mit doppelter Gewichtung der $\mathrm{E} \leftrightarrow \mathrm{E}$ Übergänge die Wellenzahl der Torsionfundamentale ohne Tunnelaufspaltung bei $189.8 \mathrm{~cm}^{-1}$.

\begin{tabular}{|c|c|c|c|c|c|c|}
\hline$\tilde{\nu} / \mathrm{cm}^{-1}$ & Symmetrie & $K$ & $\Delta \tau$ & $\Delta K$ & $I_{\mathrm{RT}}$ & $I_{\mathrm{Jet}}$ \\
\hline$[177.3$ & $\mathrm{E}$ & 0 & 1 & $0]$ & & \\
\hline$[214.7$ & A & 0 & 1 & 0] & & \\
\hline 196.8 & E & 4 & 1 & 1 & [1.9] & 0.24 \\
\hline 198.7 & E & -2 & 1 & 1 & [1.9] & 1.62 \\
\hline 200.3 & $\mathrm{E}$ & 1 & 1 & 1 & 3.39 & 3.92 \\
\hline 206.7 & A & 5 & 1 & 1 & 2.51 & 0.08 \\
\hline 207.4 & $\mathrm{E}$ & 3 & 1 & 1 & 1.74 & 0.69 \\
\hline 208.3 & E & 0 & 1 & -1 & 3.15 & 4.33 \\
\hline 210.2 & A & 1 & 1 & -1 & 4.93 & 6.10 \\
\hline 212.4 & E & -1 & 1 & -1 & 2.12 & 2.78 \\
\hline 222.2 & $\mathrm{E}$ & -4 & 1 & -1 & 2.04 & 0.22 \\
\hline 225.9 & A & 4 & 1 & 1 & 2.57 & 0.33 \\
\hline 227.3 & E & 2 & 1 & 1 & 2.27 & 1.73 \\
\hline 229.4 & A & 3 & 1 & 1 & 4.65 & 1.55 \\
\hline 285.7 & A & 1 & 2 & -1 & 0.39 & 0.41 \\
\hline 290.6 & E & -3 & 2 & 1 & {$[0.5]$} & 0.14 \\
\hline 291.9 & E & -2 & 2 & 1 & 0.42 & 0.29 \\
\hline 301.5 & $\mathrm{E}$ & 0 & 2 & -1 & {$[0.5]$} & 0.57 \\
\hline 304.7 & $\mathrm{E}$ & 2 & 2 & 1 & {$[0.5]$} & 0.33 \\
\hline 313.8 & A & 3 & 2 & 1 & [0.7] & 0.20 \\
\hline 316.5 & E & -1 & 2 & -1 & 0.57 & 0.61 \\
\hline 322.4 & E & 3 & 2 & -1 & [0.5] & 0.16 \\
\hline 324.6 & A & 2 & 2 & -1 & {$[0.7]$} & 0.40 \\
\hline 339.8 & A & 0 & 2 & 1 & 1.14 & 1.41 \\
\hline 342.4 & E & 1 & 2 & 1 & {$[0.5]$} & 0.44 \\
\hline 365.9 & E & 1 & 2 & -1 & {$[0.5]$} & 0.43 \\
\hline 367.5 & $\mathrm{E}$ & 3 & 2 & 1 & 0.39 & 0.12 \\
\hline 368.9 & $\mathrm{E}$ & -1 & 2 & 1 & 0.43 & 0.43 \\
\hline 371.7 & A & 2 & 2 & 1 & {$[0.7]$} & 0.38 \\
\hline 398.1 & $\mathrm{E}$ & -2 & 2 & -1 & {$[0.5]$} & 0.31 \\
\hline 402.0 & $\mathrm{E}$ & 2 & 2 & -1 & {$[0.5]$} & 0.30 \\
\hline 407.6 & A & 3 & 2 & -1 & [0.7] & 0.18 \\
\hline 412.3 & E & 0 & 2 & 1 & [0.5] & 0.50 \\
\hline 416.7 & $\mathrm{E}$ & -3 & 2 & -1 & [0.5] & 0.12 \\
\hline 431.6 & A & 1 & 2 & 1 & {$[0.7]$} & 0.63 \\
\hline 465.8 & A & 1 & 3 & 1 & {$[0.2]$} & 0.18 \\
\hline 472.6 & E & 0 & 3 & 1 & {$[0.1]$} & 0.1 \\
\hline 517.6 & $\mathrm{E}$ & -1 & 3 & 1 & {$[0.1]$} & 0.09 \\
\hline 547.1 & A & 2 & 3 & 1 & {$[0.2]$} & 0.10 \\
\hline 561.0 & A & 2 & 3 & -1 & {$[0.2]$} & 0.10 \\
\hline 576.2 & A & 0 & 3 & 1 & {$[0.2]$} & 0.21 \\
\hline 637.6 & $\mathrm{E}$ & -1 & 3 & -1 & {$[0.1]$} & 0.09 \\
\hline 646.9 & E & 0 & 3 & -1 & {$[0.1]$} & 0.09 \\
\hline
\end{tabular}


Tabelle A.5.: Wellenzahlen und Intensitäten die zur Berechnung der Q-Zweig Spektren von $\mathrm{CD}_{3} \mathrm{OD}$ verwendet wurden in analoger Aufstellung zu Tabelle A.2. Die Intensitäten der Q-Zweige bei Raumtemperatur $I_{\mathrm{RT}}$ wurden aus Referenz 276 entnommen oder im Falle von Werten in eckigen Klammern willkürlich festgelegt. Die Intensitäten der Q-Zweige im Jet $I_{\text {Jet }}$ bei $25 \mathrm{~K}$ wurden mit Formel 5.2 aus $I_{\mathrm{RT}}$ berechnet, wobei die Zustandssummen nicht beachtet wurden, da nur relative Intensitäten betrachtet werden. Außerdem sind in eckigen Klammern die verbotenen reinen Torsionsfundamentalen ohne Änderung von $K$ für $K=0$ gegeben. Aus ihnen ergibt sich durch Mittelung mit doppelter Gewichtung der $\mathrm{E} \leftrightarrow \mathrm{E}$ Übergänge die Wellenzahl der Torsionfundamentale ohne Tunnelaufspaltung bei $176.8 \mathrm{~cm}^{-1}$.

\begin{tabular}{|c|c|c|c|c|c|c|}
\hline$\tilde{\nu} / \mathrm{cm}^{-1}$ & Symmetrie & $K$ & $\Delta \tau$ & $\Delta K$ & $I_{\mathrm{RT}}$ & $I_{\mathrm{Jet}}$ \\
\hline$[168.4$ & $\mathrm{E}$ & 0 & 1 & $0]$ & & \\
\hline 193.4 & A & 0 & 1 & 0] & & \\
\hline 200.01 & A & 3 & 1 & 1 & 51.5 & 38.5790158 \\
\hline 205.88 & $\mathrm{E}$ & -4 & 1 & -1 & 35.3 & 14.3663461 \\
\hline 208.92 & $\mathrm{E}$ & 5 & 1 & 1 & 32.2 & 6.09138333 \\
\hline 209.06 & A & 6 & 1 & 1 & 38 & 2.86533709 \\
\hline 269.66 & A & 3 & 1 & -1 & 49.9 & 43.6366954 \\
\hline 271.61 & $\mathrm{E}$ & -2 & 2 & 1 & [10] & 9.45452397 \\
\hline 274.34 & A & 2 & 1 & -1 & 71.3 & 83.1589831 \\
\hline 276.67 & E & 3 & 2 & -1 & [10] & 6.33731351 \\
\hline 277.07 & E & 0 & 2 & -1 & [10] & 12.488258 \\
\hline 278.47 & $\mathrm{E}$ & 4 & 2 & -1 & [10] & 3.28248669 \\
\hline 287.7 & $\mathrm{E}$ & 1 & 2 & 1 & [10] & 11.1296509 \\
\hline 288.45 & $\mathrm{E}$ & -4 & 2 & 1 & [10] & 3.41090603 \\
\hline 288.45 & $\mathrm{E}$ & -4 & 2 & -1 & [10] & 3.41090603 \\
\hline 291.52 & A & 3 & 2 & 1 & 10.8 & 6.63379809 \\
\hline 291.91 & A & 1 & 1 & -1 & 67.6 & 97.2444704 \\
\hline 294.72 & $\mathrm{E}$ & -3 & 2 & 1 & [10] & 5.64434483 \\
\hline 295.19 & A & 4 & 1 & -1 & 33.6 & 15.9154525 \\
\hline 298.84 & $\mathrm{E}$ & 2 & 2 & 1 & 10.1 & 9.39282283 \\
\hline 302.22 & A & 2 & 2 & 1 & [11] & 9.27198875 \\
\hline 305.36 & A & 6 & 1 & -1 & 28.1 & 2.62228582 \\
\hline 309.17 & $\mathrm{E}$ & -1 & 2 & -1 & 11.1 & 13.139615 \\
\hline 313.1 & A & 5 & 1 & -1 & 37.1 & 7.67417853 \\
\hline 319.63 & $\mathrm{E}$ & -3 & 2 & -1 & [10] & 5.44829258 \\
\hline 321.7 & A & 0 & 2 & 1 & 14.6 & 18.5183608 \\
\hline 334.09 & $\mathrm{E}$ & 1 & 2 & -1 & [10] & 10.4342281 \\
\hline 335.87 & $\mathrm{E}$ & -1 & 2 & 1 & 9.1 & 10.4071519 \\
\hline 339.24 & $\mathrm{E}$ & 4 & 2 & 1 & [10] & 3.01239272 \\
\hline 351.29 & $\mathrm{E}$ & 2 & 2 & -1 & [10] & 8.69785265 \\
\hline 356.22 & $\mathrm{E}$ & 0 & 2 & 1 & [10] & 11.2201583 \\
\hline 362.05 & A & 4 & 2 & 1 & 8.2 & 2.64937788 \\
\hline 372.79 & A & 1 & 2 & -1 & [11] & 13.0342928 \\
\hline 377.84 & $\mathrm{E}$ & 3 & 2 & 1 & [10] & 5.56944054 \\
\hline 378.94 & A & 1 & 2 & 1 & [11] & 11.3407192 \\
\hline 391.38 & $\mathrm{E}$ & -2 & 2 & -1 & [10] & 8.1365718 \\
\hline 409 & A & 4 & 2 & -1 & [11] & 3.92449449 \\
\hline 418.41 & A & 1 & 3 & 1 & [5] & 4.98936867 \\
\hline 420.22 & A & 2 & 2 & -1 & [11] & 9.12625365 \\
\hline 432.32 & $\mathrm{E}$ & 2 & 3 & -1 & {$[2.5]$} & 2.02704853 \\
\hline 437.25 & $\mathrm{E}$ & 0 & 3 & 1 & {$[2.5]$} & 2.61996494 \\
\hline 458.16 & $\mathrm{E}$ & 1 & 3 & -1 & {$[2.5]$} & 2.34546601 \\
\hline 459.94 & $\mathrm{E}$ & -1 & 3 & 1 & {$[2.5]$} & 2.57351474 \\
\hline 480.2 & A & 3 & 2 & -1 & [11] & 6.1474272 \\
\hline 488.72 & A & 0 & 3 & 1 & [5] & 5.52165805 \\
\hline 518.49 & $\mathrm{E}$ & -1 & 3 & -1 & {$[2.5]$} & 2.4985947 \\
\hline 519.67 & A & 3 & 3 & -1 & [5] & 2.69843648 \\
\hline 535.03 & A & 2 & 3 & 1 & [5] & 3.49919056 \\
\hline 560.48 & $\mathrm{E}$ & 1 & 3 & 1 & {$[2.5]$} & 2.23819117 \\
\hline 580.87 & A & 3 & 3 & 1 & [5] & 2.4689191 \\
\hline 587.07 & $\mathrm{E}$ & 0 & 3 & -1 & {$[2.5]$} & 2.44638148 \\
\hline 587.24 & A & 2 & 3 & -1 & [5] & 3.5875588 \\
\hline
\end{tabular}




\section{A. Anhang}

Tabelle A.6.: Ergebnisse der Frequenzvorsagen verschiedener Rechenmethoden zum $\mathrm{O}-\mathrm{H}$ Streckschwingungsbereich und zum Ferninfrarotbereich von $\mathrm{CH}_{3} \mathrm{OH}$. Gegeben sind unskalierte Wellenzahlen $\tilde{\nu}$ in $\mathrm{cm}^{-1}$ und Intensitäten $I$ in $\frac{\mathrm{km}}{\mathrm{mol}}$, sowie die Verhältnisse von experimenteller Wellenzahl der Monomerschwingungen $\left(3684 \mathrm{~cm}^{-1}\right.$ [Vgl. Tabelle 4.4] bzw. $231.3 \mathrm{~cm}^{-1}$ [Vgl. Tabelle A.2]) und Trimerschwingungen $\left(3469^{36} \mathrm{~cm}^{-1}\right.$ bzw. $\left.610 \mathrm{~cm}^{-1}\right)$ zu den berechneten Werten $\frac{\tilde{\nu}_{\mathrm{exp}}}{\tilde{\nu}_{\mathrm{QM}}}$. Die Mittelwerte $\varnothing$ aus den Verhältnissen von Monomer und Trimer werden in Abbildung 5.3 zur Skalierung der vorhergesagten Wellenzahlen in den jeweiligen spektralen Fenstern genutzt. Von den Dimerschwingungen D zeigen jeweils die der Donoren gröBere Verschiebungen als die der Akzeptoren zum Monomer M. Die anharmonischen Rechnungen wurden bis zu den Trimeren T durchgeführt, die harmonischen bis zu den Tetrameren Q, für welche bei tiefen Wellenzahlen zwischenmolekulare Schwingungen oberhalb von $200 \mathrm{~cm}^{-1}$ vorhergesagt werden.

\begin{tabular}{|c|c|c|c|c|c|c|}
\hline & & $\begin{array}{l}\text { B3LYP-D3 } \\
\text { 6-311+G(2d,p) } \\
\text { harmonisch }\end{array}$ & $\begin{array}{c}\text { B3LYP-D3 } \\
\text { 6-311+G(2d,p) } \\
\text { anharmonisch }\end{array}$ & $\begin{array}{c}\text { MP2 } \\
\text { aug-cc-pVTZ } \\
\text { harmonisch }\end{array}$ & $\begin{array}{c}\text { MP2 } \\
\text { TZVP } \\
\text { harmonisch }\end{array}$ & $\begin{array}{c}\text { MP2 } \\
\text { TZVP } \\
\text { anharmonisch }\end{array}$ \\
\hline \multicolumn{7}{|c|}{$\mathrm{O}-\mathrm{H}$ Streckschwingung } \\
\hline $\bar{M}$ & $\frac{\tilde{\nu}_{\exp }}{\tilde{\nu}_{\mathrm{QM}}}$ & 0.959 & 1.007 & 0.955 & 0.949 & 0.995 \\
\hline $\mathrm{T}$ & $\frac{\tilde{\nu}_{\exp }}{\tilde{\nu}_{\mathrm{QM}}}$ & 0.972 & 1.029 & 0.967 & 0.952 & 1.005 \\
\hline$\varnothing$ & $\frac{\tilde{\nu}_{\exp }}{\tilde{\nu}_{\mathrm{QM}}}$ & 0.966 & 1.018 & 0.961 & 0.951 & 1.000 \\
\hline $\mathrm{D}$ & $\tilde{\nu}(I)$ & $3837(44)$ & $3658(34)$ & $3853(50)$ & $3881(40)$ & $3701(32)$ \\
\hline $\mathrm{D}$ & $\tilde{\nu}(I)$ & $3671(545)$ & 3509 (430) & 3695 (498) & 3737 (415) & 3578 (326) \\
\hline $\mathrm{T}$ & $\tilde{\nu}(I)$ & 3575 (930) & 3376 (899) & $3598(829)$ & 3578 (16) & $3465(755)$ \\
\hline $\mathrm{T}$ & $\tilde{\nu}(I)$ & 3567 (841) & 3371 (761) & 3589 (884) & $3642(760)$ & 3451 (1326) \\
\hline $\mathrm{T}$ & $\tilde{\nu}(I)$ & 3498 (11) & 3308 (6) & 3517 (14) & $3650(754)$ & 3399 (18) \\
\hline Q & $\tilde{\nu}(I)$ & 3419 (218) & & 3449 (224) & & \\
\hline Q & $\tilde{\nu}(I)$ & 3374 (2047) & & 3403 (1937) & & \\
\hline Q & $\tilde{\nu}(I)$ & 3374 (2047) & & 3403 (1937) & & \\
\hline Q & $\tilde{\nu}(I)$ & $3262(0)$ & & $3292(0)$ & & \\
\hline \multicolumn{7}{|c|}{ Ferninfrarot } \\
\hline $\bar{M}$ & $\frac{\nu_{\exp }}{\tilde{\nu}_{\mathrm{QM}}}$ & 0.788 & 0.969 & 0.796 & 0.700 & 0.834 \\
\hline $\mathrm{T}$ & $\frac{\tilde{\nu}_{\exp }}{\tilde{\nu}_{\mathrm{QM}}}$ & 0.842 & 0.925 & 0.840 & 0.819 & 0.949 \\
\hline$\varnothing$ & $\frac{\tilde{\nu}_{\exp }}{\tilde{\nu}_{\mathrm{QM}}}$ & 0.815 & 0.947 & 0.818 & 0.760 & 0.892 \\
\hline $\mathrm{D}$ & $\tilde{\nu}(I)$ & 355 (109) & $285(64)$ & $322(90)$ & 375 (111) & 304 (93) \\
\hline D & $\tilde{\nu}(I)$ & 708 (97) & $595(88)$ & 690 (94) & 725 (137) & 609 (118) \\
\hline $\mathrm{T}$ & $\tilde{\nu}(I)$ & 666 (222) & 573 (219) & 662 (179) & 668 (183) & $570(134)$ \\
\hline $\mathrm{T}$ & $\tilde{\nu}(I)$ & $724(238)$ & $659(187)$ & $727(212)$ & $745(275)$ & $642(111)$ \\
\hline $\mathrm{T}$ & $\tilde{\nu}(I)$ & 904 (17) & $813(9)$ & 884 (17) & 869 (39) & 769 (18) \\
\hline Q & $\tilde{\nu}(I)$ & 275 (39) & & $274(38)$ & & \\
\hline Q & $\tilde{\nu}(I)$ & 275 (39) & & $274(38)$ & & \\
\hline Q & $\tilde{\nu}(I)$ & $281(2)$ & & $283(2)$ & & \\
\hline Q & $\tilde{\nu}(I)$ & 799 (27) & & 795 (28) & & \\
\hline Q & $\tilde{\nu}(I)$ & 910 (127) & & 898 (112) & & \\
\hline Q & $\tilde{\nu}(I)$ & 910 (127) & & 898 (112) & & \\
\hline $\mathrm{Q}$ & $\tilde{\nu}(I)$ & $1003(0)$ & & $992(0)$ & & \\
\hline
\end{tabular}


Tabelle A.7.: Ergebnisse der Frequenzvorsagen verschiedener Rechenmethoden zum Ferninfrarotbereich von $\mathrm{CD}_{3} \mathrm{OH}$. Gegeben sind unskalierte Wellenzahlen $\tilde{\nu}$ in $\mathrm{cm}^{-1}$ und Intensitäten $I$ in $\frac{\mathrm{km}}{\mathrm{mol}}$. In Abbildung 5.4 wurden die berechneten Wellenzahlen mit den Faktoren aus Tabelle A.6 skaliert. Bei den Tetrameren Q liegen im Bereich der $\mathrm{O}-\mathrm{H}$ Libration auch $\mathrm{CD}_{3}$ Biegeschwingungen, teilweise mischen diese beiden Schwingungsarten miteinander in den harmonischen Vorhersagen.

\begin{tabular}{lcccccc}
\hline & & $\begin{array}{c}\text { B3LYP-D3 } \\
\text { 6-311+G(2d,p) } \\
\text { harmonisch }\end{array}$ & $\begin{array}{c}\text { B3LYP-D3 } \\
\text { 6-311+G(2d,p) } \\
\text { anharmonisch }\end{array}$ & $\begin{array}{c}\text { MP2 } \\
\text { aug-cc-pVTZ } \\
\text { harmonisch }\end{array}$ & $\begin{array}{c}\text { MP2 } \\
\text { TZVP } \\
\text { harmonisch }\end{array}$ & $\begin{array}{c}\text { MP2 } \\
\text { TZVP } \\
\text { anharmonisch }\end{array}$ \\
\hline $\mathrm{D}$ & $\tilde{\nu}(I)$ & $346(111)$ & $288(57)$ & $312(93)$ & $363(114)$ & $297(94)$ \\
$\mathrm{D}$ & $\tilde{\nu}(I)$ & $703(88)$ & $602(91)$ & $686(89)$ & $719(125)$ & $611(95)$ \\
$\mathrm{T}$ & $\tilde{\nu}(I)$ & $663(215)$ & $569(221)$ & $659(173)$ & $664(177)$ & $571(129)$ \\
$\mathrm{T}$ & $\tilde{\nu}(I)$ & $720(225)$ & $660(163)$ & $722(201)$ & $738(256)$ & $648(174)$ \\
$\mathrm{T}$ & $\tilde{\nu}(I)$ & $843(9)$ & $801(13)$ & $837(10)$ & $833(29)$ & $759(21)$ \\
$\mathrm{Q}$ & $\tilde{\nu}(I)$ & $266(39)$ & & $266(38)$ & & \\
$\mathrm{Q}$ & $\tilde{\nu}(I)$ & $266(39)$ & & $266(38)$ & \\
$\mathrm{Q}$ & $\tilde{\nu}(I)$ & $274.8(2)$ & & $276(2)$ & \\
$\mathrm{Q}$ & $\tilde{\nu}(I)$ & $768.4(20)$ & & $784(21)$ & \\
$\mathrm{Q}$ & $\tilde{\nu}(I)$ & $871(30)$ & & $870(42)$ & \\
$\mathrm{Q}$ & $\tilde{\nu}(I)$ & $871(30)$ & & $870(42)$ & \\
$\mathrm{Q}$ & $\tilde{\nu}(I)$ & $874(0)$ & & $878(0)$ & & \\
$\mathrm{Q}$ & $\tilde{\nu}(I)$ & $897(7)$ & & $905(7)$ & & \\
$\mathrm{Q}$ & $\tilde{\nu}(I)$ & $907(8)$ & & $914(12)$ & & \\
$\mathrm{Q}$ & $\tilde{\nu}(I)$ & $907(8)$ & & $914(12)$ & & \\
$\mathrm{Q}$ & $\tilde{\nu}(I)$ & $910(0)$ & & $919(0)$ & & \\
$\mathrm{Q}$ & $\tilde{\nu}(I)$ & $923(16)$ & & $929(14)$ & & \\
$\mathrm{Q}$ & $\tilde{\nu}(I)$ & $937(96)$ & & $936(69)$ & & \\
$\mathrm{Q}$ & $\tilde{\nu}(I)$ & $937(96)$ & & $936(69)$ & & \\
\hline
\end{tabular}




\section{A. Anhang}

Tabelle A.8.: Ergebnisse der Frequenzvorsagen verschiedener Rechenmethoden zum Ferninfrarotbereich von $\mathrm{CH}_{3} \mathrm{OD}$ und $\mathrm{CD}_{3} \mathrm{OD}$. Gegeben sind unskalierte Wellenzahlen $\tilde{\nu}$ in $\mathrm{cm}^{-1}$ und Intensitäten $I$ in $\frac{\mathrm{km}}{\mathrm{mol}}$, sowie die Verhältnisse von experimenteller Wellenzahl der Monomerschwingungen $\left(189.8 \mathrm{~cm}^{-1}\right.$ [Vgl. Tabelle A.4]) und Trimerschwingungen $\left(460 \mathrm{~cm}^{-1}\right)$ von $\mathrm{CH}_{3} \mathrm{OD}$ zu den berechneten Werten $\frac{\tilde{\nu}_{\text {exp }}}{\tilde{\nu}_{\mathrm{QM}}}$. Die Mittelwerte $\varnothing$ aus den Verhältnissen von $\mathrm{CH}_{3} \mathrm{OD}$-Monomer und -Trimer werden in Abbildung 5.5 und Abbildung 5.6 zur Skalierung der vorhergesagten Wellenzahlen in den jeweiligen spektralen Fenstern genutzt. Für die Dimere von $\mathrm{CD}_{3} \mathrm{OD}$ ist bei der höchsten Wellenzahl die O-D Knickschschwingung des Akzeptors gegeben.

\begin{tabular}{|c|c|c|c|c|}
\hline & & $\begin{array}{c}\text { B3LYP-D3 } \\
\text { 6-311+G(2d,p) } \\
\text { harmonisch }\end{array}$ & $\begin{array}{c}\text { B3LYP-D3 } \\
\text { 6-311+G(2d,p) } \\
\text { anharmonisch }\end{array}$ & $\begin{array}{c}\text { MP2 } \\
\text { aug-cc-pVTZ } \\
\text { harmonisch }\end{array}$ \\
\hline \multicolumn{5}{|c|}{$\mathrm{CH}_{3} \mathrm{OD}$} \\
\hline$\overline{\mathrm{M}}$ & $\frac{\tilde{\nu}_{\exp }}{\tilde{\nu}_{\mathrm{QM}}}$ & 0.814 & 0.955 & 0.824 \\
\hline $\mathrm{T}$ & $\frac{\widetilde{\nu}_{\exp }}{\tilde{\nu}_{\mathrm{QM}}}$ & 0.874 & 0.943 & 0.872 \\
\hline$\varnothing$ & $\frac{\tilde{\nu}_{\exp }^{\text {Lex }}}{\tilde{\tilde{\nu}}}$ & 0.844 & 0.949 & 0.848 \\
\hline $\mathrm{D}$ & $\tilde{\nu}(I)$ & $279(61)$ & $240(43)$ & $255(50)$ \\
\hline D & $\tilde{\nu}(I)$ & $512(56)$ & $448(50)$ & 499 (54) \\
\hline $\mathrm{T}$ & $\tilde{\nu}(I)$ & $482(120)$ & 424 (119) & 479 (97) \\
\hline $\mathrm{T}$ & $\tilde{\nu}(I)$ & $526(135)$ & 488 (108) & $528(122)$ \\
\hline $\mathrm{T}$ & $\tilde{\nu}(I)$ & $664(9)$ & $608(7)$ & 647 (9) \\
\hline Q & $\tilde{\nu}(I)$ & $268(2)$ & & $268(38)$ \\
\hline Q & $\tilde{\nu}(I)$ & 269 (39) & & $268(38)$ \\
\hline Q & $\tilde{\nu}(I)$ & 269 (39) & & $269(2)$ \\
\hline Q & $\tilde{\nu}(I)$ & 584 (17) & & $581(17)$ \\
\hline Q & $\tilde{\nu}(I)$ & $658(78)$ & & $648(67)$ \\
\hline Q & $\tilde{\nu}(I)$ & $658(78)$ & & $648(67)$ \\
\hline Q & $\tilde{\nu}(I)$ & $745(0)$ & & $729(0)$ \\
\hline \multicolumn{5}{|c|}{$\mathrm{CD}_{3} \mathrm{OD}$} \\
\hline $\bar{D}$ & $\tilde{\nu}(I)$ & $268(62)$ & $233(33)$ & $242(52)$ \\
\hline D & $\tilde{\nu}(I)$ & $508(54)$ & $451(47)$ & $495(52)$ \\
\hline D & $\tilde{\nu}(I)$ & $781(26)$ & $772(22)$ & $783(23)$ \\
\hline $\mathrm{T}$ & $\tilde{\nu}(I)$ & 479 (117) & 420 (119) & 476 (94) \\
\hline $\mathrm{T}$ & $\tilde{\nu}(I)$ & $523(131)$ & $490(88)$ & 524 (117) \\
\hline $\mathrm{T}$ & $\tilde{\nu}(I)$ & $654(9)$ & $600(8)$ & $637(9)$ \\
\hline Q & $\tilde{\nu}(I)$ & $261(38)$ & & $261(37)$ \\
\hline Q & $\tilde{\nu}(I)$ & $261(38)$ & & $261(37)$ \\
\hline Q & $\tilde{\nu}(I)$ & $263(2)$ & & $264(2)$ \\
\hline Q & $\tilde{\nu}(I)$ & $578(15)$ & & $575(15)$ \\
\hline Q & $\tilde{\nu}(I)$ & $654(72)$ & & $645(62)$ \\
\hline Q & $\tilde{\nu}(I)$ & $654(72)$ & & $645(62)$ \\
\hline Q & $\tilde{\nu}(I)$ & $728(0)$ & & $715(0)$ \\
\hline
\end{tabular}


Tabelle A.9.: Ergebnisse der harmonischen Frequenzvorsagen zu tert-Butylalkohol auf B3LYPD3/6-311+G(2d,p) Niveau. Gegeben sind unskalierte Wellenzahlen $\tilde{\nu}$ in $\mathrm{cm}^{-1}$ und Intensitäten $I$ in $\frac{\mathrm{km}}{\mathrm{mol}}$. Für den Ferninfrarotbereich kommt es oft zur Mischung der Torsionsbewegung der Hydroxygruppen mit anderen Intramolekularen Schwingungen, die neben den reinen Librationsschwingungen (fett gedruckt) mit angegeben sind. Außerdem sind die Verhältnisse von experimenteller Wellenzahl der Tetramerschwingungen zu den berechneten Werten $\frac{\tilde{\nu}_{\text {exp }}}{\tilde{\nu}_{\mathrm{QM}}}$ für die intensivsten Banden der O-H Streckschwingung (exp. $3259 \mathrm{~cm}^{-137,223}$ ) bzw. Librationsschwingung (exp. $\mathrm{O}-\mathrm{H} 777 \mathrm{~cm}^{-1},{ }^{13} \mathrm{O}-\mathrm{D} 571 \mathrm{~cm}^{-1}$ ) die in Abbildung 5.7 und Abbildung 5.8 zur Skalierung der vorhergesagten Wellenzahlen in den jeweiligen spektralen Fenstern genutzt werden.

\begin{tabular}{|c|c|c|c|c|}
\hline & & $\mathrm{O}-\mathrm{H}$ Streckschw. & $\mathrm{O}-\mathrm{H}$ Torsion & O-D Torsion \\
\hline $\bar{M}$ & $\tilde{\nu}(I)$ & $3813(14)$ & $213(4)$ & $198(42)$ \\
\hline M & $\tilde{\nu}(I)$ & & $267(31)$ & $222(15)$ \\
\hline M & $\tilde{\nu}(I)$ & & $294(75)$ & \\
\hline $\mathrm{D}$ & $\tilde{\nu}(I)$ & $3633(501)$ & $342(28)$ & $257(33)$ \\
\hline D & $\tilde{\nu}(I)$ & 3806 (20) & $372(52)$ & $533(62)$ \\
\hline D & $\tilde{\nu}(I)$ & & 727 (77) & \\
\hline D & $\tilde{\nu}(I)$ & & $750(22)$ & \\
\hline $\mathrm{T}$ & $\tilde{\nu}(I)$ & 3490 (220) & 634 (185) & $425(21)$ \\
\hline $\mathrm{T}$ & $\tilde{\nu}(I)$ & 3543 (844) & 729 (95) & 441 (19) \\
\hline $\mathrm{T}$ & $\tilde{\nu}(I)$ & 3617 (512) & 755 (13) & $494(68)$ \\
\hline $\mathrm{T}$ & $\tilde{\nu}(I)$ & & 757 (14) & $542(75)$ \\
\hline $\mathrm{T}$ & $\tilde{\nu}(I)$ & & $855(60)$ & $636(33)$ \\
\hline Q & $\tilde{\nu}(I)$ & $3288(0)$ & $752(5)$ & $581(12)$ \\
\hline Q & $\tilde{\nu}(I)$ & 3380 (1665) & $752(7)$ & 647 (65) \\
\hline $\mathrm{Q}$ & $\tilde{\nu}(I)$ & 3380 (1666) & $752(7)$ & $647(65)$ \\
\hline Q & $\tilde{\nu}(I)$ & $3423(282)$ & 777 (19) & $736(0)$ \\
\hline Q & $\tilde{\nu}(I)$ & & $881(62)$ & $758(0)$ \\
\hline $\mathrm{Q}$ & $\tilde{\nu}(I)$ & & $882(62)$ & \\
\hline Q & $\tilde{\nu}(I)$ & & $912(0)$ & \\
\hline Q & $\tilde{\nu}(I)$ & & $930(32)$ & \\
\hline Q & $\tilde{\nu}(I)$ & & $930(31)$ & \\
\hline Q & $\tilde{\nu}(I)$ & & 936 (66) & \\
\hline Q & $\tilde{\nu}(I)$ & & $936(66)$ & \\
\hline $\mathrm{Q}$ & $\tilde{\nu}(I)$ & & $1019(0)$ & \\
\hline Q & $\tilde{\nu}(I)$ & & $1067(0)$ & \\
\hline Q & $\frac{\tilde{\nu}_{\exp }}{\tilde{\nu} \mathrm{OM}}$ & 0.964 & 0.882 & 0.883 \\
\hline
\end{tabular}




\section{A. Anhang}

Tabelle A.10.: Ergebnisse der harmonischen Frequenzvorsagen zu Trifluorethanol auf B3LYP$\mathrm{D} 3 / 6-311+\mathrm{G}(2 \mathrm{~d}, \mathrm{p})$ Niveau. Gegeben sind unskalierte Wellenzahlen $\tilde{\nu}$ in $\mathrm{cm}^{-1}$ und Intensitäten $I$ in $\frac{\mathrm{km}}{\mathrm{mol}}$. Für den Ferninfrarotbereich kommt es oft zur Mischung der Torsionsbewegung der Hydroxygruppen mit anderen Intramolekularen Schwingungen, die neben den reinen Librationsschwingungen (fett gedruckt) mit angegeben sind.

\begin{tabular}{|c|c|c|c|c|}
\hline & & $\mathrm{O}-\mathrm{H}$ Streckschw. & $\mathrm{O}-\mathrm{H}$ Torsion & O-D Torsion \\
\hline $\bar{M}$ & $\tilde{\nu}(I)$ & $3817(50)$ & $312(73)$ & 203 (19) \\
\hline M & $\tilde{\nu}(I)$ & & 377 (61) & $258(52)$ \\
\hline M & $\tilde{\nu}(I)$ & & $412(6)$ & 358 (9) \\
\hline M & $\tilde{\nu}(I)$ & & $523(1)$ & \\
\hline $\mathrm{D}$ & $\tilde{\nu}(I)$ & 3677 (431) & $410(10)$ & 325 (57) \\
\hline $\mathrm{D}$ & $\tilde{\nu}(I)$ & 3795 (120) & $464(163)$ & 366 (35) \\
\hline $\mathrm{D}$ & $\tilde{\nu}(I)$ & & $525(4)$ & $412(14)$ \\
\hline $\mathrm{D}$ & $\tilde{\nu}(I)$ & & $635(64)$ & $463(31)$ \\
\hline $\mathrm{D}$ & $\tilde{\nu}(I)$ & & $656(36)$ & $523(1)$ \\
\hline $\mathrm{D}$ & $\tilde{\nu}(I)$ & & $656(13)$ & \\
\hline $\mathrm{T}$ & $\tilde{\nu}(I)$ & $3520(261)$ & $421(8)$ & $395(73)$ \\
\hline $\mathrm{T}$ & $\tilde{\nu}(I)$ & 3594 (694) & $536(8)$ & $412(13)$ \\
\hline $\mathrm{T}$ & $\tilde{\nu}(I)$ & $3631(713)$ & $539(3)$ & $430(25)$ \\
\hline $\mathrm{T}$ & $\tilde{\nu}(I)$ & & 542 (19) & $446(40)$ \\
\hline $\mathrm{T}$ & $\tilde{\nu}(I)$ & & $568(156)$ & $612(75)$ \\
\hline $\mathrm{T}$ & $\tilde{\nu}(I)$ & & $613(98)$ & 644 (18) \\
\hline $\mathrm{T}$ & $\tilde{\nu}(I)$ & & $652(21)$ & $658(25)$ \\
\hline $\mathrm{T}$ & $\tilde{\nu}(I)$ & & 659 (17) & \\
\hline $\mathrm{T}$ & $\tilde{\nu}(I)$ & & $822(18)$ & \\
\hline $\mathrm{T}$ & $\tilde{\nu}(I)$ & & $823(29)$ & \\
\hline $\mathrm{T}$ & $\tilde{\nu}(I)$ & & $844(140)$ & \\
\hline
\end{tabular}


Tabelle A.11.: Ergebnisse der harmonischen Frequenzvorsagen zu Methyllaktatmonomer und clustern auf B3LYP-D3/6-311+G(2d,p)-Niveau. Neben den Dissoziationsenergien ohne $\left(D_{e}\right)$ und mit Nullpunktskorrektur $\left(D_{0}\right)$ sind auch die unskalierten Wellenzahlen $\tilde{\nu}$ in $\mathrm{cm}^{-1}$ und Intensitäten $I$ in $\frac{\mathrm{km}}{\mathrm{mol}} \operatorname{der} \mathrm{O}-\mathrm{H}$ Streckschwingung und der intensivsten Schwingungen im Ferninfrarotbereich gegeben. In diesen Bereich kommt es oft zur Mischung der Torsionsbewegung der Hydroxylgruppen mit anderen Intramolekularen Schwingungen. Schwingungen mit signifikantem Torsionsanteil sind fett gedruckt. (Tabelle wird auf der nächsten Seite fortgesetzt.)

\begin{tabular}{|c|c|c|c|c|}
\hline & $D_{e}$ & $D_{0}$ & $\mathrm{O}-\mathrm{H}$ Streckschw. & $\mathrm{O}-\mathrm{H}$ Torsion \\
\hline \multirow[t]{2}{*}{ M } & & & 3727 (89) & $354(46)$ \\
\hline & & & & $371(73)$ \\
\hline \multirow[t]{2}{*}{ 81homR } & 40.3 & 34.4 & $3648(85)$ & $552(198)$ \\
\hline & & & $3668(852)$ & $667(27)$ \\
\hline \multirow[t]{2}{*}{ 81hetS } & 39.6 & 33.6 & $3623(263)$ & $546(197)$ \\
\hline & & & $3660(771)$ & \\
\hline \multirow[t]{3}{*}{ 81homS } & 39.6 & 33.3 & $3614(57)$ & $354(34)$ \\
\hline & & & 3634 (1093) & $542(192)$ \\
\hline & & & & $673(34)$ \\
\hline \multirow[t]{3}{*}{ Ahet_3 } & 36.6 & 31.3 & $3702(0)$ & $368(72)$ \\
\hline & & & 3704 (157) & $407(52)$ \\
\hline & & & & 429 (167) \\
\hline \multirow[t]{5}{*}{ Ahom_4 } & 35.8 & 30.2 & 3708 (69) & 353 (49) \\
\hline & & & 3761 (79) & 364 (49) \\
\hline & & & & $415(50)$ \\
\hline & & & & $436(60)$ \\
\hline & & & & $465(71)$ \\
\hline \multirow[t]{4}{*}{ Ahet_6 } & 35.8 & 30.0 & 3685 (374) & $360(57)$ \\
\hline & & & $3732(61)$ & 388 (33) \\
\hline & & & & $391(67)$ \\
\hline & & & & 595 (132) \\
\hline \multirow[t]{4}{*}{ Ahom_1 } & 35.9 & 29.6 & 3609 (556) & $360(37)$ \\
\hline & & & $3689(87)$ & $487(60)$ \\
\hline & & & & $512(49)$ \\
\hline & & & & $701(95)$ \\
\hline \multirow[t]{4}{*}{ Ahet_7 } & 34.7 & 29.6 & $3648(428)$ & $344(50)$ \\
\hline & & & $3735(73)$ & $351(42)$ \\
\hline & & & & $393(51)$ \\
\hline & & & & 610 (109) \\
\hline \multirow[t]{4}{*}{ Ahom_5 } & 33.7 & 29.3 & $3725(22)$ & 353 (77) \\
\hline & & & $3726(114)$ & $357(26)$ \\
\hline & & & & 397 (87) \\
\hline & & & & $401(112)$ \\
\hline \multirow[t]{4}{*}{ Ahet_2 } & 35.0 & 29.3 & $3576(599)$ & $351(53)$ \\
\hline & & & $3700(79)$ & $478(64)$ \\
\hline & & & & $523(73)$ \\
\hline & & & & 705 (59) \\
\hline
\end{tabular}


A. Anhang

Tabelle A.11.: Fortsetzung

\begin{tabular}{|c|c|c|c|c|}
\hline & $D_{e} / \mathrm{kJ} / \mathrm{mol}$ & $D_{0} / \mathrm{kJ} / \mathrm{mol}$ & $\mathrm{O}-\mathrm{H}$ Streckschw. & $\mathrm{O}-\mathrm{H}$ Torsion \\
\hline \multirow[t]{5}{*}{ Ahom_6 } & 35.3 & 28.9 & 3679 (377) & $351(59)$ \\
\hline & & & $3743(56)$ & $419(66)$ \\
\hline & & & & 445 (29) \\
\hline & & & & $595(138)$ \\
\hline & & & & $623(25)$ \\
\hline \multirow{3}{*}{ Ahom_7 } & 33.6 & 28.9 & 3654 (412) & $366(51)$ \\
\hline & & & 3731 (72) & $371(90)$ \\
\hline & & & & $602(102)$ \\
\hline \multirow[t]{4}{*}{ Ahet_8 } & 34.4 & 28.9 & $3720(0)$ & 344 (137) \\
\hline & & & 3721 (135) & $412(67)$ \\
\hline & & & & $463(122)$ \\
\hline & & & & $626(45)$ \\
\hline \multirow[t]{4}{*}{ Ahet_1 } & 34.5 & 27.8 & 3624 (491) & $351(53)$ \\
\hline & & & 3709 (75) & 533 (96) \\
\hline & & & & $615(36)$ \\
\hline & & & & $678(95)$ \\
\hline \multirow{3}{*}{$\mathrm{Tb}$} & 93.2 & 81.7 & 3547 (7) & $663(123)$ \\
\hline & & & $3603(792)$ & $664(122)$ \\
\hline & & & $3605(786)$ & \\
\hline \multirow[t]{4}{*}{ Thet_2 } & 88.4 & 76.4 & 3483 (335) & 649 (114) \\
\hline & & & $3553(728)$ & $687(28)$ \\
\hline & & & 3604 (736) & 723 (105) \\
\hline & & & & 895 (168) \\
\hline \multirow{6}{*}{$\mathrm{Qb}$} & 150.7 & 133.2 & $3518(0)$ & $363(34)$ \\
\hline & & & $3538(1418)$ & 363 (34) \\
\hline & & & $3538(1418)$ & $678(42)$ \\
\hline & & & 3539(434) & 707 (192) \\
\hline & & & & $759(28)$ \\
\hline & & & & 759 (28) \\
\hline \multirow[t]{8}{*}{ Chom } & 144.8 & 127.6 & 3309 (1) & $359(30)$ \\
\hline & & & 3384 (1892) & $431(51)$ \\
\hline & & & 3421 (1902) & $756(69)$ \\
\hline & & & 3446 (158) & 787 (78) \\
\hline & & & & 857 (71) \\
\hline & & & & 930 (71) \\
\hline & & & & 941 (47) \\
\hline & & & & 959 (91) \\
\hline \multirow[t]{8}{*}{ Qhom } & 139.4 & 121.6 & $3522(52)$ & 363 (29) \\
\hline & & & 3534 (1103) & $371(34)$ \\
\hline & & & $3550(377)$ & $673(60)$ \\
\hline & & & $3552(1358)$ & 691 (138) \\
\hline & & & & $705(37)$ \\
\hline & & & & $758(25)$ \\
\hline & & & & $941(47)$ \\
\hline & & & & 997 (27) \\
\hline
\end{tabular}


Tabelle A.12.: Ergebnisse der harmonischen Frequenzvorsagen zu den Monomeren und Dimeren von Ethylenglykol und Propylenglykol auf B3LYP-D3/6-311+G(2d,p)Niveau. Neben den relativen Energieunterschieden ohne $(\Delta E)$ und mit Nullpunktskorrektur $\left(\Delta E_{0}\right)$ sind auch die unskalierten Wellenzahlen $\tilde{\nu}$ in $\mathrm{cm}^{-1}$ und Intensitäten $I$ in $\frac{\mathrm{km}}{\mathrm{mol}}$ der $\mathrm{O}-\mathrm{H}$ Streckschwingung in harmonischer und anharmonischer Vorhersage gegeben. Für letztere wurde zur Optimierung das „VeryTight“"Kriterium verwendet und die Rechnungen mit dem „Superfine“-Gitter vom Programm Gaussian 09 Rev. D01 durchgeführt.

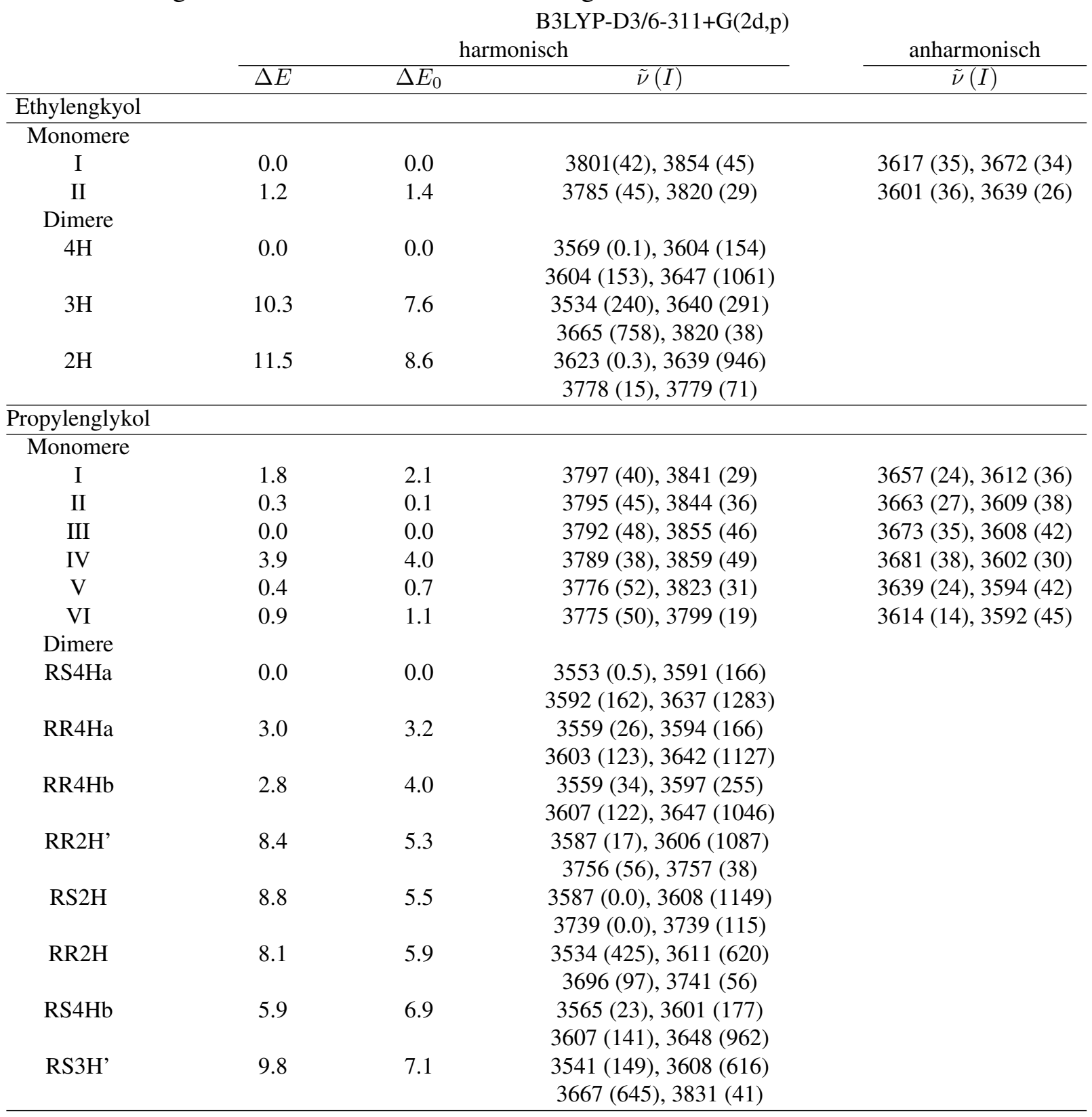


A. Anhang

\section{A.2. Ergänzende Spektren}

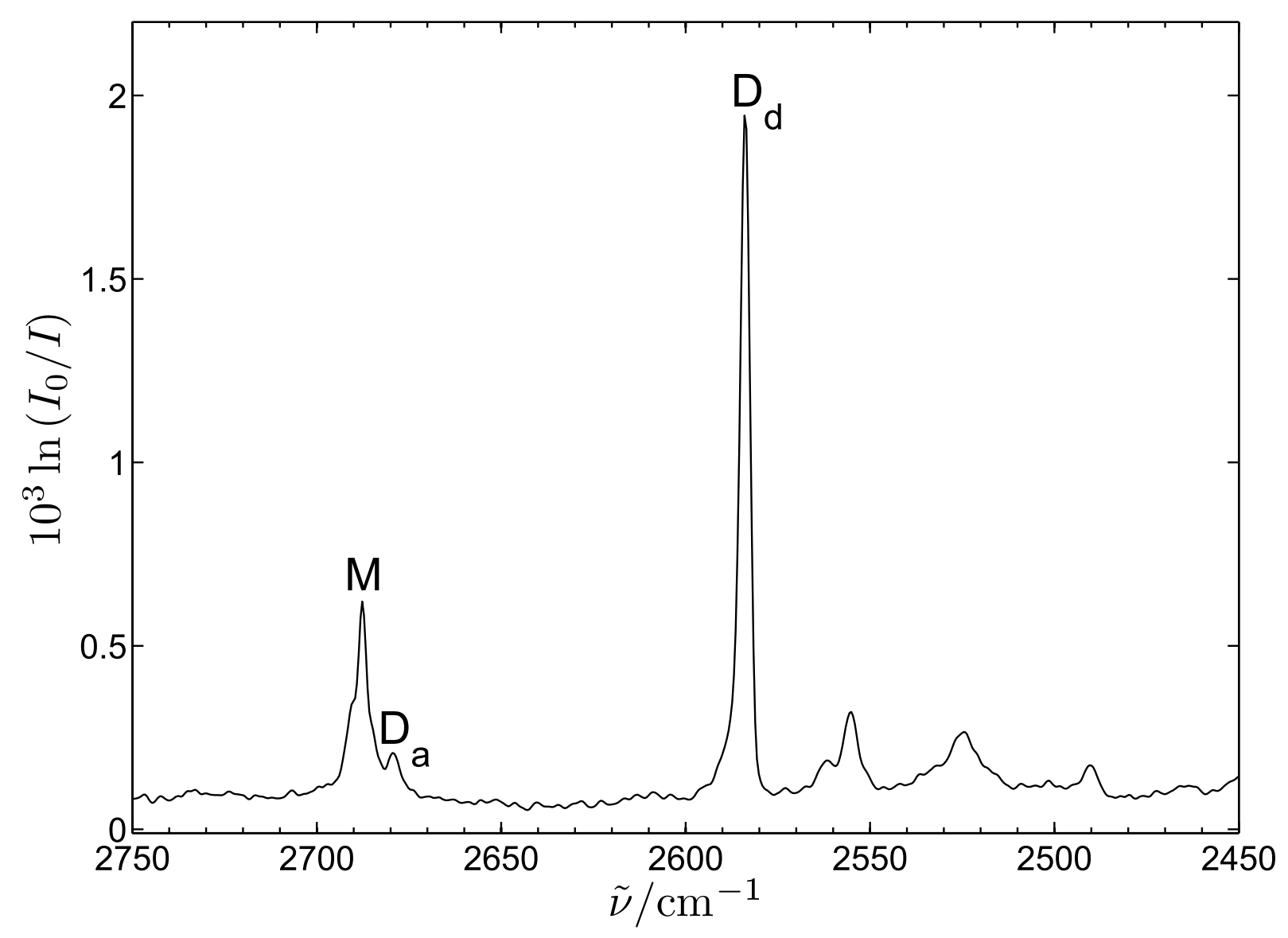

Abbildung A.1.: Das hier gezeigte Jetspektrum von tert-Butylalkohol-OD im Bereich der O-D Streckschwingung wurde von René Wugt Larsen ${ }^{219}$ gemessen. Die Banden können analog zu den Spektren der O-H Spezies zugeordnet werden. Der Monomerübergang liegt bei 2687.7(4) $\mathrm{cm}^{-1}$, der Dimerakzeptor bei 2679.1(6) $\mathrm{cm}^{-1}$ und Dimerdonors bei 2583.9(3). 


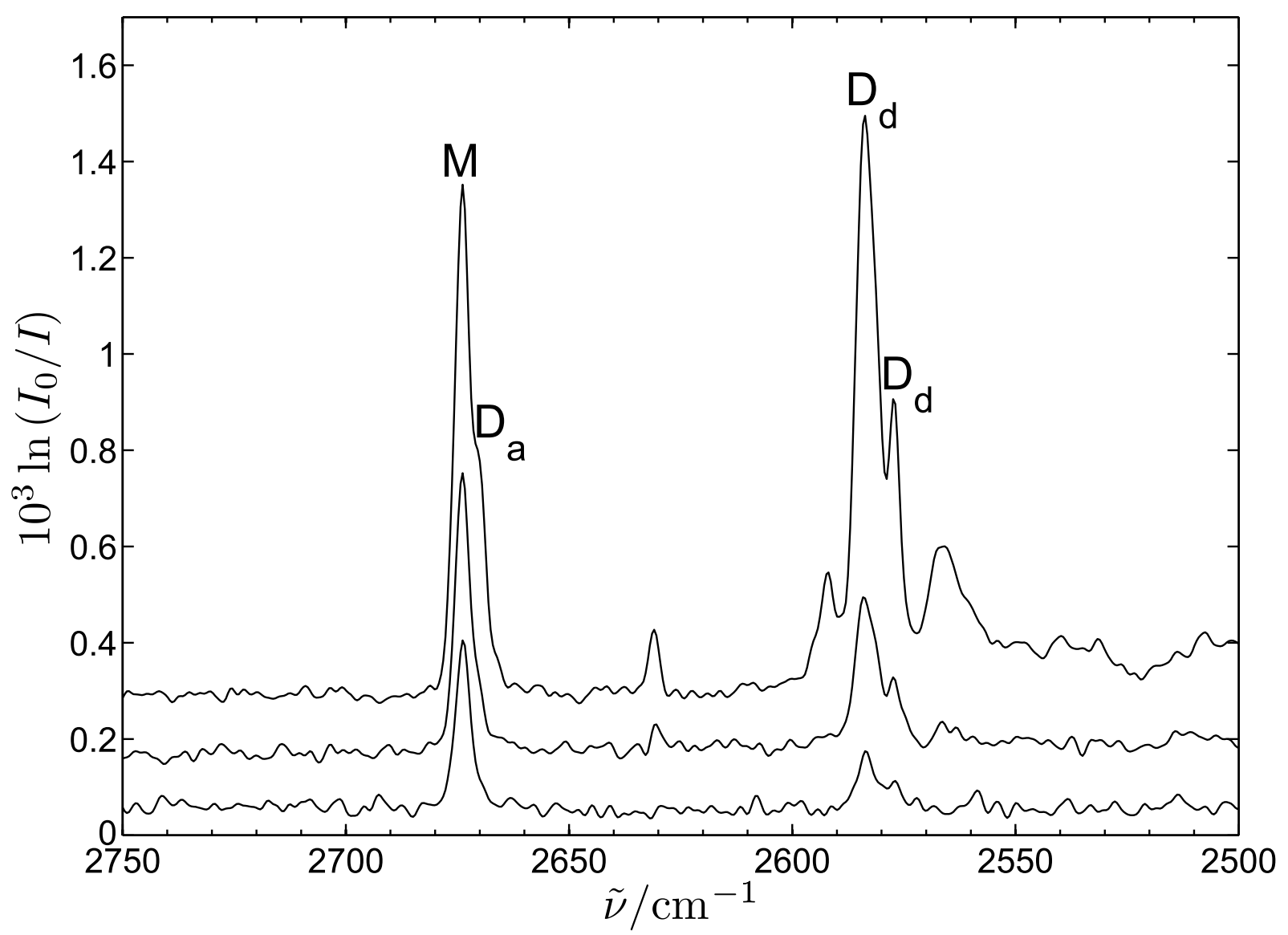

Abbildung A.2.: Das hier gezeigte Jetspektrum von Hexafluorisopropanol-OD im Bereich der O-D Streckschwingung wurde von Tina Scharge im Zuge ihrer Promotion gemessen, ${ }^{43}$ jedoch bisher nicht veröffentlicht. Die Konzentration von Hexafluorisopropanol-OD nimmt in der Abbildung von unten nach oben zu. Die Banden können analog zu den Spektren der $\mathrm{O}-\mathrm{H}$ Spezies und auf Grund ihres Wachstumsverhaltens zugeordnet werden. Der Monomerübergang liegt bei $2673.8(5) \mathrm{cm}^{-1}$. Die Bande des Dimerakzeptors erscheint als Schulter zur Monomerbande bei 2669.9(9) $\mathrm{cm}^{-1}$. Die Dimerdonorschwingung erscheint als Dublett bei $2583.6(2) \mathrm{cm}^{-1}$ und 2577.2(2) $\mathrm{cm}^{-1}$. Der Grund für das Dublett könnte eine Fermiresonanz sein. Unter der Annahme dass die Intensität nur von der O-D Streckschwingung des Dimerdonors kommt, ergibt sich aus dem Intensitätsverhältnis von 3.0(2):1 der beiden Banden zueinander eine entstörte Bandenposition des Dimerdonors von 2582.8(6) $\mathrm{cm}^{-1}$. Die schwache Bande bei $2631 \mathrm{~cm}^{-1}$ könnte von einem weniger stabilen Dimer verursacht werden. Die Banden um den Dimerdonor im Spektrum mit der höchsten Substanzkonzentration stammen von größeren Clustern. 



\section{Literaturverzeichnis}

[1] R. M. Badger, S. H. Bauer, Spectroscopic Studies of the Hydrogen Bond. II. The Shift of the O-H Vibrational Frequency in the Formation of the Hydrogen Bond, The Journal of Chemical Physics 1937, 5, 839-851.

[2] M. Rozenberg, A. Loewenschuss, Y. Marcus, An empirical correlation between stretching vibration redshift and hydrogen bond length, Phys. Chem. Chem. Phys. 2000, 2, 26992702.

[3] E. T. J. Nibbering, J. Dreyer, O. Kühn, J. Bredenbeck, P. Hamm, T. Elsaesser in Analysis and Control of Ultrafast Photoinduced Reactions, Vol. 87 (Eds.: O. Kühn, L. Wöste), Springer Berlin Heidelberg, 2007, pp. 619-687.

[4] J. Joseph, E. D. Jemmis, Red-, Blue-, or No-Shift in Hydrogen Bonds: A Unified Explanation, Journal of the American Chemical Society 2007, 129, 4620-4632.

[5] G. C. Pimentel, A. L. McLellan, The Hydrogen Bond, W. H. Freeman, 1960.

[6] M. A. Suhm, Hydrogen Bond Dynamics in Alcohol Clusters in Advances in Chemical Physics, 142 (Ed.: S. A. Rice), John Wiley \& Sons, Inc., 2008, pp. 1-57.

[7] M. A. Suhm, F. Kollipost, Femtisecond single-mole infrared spectroscopy of molecular clusters, Phys. Chem. Chem. Phys. 2013, 15, 10702-10721.

[8] Y. Matsumoto, J.-i. Iwamoto, K. Honma, Contribution of the $\pi$ electron to the $\mathrm{N}-\mathrm{H} \cdots \mathrm{O}=\mathrm{C}$ hydrogen bond: IR spectroscopic studies of the jet-cooled pyrrole-acetone binary clusters, Phys. Chem. Chem. Phys. 2012, 14, 12938-12947.

[9] F. Kollipost, A. V. Domanskaya, M. A. Suhm, Microscopic Roots of Alcohol-Ketone Demixing: Infrared Spectroscopy of Methanol-Acetone Clusters, J. Phys. Chem. A 2015, $119,2225-2232$.

[10] C. Sándorfy, Hydrogen bonding: How much anharmonicity?, J. Mol. Struct. 2006, 790, 50 -54 .

[11] F. Kollipost, K. Papendorf, Y.-F. Lee, Y.-P. Lee, M. A. Suhm, Alcohol dimers - how much diagonal OH anharmonicity?, Phys. Chem. Chem. Phys. 2014, 16, 15948-15956.

[12] R. Wugt Larsen, M. A. Suhm, Cooperative organic hydrogen bonds: The librational modes of cyclic methanol clusters, J. Chem. Phys. 2006, 125, 154314. 


\section{Literaturverzeichnis}

[13] R. Wugt Larsen, M. A. Suhm, The benefits of alternation and alkylation: Large amplitude hydrogen bond librational modes of alcohol trimers and tetramers, Phys. Chem. Chem. Phys. 2010, 12, 8152-8157.

[14] F. Kollipost, J. Andersen, D. W. Mahler, J. Heimdal, M. Heger, M. Suhm, R.Wugt Larsen, The Effect of Hydrogen Bonding on Torsional Dynamics: A Combined Far-Infrared Jet and Matrix Isolation Study of Methanol Dimer, J. Chem. Phys. 2014, 141, 174314.

[15] F. Kollipost, R. Wugt Larsen, A. V. Domanskaya, M. Nörenberg, M. A. Suhm, Communication: The highest frequency hydrogen bond vibration and an experimental value for the dissociation energy of formic acid dimer, J. Chem. Phys. 2012, 136, 151101.

[16] Z. Xue, Raman spectroscopy of carboxylic acid and water aggregates, Dissertation, Georg-August-Universität Göttingen, 2010.

[17] R. Georges, M. Freytes, D. Hurtmans, I. Kleiner, J. Vander Auwera, M. Herman, Jetcooled and room temperature FTIR spectra of the dimer of formic acid in the gas phase, Chem. Phys. 2004, 305, 187-196.

[18] R. Visinoni, A. Baldacci, P. Stoppa, F. Kollipost, R. Wugt Larsen, High-resolution rovibrational analysis of $\mathrm{CH}_{2}{ }^{81} \mathrm{BrF}$ in the range $920-980 \mathrm{~cm}^{-1}$, Chem. Phys. Lett. 2013, 579, $16-22$.

[19] E. Wilson, J. Decius, P. Cross, Molecular Vibrations: The Theory of Infrared and Raman Vibrational Spectra, Dover Publications, 1980.

[20] J. M. Hollas, High resolution spectroscopy, J. Wiley, 1998.

[21] J. Bloino, V. Barone, A second-order perturbation theory route to vibrational averages and transition properties of molecules: General formulation and application to infrared and vibrational circular dichroism spectroscopies, J. Chem. Phys. 2012, 136, 124108.

[22] J. M. Hollas, Modern Spectroscopy, Wiley, 2004.

[23] F. Jensen, Introduction to Computational Chemistry, Wiley, 2007.

[24] J. Breidung and W. Thiel, Prediction of Vibrational Spectra from Ab Initio Theory in Handbook of High-resolution Spectroscopy (Eds.: M. Quack, F. Merkt), John Wiley \& Sons, Inc., 2011.

[25] J. M. Bowman, The Self-Consistent-Field Approach to Polyatomic Vibrations, Accounts Chem. Res. 1986, 19, 202-208.

[26] J. M. Bowman, X. Huang, N. C. Handy, S. Carter, Vibrational Levels of Methanol Calculated by the Reaction Path Version of MULTIMODE, Using an ab initio, Full-Dimensional Potential , J. Phys. Chem. A 2007, 111, 7317-7321. 
[27] J. M. Bowman, T. Carrington, H.-D. Meyer, Variational quantum approaches for computing vibrational energies of polyatomic molecules, Mol. Phys. 2008, 106, 2145-2182.

[28] H. H. Nielsen, The Vibration-Rotation Energies of Molecules, Rev. Mod. Phys. 1951, 23, 90-136.

[29] V. Barone, Anharmonic vibrational properties by a fully automated second-order perturbative approach, J. Chem. Phys. 2005, 122, 014108.

[30] J. Vázquez, J. F. Stanton, Simple(r) algebraic equation for transition moments of fundamental transitions in vibrational second-order perturbation theory, Mol. Phys. 2006, 104, $377-388$.

[31] V. Barone, J. Bloino, C. A. Guido, F. Lipparini, A fully automated implementation of VPT2 Infrared intensities, Chem. Phys. Lett. 2010, 496, 157 - 161.

[32] P. Schuster, G. Zundel, C. Sandorfy, The Hydrogen Bond, Vol. 2 \& 3, North-Holland, 1976.

[33] E. Arunan, G. R. Desiraju, R. A. Klein, J. Sadlej, S. Scheiner, I. Alkorta, D. C. Clary, R. H. Crabtree, J. J. Dannenberg, P. Hobza, H. G. Kjaergaard, A. C. Legon, B. Mennucci, D. J. Nesbitt, Definition of the hydrogen bond (IUPAC Recommendations 2011), Pure Appl. Chem. 2011, 83, 1637-1641.

[34] A. J. Stone, The Theory of Intermolecular Forces, Oxford University Press, 1996.

[35] P. Hobza, K. Müller-Dethlefs, Non-Covalent Interactions, The Royal Society of Chemistry, 2009, pp. P001-P226.

[36] R. Wugt Larsen, P. Zielke, M. A. Suhm, Hydrogen-bonded OH stretching modes of methanol clusters: A combined IR and Raman isotopomer study, J. Chem. Phys. 2007, 126, 194307.

[37] D. Zimmermann, Th. Häber, H. Schaal, M. A. Suhm, Hydrogen bonded rings, chains and lassos: the case of $t$-butyl alcohol clusters, Mol. Phys. 2001, 99, 413-425.

[38] F. N. Keutsch, R. J. Saykally, Water clusters: Untangling the mysteries of the liquid, one molecule at a time, P. Natl. Acad. Sci. USA 2001, 98, 10533-10540.

[39] S. A. Nizkorodov, M. Ziemkiewicz, D. J. Nesbitt, A. E. W. Knight, Overtone spectroscopy of $\mathrm{H}_{2} \mathrm{O}$ clusters in the $\mathrm{v}_{\mathrm{OH}}=2$ manifold: Infrared-ultraviolet vibrationally mediated dissociation studies, J. Chem. Phys. 2005, 122, 194316.

[40] J. Zischang, M. A. Suhm, The $\mathrm{OH}$ stretching spectrum of warm water clusters, J. Chem. Phys. 2014, 140, 064312.

[41] C. Emmeluth, V. Dyczmons, M. A. Suhm, Tuning the Hydrogen Bond Donor/Acceptor Isomerism in Jet-Cooled Mixed Dimers of Aliphatic Alcohols, J. Phys. Chem. A 2006, 110, 2906-2915. 
Literaturverzeichnis

[42] G. Denisov, J. Mavri, L. Sobczyk in Hydrogen Bonding - New Insights, Vol. 3 (Ed.: S. J. Grabowski), Springer Netherlands, 2006, pp. 377-416.

[43] T. Scharge, D. Luckhaus, M. A. Suhm, Observation and quantification of the hydrogen bond effect on $\mathrm{O}-\mathrm{H}$ overtone intensities in an alcohol dimer, Chem. Phys. 2008, 346, 167 -175 .

[44] F. Huisken, A. Kulcke, C. Laush, J. M. Lisy, Dissociation of small methanol clusters after excitation of the $\mathrm{O}-\mathrm{H}$ stretch vibration at $2.7 \mu$, J. Chem. Phys. 1991, 95, 3924-3929.

[45] M. Fárník, M. Weimann, C. Steinbach, U. Buck, N. Borho, T. B. Adler, M. A. Suhm, Sizeselected methyl lactate clusters: fragmentation and spectroscopic fingerprints of chiral recognition, Phys. Chem. Chem. Phys. 2006, 8, 1148-1158.

[46] T. Scharge, T. Häber, M. A. Suhm, Quantitative chirality synchronization in trifluoroethanol dimers, Phys. Chem. Chem. Phys. 2006, 8, 4664-4667.

[47] T. Scharge, Schwache Bindungen mit starker Wirkung: Konformation und Aggregation von halogenierten Alkoholen, Dissertation, Georg-August-Universität Göttingen, 2007.

[48] T. Di Paolo, C. Bourdéron, C. Sandorfy, Model Calculations on the Influence of Mechanical and Electrical Anharmonicity on Infrared Intensities: Relation to Hydrogen Bonding, Can. J. Chemistry 1972, 50, 3161-3166.

[49] P. Zielke, Ramanstreuung am Überschallstrahl: Wasserstoffbrückendynamik aus neuer Perspektive, Dissertation, Georg-August-Universität Göttingen, 2006.

[50] R. A. Provencal, J. B. Paul, K. Roth, C. Chapo, R. N. Casaes, R. J. Saykally, G. S. Tschumper, H. F. Schaefer III, Infrared cavity ringdown spectroscopy of methanol clusters: Single donor hydrogen bonding, J. Chem. Phys. 1999, 110, 4258-4267.

[51] H.-L. Han, C. Camacho, H. A. Witek, Y.-P. Lee, Infrared absorption of methanol clusters $\left(\mathrm{CH}_{3} \mathrm{OH}\right)_{n}$ with $n=2-6$ recorded with a time-of-flight mass spectrometer using infrared depletion and vacuum-ultraviolet ionization, J. Chem. Phys. 2011, 134, 144309.

[52] H. Günzler, H. M. Heise, IR-Spektroskopie, VCH-Verlag, 1990.

[53] G. Scoles, Atomic and Molecular Beam Methods, Vol. 2, Oxford University Press, 1988.

[54] M. Herman, R. Georges, M. Hepp, D. Hurtmans, High resolution Fourier transform spectroscopy of jet-cooled molecules, Int. Rev. in Phys. Chem. 2000, 19, 277-325.

[55] G. Scoles, Atomic and Molecular Beam Methods, Vol. 1, Oxford University Press, 1988.

[56] M. D. Morse, Supersonic Beam Sources in Experimental Methods in the Physical Sciences, Academic Press, Inc., 1996, pp. 21-47. 
[57] T. Häber, U. Schmitt, M. A. Suhm, FTIR-spectroscopy of molecular clusters in pulsed supersonic slit-jet expansions, Phys. Chem. Chem. Phys. 1999, 1, 5573-5582.

[58] J. Koperski, E. S. Fry, Molecules in the cold environment of a supersonic free-jet beam: from spectroscopy of neutral-neutral interactions to a test of Bell's inequality, J. Phys. B: At. Mol. Opt. 2006, 39, S1125.

[59] T. N. Wassermann, M. A. Suhm, Ethanol Monomers and Dimers Revisited: A Raman Study of Conformational Preferences and Argon Nanocoating Effects, J. Phys. Chem. A 2010, 114, 8223-8233.

[60] T. Häber, U. Schmitt, C. Emmeluth, M. A. Suhm, Ragout-jet FTIR spectroscopy of cluster isomerism and cluster dynamics: from carboxylic acid dimers to $\mathrm{N}_{2} \mathrm{O}$ nanoparticles, Faraday Discuss. 2001, 118, 331-359.

[61] I. Dauster, NH-Schwingungsdynamik in Pyrrolclustern, Diplomarbeit, Georg-AugustUniversität Göttingen, 2005.

[62] N. Borho, Chirale Erkennung in Molekülclustern: Maßgeschneiderte Aggregation von $\alpha-$ Hydroxyestern, Dissertation, Georg-August-Universität Göttingen, 2004.

[63] S. Hesse, Schwache Wechselwirkungen zwischen organischen Molekülen: Strukturelle Vielfalt und ihre schwingungsspektroskopischen Auswirkungen, Dissertation, GeorgAugust-Universität Göttingen, 2009.

[64] M. Albrecht, Biologisch relevante Wasserstoffbrückensysteme im Überschallstrahl: Steuerung der Aggregation durch Substitution, Dissertation, Georg-August-Universität Göttingen, 2009.

[65] J. Lee, Adaptive Aggregation über starke Wasserstoffbrücken in der Gasphase, Dissertation, Georg-August-Universität Göttingen, 2013.

[66] J. Altnöder, Untersuchungen zum Einfluss von London-Dispersionswechselwirkungen auf die Molekülaggregation, Dissertation, Georg-August-Universität Göttingen, 2015.

[67] J. Zischang, Strukturdynamik wasserhaltiger Aggregate in Überschallexpansionen, Dissertation, Georg-August-Universität Göttingen, 2014.

[68] M. J. Frisch, G. W. Trucks, H. B. Schlegel, G. E. Scuseria, M. A. Robb, J. R. Cheeseman, G. Scalmani, V. Barone, B. Mennucci, G. A. Petersson, H. Nakatsuji, M. Caricato, X. Li, H. P. Hratchian, A. F. Izmaylov, J. Bloino, G. Zheng, J. L. Sonnenberg, M. Hada, M. Ehara, K. Toyota, R. Fukuda, J. Hasegawa, M. Ishida, T. Nakajima, Y. Honda, O. Kitao, H. Nakai, T. Vreven, J. A. Montgomery, Jr., J. E. Peralta, F. Ogliaro, M. Bearpark, J. J. Heyd, E. Brothers, K. N. Kudin, V. N. Staroverov, T. Keith, R. Kobayashi, J. Normand, K. Raghavachari, A. Rendell, J. C. Burant, S. S. Iyengar, J. Tomasi, M. Cossi, N. Rega, J. M. 
Literaturverzeichnis

Millam, M. Klene, J. E. Knox, J. B. Cross, V. Bakken, C. Adamo, J. Jaramillo, R. Gomperts, R. E. Stratmann, O. Yazyev, A. J. Austin, R. Cammi, C. Pomelli, J. W. Ochterski, R. L. Martin, K. Morokuma, V. G. Zakrzewski, G. A. Voth, P. Salvador, J. J. Dannenberg, S. Dapprich, A. D. Daniels, O. Farkas, J. B. Foresman, J. V. Ortiz, J. Cioslowski, D. J. Fox, Gaussian09, Revision D.01, Gaussian Inc., Wallingford CT, 2013.

[69] S. Grimme, J. Antony, S. Ehrlich, H. Krieg, A consistent and accurate ab initio parametrization of density functional dispersion correction (DFT-D) for the 94 elements $\mathrm{H}-\mathrm{Pu}, J$. Chem. Phys. 2010, 132, 154104.

[70] S. Grimme, S. Ehrlich, L. Goerigk, Effect of the Damping Function in Dispersion Corrected Density Functional Theory, J. Comput. Chem. 2011, 32, 1456-1465.

[71] D. Falkowski, Warum duften (R)-Limonen und sein Spiegelbild unterschiedlich?, Bachelorarbeit, Georg-August-Universität Göttingen, 2010.

[72] H. Yue, Y. Zhao, X. Ma, J. Gong, Ethylene glycol: properties, synthesis, and applications, Chem. Soc. Rev. 2012, 41, 4218-4244.

[73] K.-M. Marstokk, H. Møllendal, ON THE MICROWAVE SPECTRUM OF ETHYLENE GLYCOL, J. Mol. Struct. 1974, 22, $301-303$.

[74] W. Caminati, G. Corbelli, Conformation of Ethylene Glycol from the Rotational Spectra of the Nontunneling O-monodeuterated Species, J. Mol. Spectroscop. 1981, 90, 572 - 578.

[75] E. Walder, A. Bauder, Hs. H. Günthard, MICROWAVE SPECTRUM AND INTERNAL ROTATIONS OF ETHYLENE GLYCOL. I. GLYCOL-O- $d_{2}$, Chem. Phys. 1980, 51, 223 -239 .

[76] P.-E. Kristiansen, K. Marstokk, H. Møllendal, Microwave Spectrum of $\mathrm{HOCH}_{2} \mathrm{CD}_{2} \mathrm{OH}$, and the Assignment of a Second Hydrogen-Bonded Conformation of Ethylene Glycol, Acta Chem. Scand. 1987, 41, 403-414.

[77] D. Christen, L. Coudert, R. Suenram, F. Lovas, The Rotational/Concerted Torsional Spectrum of the g'Ga Conformer of Ethylene Glycol, J. Mol. Spectroscop. 1995, 172, 57 77.

[78] D. Christen, L. Coudert, J. Larsson, D. Cremer, The Rotational-Torsional Spectrum of the g'Gg Conformer of Ethylene Glycol: Elucidation of an Unusual Tunneling Path, J. Mol. Spectroscop. 2001, 205, 185 - 196.

[79] D. Christen, H. S. P. Müller, The millimeter wave spectrum of $a G g$ ' ethylene glycol: The quest for higher precision, Phys. Chem. Chem. Phys. 2003, 5, 3600-3605.

[80] H. S. P. Müller, D. Christen, Millimeter and submillimeter wave spectroscopic investigations into the rotation-tunneling spectrum of $g G g$ ' ethylene glycol, $\mathrm{HOCH}_{2} \mathrm{CH}_{2} \mathrm{OH}, \mathrm{J}$. Mol. Spectroscop. 2004, 228, 298 - 307. 
[81] J. M. Hollis, F. J. Lovas, P. R. Jewell, L. H. Coudert, INTERSTELLAR ANTIFREEZE: ETHYLENE GLYCOL, Astrophys. J. Lett. 2002, 571, L59.

[82] N. Brouillet, D. Despois, X.-H. Lu, A. Baudry, J. Cernicharo, D. Bockelée-Morvan, J. Crovisier, N. Biver, Antifreeze in the hot core of Orion First detection of ethylene glycol in Orion-KL, Astron. Astrophys. 2015, accepted.

[83] O. Bastiansen, Intra-Molecular Hydrogen Bonds in Ethylene Glycol, Glycerol, and Ethylene Chlorohydrin, Acta Chem. Scand. 1949, 3, 415-421.

[84] M. R. Kazerouni, L. Hedberg, K. Hedberg, Conformational Analysis. 21. Ethane-1,2-diol. An Electron-Diffraction Investigation, Augmented by Rotational Constants and ab Initio Calculations, of the Molecular Structure, Conformational Composition, SQM Vibrational Force Field, and Anti-Gauche Energy Difference with Implications for Internal Hydrogen Bonding, J. Am. Chem. Soc. 1997, 119, 8324-8331.

[85] R. M. Badger, S. H. Bauer, Absorption Spectra of the Vapors of Twelve Alcohols and of Nitric Acid in the Region of the O-H Harmonic Band at $\lambda$ 9500, J. Chem. Phys. 1936, 4, 711-715.

[86] P. Buckley, P. A. Giguère, Infrared studies on rotational isomerism. I. Ethylene glycol, Can. J. Chemistry 1967, 45, 397-407.

[87] D. L. Howard, P. Jørgensen, H. G. Kjaergaard, Weak Intramolecular Interactions in Ethylene Glycol Identified by Vapor Phase OH-Stretching Overtone Spectroscopy, J. Am. Chem. Soc. 2005, 127, 17096-17103.

[88] D. L. Howard, H. G. Kjaergaard, Influence of Intramolecular Hydrogen Bond Strength on OH-Stretching Overtones, J. Phys. Chem. A 2006, 110, 10245-10250.

[89] H. Takeuchi, M. Tasumi, INFRARED-INDUCED CONFORMATIONAL ISOMERIZATION OF ETHYLENE GLYCOL IN A LOW-TEMPERATURE ARGON MATRIX, Chem. Phys. 1983, 77, $21-34$.

[90] T.-K. Ha, H. Frei, R. Meyer, Hs. H. Günthard, Conformation of Ethylene Glycol: Isometric Group, $a b$ initio Study of Internal H Bonding and IR-Matrix Spectra of the Species $\mathrm{CH}_{2} \mathrm{OHCH}_{2} \mathrm{OH}, \mathrm{CD}_{2} \mathrm{OHCD}_{2} \mathrm{OH}$ and $\mathrm{CH}_{2} \mathrm{ODCH}_{2} \mathrm{OD}$, Theor. Chim. Acta 1974, 34, 277292.

[91] H. Frei, T.-K. Ha, R. Meyer, Hs. H. Günthard, ETHYLENE GLYCOL: INFRARED SPECTRA, AB INITIO CALCULATIONS, VIBRATIONAL ANALYSIS AND CONFORMATIONS OF 5 MATRIX ISOLATED ISOTOPIC MODIFICATIONS, Chem. Phys. 1977, 25, $271-298$.

[92] C. G. Park, M. Tasumi, Reinvestigation of Infrared-Induced Conformational Isomerizations of 1,2-Ethanediol in Low-Temperature Ar Matrices and Reverse Reaction in the Dark, J. Phys. Chem. 1991, 95, 2757-2762. 
[93] K. Krishnan, R. S. Krishnan, RAMAN AND INFRARED SPECTRA OF ETHYLENE GLYCOL, P. Indian Acad. of Sci. A 1966, 64, 111-122.

[94] V. Crupi, M. Jannelli, S. Magazu', G. Maisano, D. Majolino, P. Migliardo, D. Sirna, Rayleigh wing and Fourier transform infrared studies of intermolecular and intramolecular hydrogen bonds in liquid ethylene glycol, Mol. Phys. 1995, 84, 645-652.

[95] Y. Chen, Y. Ozaki, M. A. Czarnecki, Molecular structure and hydrogen bonding in pure liquid ethylene glycol and ethylene glycol-water mixtures studied using NIR spectroscopy, Phys. Chem. Chem. Phys. 2013, 15, 18694-18701.

[96] I. Bakó, T. Grósz, G. Pálinkás, M. C. Bellissent-Funel, Ethylene glycol dimers in the liquid phase: A study by x-ray and neutron diffraction, J. Chem. Phys. 2003, 118, 3215-3221.

[97] D. Belashchenko, M. Rodnikova, N. Balabaev, I. Solonina, Investigating Hydrogen Bonds in Liquid Ethylene Glycol Structure by Means of Molecular Dynamics, Russ. J. Phys. Chem. A 2014, 88, 94-102.

[98] A. Kaiser, O. Ismailova, A. Koskela, S. E. Huber, M. Ritter, B. Cosenza, W. Benger, R. Nazmutdinov, M. Probst, Ethylene glycol revisited: Molecular dynamics simulations and visualization of the liquid and its hydrogen-bond network, J. Mol. Liq. 2014, 189, 20 -29 .

[99] H. Matsuura, M. Hiraishi, T. Miyazawa, Raman spectra and energy difference between rotational isomers of ethylene glycol, Spectrochim. Acta A 1972, 28, 2299 - 2304.

[100] C. Murli, N. Lu, Z. Dong, Y. Song, Hydrogen Bonds and Conformations in Ethylene Glycol under Pressure, J. Phys. Chem. B 2012, 116, 12574-12580.

[101] D. Chopra, T. N. Guru Row, E. Arunan, R. A. Klein, Crystalline ethane-1,2-diol does not have intra-molecular hydrogen bonding: Experimental and theoretical charge density studies, J. Mol. Struct. 2010, 964, 126 - 133.

[102] R. M. Kumar, P. Baskar, K. Balamurugan, S. Das, V. Subramanian, On the Perturbation of the H-Bonding Interaction in Ethylene Glycol Clusters upon Hydration, J. Phys. Chem. A 2012, 116, 4239-4247.

[103] Y. Elkadi, L. Adamowicz, Dipole-bound anions of ethylene glycol dimers. Theoretical ab initio study, Chem. Phys. Lett. 1996, 261, $507-514$.

[104] S. Pal, T. K. Kundu, Stability Analysis and Frontier Orbital Study of Different Glycol and Water Complex, ISRN Physical Chemistry 2013, 2013, 753139.

[105] M. Krest'yaninov, A. Titova, A. Zaichikov, Intra- and intermolecular hydrogen bonds in ethylene glycol, monoethanolamine, and ethylenediamine, Russ. J. Phys.Chem. A 2014, $88,2114-2120$. 
[106] F. Lovas, D. Plusquellic, B. H. Pate, J. L. Neill, M. T. Muckle, A. J. Remijan, Microwave spectrum of 1,2-propanediol, J. Mol. Spectroscop. 2009, 257, 82 - 93.

[107] W. Caminati, Conformation and Hydrogen Bond in 1,2-Propanediol, J. Mol. Spectroscop. 1981, 86, $193-201$.

[108] T. J. Lockley, J. I. Hearn, A. K. King, B. J. Howard, Detection and analysis of a new conformational isomer of propan-1,2-diol by Fourier transform microwave spectroscopy, J. Mol. Struct. 2002, 612, 199 - 206.

[109] D. Morantz, M. Waite, Anharmonic effects and intra-molecular hydrogen bonding in alkane-diols, Spectrochim. Acta A 1971, 27, 1133 - 1137.

[110] C. Park, M. Nakata, M. Tasumi, INFRARED-INDUCED CONFORMATIONAL ISOMERIZATION AND REVERSE REACTION IN THE DARK OF 1,2-PROPANEDIOL IN LOW-TEMPERATURE ARGON MATRICES, J. Mol. Struct. 1988, 174, 1 - 4.

[111] R. Friedemann, A. Fengler, S. Naumann, U. Gromann, Theoretical studies on the structure of chiral 1,2-diol systems. Monomers, dimers and monohydrates, J. Mol. Struct. Theochem 1995, 357, $217-223$.

[112] J. Paul, I. Hearn, B. J. Howard, Chiral recognition in a single molecule: a study of homo and heterochiral butan-2,3-diol by Fourier transform microwave spectroscopy, Mol. Phys. 2007, 105, 825-839.

[113] K. Papendorf, Obertöne in Wassersfoffbrücken-gebundenen Alkoholen, Bachelorarbeit, Georg-August-Universität Göttingen, 2012.

[114] J. M. Jasinski, FOURTH OVERTONE SPECTRA OF OH OSCILLATORS IN SIMPLE ALCOHOLS, Chem. Phys. Lett. 1984, 109, 462 - 467.

[115] J. Phillips, J. Orlando, G. Tyndall, V. Vaida, Integrated intensities of OH vibrational overtones in alcohols, Chem. Phys. Lett. 1998, 296, 377 - 383.

[116] K. R. Lange, N. P. Wells, K. S. Plegge, J. A. Phillips, Integrated Intensities of O-H Stretching Bands: Fundamentals and Overtones in Vapor-Phase Alcohols and Acids, J. Phys. Chem. A 2001, 105, 3481-3486.

[117] H. L. Fang, D. M. Meister, R. L. Swofford, Photoacoustic Spectroscopy of Vibrational Overtones in Gas-Phase $\mathrm{CH}_{3} \mathrm{OH}$ and $\mathrm{CH}_{3} \mathrm{OD}$, J. Phys. Chem. 1984, 88, 405-409.

[118] O. V. Boyarkin, L. Lubich, R. D. F. Settle, D. S. Perry, T. R. Rizzo, Intramolecular energy transfer in highly vibrationally excited methanol. I. Ultrafast dynamics, J. Chem. Phys. 1997, 107, 8409-8422.

[119] O. V. Boyarkin, T. R. Rizzo, D. S. Perry, Intramolecular energy transfer in highly vibrationally excited methanol. III. Rotational and torsional analysis, J. Chem. Phys. 1999, 110, 11359-11367. 
Literaturverzeichnis

[120] D. Rueda, O. V. Boyarkin, T. R. Rizzo, I. Mukhopadhyay, D. S. Perry, Torsion-rotation analysis of $\mathrm{OH}$ stretch overtone-torsion combination bands in methanol, J. Chem. Phys. 2002, 116, 91-100.

[121] D. Rueda, O. V. Boyarkin, T. R. Rizzo, A. Chirokolava, D. S. Perry, Vibrational overtone spectroscopy of jet-cooled methanol from 5000 to $14000 \mathrm{~cm}^{-1}$, J. Chem. Phys. 2005, 122, 044314.

[122] H. L. Fang, R. L. Swofford, MOLECULAR CONFORMERS IN GAS-PHASE ETHANOL: A TEMPERATURE STUDY OF VIBRATIONAL OVERTONES, Chem. Phys. Lett. 1984, 105, 5 - 11.

[123] J. D. Weibel, C. F. Jackels, R. L. Swofford, Experimental and ab initio investigation of the O-H overtone vibration in ethanol, J. Chem. Phys. 2002, 117, 4245-4254.

[124] H. L. Fang, D. A. C. Compton, Vibrational overtones of gaseous alcohols, J. Phys. Chem. 1988, 92, 6518-6527.

[125] D. L. Howard, H. G. Kjaergaard, Vapor Phase near Infrared Spectroscopy of the Hydrogen Bonded Methanol-Trimethylamine Complex, J. Phys. Chem. A 2006, 110, 9597-9601.

[126] I. Dauster, C. A. Rice, P. Zielke, M. A. Suhm, N-H $\cdots \pi$ interactions in pyrroles: systematic trends from the vibrational spectroscopy of clusters, Phys. Chem. Chem. Phys. 2008, $10,2827-2835$.

[127] K. von Puttkamer, M. Quack, VIBRATIONAL SPECTRA OF $(\mathrm{HF})_{2},(\mathrm{HF})_{n}$ AND THEIR D-ISOTOPOMERS: MODE SELECTIVE REARRANGEMENTS AND NONSTATISTICAL UNIMOLECULAR DECAY, Chem. Phys. 1989, 139, 31 - 53.

[128] M. A. Suhm, J. T. Farrell, A. McIlroy, D. J. Nesbitt, High resolution $1.3 \mu \mathrm{m}$ overtone spectroscopy of HF dimer in a slit jet: $K_{a}=0 \leftarrow 0$ and $K_{a}=1 \leftarrow 0$ subbands of $\mathrm{v}_{\mathrm{acc}}=2 \leftarrow 0$, J. Chem. Phys. 1992, 97, 5341-5354.

[129] H.-C. Chang, W. Klemperer, High-overtone Spectroscopy and Photodissociation of Hydrogen Fluoride Complexes, Faraday Discuss. 1994, 97, 95-103.

[130] H.-C. Chang, W. Klemperer, The vibrational second overtones of HF dimer: A quartet, $J$. Chem. Phys. 1994, 100, 1-14.

[131] Y. He, H. B. Müller, M. Quack, M. A. Suhm, High Resolution FTIR and Diode Laser Supersonic Jet Spectroscopy of the $N=2$ HF Stretching Polyad in (HF) $)_{2}$ and (HFDF): Hydrogen Bond Switching and Predissociation Dynamics, Z. Phys. Chem. 2007, 221, 1581-1645.

[132] M. Hippler, L. Oeltjen, M. Quack, High-Resolution Continuous-Wave-Diode Laser Cavity Ring-Down Spectroscopy of the Hydrogen Fluoride Dimer in a Pulsed Slit Jet Expansion: Two Components of the $N=2$ Triad near $1.3 \mu \mathrm{m}, J$. Phys. Chem. A 2007, 111, 1265912668. 
[133] J. W. Bevan, B. Martineau, C. Sandorfy, Gas phase observation of the first overtone of the $\mathrm{H}-\mathrm{F}$ stretching fundamental in hydrogen bonded complexes, Can. J. Chemistry 1979, 57, 1341-1349.

[134] B. A. Wofford, S. G. Lieb, J. W. Bevan, Molecular dynamics in hydrogen-bonded interactions: A preliminary experimentally determined harmonic stretching force field for HCN-HF, J. Chem. Phys. 1987, 87, 4478-4486.

[135] J. T. Farrell, Jr., O. Sneh, A. McIlroy, A. E. W. Knight, D. J. Nesbitt, High-resolution infrared overtone spectroscopy of ArHF via Nd:YAG/dye laser difference frequency generation, J. Chem. Phys. 1992, 97, 7967-7978.

[136] P. Block, R. Miller, Infrared-infrared double resonance spectroscopy of Ar-HF. Intermolecular state dependence of the dipole moment and vibrational predissociation in $\nu_{\mathrm{HF}}=2$, Chem. Phys. Lett. 1994, 226, 317 - 324.

[137] H.-C. Chang, F.-M. Tao, W. Klemperer, C. Healey, J. M. Hutson, The Ar-HF intermolecular potential: Overtone spectroscopy and ab initio calculations, J. Chem. Phys. 1993, 99, 9337-9349.

[138] H.-C. Chang, F.-M. Tao, S. N. Tsang, W. Klemperer, Dependence of the Interaction Potentials of Ar-HF and $\mathrm{N}_{2}$-HF on HF Bond Length, J. Chin. Chem. Soc. 1995, 42, 141-148.

[139] C.-C. Chuang, S. N. Tsang, J. G. Hanson, W. Klemperer, H.-C. Chang, Laser-induced fluorescence spectroscopy of $\mathrm{Ar}_{2} \mathrm{HF}$ at $\mathrm{v}_{\mathrm{HF}}=3$ : An examination of three-body forces, $J$. Chem. Phys. 1997, 107, 7041-7056.

[140] C.-C. Chuang, S. N. Tsang, W. Klemperer, H.-C. Chang, Vibrational predissociation of an inert gas cluster containing an active molecule: The $\mathrm{v}_{\mathrm{HF}}=3$ spectrum of $\mathrm{Ar}_{3} \mathrm{HF}$, J. Chem. Phys. 1998, 109, 484-491.

[141] C.-C. Chuang, W. Klemperer, The dependence of intermolecular interactions upon valence coordinate excitation: The $\nu_{\mathrm{HF}}=4$ levels of ArHF, J. Chem. Phys. 2000, 113, 4116-4123.

[142] J. T. Farrell, Jr., O. Sneh, D. J. Nesbitt, High-Resolution Infrared Overtone Spectroscopy of $\mathrm{N}_{2}-\mathrm{HF}$ :Vibrational Red Shifts and Predissociation Rate as a Function of HF Stretching Quanta, J. Phys. Chem. 1994, 98, 6068-6074.

[143] K. M. Beck, M. T. Berry, M. R. Brustein, M. I. Lester, VIBRATIONAL OVERTONE SPECTROSCOPY OF OH-Ar COMPLEXES, Chem. Phys. Lett. 1989, 162, 203 - 210.

[144] M. D. Marshall, B. V. Pond, S. M. Hopman, M. I. Lester, Intermolecular bending levels in an open-shell diatom-diatom complex: Infrared spectroscopy and model calculations of the $\mathrm{OH}-\mathrm{N}_{2}$ complex, J. Chem. Phys. 2001, 114, 7001-7012. 


\section{Literaturverzeichnis}

[145] J. B. Davey, M. E. Greenslade, M. D. Marshall, M. I. Lester, M. D. Wheeler, Infrared spectrum and stability of a $\pi$-type hydrogen-bonded complex between the $\mathrm{OH}$ and $\mathrm{C}_{2} \mathrm{H}_{2}$ reactants, J. Chem. Phys. 2004, 121, 3009-3018.

[146] H. Meyer, E. R. Th. Kerstel, D. Zhuang, G. Scoles, Sub-Doppler rotationally resolved overtone spectroscopy of the HCN dimer, J. Chem. Phys. 1989, 90, 4623-4625.

[147] K. Didriche, C. Lauzin, T. Földes, X. de Ghellinck D’Elseghem Vaernewijck, M. Herman, The FANTASIO+ set-up to investigate jet-cooled molecules: focus on overtone bands of the acetylene dimer, Mol. Phys. 2010, 108, 2155-2163.

[148] K. Didriche, C. Lauzin, T. Földes, D. Golebiowski, M. Herman, C. Leforestier, High resolution overtone spectroscopy of the acetylene van der Waals dimer, $\left({ }^{12} \mathrm{C}_{2} \mathrm{H}_{2}\right)_{2}$, Phys. Chem. Chem. Phys. 2011, 13, 14010-14018.

[149] K. Didriche, C. Lauzin, T. Földes, Observation of the linear $\mathrm{C}_{2} \mathrm{H}_{2}-\mathrm{N}_{2}$ van der Waals complex in the $2 \mathrm{CH}$ range using CW-CRDS, Chem. Phys. Lett. 2012, 530, $31-34$.

[150] D. K. Havey, K. J. Feierabend, K. Takahashi, R. T. Skodje, V. Vaida, Experimental and Theoretical Investigation of Vibrational Overtones of Glycolic Acid and Its Hydrogen Bonding Interactions with Water, J. Phys. Chem. A 2006, 110, 6439-6446.

[151] K. L. Plath, K. Takahashi, R. T. Skodje, V. Vaida, Fundamental and Overtone Vibrational Spectra of Gas-Phase Pyruvic Acid, J. Phys. Chem. A 2009, 113, 7294-7303.

[152] K. Takahashi, K. L. Plath, J. L. Axson, G. C. Nelson, R. T. Skodje, V. Vaida, Dynamics and spectroscopy of vibrational overtone excited glyoxylic acid and 2,2-dihydroxyacetic acid in the gas-phase, J. Chem. Phys. 2010, 132, 094305.

[153] B. J. Miller, J. R. Lane, H. G. Kjaergaard, Intramolecular OH $\cdots \pi$ interactions in alkenols and alkynols, Phys. Chem. Chem. Phys. 2011, 13, 14183-14193.

[154] C. Bourdéron, J.-J. Péron, C. Sandorfy, A Vibrational Overtone Study of Association in Liquid Methanol, J. Phys. Chem. 1972, 76, 869-870.

[155] C. Bourdéron, C. Sandorfy, Association and the assignment of the $\mathrm{OH}$ overtones in hydrogen bonded alcohols, J. Chem. Phys. 1973, 59, 2527-2536.

[156] H. Morita, S. Nagakura, Hydrogen-Bonded O-H and O-D Overtone Bands and Potential Energy Curve of Methanol, J. Mol. Spectroscop. 1974, 49, $401-413$.

[157] A. Burneau, J. Limouzi, Analyse en termes de distributions des absorptions fondamentales et harmoniques de vibrateurs XH en interaction en solution Application aux mesures d'anharmonicité, Mol. Phys. 1977, 33, 399-415.

[158] A. Burneau, J. Perchard, G. Zuppiroli, J. Limouzi, E. Maréchal, Effect of electrical anharmonicity on the profiles of H-bonded $\mathrm{OH}$ and SH stretching bands, Mol. Phys. 1980, 41, 1373-1386. 
[159] S. Singh, D. Schiöberg, W. A. P. Luck, DIPOLE MOMENT DERIVATIVES OF FREE AND HYDROGEN BONDED O-H BOND OF METHANOL, Spectrosc. Lett. 1981, 14, $141-155$.

[160] S. Singh, M. Fritzsche, I. Kümmerle, W. A. P. Luck, H. Y. Zheng, VARIATION OF DIPOLE MOMENT FUNCTION OF O-H BOND OF t-BUTANOL WITH THE NATURE OF SOLVENT AND ITS TEMPERATURE, Spectrosc. Lett. 1985, 18, 283-299.

[161] I. Oref, Solvent Effects in OH Overtone Spectra of Methanol, J. Chem. Soc. Faraday Trans. 1 1986, 82, 1289-1297.

[162] L. England-Kretzer, W.A.P. Luck, BAND ANALYSIS OF $\mathrm{CH}_{3} \mathrm{OH}$ AND $\mathrm{CH}_{3} \mathrm{OD} \mathrm{H-}$ BOND COMPLEXES IN THE FIRST OVERTONE REGION, J. Mol. Struct. 1995, 348, $373-376$.

[163] M. A. Czarnecki, H. Maeda, Y. Ozaki, M. Suzuki, M. Iwahashi, Resolution Enhancement and Band Assignments for the First Overtone of OH Stretching Modes of Butanols by Two-Dimensional Near-Infrared Correlation Spectroscopy. 2. Thermal Dynamics of Hydrogen Bonding in $n$ - and tert-Butyl Alcohol in the Pure Liquid States, J. Phys. Chem. A 1998, 102, 9117-9123.

[164] S. Rai, P. Srivastava, Overtone spectroscopy of butanol, Spectrochim. Acta A 1999, 55, $2793-2800$.

[165] J.-J. Péron, C. Sandorfy, The anharmonicity of the OH stretching vibration of hydrogen bonded methanol in binary systems, J. Chem. Phys. 1976, 65, 3153-3157.

[166] W. Luck, M. Fritzsche, Simultaneous absorption of one quantum by two H-bonded OH groups, J. Mol. Struct. 1993, 295, 47 - 51.

[167] S. Woutersen, Simultaneous photon absorption as a probe of molecular interaction and hydrogen-bond cooperativity in liquids, J. Chem. Phys. 2007, 127, 154517.

[168] L. England, D. Schiöberg, W. A.P. Luck, THE MEANING OF THE VERY DIFFERENT O-H BANDSHAPES IN INFRARED FUNDAMENTAL AND OVERTONE REGIONS OF H-BONDED METHANOL, J. Mol. Struct. 1986, 143, 325 - 328.

[169] L. England-Kretzer, M. Fritzsche, W.A.P. Luck, THE INTENSITY CHANGE OF IR OH BANDS BY H-BONDS, J. Mol. Struct. 1988, 175, 277 - 282.

[170] M. Asselin, G. Bélanger, C. Sandorfy, Anharmonicity and Hydrogen Bonding: Isotopic Substitution, J. Mol. Spectroscop. 1969, 30, 96 - 101.

[171] M. Asselin, C. Sandorfy, Double Excitation in the Infrared Spectra of Self-Associated Alcohols, J. Chem. Phys. 1970, 52, 6130-6134. 
Literaturverzeichnis

[172] J. Perchard, Z. Mielke, Anharmonicity and hydrogen bonding: I. A near-infrared study of methanol trapped in nitrogen and argon matrices, Chem. Phys. 2001, 264, 221 - 234.

[173] V. Hänninen, M. Horn, L. Halonen, Torsional motion and vibrational overtone spectroscopy of methanol, J. Chem. Phys. 1999, 111, 3018-3026.

[174] R. Hunt, W. Shelton, F. A. Flaherty, W. Cook, Torsion-Rotation Energy Levels and the Hindering Potential Barrier for the Excited Vibrational State of the OH-Stretch Fundamental Band $\nu_{1}$ of Methanol, J. Mol. Spectroscop. 1998, 192, 277 - 293.

[175] G. Moruzzi, M. Winnewisser, B. P. Winnewisser, I. Mukhopadhyay, F. Strumia, Microwave, Infrared, and Laser Transitions of Methanol Atlas of Assigned Lines from 0 to $1258 \mathrm{~cm}^{-1}$, Taylor \& Francis, 1995.

[176] J. Perchard, The torsion-vibration spectrum of methanol trapped in neon matrix, Chem. Phys. 2007, 332, 86-94.

[177] J. Perchard, F. Romain, Y. Bouteiller, Determination of vibrational parameters of methanol from matrix-isolation infrared spectroscopy and ab initio calculations. Part 1- Spectral analysis in the domain $11000-200 \mathrm{~cm}^{-1}$, Chem. Phys. 2008, 343, $35-46$.

[178] J. C. Pearson, C. S. Brauer, B. J. Drouin, The asymmetric top-asymmetric frame internal rotation spectrum of ethyl alcohol, J. Mol. Spectroscop. 2008, 251, 394 - 409.

[179] J. P. I. Hearn, R. V. Cobley, B. J. Howard, High-resolution spectroscopy of induced chiral dimers: A study of the dimers of ethanol by Fourier transform microwave spectroscopy, $J$. Chem. Phys. 2005, 123, 134324.

[180] V. Dyczmons, Dimers of Ethanol, J. Phys. Chem. A 2004, 108, 2080-2086.

[181] C. Emmeluth, V. Dyczmons, T. Kinzel, P. Botschwina, M. A. Suhm, M. Yanez, Combined jet relaxation and quantum chemical study of the pairing preferences of ethanol, Phys. Chem. Chem. Phys. 2005, 7, 991-997.

[182] T. N. Wassermann, Umgebungseinflüsse auf die $\mathrm{C}-\mathrm{C}$ - und $\mathrm{C}-\mathrm{O}$-Torsionsdynamik in Molekülen und Molekülaggregaten: Schwingungsspektroskopie bei tiefen Temperaturen, Dissertation, Georg-August-Universität Göttingen, 2009.

[183] J. Durig, R. Larsen, F. Cox, B. J. van der Veken, RAMAN AND FAR-INFRARED SPECTRA OF GASEOUS 1,1,1,3,3,3-HEXAFLUORO-2-PROPANOL, CONFORMATIONAL STABILITY AND BARRIERS TO INTERNAL ROTATION, J. Mol. Struct. 1988, 172, $183-201$.

[184] H. Schaal, T. Häber, M. A. Suhm, Hydrogen Bonding in 2-Propanol. The Effect of Fluorination, J. Phys. Chem. A 2000, 104, 265-274. 
[185] B. Czarnik-Matusewicz, S. Pilorz, D. Bieńko, D. Michalska, Molecular and electronic structures, infrared spectra, and vibrational assignment for $a p$ and $s c$ conformers of hexafluoro- iso-propanol, Vib. Spectrosc. 2008, 47, 44 - 52.

[186] A. J. Barnes, J. Murto, Infra-red Cryogenic Studies. Part 10.-Conformational Isomerism of 1,1,1,3,3,3-hexafluoropropan-2-ol, J. Chem. Soc. Faraday Trans. 2 1972, 68, 1642-1651.

[187] J. Murto, A. Kivinen, R. Viitala, J. Hyömäki, Fluoroalcohols-XX: Infrared and Raman spectra of hexafluoro-2-propanol and its deuterated analogues, Spectrochim. Acta A 1973, $29,1121-1137$.

[188] T. Scharge, C. Cézard, P. Zielke, A. Schütz, C. Emmeluth, M. A. Suhm, A peptide cosolvent under scrutiny: self-aggregation of 2,2,2-trifluoroethanol, Phys. Chem. Chem. Phys. 2007, 9, 4472-4490.

[189] J. R. Lane, H. G. Kjaergaard, XH-stretching overtone transitions calculated using explicitly correlated coupled cluster methods, J. Chem. Phys. 2010, 132, 174304.

[190] A. Bleiber, J. Sauer, The vibrational frequency of the donor OH group in the H-bonded dimers of water, methanol and silanol. Ab initio calculations including anharmonicities, Chem. Phys. Lett. 1995, 238, $243-252$.

[191] C. Cappelli, B. Mennucci, C. O. da Silva, J. Tomasi, Refinements on solvation continuum models: Hydrogen-bond effects on the $\mathrm{OH}$ stretch in liquid water and methanol, J. Chem. Phys. 2000, 112, 5382-5392.

[192] M. Umer, K. Leonhard, Ab Initio Calculations of Thermochemical Properties of Methanol Clusters, J. Phys. Chem. A 2013, 117, 1569-1582.

[193] R. Vianello, B. Kovačević, G. Ambrožič, J. Mavri, Z. B. Maksić, Hydrogen bonding in complex of serine with histidine: computational and spectroscopic study of model compounds, Chem. Phys. Lett. 2004, 400, 117 - 121.

[194] R. L. Rowley, C. M. Tracy, T. A. Pakkanen, Potential energy surfaces for small alcohol dimers. II. Propanol, isopropanol, t-butanol, and sec-butanol, J. Chem. Phys. 2007, 127, 025101 .

[195] K. von Puttkamer, M. Quack, High resolution interferometric FTIR spectroscopy of $(\mathrm{HF})_{2}$ : analysis of a low frequency fundamental near $400 \mathrm{~cm}^{-1}$, Mol. Phys. 1987, 62, 1047-1064.

[196] K. von Puttkamer, M. Quack, M. A. Suhm, INFRARED SPECTRUM AND DYNAMICS OF THE HYDROGEN BONDED DIMER (HF $)_{2}$, Infrared Phys. 1989, 29, 535 - 539.

[197] S. Davis, D. T. Anderson, D. J. Nesbitt, Plucking a hydrogen bond: A near infrared study of all four intermolecular modes in (DF) ${ }_{2}, J$. Chem. Phys. 1996, 105, 6645-6664. 
Literaturverzeichnis

[198] M. Quack, M. A. Suhm, Observation and assignment of the hydrogen bond exchange disrotatory in-plane bending vibration $\nu_{5}$ in $(\mathrm{HF})_{2}$, Chem. Phys. Lett. 1990, 171, $517-$ 524.

[199] E. J. Bohac, R. E. Miller, The trans-bending and F-F stretching vibrations of HF dimer in $\nu_{\mathrm{HF}}=1$ : The influence of intermolecular vibrational excitation on the predissociation dynamics, J. Chem. Phys. 1993, 99, 1537-1544.

[200] D. T. Anderson, S. Davis, D. J. Nesbitt, Probing hydrogen bond potentials via combination band spectroscopy: A near infrared study of the geared bend/van der Waals stretch intermolecular modes in $(\mathrm{HF})_{2}$, J. Chem. Phys. 1996, 104, 6225-6243.

[201] D. T. Anderson, S. Davis, D. J. Nesbitt, Hydrogen bond spectroscopy in the near infrared: Out-of-plane torsion and antigeared bend combination bands in (HF $)_{2}, J$. Chem. Phys. 1996, 105, 4488-4503.

[202] K. D. Kolenbrander, C. E. Dykstra, J. M. Lisy, Torsional vibrational modes of (HF) $)_{3}$ : IRIR double resonance spectroscopy and electrical interaction theory, J. Chem. Phys. 1988, $88,5995-6012$.

[203] P. Asselin, P. Soulard, B. Madebène, M. Goubet, T. R. Huet, R. Georges, O. Pirali, P. Roy, The cyclic ground state structure of the HF trimer revealed by far infrared jet-cooled Fourier transform spectroscopy, Phys. Chem. Chem. Phys. 2014, 16, 4797-4806.

[204] T. A. Blake, S. W. Sharpe, S. S. Xantheas, Rotationally resolved spectroscopy of a librational fundamental band of hydrogen fluoride tetramer, J. Chem. Phys. 2000, 113, 707-718.

[205] M. Cirtog, P. Asselin, P. Soulard, B. Madebène, M. E. Alikhani, Intermolecular vibrations of $\left(\mathrm{CH}_{2}\right)_{2} \mathrm{O}-\mathrm{HF}$ and $-\mathrm{DF}$ hydrogen bonded complexes investigated by Fourier transform infrared spectroscopy and ab initio calculations, Phys. Chem. Chem. Phys. 2010, 12, 12299-12307.

[206] B. McElmurry, L. Rivera-Rivera, K. Scott, Z. Wang, I. Leonov, R. Lucchese, J. Bevan, Studies of low-frequency intermolecular hydrogen-bonded vibrations using a continuous supersonic slit jet mid-infrared quantum cascade laser spectrometer, Chem. Phys. 2012, $409,1-10$.

[207] N. Moazzen-Ahmadi, A. R. W. McKellar, J. W. C. Johns, The Far-Infrared Spectrum of the HCl Dimer, J. Mol. Spectroscop. 1989, 138, 282 - 301.

[208] M. Fárník, S. Davis, D. J. Nesbitt, High-resolution IR studies of hydrogen bonded clusters: Large amplitude dynamics in $(\mathrm{HCl})_{\mathrm{n}}$, Faraday Discuss. 2001, 118, 63-78.

[209] R. Wugt Larsen, F. Hegelund, B. Nelander, Observation and Rovibrational Analysis of the Intermolecular $\mathrm{HCl}$ Libration Band $\nu_{4}^{1}$ in $\mathrm{OC}-\mathrm{HCl}$. Modeling of the Intermolecular Potential Energy Surface, J. Phys. Chem. A 2004, 108, 1524-1530. 
[210] R. Wugt Larsen, F. Hegelund, B. Nelander, Observation and rovibrational analysis of the intermolecular $\mathrm{HCl}$ libration band $\nu_{6}^{1}$ of $\mathrm{HCN}-\mathrm{HCl}, \mathrm{DCN}-\mathrm{HCl}$ and $\mathrm{H}^{13} \mathrm{CN}-\mathrm{HCl}$, Phys. Chem. Chem. Phys. 2005, 7, 1953-1959.

[211] R. Wugt Larsen, F. Hegelund, B. Nelander, Observation and Rovibrational Analysis of the Intermolecular $\mathrm{NH}_{3}$ Libration Band $\nu_{9}^{1}$ of $\mathrm{H}_{3} \mathrm{~N}-\mathrm{HCN}$, J. Phys. Chem. A 2005, 109, 4459-4463.

[212] A. McIntosh, Z. Wang, R. Lucchese, J. Bevan, High frequency wavelength modulation cw slit jet diode laser spectrometer for characterizing ground state intermolecular hydrogen bonded vibrations, Infrared Phys. Techn. 2004, 45, 301 - 314.

[213] Y. Liu, M. Weimann, M. A. Suhm, Extension of panoramic cluster jet spectroscopy into the far infrared: Low frequency modes of methanol and water clusters, Phys. Chem. Chem. Phys. 2004, 6, 3315-3319.

[214] J. Marco, J. Orza, J.-L. Abboud, Fourier transform infrared study of gas phase H-bonding: absorptivities and formation equilibrium constants of fluoroalcohol complexes, Vib. Spectrosc. 1994, 6, $267-283$.

[215] D. Rabli, D. R. Flower, The rotational structure of methanol and its excitation by helium, Mon. Not. R. Astron. Soc. 2010, 403, 2033-2040.

[216] R. M. Lees, ON THE $E_{1}-E_{2}$ LABELING OF ENERGY LEVELS AND THE ANOMALOUS EXCITATION OF INTERSTELLAR METHANOL, Astrophys. J. 1973, 184, 763772 .

[217] M. Hepp, I. Pak, K. Yamada, E. Herbst, G. Winnewisser, Diode Laser Study of the Rotational Redistribution in $\mathrm{CH}_{3} \mathrm{OH}$ during a Supersonic Jet Expansion, J. Mol. Spectroscop. 1994, 166, $66-78$.

[218] V. Stern, C. Belorgeot, J. Kachmarsky, K. D. Möller, Far Infrared Spectrum of the Internal Rotation of $\mathrm{CH}_{3} \mathrm{OH}$ and $\mathrm{CD}_{3} \mathrm{OH}$, J. Mol. Spectroscop. 1977, 67, 244-264.

[219] R. Wugt Larsen, private Mitteilung.

[220] C. Lugez, F. Lovas, J. Hougen, N. Ohashi, Global Analysis of $a-, b-$, and $c$-Type Transitions Involving Tunneling Components of $K=0$ and 1 States of the Methanol Dimer, J. Mol. Spectroscop. 1999, 194, 95 - 112.

[221] E. Cohen, B. Drouin, E. Valenzuela, R. Woods, W. Caminati, A. Maris, S. Melandri, The rotational spectrum of tertiary-butyl alcohol, J. Mol. Spectroscop. 2010, 260, 77 - 83.

[222] J. Korppi-Tommola, Tert-butyl alcohol-matrix i.r. spectra and vibrational assignment, Spectrochim. Acta A 1978, 34, 1077 - 1085. 
Literaturverzeichnis

[223] S. Abraham, Schwingungsspektroskopie nah- und überkritischer Lösungsmittel, Dissertation, Georg-August-Universität Göttingen, 2013.

[224] J. Durig, R. Larsen, TORSIONAL VIBRATIONS AND BARRIERS TO INTERNAL ROTATION FOR ETHANOL AND 2,2,2-TRIFLUOROETHANOL, J. Mol. Struct. 1990, $238,195-222$.

[225] M. L. Senent, Y. G. Smeyers, R. Domínguez-Gómez, M. Villa, Ab initio determination of the far infrared spectra of some isotopic varieties of ethanol, J. Chem. Phys. 2000, 112, 5809-5819.

[226] J. R. Durig, H. Deeb, I. D. Darkhalil, J. J. Klaassen, T. K. Gounev, A. Ganguly, The $r_{0}$ structural parameters, conformational stability, barriers to internal rotation, and vibrational assignments for trans and gauche ethanol, J. Mol. Struct. 2011, 985, 202 - 210.

[227] G. A. Pitsevich, I. Y. Doroshenko, V. Y. Pogorelov, V. Sablinskas, V. Balevicius, Structure and vibrational spectra of gauche- and trans-conformers of ethanol: Nonempirical anharmonic calculations and FTIR spectra in argon matrices, Low. Temp. Phys. 2013, 39, 389-400.

[228] K. Otto, Raman-Spektroskopie kleiner Moleküle und Molekülaggregate im Überschallstrahl nach thermischer Anregung, Dissertation, Georg-August-Universität Göttingen, 2015.

[229] J. Marco, J. Orza, Vapor phase FTIR study of H-bonded complexes of 2,2,2Trifluoroethanol and its deuterated derivatives, J. Mol. Struct. 1992, 267, 33 - 38.

[230] L.-H. Xu, G. T. Fraser, F. J. Lovas, R. D. Suenram, C. W. Gillies, H. E. Warner, J. Z. Gillies, The microwave spectrum and $\mathrm{OH}$ internal rotation dynamics of gauche-2,2,2trifluoroethanol, J. Chem. Phys. 1995, 103, 9541-9548.

[231] M. Senent, A. Perez-Ortega, A. Arroyo, R. Domínguez-Gómez, Theoretical investigation of the torsional spectra of 2,2,2-trifluoroethanol, Chem. Phys. 2001, 266, $19-32$.

[232] M. L. Senent, A. Niño, C. Muñoz-Caro, Y. G. Smeyers, R. Domínguez-Gómez, J. M. Orza, Theoretical Study of the Effect of Hydrogen-Bonding on the Stability and Vibrational Spectrum of Isolated 2,2,2-Trifluoroethanol and Its Molecular Complexes, J. Phys. Chem. A 2002, 106, 10673-10680.

[233] M. B. de Amorim, Torsional Profile of 2,2,2-Trifluoroethanol: A Theoretical Analysis With Basis Set Extrapolation, Int. J. of Quantum Chem. 2008, 108, 2550-2556.

[234] M. Perttilä, Vibrational spectra and normal coordinate analysis of 2,2,2-trichloroethanol and 2,2,2-trifluoroethanol, Spectrochim. Acta A 1979, 35, 585 - 592. 
[235] O. Schrems, W. Luck, FAR-INFRARED STUDIES OF ETHANOLS AND FLUOROETHANOLS TRAPPED IN LOW-TEMPERATURE MATRICES, J. Mol. Struct. 1982, $80,477-484$.

[236] J. Thomas, Y. Xu, Chirality Synchronization in Trifluoroethanol Dimer Revisited: The Missing Heterochiral Dimer, J. Phys. Chem. Lett. 2014, 5, 1850-1855.

[237] N. Borho, Y. Xu, Rotational spectrum of a chiral $\alpha$-hydroxyester: conformation stability and internal rotation barrier heights of methyl lactate, Phys. Chem. Chem. Phys. 2007, 9, $1324-1328$.

[238] N. Borho, M. A. Suhm, Self-organization of lactates in the gas phase, Org. Biomol. Chem. 2003, 1, 4351-4358.

[239] T. B. Adler, N. Borho, M. Reiher, M. A. Suhm, Chirality-Induced Switch in HydrogenBond Topology: Tetrameric Methyl Lactate Clusters in the Gas Phase, Angew. Chem. Int. Edit. 2006, 45, 3440-3445.

[240] P. Zielke, M. A. Suhm, Concerted proton motion in hydrogen-bonded trimers: A spontaneous Raman scattering perspective, Phys. Chem. Chem. Phys. 2006, 8, 2826-2830.

[241] A. Borba, A. Gómez-Zavaglia, L. Lapinski, R. Fausto, Matrix isolation FTIR spectroscopic and theoretical study of methyl lactate, Vib. Spectrosc. 2004, 36, 79 - 88.

[242] T. Adler, Chiralitätsgesteuerte Selbstorganisation in der Gasphase, Diplomarbeit, GeorgAugust-Universität Göttingen, 2005.

[243] Z. Xue, M. A. Suhm, Probing the stiffness of the simplest double hydrogen bond: The symmetric hydrogen bond modes of jet-cooled formic acid dimer, J. Chem. Phys. 2009, $131,054301$.

[244] J. E. Bertie, K. H. Michaelian, The Raman spectra of gaseous formic acid- $h_{2}$ and $-d_{2}, J$. Chem. Phys. 1982, 76, 886-894.

[245] J. E. Bertie, K. H. Michaelian, H. H. Eysel, D. Hager, The Raman-active O-H and O-D stretching vibrations and Raman spectra of gaseous formic acid- $d_{1}$ and -OD, J. Chem. Phys. 1986, 85, 4779-4789.

[246] F. Ito, T. Nakanaga, A jet-cooled infrared spectrum of the formic acid dimer by cavity ring-down spectroscopy, Chem. Phys. Lett. 2000, 318, 571 - 577.

[247] F. Ito, T. Nakanaga, Jet-cooled infrared spectra of the formic acid dimer by cavity ringdown spectroscopy: observation of the $\mathrm{O}-\mathrm{H}$ stretching region, Chem. Phys. 2002, 277, $163-169$.

[248] P. Zielke, M. A. Suhm, Raman jet spectroscopy of formic acid dimers: low frequency vibrational dynamics and beyond, Phys. Chem. Chem. Phys. 2007, 9, 4528-4534. 
Literaturverzeichnis

[249] M. Gantenberg, M. Halupka, W. Sander, Dimerization of Formic Acid - An Example of a "Noncovalent" Reaction Mechanism, Chem. Eur. J. 2000, 6, 1865-1869.

[250] F. Madeja, M. Havenith, K. Nauta, R. E. Miller, J. Chochološová, P. Hobza, Polar isomer of formic acid dimers formed in helium nanodroplets, J. Chem. Phys. 2004, 120, 1055410560 .

[251] R. M. Balabin, Polar (Acyclic) Isomer of Formic Acid Dimer: Gas-Phase Raman Spectroscopy Study and Thermodynamic Parameters, J. Phys. Chem. A 2009, 113, 4910-4918.

[252] K. Marushkevich, M. Siltanen, M. Räsänen, L. Halonen, L. Khriachtchev, Identification of New Dimers of Formic Acid: The Use of a Continuous-Wave Optical Parametric Oscillator in Matrix Isolation Experiments, J. Phys. Chem. Lett. 2011, 2, 695-699.

[253] K. Marushkevich, L. Khriachtchev, M. Räsänen, M. Melavuori, J. Lundell, Dimers of the Higher-Energy Conformer of Formic Acid: Experimental Observation, J. Phys. Chem. A 2012, 116, 2101-2108.

[254] R. C. Millikan, K. S. Pitzer, The Infrared Spectra of Dimeric and Crystalline Formic Acid, J. Am. Chem. Soc. 1958, 80, 3515-3521.

[255] Y. Maréchal, IR spectra of carboxylic acids in the gas phase: A quantitative reinvestigation, J. Chem. Phys. 1987, 87, 6344-6353.

[256] K. Hirota, Y. Nakai, Far Infrared Spectrum of Gaseous Formic Acid, Bull. Chem. Soc. Jpn. 1959, 32, 769-771.

[257] G. L. Carlson, R. E. Witkowski, W. G. Fateley, Far infrared spectra of dimeric and crystalline formic and acetic acids, Spectrochim. Acta 1966, 22, 1117-1123.

[258] D. Clague, A. Novak, Far infrared spectra of homogeneous and heterogeneous dimers of some carboxylic acids, J. Mol. Struct. 1970, 5, 149-152.

[259] K. G. Goroya, Y. Zhu, P. Sun, C. Duan, High resolution jet-cooled infrared absorption spectra of the formic acid dimer: A reinvestigation of the $\mathrm{C}-\mathrm{O}$ stretch region, J. Chem. Phys. 2014, 140, 164311.

[260] F. Ito, Jet-cooled infrared spectra of the formic acid dimer by cavity ring-down spectroscopy: Observation of the $\mathrm{C}-\mathrm{O}$ stretching region and vibrational analysis of the Fermi-triad system, Chem. Phys. Lett. 2007, 447, 202-207.

[261] M. Halupka, W. Sander, A simple method for the matrix isolation of monomeric and dimeric carboxylic acids, Spectrochim. Acta A 1998, 54, 495 - 500.

[262] F. Ito, Infrared spectra of $(\mathrm{HCOOH})_{2}$ and $(\mathrm{DCOOH})_{2}$ in rare gas matrices: A comparative study with gas phase spectra, J. Chem. Phys. 2008, 128, 114310. 
[263] M. Ortlieb, M. Havenith, Proton Transfer in $(\mathrm{HCOOH})_{2}$ : An IR High-Resolution Spectroscopic Study of the Antisymmetric C-O Stretch, J. Phys. Chem. A 2007, 111, 7355-7363.

[264] D. Chamma, O. Henri-Rousseau, IR theory of weak H-bonds: Davydov coupling, Fermi resonances and direct relaxations. I. Basis equations within the linear response theory, Chem. Phys. 1999, 248, $53-70$.

[265] A. K. Roy, A. J. Thakkar, Structures of the formic acid trimer, Chem. Phys. Lett. 2004, $386,162-168$.

[266] F. Ito, Infrared spectra of formic acid clusters in noble gas matrices, J. Mol. Struct. 2015, $1091,203-209$.

[267] L. George, W. Sander, Matrix isolation infrared and ab initio study of the hydrogen bonding between formic acid and water, Spectrochim. Acta A 2004, 60, 3225 - 3232.

[268] K. Marushkevich, L. Khriachtchev, M. Räsänen, Hydrogen Bonding between Formic Acid and Water: Complete Stabilization of the Intrinsically Unstable Conformer, J. Phys. Chem. A 2007, 111, 2040-2042.

[269] D. Priem, T.-K. Ha, A. Bauder, Rotational spectra and structures of three hydrogen-bonded complexes between formic acid and water, J. Chem. Phys. 2000, 113, 169-175.

[270] Ö. Birer, M. Havenith, High-Resolution Infrared Spectroscopy of the Formic Acid Dimer, Annu. Rev. Phys. Chem. 2009, 60, 263-275.

[271] M. Sibert, Protonentransfer im $\mathrm{d}_{1}$-Ameisensäuredimer - Laserspektroskopische Untersuchung eines quantenmechanischen Effektes in der Gasphase, Dissertation, RuhrUniversität zu Bochum, 2004.

[272] F. Madeja, M. Havenith, High resolution spectroscopy of carboxylic acid in the gas phase: Observation of proton transfer in (DCOOH $)_{2}$, J. Chem. Phys. 2002, 117, 7162-7168.

[273] M. Heger, R. A. Mata, M. A. Suhm, Soft hydrogen bonds to alkenes: the methanol-ethene prototype under experimental and theoretical scrutiny, Chem. Sci. 2015, 6, 6738-3745.

[274] M. Heger, M. A. Suhm, R. A. Mata, Communication: Towards the binding energy and vibrational red shift of the simplest organic hydrogen bond: Harmonic constraints for methanol dimer, J. Chem. Phys. 2014, 141, 101105.

[275] G. L. Villanueva, M. A. DiSanti, M. J. Mumma, L.-H. Xu, A QUANTUM BAND MODEL OF THE $\nu_{3}$ FUNDAMENTAL OF METHANOL $\mathrm{CH}_{3} \mathrm{OH}$ AND ITS APPLICATION TO FLUORESCENCE SPECTRA OF COMETS, Astrophys. J. 2012, 747, 37.

[276] V. Stern, N. Goff, J. Kachmarsky, K. D. Möller, Far Infrared Internal Rotation Spectrum of $\mathrm{CH}_{3} \mathrm{OD}$ and $\mathrm{CD}_{3} \mathrm{OD}$, J. Mol. Spectroscop. 1978, 79, 345-362. 



\section{Publikationen}

\section{Publikationen}

Folgende Publikationen sind im Rahmen dieser Arbeit entstanden:

- F. Kollipost, R. Wugt Larsen, A. V. Domanskaya, M. Nörenberg, M. A. Suhm, Communication: The highest frequency hydrogen bond vibration and an experimental value for the dissociation energy of formic acid dimer, J. Chem. Phys. 2012, 136, 151101, DOI: 10.1063/1.4704827 (Ref. 15)

- M. A. Suhm, F. Kollipost, Femtisecond single-mole infrared spectroscopy of molecular clusters, Phys. Chem. Chem. Phys. 2013, 15, 10702-10721, DOI: 10.1039/c3cp51515j (Ref. 7)

- F. Kollipost, K. Papendorf, Y.-F. Lee, Y.-P. Lee, M. A. Suhm, Alcohol dimers - how much diagonal OH anharmonicity?, Phys. Chem. Chem. Phys. 2014, 16, 15948-15956, DOI: 10.1039/c4cp01418a (Ref. 11)

- F. Kollipost, J. Andersen, D. W. Mahler, J. Heimdal, M. Heger, M. Suhm, R.Wugt Larsen, The Effect of Hydrogen Bonding on Torsional Dynamics: A Combined Far-Infrared Jet and Matrix Isolation Study of Methanol Dimer, J. Chem. Phys. 2014, 141, 174314, DOI: 10.1063/1.4900922 (Ref. 14)

- F. Kollipost, A. V. Domanskaya, M. A. Suhm, Microscopic Roots of Alcohol-Ketone Demixing: Infrared Spectroscopy of Methanol-Acetone Clusters, J. Phys. Chem. A 2015, 119, 2225-2232, DOI: 10.1021/jp503999b (Ref. 9)

- R. Visinoni, A. Baldacci, P. Stoppa, F. Kollipost, R. Wugt Larsen, High-resolution rovibrational analysis of $\mathrm{CH}_{2}{ }^{81} \mathrm{BrF}$ in the range $920-980 \mathrm{~cm}^{-1}$, Chem. Phys. Lett. 2013, 579, 16 - 22, DOI: 10.1016/j.cplett.2013.06.002 (Ref. 18) 


\section{Betreute Arbeiten}

Folgende Abschlussarbeiten wurden betreut:

- D. Falkowski, Warum duften $(R)$-Limonen und sein Spiegelbild unterschiedlich?, Bachelorarbeit, Georg-August-Universität Göttingen, 2010 (Ref. 71).

- M. Hendrich, Chiralitätserkennung bei Carvon: Ein einfaches Modellsystem für den Geruchssinn, Bachelorarbeit, 2011.

- S. Nehls, Chiralitätserkennung am Beispiel des Limonens: Einfache Modelle für den Geruchssinn, Bachelorarbeit, 2011.

- K. Papendorf, Obertöne in Wasserstoffbrücken-gebundenen Alkoholen, Bachelorarbeit, Georg-August-Universität Göttingen, 2012 (Ref. 113).

- R. Medel, Infrarotspektroskopie von Alkohol-Alken-Komplexen: konkurrierende schwache Wechselwirkungen, Masterarbeit, Georg-August-Universität Göttingen, 2014. 